\title{
Toxic Chemicals in Cigarette Mainstream Smoke - Hazard and Hoopla*
}

\author{
by \\ Alan Rodgman and Charles R. Green
}

Winston-Salem, NC USA

\section{CONTENTS}

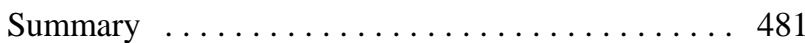

1 Introduction ................. 486

2 Some toxicants in cigarette mainstream smoke - a $21^{\text {st }}$ century list $\ldots \ldots \ldots \ldots \ldots \ldots \ldots 48$

3 Smoking-machine yields in Table 1 for $1 \mathrm{R} 4 \mathrm{~F}$ cigarette .................. 488

4 Obsolete smoke toxicants ............ 488

5 Uncorroborated smoke toxicants . . . . . . . . 495

6 Polychlorodibenzo- $p$-dioxins and polychlorodibenzofurans ... . . . . . . . . . 495

7 Quantitative risk assessments .......... 496

8 Comparison of workplace exposure limits with smoking exposure . . . . . . . . . . . . . . . . . 497

9 Ranking of smoke toxicants by carcinogenic potency database values . . . . . . . . . . . 497

10 Selection of best available carcinogenic potency values for ranking MSS toxicants .......... 499

11 Calculation of incremental cancer lifetime risk for exposure to MSS toxicants . . . . . . . . . 502

12 Qualitative ranking of excess lifetime cancer risk . . . . . . . . . . . . . . . . . . . . . . . . 504

13 Selection of non-cancer health effects toxicity values for ranking MSS toxicants . . . . . . . . 504

14 Calculation of non-cancer risk from exposure to MSS toxicants ... . . . . . . . . . . . . . . . . . . . 504

15 The assertion of the generation of toxicants from additives ............... 506

16 Inhibitors and anticarcinogens in cigarette MSS 510

17 Antimutagens in cigarette MSS . . . . . . . . . . 512

18 The compensation assertion ........... . 514

19 The rise and fall of the major cigarette MSS toxicants: Exception - the tobacco-specific $N$-nitrosamines (TSNAs) . . . . . . . . . . 514

20 The artifactual formation of $N$-nitrosamines . . 515

21 TSNAs in MSS: Direct transfer from tobacco and conflicting data on formation during the smoking process
22 Risk assessments of TSNAs in cigarette MSS . . 516

23 Technologies to control MSS toxicant levels .. 517

24 Cigarette design technologies studied and rejected .................. 518

25 Cigarette design technologies studied and incorporated into commercial products . . . . . 518

26 The US Tobacco Industry criticized: No new cigarette design technology since 1975 . . . . 520

27 TSNAs in flue-cured tobacco: Back to the future $\ldots \ldots \ldots \ldots \ldots \ldots \ldots \ldots \ldots \ldots \ldots$

28 Discussion ...................... 523

29 Conclusions . . . . . . . . . . . . . . . . . . . . . . . 524

30 Acknowledgments ................ 526

31 Glossary .................... 526

References .................... 528

Appendix ...................... 539

\section{SUMMARY}

These are curious times. The Canadian government has passed legislation that requires cigarette manufacturers to routinely test and publish the amounts of 44 toxic substances in cigarette mainstream smoke (MSS). Following in the footsteps of their northern neighbor, various US legislators and regulators are considering modifications to their cigarette testing and reporting programs that will also list toxicants in MSS. Across the Atlantic Ocean, the European Commission has passed a directive that may also follow the North American lead for public disclosure of MSS toxic chemicals for each brand of cigarette sold in the marketplace. United Kingdom authorities have also expressed their intention to follow this mandate.

It is difficult to understand the motivation and value of these existing or potentially forthcoming legislative actions. Although there is nearly total agreement among the world's scientists that cigarette smoking is a health hazard, few are bold enough to say with credibility which smoke chemicals or classes of chemicals are responsible for the adverse

\footnotetext{
* Received: $1^{\text {st }}$ April 2003 - accepted: $8^{\text {th }}$ September 2003

Presented in part at the Invited Speaker Symposium: Cigarette risk and the potential for risk reduction; 2002 CORESTA Congress, New Orleans LA, September 22-27.
} 
effects. Therefore, if the specialists are unable to interpret the smoke toxicant data, how is the general public to use their newfound knowledge?

The posting of smoke chemical toxicant data is also problematic for the Tobacco Industry for several reasons. First, no standard analytical methods exist for most suspected toxicants. Second, the listing of smoke toxicant yields may ignite a $21^{\text {st }}$ Century version of the "tar" wars in the USA during the 1960s; we have already seen evidence of such competition beginning in the US. Third, and most important of all, no one knows whether or not reducing the yield of one or more publicized MSS toxicant will result in a "less hazardous" cigarette.

Assuming that the current situation is approximately as described above, the authors of this paper critically examined the existing lists of MSS toxicants. They discarded chemicals that are no longer relevant, e.g., DDT, $N$-nitrosodiethanolamine, added known smoke constituents that are glaringly absent, e.g., dioxins, and replaced the existing 1950-60s era nonfiltered cigarette MSS yields with those more representative of the present-day marketplace. Data for the Kentucky reference 1R4F cigarette smoked under standardized smoking conditions, i.e., those established by the International Organization for Standardization (ISO) and the Federal Trade Commission (FTC), are used as a surrogate for the modern-day cigarette whenever possible. A list of smoke toxicants and their approximate concentrations in today's cigarettes is nearly useless without an appropriate ranking of their relative toxicity. Unfortunately, the toxicological data for ranking importance are available for fewer than $5 \%$ of the approximately 4800 reported smoke constituents. Although neither of this paper's authors presumes to be a toxicologist, we cite in our discussion several published attempts at ranking smoke toxicants. Specifically, ranking by US Occupational Safety and Health Administration (OSHA) permissible workplace exposure levels, use of US Environmental Protection Agency (EPA) toxicity criteria supplemented with California EPA criteria, and use of the Human Exposure Rodent Potential methodology and database developed by AMES et al. when data are available. There appears to be a wide divergence in the permissible exposures allowable in the workplace and those advocated by environmental regulators. Thus, it is expected that rankings such as those presented herein will ultimately form the basis of MSS toxic chemical prioritization for either attempts at reduction by product developers or development of standardized analytical methods.

This review of MSS toxicants also explores the limitations of toxicological evaluations. The toxicity data used in the above ranking are derived wholly from studies of pure compounds. It is highly improbable that extrapolation of bioassay results determined on an individual compound to that compound when it is a component of a mixture as complex as cigarette MSS is valid. For example, several decades of research involving numerous investigators reported that the benzo $[a]$ pyrene $(\mathrm{B} a \mathrm{P})$ content of cigarette smoke condensate (CSC) accounts for only a few percent of the tumor-bearing animals in the skin-painting bioassay. Subsequently they asserted that the tumorigenic polycyclic aromatic hydrocarbons (PAHs) in CSC could account for no more than 3 to $4 \%$ of the tumorbearing animals. Inclusion of promoters, e.g., phenols, raises the level to about $5 \%$. However, several of the same investigators recently claimed that $\mathrm{B} a \mathrm{P}$ is one of two smoke components responsible for lung cancer in cigarette smokers. While much is written about the hundred or so toxic components in cigarette smoke, little is published about the numerous nontoxic smoke components that have been shown in various bioassays to counteract the effects of the toxic ones. In some cases the inhibiting components are also listed as toxic, e.g., nicotine inhibits the mutagenicity of $N$-nitrosodimethylamine; the promoter phenol inhibits the tumorigenicity of $\mathrm{B} a \mathrm{P}$; the weakly tumorigenic benz $[a]$ anthracene negates the potent tumorigenicity of $\mathrm{B} a \mathrm{P}$. On a one-to-one molar basis, many bicyclic, tricyclic, and tetracyclic nontumorigenic PAHs counteract the tumorigenicity of $\mathrm{B} a \mathrm{P}$ and dibenz $[a, h]$ anthracene.

To further illustrate this murky toxicological situation, the history and current knowledge of the importance of tobacco-specific nitrosamines (TSNAs) to the hazards of smoking is reviewed. In brief, these compounds were discovered in tobacco products and found to transfer to MSS (and sidestream smoke). Toxicological evaluations on the pure compounds demonstrated that they are potent carcinogens. Some public health scientists believed that if the levels of TSNAs could be reduced or lowered in MSS, then this would lead to a "less hazardous" cigarette. Once given this assignment, agronomists discovered that at least for flue-cured tobaccos, the levels of TSNAs can be greatly reduced through the use of indirect heating in the curing barns. This was wonderful news. However, toxicologists soon conducted experiments comparing the toxicity of MSS from flue-cured cigarettes containing high and ultra-low concentrations of TSNAs. It must have been a surprise to these investigators when they could find no significant difference between the toxicities of the two smokes.

Some public health scientists have asserted that the reduction of the per cigarette "tar" delivery below $15 \mathrm{mg} / \mathrm{cig}$ does not reduce the risk from smoking because of the hazard resulting from the higher levels of additives used to maintain consumer acceptability. Although no data in support of this assertion have ever been offered, much data generated during the past decade contradict the assertion. Ingredient addition at the usual level or at levels several times greater than normal does produce some minor changes in the smoke chemistry, but these changes do not result in any adverse biological response as measured in various bioassays to determine mutagenicity, tumorigenicity, etc.

From our review of the literature gathered to prepare this paper, we have come to several conclusions. These include the following:

1 It is possible to prepare a list of the known toxicants in MSS and to prioritize some of them based upon existing biological data. However, for more than $95 \%$ of the known constituents in MSS, there are no biological data.

2 Even if there were biological data for most MSS components, extrapolation of this pure-compound knowledge to the biological properties of a mixture containing them is beyond our scientific ability.

3 At our current state of scientific knowledge, no one will ever be able to legitimately claim the development of a "less hazardous" cigarette based solely on the reduction of known toxic chemicals in MSS. 
4 The approach of reducing "tar" yields of cigarettes appears in retrospect to be the most practical means of producing a "less hazardous" cigarette, because when product developers reduce "tar", both the known and unknown toxicants are reduced.

5 The ranked toxicants in MSS contain both gas-phase and semi-volatile constituents that appear to be important determinants of toxicity. Some of these constituents, e.g., $\mathrm{N}$ nitrosodimethylamine, phenols, are reduced by triacetinplasticized cellulose acetate filters. These filters also reduce "tar". Additionally, it is well known that charcoalcontaining filters have a high efficiency for removing carbonyl compounds from MSS. Development of more consumer-acceptable products that reduce gas-phase toxicants appears to be another route to a "less hazardous" cigarette.

[Beitr. Tabakforsch. Int. 20 (2003) 481-545]

\section{ZUSAMMENFASSUNG}

Heutzutage passieren erstaunliche Dinge. Die kanadische Regierung hat ein Gesetz verabschiedet, dass Zigarettenproduzenten verpflichtet, routinemäßig das mengenmäßige Vorkommen von 44 toxischen Substanzen im Hauptstromrauch (HSR) von Zigaretten zu untersuchen und zu publizieren. Verschiedene gesetzgeberische und regulative Instanzen in den USA treten in die Fußstapfen ihres nördlichen Nachbarn und ziehen Änderungen bei Zigarettentests und in der Publikation der Ergebnisse in Betracht, einschließlich der Veröffentlichung toxischer Substanzen im HSR. Auf der anderen Seite des Atlantiks hat die Europäische Kommission eine Direktive verabschiedet, die auch der nordamerikanischen Vorgabe folgen könnte und Informationen über toxische Substanzen jeder einzelnen im Handel erhältlichen Zigarettenmarke der Öffentlichkeit zugänglich gemacht werden müßten. Auch die verantwortlichen Behörden in Großbritannien haben ihre Absicht bekundet, diesem Mandat zu folgen.

Es ist schwierig, die Motive und den Wert dieser bereits existierenden oder möglichen zukünftigen legislativen Schritte zu verstehen. Obwohl Wissenschaftler auf der ganzen Welt fast ausnahmslos darin übereinstimmen, dass Zigarettenrauchen eine Gefährdung für die Gesundheit darstellt, gibt es nur wenige, die kühn genug sind, mit Glaubwürdigkeit zu sagen, welche chemischen Substanzen oder Substanzklassen für die schädigenden Wirkungen verantwortlich sind. Wenn also Spezialisten nicht dazu in der Lage sind, toxische Rauchdaten zu interpretieren, wie wird dann die Öffentlichkeit ihr neu gefundenes Wissen nutzen? Die Aufstellung der toxikologischen Daten für Rauchbestandteile ist auch für die Tabakindustrie aus mehren Gründen problematisch. Erstens gibt es für die meisten verdächtigen toxischen Substanzen keine standardmäßigen analytischen Nachweismethoden. Zweitens könnte eine Rangliste des Gehalts toxischer Substanzen im Rauch eine Variante des 21. Jahrhunderts der Kondensatkriege der 1960er Jahre in den USA entfachen. Es gibt in den USA schon Anzeichen einer beginnenden derartigen Auseinandersetzung. Drittens und von besonderer Bedeutung ist aber, dass niemand weiß, ob die Verringerung des Gehalts einer oder mehrerer publizierter toxischer Substanzen im
HSR überhaupt zu einer weniger schädlichen Zigarette führt.

Unter der Annahme, dass die gegenwärtige Situation ungefähr so ist wie oben beschrieben, haben die Autoren dieser Arbeit die existierenden Listen toxischer Substanzen im HSR kritisch untersucht. Dabei wurden Substanzen ausgenommen, die nicht länger von Bedeutung sind, wie z.B. DDT, $N$-Nitrosodiethanolamine, bekannte Rauchinhaltsstoffe, die offenkundig nicht aufgeführt sind, wie z.B. Dioxine, wurden hinzugefügt und die existierenden HSR Werte von Zigaretten ohne Filter aus den1950er und 1960er Jahren wurden durch Werte ersetzt, die für den gegenwärtigen Zigarettenmarkt repräsentativer sind. Werte der 1R4F Kentucky Referenzzigarette, die unter Standardbedingungen gemäß ISO (International Organization for Standardization) und FTC (Federal Trade Commission) Richtlinien abgeraucht wurde, wurden wenn möglich stellvertretend für die heutige marktübliche Zigarette angeführt.

Eine Liste toxischer Substanzen im Rauch und deren annähernde Mengen in heutigen Zigaretten ist ohne geeignetes Ranking ihrer relativen Toxizität weitgehend nutzlos. Leider stehen toxikologische Daten für ein Ranking nur für weniger als 5\% der ungefähr 4800 bekannten Rauchkomponenten zur Verfügung. Obwohl keiner der Autoren dieser Arbeit sich anmaßt, Toxikologe zu sein, werden in der Diskussion mehrere publizierte Versuche zitiert, ein Ranking der Toxizität des Rauches vorzunehmen. Hierzu zählen insbesondere das Ranking der Occupational Safety and Health Administration (OSHA) in den USA zur erlaubten Höchstmenge von Substanzen am Arbeitsplatz, die Toxizitätskriterien der Environmental Protection Agency (EPA) in den USA mit dem Zusatz der in Kalifornien geltenden EPA Kriterien sowie die Übertragbarkeit von Tierversuchsdaten auf den Menschen und die von Ames et al. entwickelte Datenbank existierender Daten. Es scheint ein großer Unterschied zwischen zulässigen Höchstmengen am Arbeitsplatz und den von Umweltschutzbehörden empfohlenen Höchstmengen zu geben. Es ist demzufolge zu erwarten, dass derartige Rankings letztendlich dazu führen, dass bestimmte toxische Substanzen besondere Berücksichtigung finden, und zwar bei den Produzenten hinsichtlich einer Reduzierung dieser Substanzen als auch bei der Entwicklung analytischer Standardmethoden.

In dieser Übersicht über toxische Substanzen im HSR werden ebenfalls die Grenzen toxikologischer Bewertungen untersucht. Die Toxizitätsdaten der oben genannten Rankings beziehen sich alle auf Studien mit Einzelsubstanzen. Es ist höchst unwahrscheinlich, dass die Ergebnisse aus Tierversuchen mit Einzelsubstanzen extrapoliert werden können, wenn diese Substanz in einem so komplexen Gemisch wie dem HSR einer Zigarette vorliegt. So wurde zum Beispiel in vielen Studien der vergangenen Jahrzehnte berichtet, dass der Benzo $[a]$ pyren $(\mathrm{B} a \mathrm{P})$ Gehalt im Kondensat von Zigarettenrauch (CSC) nur in wenigen Prozent der tumorigenen Wirkung auf der Haut verantwortlich sei. Daraus wurde gefolgert, dass die Tumor verursachenden polyzyklischen aromatischen Kohlenwasserstoffe (PAHs) im CSC für nicht mehr als 3\% bis $4 \%$ der Tumore auf der Haut von Versuchstieren verantwortlich sein könnten. Das Hinzufügen von Promotoren, wie z.B. 
der Phenole, erhöhe die Quote auf bis zu 5\%. Einige derselben Forscher haben jedoch kürzlich behauptet, dass $\mathrm{B} a \mathrm{P}$ eine von zwei Substanzen sei, die bei Rauchern Lungenkrebs verursache.

Während über die ungefähr hundert toxischen Substanzen im Zigarettenrauch viel geschrieben wurde, wurde über die zahlreichen nichttoxischen Rauchsubstanzen, die in vielen Tierversuchen nachweislich den Wirkungen der toxischen Substanzen entgegenwirken, wenig berichtet. In einigen Fällen werden die inhibitorischen Komponenten ebenfalls unter den toxischen Substanzen gelistet, so hemmt z.B. Nikotin die Mutagenität von $N$-Nitrosodimethylamine, der Promotor Phenol hemmt die Tumor verursachende Wirkung von $\mathrm{B} a \mathrm{P}$, das schwach Tumor verursachende Benz $[a]$ anthracen macht die starke tumorigene Wirkung von $\mathrm{B} a \mathrm{P}$ zunichte. Auf einer eins zu eins molaren Basis wirken viele bizyklische, trizyklische und tetrazyklische nicht tumorigene PAHs der tumorigenen Wirkung von $\mathrm{B} a \mathrm{P}$ und Diben$\mathrm{z}[a, h]$ anthracene entgegen.

Um die unklare toxikologische Situation näher aufzuzeigen, wird ein Überblick über die Geschichte und das gegenwärtige Wissen zur Bedeutung tabakspezifischer Nitrosamine (TSNAs) für die Risiken des Rauchens gegeben. Kurz gesagt, wurden diese Substanzen in Tabakprodukten entdeckt und es wurde festgestellt, dass sie in den HSR (und Nebenstromrauch) übergehen. Toxikologische Beurteilungen der reinen Substanzen haben gezeigt, dass es sich um starke Karzinogene handelt. Einige Wissenschaftler des Öffentlichen Gesundheitswesens vertraten die Ansicht, dass die Verringerung der TSNA Mengen im HSR zu einer „weniger schädlichen“Zigarette führen würde. Nach dieser Festlegung haben Agrarwissenschaftler entdeckt, dass die TSNA Mengen zumindest bei flue-cured Tabaken durch die Verwendung indirekter Heizsysteme in den Trockenschuppen stark verringert werden können. Dieses waren wunderbare Neuigkeiten. Toxikologen führten bald Untersuchungen durch, in denen die Toxizität des HSR von fluecured Tabaken mit hohen und sehr niedrigen TSNA Konzentrationen miteinander verglichen wurde. Diese Forscher müssen überrascht gewesen sein festzustellen, dass in der Toxizität des Rauches beider Tabake kein signifikanter Unterschied bestand.

Einige Wissenschaftler des Öffentlichen Gesundheitswesens haben behauptet, dass die Verringerung des Kondensatgehalts pro Zigarette unter $15 \mathrm{mg}$ das mit dem Rauchen verbundene Risiko nicht vermindert, da die erhöhte Zugabe von Additiven zur Aufrechterhaltung der Akzeptanz des Rauchers eine Gesundheitsgefahr darstelle. Während in der Vergangenheit bisher keine Daten präsentiert wurden, die diese Behauptung stützen würden, wurden im vergangenen Jahrzehnt viele Ergebnisse erhalten, die dieser Behauptung widersprechen. Die Zugabe von Zusatzstoffen in gewöhnlicher oder mehrfach erhöhter Menge führt zu leichten Veränderungen in der Rauchchemie, diese Veränderungen haben jedoch, wie in verschiedenen Tierversuchen nachgewiesen, in denen die Mutagenität oder Tumorigenität usw. untersucht wurde, keine nachteiligen biologischen Reaktionen zur Folge.

Aus unserer Übersicht der Literatur kommen wir $\mathrm{zu}$ folgenden Schlussfolgerungen:

1 Es ist möglich, eine Liste der bekannten toxischen Substanzen im HSR zusammenzustellen und einige dieser Substanzen aufgrund existierender biologischer Daten als besonders toxisch zu klassifizieren. Für mehr als 95\% der bekannten Komponenten des HSR sind jedoch keine biologischen Daten bekannt.

2 Auch wenn es biologische Daten für die meisten Inhaltsstoffe des HSR gäbe, wäre eine Extrapolation der toxischen Eigenschaften der Einzelsubstanzen auf die biologischen Eigenschaften eines Gemisches, das diese Substanzen enthält, außerhalb unserer wissenschaftlichen Fähigkeiten.

3 Auf der Basis unserer heutigen wissenschaftlichen Kenntnisse wird niemand berechtigterweise die Entwicklung einer ,weniger schädlichen Zigarette“ fordern können, die auf einer alleinigen Reduzierung bekannter toxischer Substanzen im HSR beruht.

4 Der Ansatz, den Kondensatgehalt von Zigaretten zu reduzieren, erscheint rückschauend betrachtet der praktikabelste Weg zur Herstellung einer ,weniger schädlichen“ Zigarette zu sein, weil bei einer Verringerung des Kondensatgehalts durch den Produzenten sowohl die bekannten als auch die unbekannten toxischen Substanzen reduziert werden.

5 Das Ranking toxischer Substanzen im HSR enthält sowohl Gasphasen- als auch semivolatile Substanzen, die für die Toxizität von entscheidender Bedeutung zu sein scheinen. Einige dieser Komponenten, z.B. $N$ Nitrosodimethylamin und die Phenole werden durch mit Triacetin behandelten Zelluloseacetatfiltern reduziert. Diese Filter verringern ebenfalls den Kondensatgehalt. Außerdem ist bekannt, dass Carbonylverbindungen durch Aktivkohlefilter sehr wirksam aus dem HSR entfernt werden. Die Entwicklung von mehr Produkten, die vom Konsumenten akzeptiert werden und bei denen toxische Substanzen in der Gasphase reduziert sind, scheint ein weiterer Weg zu einer, ,weniger schädlichen" Zigarette zu sein.

[Beitr. Tabakforsch. Int. 20 (2003) 481-545]

\section{RESUME}

Les temps sont curieux. Le gouvernement canadien a établi une loi exigeant des tests réguliers et la publication des teneurs de 44 substances toxiques dans la fumée du courant principal de la cigarette $(\mathrm{CP})$ par les producteurs de cigarettes. Suivant l'exemple de leur voisin du Nord, les législateurs et l'administration des Etats Unis envisagent de modifier les tests sur les cigarettes et les méthodes d'information, exigeant également l'évaluation des substances toxiques du CP. De l'autre côté de l'océan atlantique, la Commission Européenne a soumis une directive qui pourrait également suivre l'exemple de l'Amérique du Nord, en exigeant de rendre public les substances toxiques du CP pour toutes les marques de cigarettes commercialisées. Les autorités du Royaume Uni ont également exprimé leur intention de suivre ce mandat.

Il est difficile de comprendre la motivation et la valeur de ces actions législatives potentielles ou ultérieures. Bien qu'il y ait presque un accord unanime entre les chercheurs du monde entier que fumer des cigarettes présente un risque sanitaire pour le fumeur, peu peuvent affirmer avec crédibilité, quels sont les composés chimiques, ou classes de composés, de la 
fumée responsable d'effets néfastes sur la santé. Par conséquent, si même les spécialistes ne sont pas capables d'interpréter clairement les données toxiques de la fumée, comment le public va-t-il utiliser ces nouvelles informations ? La compilation des données sur les composants toxiques de la fumée pose également des problèmes à l'industrie du tabac pour plusieurs raisons. Premièrement, il n'y a pas de procédures analytiques standard pour la plupart des composants supposés être toxiques. Deuxièmement, la compilation des teneurs en substances toxiques pourrait provoquer une variante du $21^{\text {ième }}$ siècle de la «guerre du goudron » des années 1960 aux Etats Unis ; nous avons déjà observé l'existence d'une telle compétition démarrant aux Etats Unis. Troisièmement, et ce qui importe avant tout, personne ne sait si la réduction de la teneur en un seul ou plusieurs composants toxiques du $\mathrm{CP}$ produira une cigarette « moins dangereuse ».

En supposant que la situation actuelle se présente environ comme décrit ci-dessus, les auteurs de cette revue ont étudié à fond les listes existantes sur les composants toxiques du CP. Ils ont exclu les composants qui ne sont plus pertinents, tels que le DDT et le $N$-nitrosodiethanolamine, ajouté des composants connus qui sont manifestement absents, tels que la dioxine, et ont substitué les teneurs du CP de cigarettes sans filtre des années 1950-60 avec les données de cigarettes plus représentatives du marché actuel. Les données de la cigarette de référence 1R4F, fumée sous les conditions normalisées ISO (International Organization for Standardization) et FTC (Federal Trade Commission) sont utilisées quand cela est possible comme substitut de la cigarette actuelle.

Une liste des composants toxiques et leurs concentrations approximatives dans les cigarettes commercialisées est pratiquement inutile sans évaluation appropriée de la toxicité relative de chaque composant. Malheureusement, les données toxiques permettant une évaluation ne sont disponibles que pour moins de 5\% seulement des 4800 composants environ rapportés. Bien que les auteurs ne présument pas être toxicologistes, ils rapportent dans la discussion sur plusieurs essais publiés de l'évaluation des composants toxiques de la fumée. En particulier, l'évaluation de l'exposition maximale sur le lieu de travail établie par l'Occupational Safety and Health Administration (OSHA) aux Etats Unis, l'utilisation des critères toxicologiques de l'Environmental Protection Agency (EPA) aux Etats Unis avec en supplément des critères de l'EPA en Californie, l'utilisation de la méthodologie exposition humaine - potentiel chez les rongeurs, et, si des données sont disponibles, base de données développée par AMES et al. La divergence entre les expositions admissibles sur le lieu de travail et les concentrations recommandées par des réglementations de l'environnement semble être grande. Ainsi, il est attendu que de telles évaluations attirent une attention particulière sur des composants chimiques particuliers, soit en vue d'une réduction de la part du producteur, soit en vue d'un développement de méthodes analytiques standardisées.

Cette revue des composants toxiques du $\mathrm{CP}$ examine également les limites d'évaluations toxicologiques. Toutes les données toxiques utilisées dans l'évaluation ci-dessus ont été obtenues par des études de substances uniques. Il est particulièrement improbable qu'une extrapolation des résultats obtenus d'une substance unique par des tests biologiques puisse être faite à la même substance, si cette substance est la composante d'un mélange aussi complexe que le CP. Au cours de plusieurs décennies, de nombreux chercheurs ont rapporté que la teneur en benzo[ $a]$ pyrène $(\mathrm{B} a \mathrm{P})$ du condensat de fumée de cigarette (CSC) explique quelques pourcentages seulement des formations tumorales chez les animaux dans les tests biologiques, comprenant l'application d'un composant sur la peau des animaux. Par la suite, ils ont affirmé que les hydrocarbures polycycliques aromatiques $(\mathrm{PAH})$ tumorigènes du $\mathrm{CSC}$ ne pouvaient pas être responsables que de plus de $3 \%$ ou $4 \%$ des formations tumorales chez les animaux. L'inclusion de promoteurs, tels que les phénols, augmente le taux à environ 5\%. Cependant, quelques-uns de ces mêmes chercheurs ont récemment prétendu que le $\mathrm{B} a \mathrm{P}$ est un des deux composants de la fumée responsable du cancer du poumon chez les fumeurs.

Tandis que beaucoup a été publié sur à peu près cent composants toxiques de la fumée de cigarette, il y a rarement de publications sur les nombreux composants non-toxiques, qui ont montré un effet d'inhibition des composants toxiques dans plusieurs tests biologiques. Dans certains cas, les composants inhibiteurs figurent également parmi les composants toxiques, la nicotine inhibe par exemple la mutagénicité de $N$-nitrosodiméthylamine ; le promoteur phénol inhibe la tumorigénicité de $\mathrm{B} a \mathrm{P}$; le benz $[a]$ anthracène, légèrement tumorigène, annule la forte tumorigénicité de $\mathrm{B} a \mathrm{P}$. Sur une base molaire « one-toone », plusieurs PAH bicycliques, tricycliques et tetrecycliques non-tumorigènes inhibent la tumorigénicité de $\mathrm{B} a \mathrm{P}$ et dibenz $[a, h]$ anthracène.

Pour illustrer cette situation toxicologique, l'historique et la connaissance actuelle sur l'importance des nitrosamines spécifiques du tabac (TSNA) pour les risques sanitaires du fumage sont passés en revue. En bref, ces composants ainsi que leur transfert dans le CP (et le courant secondaire) ont été découverts dans les produits du tabac. Les évaluations toxicologiques des substances uniques ont montré qu'il s'agit de carcinogènes puissants. Certains chercheurs de la santé publique ont supposé que si les teneurs en TSNA du CP pouvaient être réduites, cela devrait permettre d'obtenir une cigarette « moins nocive ». Après cette déclaration, les agronomes ont découvert que les teneurs en TSNA peuvent être réduites par l'utilisation d'un chauffage indirect dans les séchoirs. Ces nouvelles étaient merveilleuses. Cependant, des toxicologistes ont vite mené des essais pour comparer la toxicité du CP de cigarettes «flue-cured » ayant des teneurs élevées et ultra-réduites en TSNA. Cela a été une surprise pour ces chercheurs de trouver qu'il n'y avait pas de différence significative de la toxicité des deux fumées.

Certains chercheurs de la santé publique ont affirmé que la réduction de la teneur en goudron par cigarette en dessous de $15 \mathrm{mg} / \mathrm{cig}$ ne réduit pas le risque engendré par la cigarette à cause du danger résultant de l'apport plus élevé d'additifs pour sauvegarder l'acceptabilité par le consommateur. Tandis que cette assertion n'a jamais été confirmée par des résultats, beaucoup de données obtenues sont en contradiction avec cette assertion. L'apport d'ingrédients, à des niveaux habituels ou plus élevés, entraîne des changements insignifiants dans la chimie de la fumée, mais, comme cela a été mesuré dans plusieurs tests biologiques pour déterminer la mutagénicité, tumorigénicité, etc., ces 
changements ne conduisent pas à des réactions biologiques négatives.

La littérature examinée pour préparer cette revue nous mène à tirer les conclusions suivantes :

1 Il est possible de préparer une liste des composants toxiques connus du $\mathrm{CP}$ et de prêter une attention particulière à certains d'entre-eux par rapport aux données biologiques. Cependant, pour plus de $95 \%$ des composants du CP, il n'existe pas de données biologiques.

2 Même si des données biologiques existaient pour la plupart des composants du CP, l'extrapolation des données obtenues des substances uniques aux propriétés biologiques d'un mélange contenant ces substances est au-delà de notre capacité scientifique.

3 D'après nos connaissances scientifiques actuelles, il ne sera jamais possible de prétendre au développement d'une cigarette « moins nocive », basée sur la réduction de composants toxiques connus du CP seulement.

4 L'approche qui consiste en une réduction de la teneur en goudron d'une cigarette semble être la méthode la plus pratique pour arriver à une cigarette « moins nocive », parce qu'en réduisant la teneur en goudron, les composants toxiques à la fois connus et inconnus sont réduits.

5 Les substances évaluées comme toxiques du CP contiennent à la fois des constituants volatils et semivolatiles, qui semblent être déterminants pour la toxicité. Certains de ces composants, comme le $N$-nitrosodiméthylamine et les phénols sont réduits par des filtres d'acétate de cellulose plastifiés par le triacétine. Ces filtres réduisent également la teneur en goudron. En plus, il est bien connu que des filtres contenant du charbon éliminent efficacement les composés carbonyle de la fumée. Le développement de plus de produits acceptables pour le consommateur qui réduisent les substances toxiques de la phase gazeuse semble être une voie alternative vers une cigarette « moins nocive ».

[Beitr. Tabakforsch. Int. 20 (2003) 481-545]

\section{INTRODUCTION}

Things should be made as simple as possible, but not any simpler.

$$
\text { - Albert Einstein }
$$

The quotation from Professor Einstein seems extremely appropriate when embarking on any discussion attempting to link cigarette MSS composition to the hazards of smoking. The simplistic dream of both Tobacco Industry and public health scientists is to identify the smoke constituents responsible for adverse health effects and either greatly reduce or eliminate those chemicals to create "less hazardous" products. Whether or not this hope can be made reality is unknown. However, the authors of this review can state with some certainty that the current status of chemical and toxicological sciences does not allow us to demonstrate that any specific chemicals or classes of chemicals present in tobacco smoke are responsible for the health hazards of smoking. Our current belief is in concert with that expressed earlier by the National Research Council of the National Academy of Sciences (1) regarding the status of knowledge relating smoke composition with health hazards.
Even after decades of serious investigation, we do not understand the role of tobacco smoke components in producing chronic diseases, such as arteriosclerosis, emphysema and malignant neoplasms. The task of identifying the toxic components is overwhelming and must be considered currently impossible. However, we can identify groups of agents from a knowledge of their chemical similarity to agents generated in a standard control substance.

Soon following the pioneering epidemiological research relating smoking to carcinoma of the lung by WYNDER and GRAHAM in the USA and DOLL and HILL in the UK (2) and the generation of tumors in mice following skin painting with smoke condensate by WYNDER et al. (3), chemists have been attempting to answer the question as to what components in tobacco smoke are responsible for the reported findings. Prior to 1954, tobacco smoke was recognized as an extremely complex mixture but very little was known about its composition. Fewer than 100 components had been reported, but many identifications were subsequently shown to be incorrect. As reviewed by GREEN and RODGMAN (4), among the first published lists of tobacco smoke constituents was that of KOSAK (5). His list contained approximately 80 entries of which almost one-half were questionable in regards to correct identity. GREEN and RODGMAN (4) estimated that there are approximately 4800 known components in tobacco smoke.

Lists of toxic components in tobacco smoke are also not new phenomena. Among the first of these lists was one contained in the 1964 report of the ADVISORY COMMITTEE to the US Surgeon General (6) on smoking and health. It seems as though there has been either a new list of MSS toxicants published or a re-publication of a previously published list every year since the first list appeared. Perhaps most notable among the smoke toxicant list publications is the "List of 43" prepared by HOFFMANN and HECHT (7). This list was used by the US Environmental Protection Agency (EPA) (8) to bolster their argument that exposure to second-hand cigarette smoke is a cause of lung cancer in nonsmokers. RODGMAN (9) has pointed out the deficiencies in the "List of 43" and interpretations made by the EPA. Within the last few years, additional and/or revised lists of smoke toxicants have been published by BAKER and PROCTOR (10), HofFMANN et al. (11), HofFMANN and HofFMANN (12), and SMiTH et al. (13-15). Although the cited lists and others not presented herein contain much valuable information, they tend to perpetuate data contained in the earliest publications that are unsupportable, incorrect and irrelevant to the current situation.

Even though the emphasis over the years on all but one class of toxicants has waxed and waned, it has become common practice since the mid-1980s to publish lists of cigarette smoke toxicants and their per cigarette deliveries. With time, the toxicant lists have become longer and longer. Because they are, by definition, tobacco-specific, the TSNAs are the one component class that still remains in the limelight. Interestingly, the identification of PAHs in MSS progressed from a few in the 1950s to more than 500 in the 1970s. That situation differed greatly from the $N$ nitrosamine (NNA) case where little research on additional NNAs in MSS was conducted once the tobacco-specific NNAs were identified.

In several instances, listed toxicants have 1) no identified precursor in tobacco, 2) no quantified MSS levels, 3) a 
possible artifactual origin, or 4) an unconfirmed presence. Some cigarette MSS components considered toxic when encountered environmentally, e.g., the dioxins, are not included in any of the cigarette smoke toxicant lists. Also interesting is the fact that some toxicants for which no or only a few quantitative data are available are given equal weight to other toxicants for which literally hundreds of quantitative data have been generated since the mid-1950s, cf. the hundreds of publications on the MSS level of $\mathrm{B} a \mathrm{P}$ vs. the few that only list dibenzo $[a, l]$ pyrene or benzo $[b] \mathrm{fu}-$ ran as present.

The oft-repeated assertions [see review by RoDGMAN (16)] that ingredients (flavorants, casing materials, humectants) added to tobacco enhance the levels of cigarette MSS toxicants as well as the adverse biological effect of MSS are without merit. No individual or agency making such claims has ever presented detailed data to support such assertions. On the other hand, considerable laboratory evidence has been generated to discount both the adverse compositional and biological assertions (17-23).

Whether or not a chemical constituent is included in the list of smoke toxicants appears to be the result of a haphazard process. There are at least two types of smoke toxicant lists that may be useful. One is a historical record of every toxic component that has been identified, and a second, more useful list related to the development of a "less hazardous" cigarette, would contain all known toxic compounds found in currently-sold commercial cigarette MSS. For both lists, criteria should be stated up front documenting justification for including a smoke constituent. Among these criteria should be the following:

- Confirmation of identity by more than one researcher or research group

- Documentation of carcinogenic properties by the International Agency for Research on Cancer (IARC), US EPA, National Toxicology Program, etc.

- Documentation of other toxic properties by appropriate agencies or scientific studies

- An assessment of the quantification method and the quality of the analytical result. From a risk assessment perspective, qualitative identification of smoke toxicants is practically useless.

As you will note from the title of this paper, the authors have used the term hoopla in reference to reports of toxic chemicals in cigarette MSS. Hoopla is defined by WEBSTER (24) as "excited commotion". As RICKERT and KAISERMAN have already pointed out (25), "Surveys of Canadian smokers have demonstrated that their [sic] continues to be confusion regarding the meaning of the numbers for "tar," nicotine and CO which appear on every package of Canadian cigarettes" (26,27). Because smokers cannot understand "tar" and nicotine labeling, it is difficult to understand how they will interpret toxic compound data. Thus far in antismoking efforts, it appears that at least one of the prime uses of a toxic substance list is the production of public service advertisements. These pronouncements, e.g., the one in 1998 by the National Center for Tobacco Free Kids (28), of the dangers of smoking tend to focus on commonly known toxicants such as arsenic. Rarely mentioned are such substances as $\mathrm{B} a \mathrm{P}$ or 4- $(N$-methylnitrosamino)-1-(3-pyridinyl)-1-butanone (NNK) because the general public has no recognition of these terms.
In recent years a trend to integrate quantitative risk assessment into the listing of smoke toxicants has emerged. These techniques rely on relative toxicity values published by the US Occupational Safety and Health Administration (OSHA), US EPA, IARC, etc. and ultimately allow a relative order of potential harm ranking of the known smoke toxicants. This process has severe limitations among which are the following:

- The smoke yield data for many known/suspected smoke toxicants are of unknown quality. This problem has been exacerbated by the introduction of human smoking conditions into the analytical process. Additionally, few of the reported smoke yields have been determined by validated analytical methods.

- For the reported 4800 smoke constituents, there are existing toxicity data for fewer than $5 \%$ of the compounds.

- As Rodgman (9) has pointed out, mainstream tobacco smoke contains many inhibitors, anticarcinogens, and antimutagens that must be accounted for in assessing the potency of an individual chemical or class of chemicals in cigarette smoke.

and the most significant deficiency of all

- All of the data for smoke toxicants come from animal studies on individual compounds. Prediction of complex mixture toxicology from data on individual components as well as prediction of the toxicology of an individual component in a complex mixture is beyond the current capability of science.

However, if progress is to be made in relating MSS composition to adverse health effects of smoking, quantitative risk assessment of smoke toxicants may be a necessary first step in identifying the relative importance of compounds. Additionally, as SAINT-JALM (29) recently stated concerning the development of validated analytical methods, “. . . there is need to set criteria in order to select which methods should be developed as a priority and it is the intention of CORESTA to work in this direction". Quantitative risk assessment may be a beginning approach to selecting target compounds.

Developing a quantitative risk assessment for MSS toxicants may be beyond the realm of scientific competency for this paper's authors because neither is a degreed or certified toxicologist. However, published relative toxicity assessments by R.J. Reynolds Tobacco Company toxicologists (30), VORHEES et al. (31), RICKERT and KAISERMAN (25), TRICKER (32), and FOWLES and BATES (33) serve as our guide in this endeavor.

The study of over forty design technologies to control the delivery and composition of cigarette MSS eventually led to the discovery, development, and use of a few significant ones (34). None of the significant technologies was an outgrowth of various "less hazardous" cigarette activities sponsored by non-Tobacco Industry institutions. All were a product of US Tobacco Industry efforts and were part of commercial cigarette design before the first experiments were conducted in the National Cancer Institute (NCI) Smoking and Health Program on the "less hazardous" cigarette (35). Since the mid-1950s, the use of these technologies in cigarette design either individually or in concert has resulted in the gradual reduction in the levels of many of both the particulate- and vapor-phase toxicants in 
cigarette MSS. Unfortunately, the listed per cigarette delivery range of a particular toxicant often includes data collected on the MSS from commercial cigarettes manufactured in the 1950s and 1960s. Thus, the listed range in terms of the deliveries of MSS components from more recently manufactured cigarettes is unrealistic.

The US Tobacco Industry has recently been criticized because it has introduced no significant new cigarette design technology since 1975 (12). Examination of the annual sales-weighted average "tar" yield for US commercial cigarettes [cf. Figure 3 in RODGMAN (34)] reveals that by the late 1960 s the $40 \%$ to $50 \%$ reduction in MSS "tar" yield, i.e., a reduction from 38-39 mg/cig to $19-20 \mathrm{mg} / \mathrm{cig}$, attained and surpassed the goal originally proposed by WYNDER in 1957 to resolve the lung cancer situation (36). Overlooked by the critics is the fact that the eight significant technologies used in concert and to different degrees have resulted in an additional $40 \%$ reduction to about 12 $\mathrm{mg} / \mathrm{cig}$ in the sales-weighted Federal Trade Commission (FTC) "tar" delivery from 1975 to date.

\section{SOME TOXICANTS IN CIGARETTE MAINSTREAM SMOKE - A $21^{\text {st }}$ CENTURY LIST}

The genesis of our cigarette MSS toxicants list contained in Table 1 originates from the private files of the paper's authors. We have also borrowed liberally from smoke toxicant lists cited earlier in this paper, i.e., references 6 through 15. The table contains 149 entries of which we have highlighted eight (in bold font). The highlighted entries are toxicants that continue to be found in many compilations that have either insufficient evidence of their existence in smoke or are components that have been discontinued in tobacco agronomy for decades and appear to be irrelevant to modern cigarettes. In their recent review, BAKER and PROCTOR (10) initiated the practice of specially designating these smoke components and it is our hope that the compounds will disappear from future lists unless their presence is more firmly documented. We have left these compounds in our list so that we can discuss them and, where toxicity data are available, evaluate the relative harm potential of the substance.

Unfortunately, in including compounds in Table 1, we have not followed our own advice of developing specific criteria to either accept or reject a smoke toxicant from the list. This is a large task in itself and would have broadened the scope of our endeavor to expand beyond both the presentation and publication limits of our current assignment. However, we do recommend that CORESTA undertake this criteria-setting approach as a future work item.

As a substitute for criteria setting, we have included in Table 1 four items which influenced selection of smoke toxicants. These (designated by " $X$ " in Table 1) include the following:

- Listing in the 1993 US Consumer Product Toxicity Testing Plan, 19 toxicants

- Listing in the Canadian Government Testing Protocol, 46 toxicants

- Carcinogenicity classification by IARC, 83 toxicants

- Listing in the US EPA tables as hazardous chemicals for Toxic Chemical Release Inventory, 92 toxicants.
Instead of listing the historically determined minimum and maximum yield of a MSS toxicant, we have chosen to use the yield for the Kentucky reference 1R4F cigarette. Many of the yields listed in previous toxicant lists are from nonfiltered cigarettes of 1950s and 1960s vintage. Because more than $90 \%$ of today's smokers consume filtered cigarettes, values from nonfiltered cigarettes of a past era are not appropriate for analysis. Table 1 lists five primary sources of cigarette yields. These include 1R4F data from Rickert at Labstat International, Inc., R.J. Reynolds Tobacco Company (RJRT) yields either published or on the ECLIPSE cigarette website, and yields published by Vector Tobacco Company on the OMNI cigarette website. When there were multiple instances of 1R4F yields, the highest value was chosen to be the "Comparison Cigarette Value". If $1 \mathrm{R} 4 \mathrm{~F}$ data were not available from these sources, yields of INBIFO control cigarettes were chosen as representative of current commercial cigarettes. If none of the previously cited sources had data on listed smoke toxicants, then the maximum value reported by HOFFMANN et al. was used for further analysis. And finally, a variety of miscellaneous sources were used to obtain cigarette mainstream yield data, when the five primary sources did not produce results. The references to all the data sources are contained within the Table References. All further analyses that required a cigarette MSS yield used the value in the "Comparison Cigarette Value" column.

\section{SMOKING-MACHINE YIELDS IN TABLE 1 FOR 1R4F CIGARETTE}

Although the authors of this paper are aware of the existing controversy concerning proper analytical smoking-machine methodology for determining "Comparison Cigarette Smoke Yields", e.g., BAKER (37), we chose to use yields generated by the existing US FTC or International Standards Organization (ISO) methods. Among the reasons for this choice are the following:

- There exist few data for yields under alternate smoking regimes.

- There is no agreement as to which alternate smoking regime best represents human smoking.

- Although some public health advocates state that existing standard methods underestimate human smoking yields, no smoking-machine methodology takes into account the actual retention of any smoke analyte.

- In terms of quantitative risk assessment, the science is so crude that orders of magnitude changes in smoke yields are necessary to make a significant difference in the outcome of the analysis.

\section{OBSOLETE SMOKE TOXICANTS}

The only non-tobacco specific nonvolatile $N$-nitrosamine identified in tobacco and tobacco smoke is $\mathrm{N}$-nitrosodiethanolamine (NDELA) (IARC, 38). Its presence in tobacco more than two decades ago was related to the use of the sucker growth inhibitor, the diethanolamine salt of maleic hydrazide. Because of a 1981 ban on its use in tobacco agronomy by EPA (39), the diethanolamine salt 


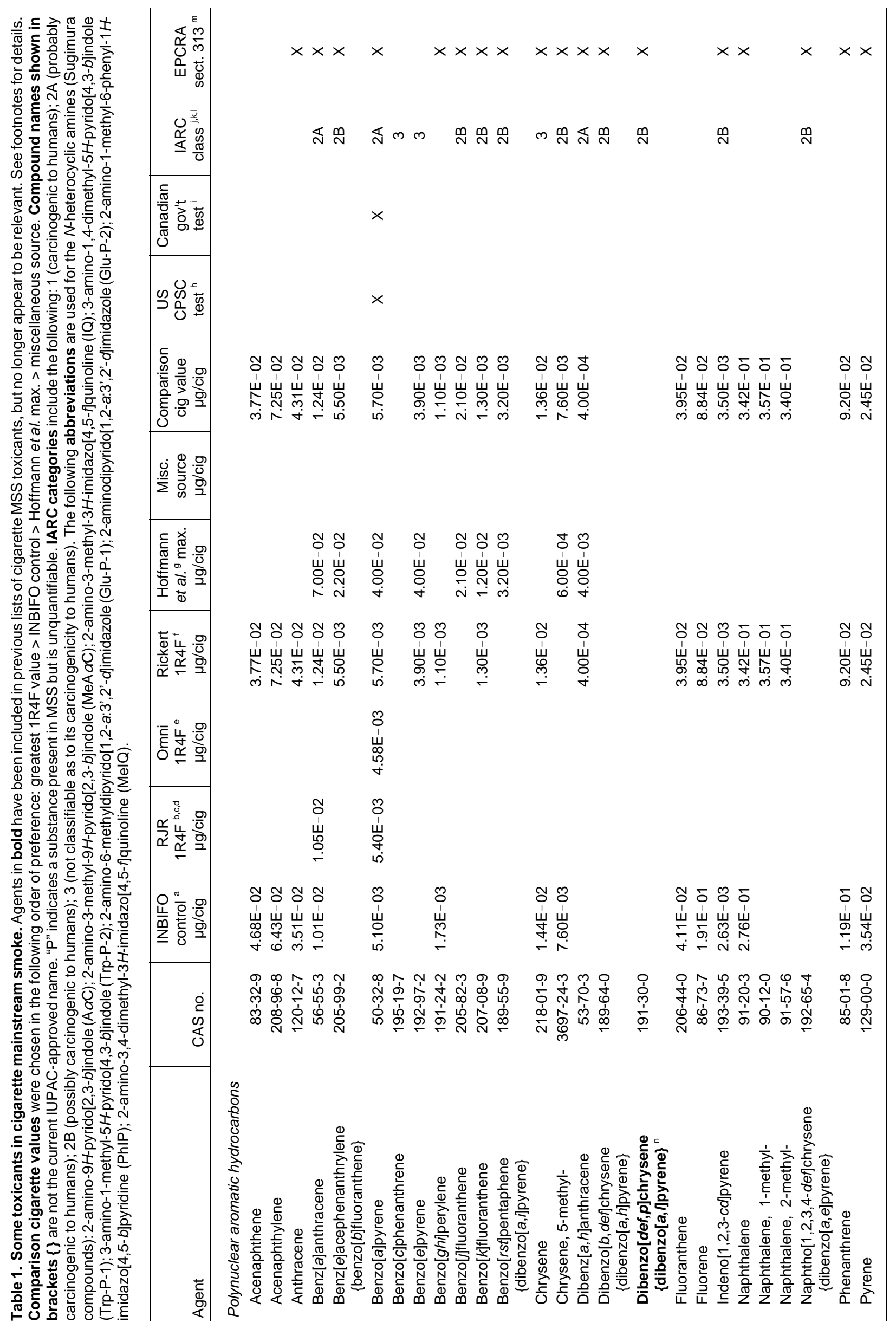




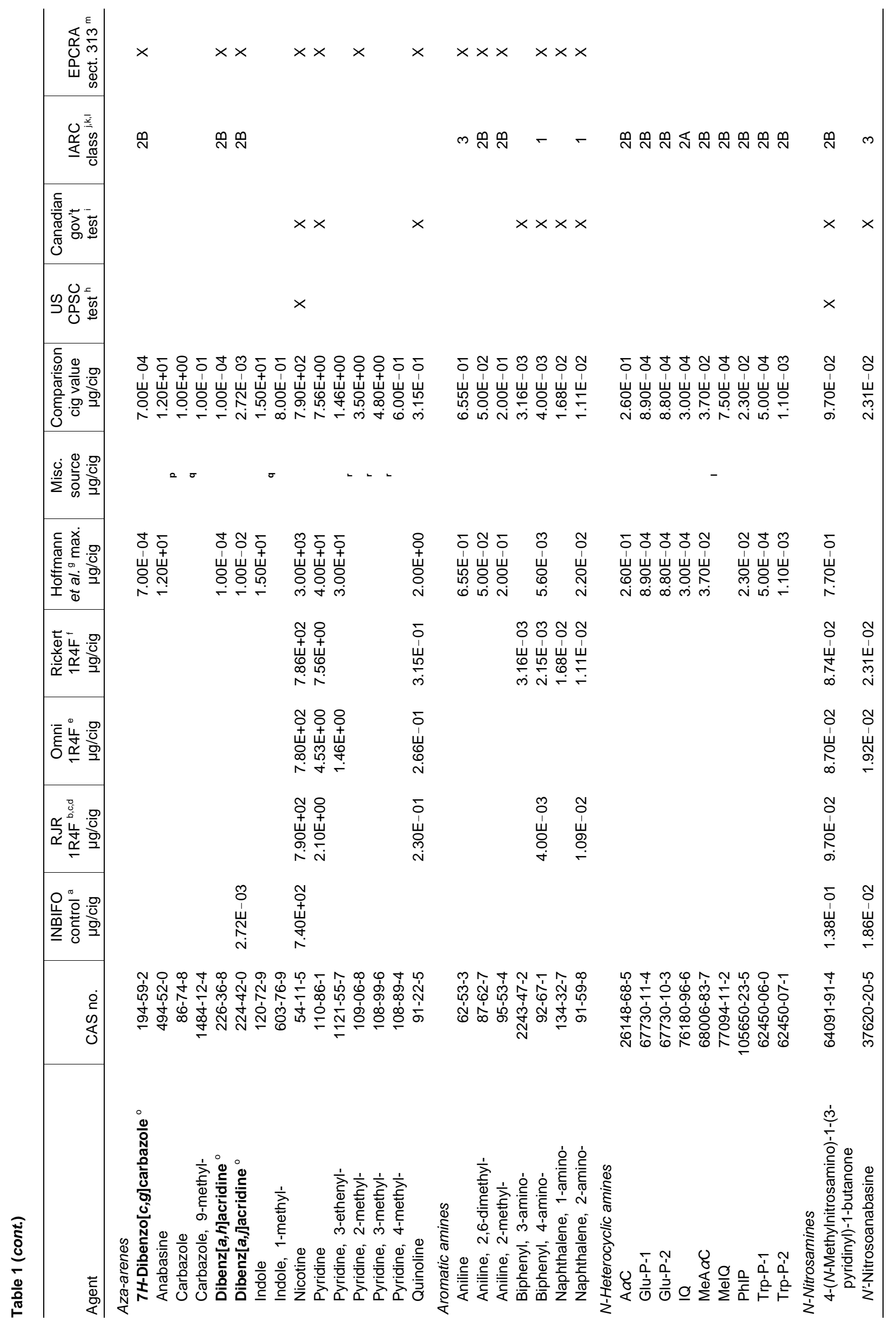




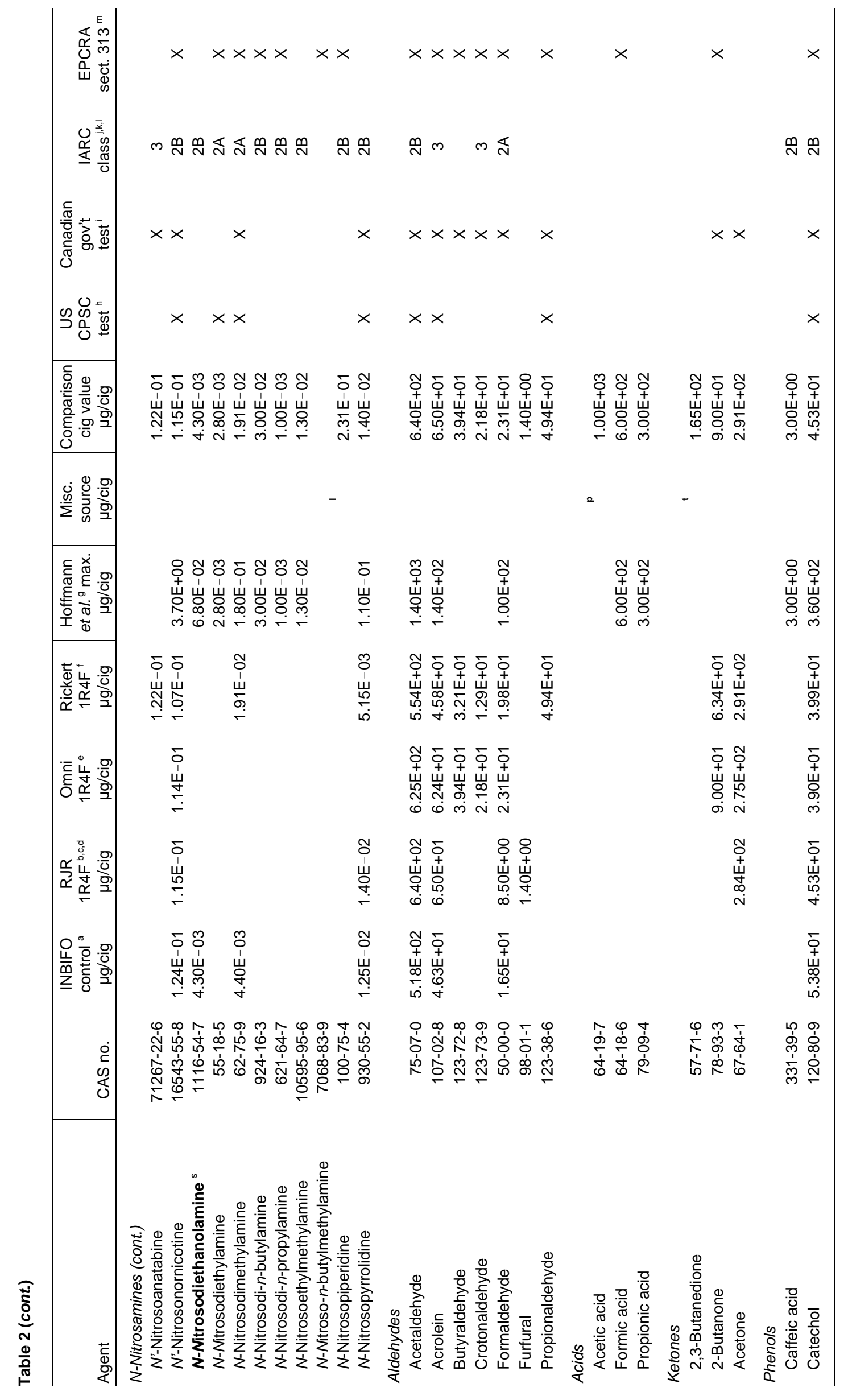




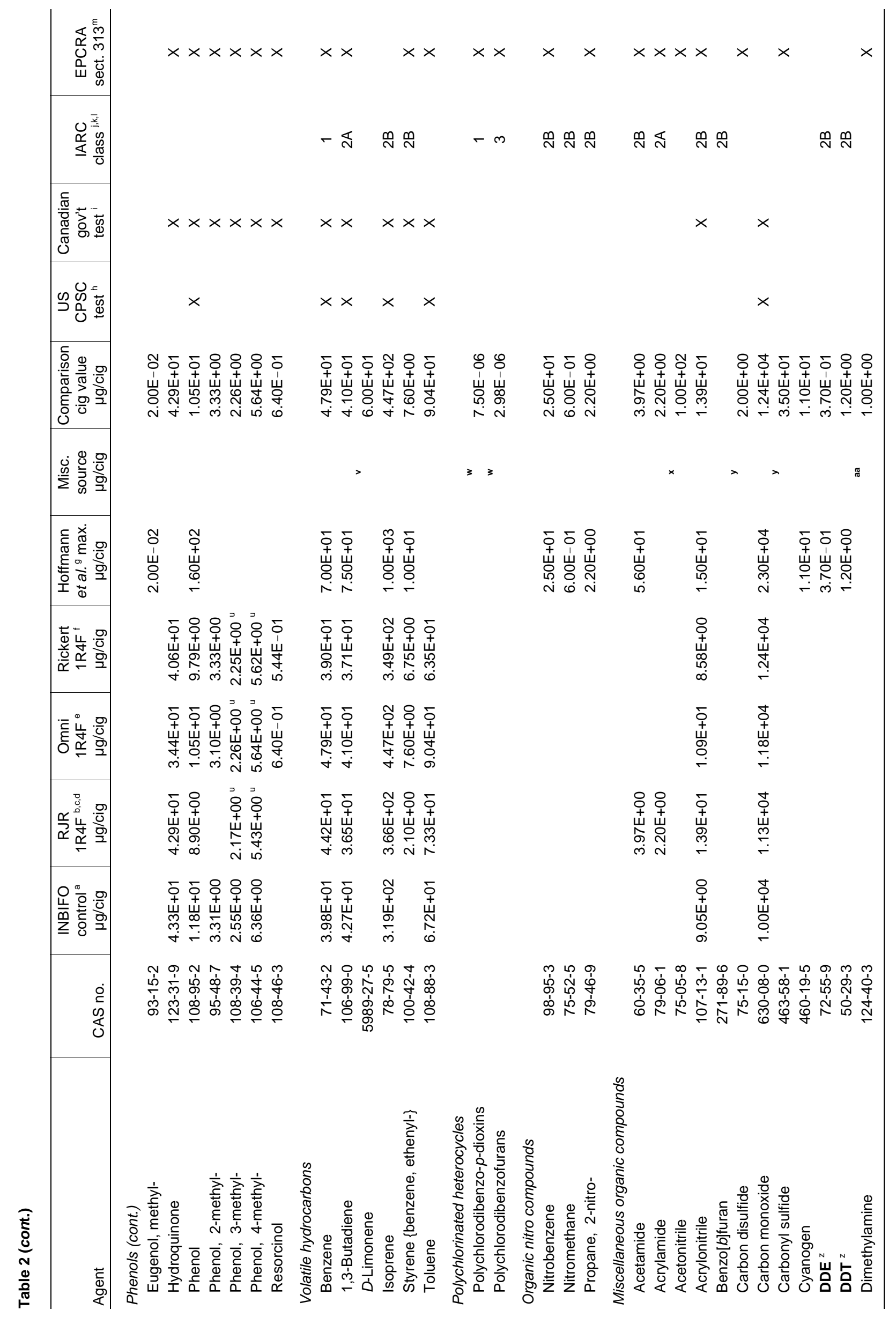




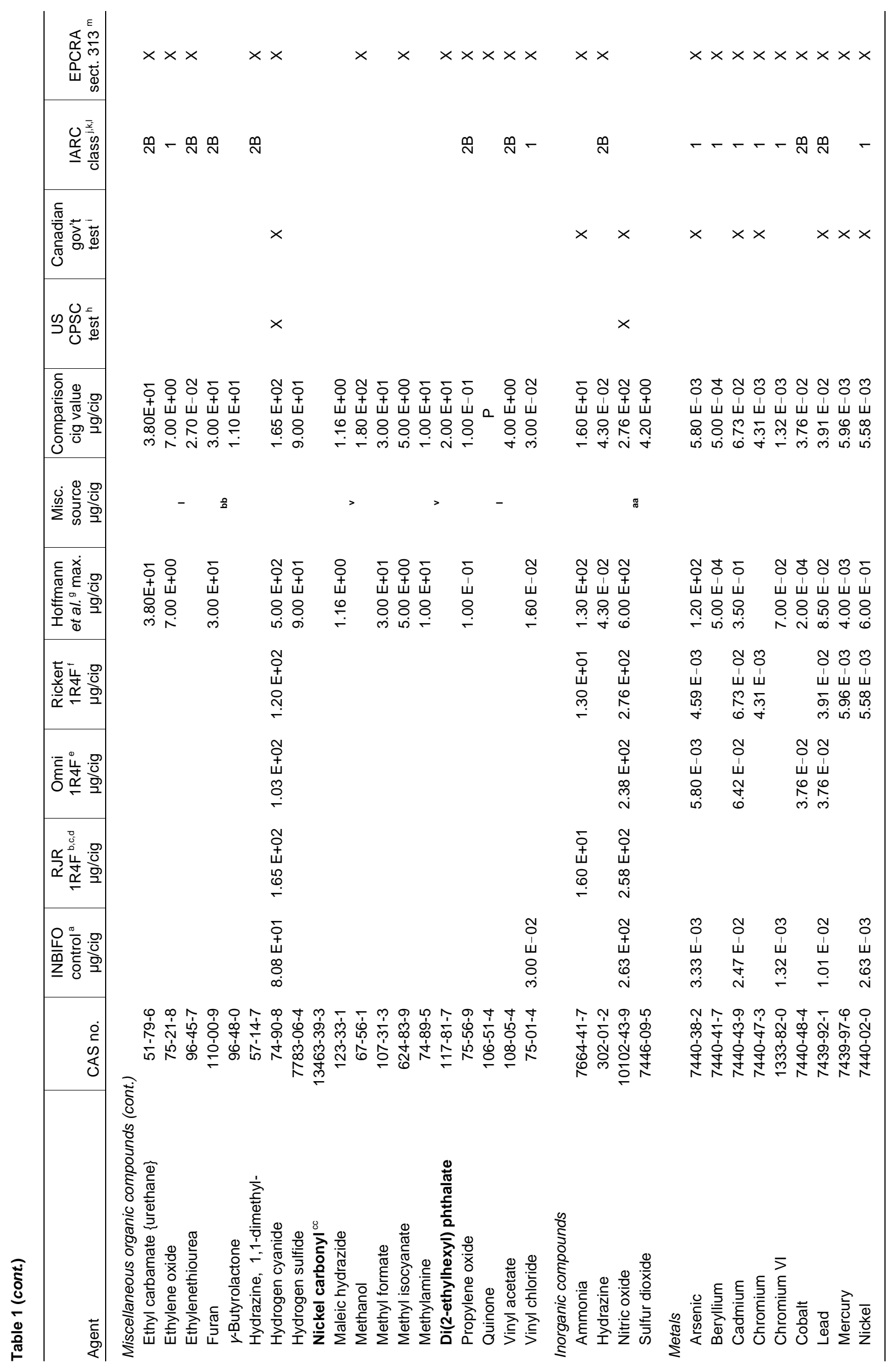




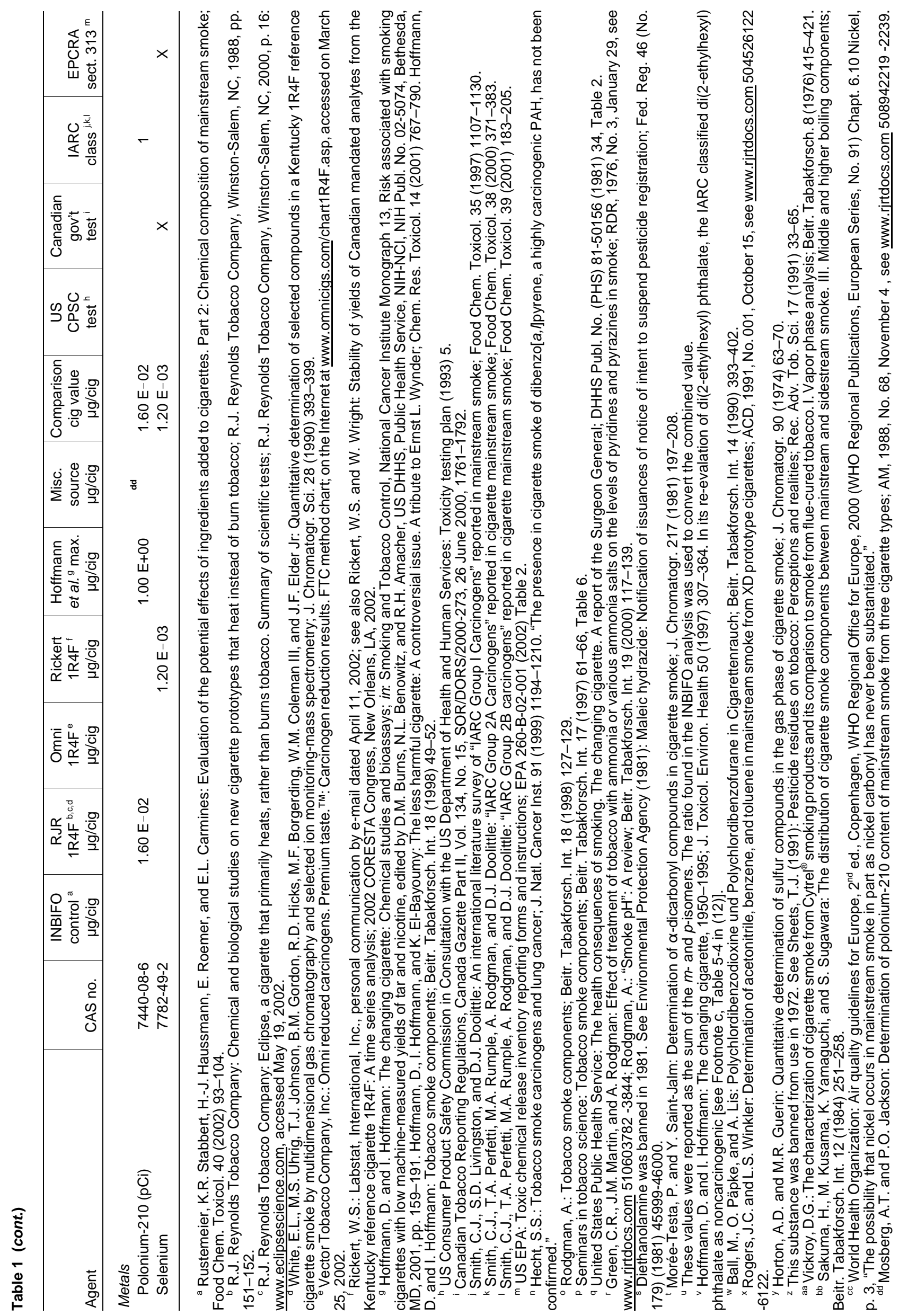




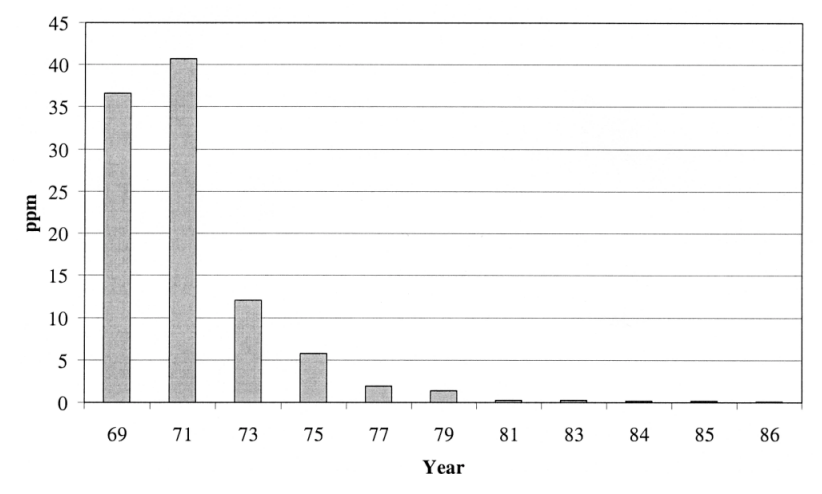

Figure 1. Historical trend of TDE + DDT residues in US cigarettes $(\mathrm{ppm})$

has been replaced by the potassium salt of maleic hydrazide. Analyses of tobacco grown under inhibitor-free conditions and the smoke generated from such tobacco failed to show the presence of NDELA (BRUNNEMANN and HOFFMANN, 40).

Despite the fact that in 1984 HoFFMANN et al. (41) predicted that NDELA in tobacco and its smoke would decrease due to the 1981 ban on the use of the diethanolamine salt of maleic hydrazide and their prediction has come true since 1981, HOFFMANN and his colleagues persist in listing NDELA as a significant tumorigen or biologically active component of cigarette MSS. As reviewed by RODGMAN (42), the diminution of levels of NDELA in tobacco should parallel the chronicled decrease in arsenic and DDT levels in tobacco after these materials were no longer used in tobacco agronomy. Between 1968 and 1974, the residual level of DDT in USA grown fluecured tobacco decreased from a range of 28 to $52 \mu \mathrm{g} / \mathrm{g}$ in 1968 to $6 \mu \mathrm{g} / \mathrm{g}$ in 1970 to $0.23 \mu \mathrm{g} / \mathrm{g}$ in 1974 [USPHS (43), see p. 61; IARC (44)]. In the late 1960s, the transfer rate of DDT from cigarette tobacco to its MSS was reported as 5\% by NESEMANN et al. (45) and as $12 \%$ by HOFFMANN et al. (46). With these percent transfers and a cigarette tobacco level of $0.23 \mu \mathrm{g} / \mathrm{g}$, the MSS would contain either 11 or 28 $\mathrm{ng} / \mathrm{cig}$ of DDT. In the 1979 Surgeon General's report it was noted that a significant reduction of the use of chlorinated hydrocarbon insecticides resulted in reduced residues on the tobacco (43). In a 1979 review, SHEETS and LEIDY (47) reported that the average DDT level in US flue-cured tobacco was $0.13 \mu \mathrm{g} / \mathrm{g}$. Later, Tso (48) summarized most of these data. In 1991, SHEETS (49) summarized some unpublished data on DDT in US commercial cigarettes: The amounts of DDT (sum of the three isomers) in 19 commercial cigarette brands ranged from 0.11 to $0.28 \mu \mathrm{g} / \mathrm{g}$, averaging $0.19 \mu \mathrm{g} / \mathrm{g}$. The historical trend of DDT levels is shown graphically in Figure 1 (50).

Over time, similar decreases were reported for arsenic residues, usually considered as $\mathrm{As}_{2} \mathrm{O}_{3}$, in tobacco after arsenic use was removed from tobacco agronomy in 1952. Between 1917 and 1951 the arsenic level in tobacco rose from about 12 to $57 \mu \mathrm{g} / \mathrm{g}$ (51). By 1968 the arsenic level in tobacco had decreased from the 1951 value of more than 50 $\mu \mathrm{g} / \mathrm{g}$ to a 1968 value of $0.5-1.0 \mu \mathrm{g} / \mathrm{g}$, a value similar to that reported by GRIFFIN et al. (52). Some of these chronological data were summarized by the US Surgeon General in 1979 [see p. 59 in (43)] and IARC (44). In 1957, CogBILL and HoBBS (53) reported the transfer of arsenic from a cigarette containing $7.1 \mu \mathrm{g}$ of arsenic to its MSS to be $3.5 \%$. With the tobaccos analyzed for arsenic by GRIFFIN et al., the arsenic content of the MSS would range from 0.018 to $0.035 \mu \mathrm{g} / \mathrm{cig}$. In 1968, GUTHRIE (54) reported the arsenic transfer from cigarette tobacco to its MSS varied between $4 \%$ and $12 \%$. In 1990, Tso (48) noted that for most tobaccos at that time the arsenic level was around 0.1 to $0.5 \mu \mathrm{g} / \mathrm{g}$.

\section{UNCORROBORATED SMOKE TOXICANTS}

Among the toxicants listed in bold font in Table 1 are three aza-arenes, i.e., $7 H$-dibenzo $[c, g]$ carbazole, dibenz $[a, h]$ acridine, and dibenz $[a, j]$ acridine. All three of these compounds were first reported in cigarette MSS by VAN DUUREN et al. (55) and subsequently CANDELI et al. [(56), see pp. 373-374, Table VIII-14 in (57)] reported unpublished data indicating that dibenz $[a, j]$ acridine is a smoke toxicant. It has long been known that these three compounds are biologically active. However, as RODGMAN has documented (58), "Despite numerous attempts in Japan, Germany, and the USA between 1960 and 1992 to confirm the presence of these three aza-arenes in cigarette MSS and nicotine pyrolysates, the 1960 findings reported . . . have not been confirmed ..." Additionally, since the Rodgman report, SASAKI and MOLDOVEANU (59) have attempted to resolve the controversy related to the presence of the two dibenzacridines in cigarette MSS. Even through the use of selected ion monitoring gas chromatography-mass spectrometry and dibenz $[a, j]$ acridine $-d_{13}$ as an internal standard, SASAKI and MOLDOVEANU were unable to detect the presence of either dibenzacridine in smoke condensate. Although the absence of any compound in cigarette smoke is impossible to prove, enough modern analytical studies have been performed to remove $7 H$-dibenzo $[c, g]$ carbazole, dibenz $[a, h]$ acridine, and dibenz $[a, j]$ acridine from the list of smoke toxicants without further proof of their existence.

\section{POLYCHLORODIBENZO- $p$-DIOXINS AND POLYCHLORODIBENZOFURANS}

Among the smoke toxicants conspicuous in their absence from all toxicant lists except that of FOWLES and BATES (33) are the polychlorodibenzo- $p$-dioxins (PCDDs) and polychlorodibenzofurans (PCDFs). The presence of dioxins in cigarette smoke was first reported in 1980 by CRUMMETT (60). More recently, there are at least five publications reporting the presence of dioxins (PCDDs and PCDFs) in cigarette tobacco or its MSS. These include in chronological order of publication: MUZO and TAKIZAWA (61), BALL et al. (62), MATSUEDA et al. (63), LÖFROTH and ZEBÜHR (64), and Matsueda et al. (65). Example compounds are shown in Figure 2.

The smoke yield data of MUZO and TAKIZAWA (61) come from a single smoking puff that entirely consumes the cigarette and is clearly not appropriate for our quantitative risk assessment. The mainstream and sidestream data of LöFROTH and ZEBÜHR (64) are derived from only one Swedish cigarette brand. The PCDD and PCDF data in the paper by 
<smiles>Clc1cc2c(cc1Cl)Oc1cc(Cl)c(Cl)cc1O2</smiles>

2,3,7,8-Tetrachlorodibenzo- $p$-dioxin<smiles>Clc1cc2oc3cc(Cl)c(Cl)cc3c2cc1Cl</smiles>

2,3,7,8-Tetrachlorodibenzofuran

Figure 2. Example polychlorodibenzo-p-dioxin and -furan

MATSUEDA et al. (65) are for the amount of these compounds contained in the cigarette tobacco, rather than the smoke. The smoke yield data for the BALL et al. (62) and MATSUEDA et al. (63) experiments are similar. Because the BALL et al. data were collected and analyzed by a wellvalidated method, and the laboratory where the analyses were performed, i.e., ERGO Forschungsgesellschaft $\mathrm{mbH}$, Hamburg, has been accredited by the World Health Organization (WHO) for dioxin analysis, we have chosen those results for further analysis.

The analytical data of BALL et al. represent results from the ten top selling brands in Germany during the fourth quarter of 1989. The ERGO scientists chose to present individual data on each of the tested cigarettes. It should be noted that the most toxic isomer, 2,3,7,8-tetrachlorodibenzo- $p$-dioxin (TCDD) was not detected in any of the samples and additionally, not every isomer present was quantifiable in each product tested. For the purposes of this paper we have summarized the average cigarette data in Table 2.

As may be seen listed in Table 2, the total amount of PCDDs and PCDFs is 7.50 and $2.98 \mathrm{pg} / \mathrm{cig}$, respectively. Incorporating all of the various isomers with their individual toxicities into a risk assessment is difficult. Therefore, toxicologists have determined the absolute toxicity of the most potent congener, i.e., TCDD and related the toxicity of all other congeners to the most potent one. The total toxicity potential of a mixture of PCDDs and PCDFs is expressed as 2,3,7,8tetrachlorodibenzo- $p$-dioxin toxicity equivalents (TEQs). However, among US EPA and WHO scientists, there is disagreement over how to calculate the TEQs. Thus, Table 2 shows mean values of TCDD TEQs of 1.258 and 0.0975 $\mathrm{pg} / \mathrm{cig}$, respectively. For all further calculations, we will use the US EPA value because it is greater and any risk based upon its value may be biased high.

\section{QUANTITATIVE RISK ASSESSMENTS}

When you come to a fork in the road, take it. - Yogi Berra (66)

Heretofore, when tobacco scientists prepared a list of known cigarette MSS toxicants, they have for the most part listed the substances by name, amounts reported in smoke, whether or not it is believed to be a carcinogen, promoter, etc., and various other data. However, this type of listing
Table 2. Polychlorodibenzo- $p$-dioxins and -furans in cigarette mainstream smoke ${ }^{a}$ (results in $\mathrm{pg} / \mathrm{cig}^{\mathrm{b}}$ )

\begin{tabular}{|c|c|c|c|}
\hline Compound & Average & $\begin{array}{l}\text { US EPA- } \\
\text { TEQ }\end{array}$ & $\begin{array}{l}\text { WHO- } \\
\text { TEQ }^{\text {e }}\end{array}$ \\
\hline $\begin{array}{l}\text { 2,3,7,8-Tetra-CDD }{ }^{f} \\
\text { Sum tetra-CDD }\end{array}$ & 0.51 & 0.00507 & \\
\hline $\begin{array}{l}\text { 1,2,3,7,8-Penta-CDD } \\
\text { Sum penta-CDD }\end{array}$ & 0.15 & 0.0145 & \\
\hline $\begin{array}{l}\text { 1,2,3,4,7,8-Hexa-CDD } \\
\text { 1,2,3,6,7,8-Hexa-CDD } \\
\text { 1,2,3,7,8,9-Hexa-CDD } \\
\text { Sum hexa-CDD }\end{array}$ & $\begin{array}{l}0.08 \\
0.06 \\
0.04 \\
0.53\end{array}$ & 0.0528 & $\begin{array}{l}0.00771 \\
0.00600 \\
0.00414\end{array}$ \\
\hline $\begin{array}{l}\text { 1,2,3,4,6,7,9-Hepta-CDD } \\
\text { 1,2,3,4,6,7,8-Hepta-CDD } \\
\text { Sum hepta-CDD }\end{array}$ & $\begin{array}{l}1.61 \\
1.29 \\
2.90\end{array}$ & 0.0290 & 0.0129 \\
\hline Octa-CDD & 3.42 & 0.000342 & 0.000342 \\
\hline $\begin{array}{l}2,3,7,8 \text {-Tetra-CDF }{ }^{9} \\
\text { Sum tetra-CDF }\end{array}$ & $\begin{array}{l}0.19 \\
1.41\end{array}$ & 0.705 & 0.0187 \\
\hline $\begin{array}{l}\text { 1,2,3,7,8/1,2,3,4,8-Penta-CDF } \\
\text { 2,3,4,7,8-Penta-CDF } \\
\text { Sum penta-CDF }\end{array}$ & $\begin{array}{l}0.13 \\
0.04 \\
0.83\end{array}$ & 0.414 & $\begin{array}{l}0.00630 \\
0.0206\end{array}$ \\
\hline $\begin{array}{l}\text { 1,2,3,4,7,8/1,2,3,4,7,9- } \\
\text { Hexa-CDF }\end{array}$ & 0.03 & & 0.00300 \\
\hline $1,2,3,6,7,8-$ Hexa-CDF & 0.05 & & 0.00467 \\
\hline $1,2,3,7,8,9-$ Hexa-CDF & 0.07 & & 0.00650 \\
\hline $\begin{array}{l}2,3,4,6,7,8-H e x a-C D F \\
\text { Sum hexa-CDF }\end{array}$ & $\begin{array}{l}0.05 \\
0.35\end{array}$ & 0.0350 & 0.00471 \\
\hline $\begin{array}{l}1,2,3,4,6,7,8-H e p t a-C D F \\
1,2,3,4,6,7,9-H e p t a-C D F \\
\text { Sum hepta-CDF }\end{array}$ & $\begin{array}{l}0.16 \\
0.04 \\
0.27\end{array}$ & 0.00267 & $\begin{array}{l}0.00157 \\
0.000360\end{array}$ \\
\hline Hecta-CDF & 0.15 & 0.0000154 & 0.0000154 \\
\hline Sum PCDD & 7.50 & & \\
\hline Sum PCDF & 2.98 & & \\
\hline Sum PCDD/PCDF & 10.5 & & \\
\hline TEQ (2,3,7,8-tetra-CDD units) & & 1.258 & 0.0975 \\
\hline
\end{tabular}

${ }^{\text {a }}$ Ball, M., O. Päpke, and A. Lis: Polychlordibenzodioxine und Polychlordibenzofurane in Cigarettenrauch; Beitr. Tabakforsch. Int. 14 (1990) 393-402.

${ }^{b}$ Cigarettes analyzed were the top ten sellers in the German market during the fourth quarter of 1989 and the reported value is the mean of the analytical results. In most cases, the mean represents values from all ten cigarettes; however, in some cases the analyte was not detected, not analyzable, etc. and the mean of all reported values was used.

c Environmental Protection Agency: Health assessment for 2,3,7,8-tetrachlorodibenzo- $p$-dioxin (TCDD) and related compounds; Draft Document EPA/600/P-00/001Ae (May 2000), Chapter 9, Table 9-1, p. 9-35.

d TEQ is the amount of any polychlorodibenzo-p-dioxin or polychlorodibenzofuran expressed as toxic equivalent amounts of 2,3,7,8-tetrachlorodibenzo- $p$-dioxin.

${ }^{\circ}$ ibid. reference c, Table 9-2, p. 9-36.

${ }^{\dagger} \mathrm{CDD}$ is an abbreviation for chlorodibenzo-p-dioxin.

${ }^{9} \mathrm{CDF}$ is an abbreviation for chlorodibenzofuran.

appears to be of little use to researchers attempting to produce a "less hazardous" cigarette or to aid chemical analysts in prioritization of smoke components that require good analytical methods. Although the prohibitions against conducting quantitative risk assessments are legendary $(9$, 
67-69) especially when it involves extrapolation of animal data to humans, it appears that we have reached the proverbial "fork in the road" mentioned in the quotation from Yogi Berra ${ }^{1}$.

\section{COMPARISON OF WORKPLACE EXPOSURE LIMITS WITH SMOKING EXPOSURE}

The next part of our review will read like a US government report because of our repeated use of so many abbreviations/acronyms to shorten the discussion.

There have been at least three comparisons of workplace exposure limits with smoking exposure. These include analyses by toxicologists at RJRT (30), VORHEES et al. (31), and RICKERT and KAISERMAN (25). Both the RJRT toxicologists and VORHEES et al. used American Conference of Governmental Industrial Hygienists (ACGIH) threshold limit values (TLVs) based upon eight-hour time weighted average exposures while RICKERT and KAISERMAN used 15-minute short-term ACGIH exposure limits (STELs) as their safe exposure value. Although it may be argued that the ACGIH STELs are the appropriate standard for comparison, they have the problem of being few in number. To have a comparison among a significant number of smoke toxicants several assumptions must be made, e.g., ACGIH threshold limit values (TLVs) and OSHA or NIOSH permissible exposure levels (PELs) can be substituted for STELs, ACGIH STELs can be compared with TLVs or PELs, and carcinogens that do not have STELs can be assigned the smallest tabled ACGIH STEL value, i.e., $0.0005 \mathrm{mg} / \mathrm{m}^{3}$ that was developed for beryllium. Additionally, ACGIH STELs and TLVs are not enforceable standards and ACGIH has recently been sued for de facto illegally promulgating standards (70).

The RJRT analysis does not address any specific toxicant, but finds that "with very few exceptions, smoke constituents that occur in smoke at levels of $0.5 \mu \mathrm{g} / \mathrm{cig}$ or less present little or no potential for concern". Meanwhile, although VORHEES et al. state that, "The use of TLVs to derive ADIs [note added, acceptable daily intake] is inappropriate because TLVs were developed by the ACGIH specifically for workplace exposures rather than the broad population exposures expected with cigarette smoking", the VORHEES et al. objection to the use of TLVs is incomprehensible because the working population and smoking population are almost indistinguishable. Nevertheless, VORHEES et al. proceed to rank smoke toxicants based upon cigarette yields, number of cigarettes smoked per day, a $10-\mathrm{m}^{3} /$ day inhalation rate for an eight-hour work period, and a body weight of $70 \mathrm{~kg}$.

In the analysis that follows, we have simplified the smoke toxicant ranking process. Instead of ACGIH TLVs, the legally enforceable workplace permissible exposure level, i.e., TWA $_{8}$ in $\mathrm{mg} / \mathrm{m}^{3}$ approved by the US OSHA, is compared with the average daily concentration (ADC) that a pack-a-day smoker breathing $10 \mathrm{~m}^{3}$ of air would obtain during a smoking workday. The calculation is as follows:

\footnotetext{
${ }^{1}$ Yogi Berra was a baseball player for the New York Yankees during the 1940-50s who is famous in the US for his predilection for quotable quotes, e.g., "It's deja vu all over again".
}

$$
\begin{aligned}
\mathrm{ADC}, \mathrm{mg} / \mathrm{m}^{3} & =\frac{\text { per cig yield, } \mathrm{mg} \times 20 \text { cig per day }}{10 \mathrm{~m}^{3} \text { breathing volume }} \\
\% \text { OSHA TWA } & =\frac{\text { ADC }, \mathrm{mg} / \mathrm{m}^{3} \times 100}{\text { OSHA TWA }_{8}}
\end{aligned}
$$

From the results shown in Table 3 , it is perfectly acceptable for an employee to work in an area where the \% of OSHA $\mathrm{TWA}_{8}$ is less than $100 \%$. Of course this assumes that none of the listed compounds has a short-term exposure limit (STEL) that would be exceeded by smoking one cigarette. For the agents listed, none of the STELs is exceeded by smoking one cigarette.

Nicotine is the only smoke component that exceeds the $\mathrm{TWA}_{8}$ permissible concentration. However, acrolein, carbon monoxide, methyl isocyanate, and formic acid concentrations are reasonably high.

Typically, OSHA does not deal with occupational exposure to known carcinogens such as $\mathrm{B} a \mathrm{P}, 2$-aminonaphthalene, etc. other than to note that exposures to these materials should be eliminated either by engineering controls or respiratory protection. However, you will see listed in Table 3, a number of compounds that are considered to be carcinogens, e.g., ethylene oxide, acrylamide, acrylonitrile, benzene. These are exceptions to the general rule.

\section{RANKING OF SMOKE TOXICANTS BY CARCINO- GENIC POTENCY DATABASE VALUES}

There is a general chemophobia among the US population. This fear of chemicals is fueled by presentations or publications in the mass media by advocacy groups who lay the blame for many adverse human health effects based upon the existence of trace synthetic, i.e., manmade, chemicals in our environment. Recent examples of this fear mongering may be found in a May $10^{\text {th }}, 2002$, Public Broadcasting System-aired television special (71) titled "Kids and Chemicals", which poses the question, "Are everyday chemicals harming our kids?" As reported in the June $17^{\text {th }}$, 2002 Chemical and Engineering News editorial (72):

The gist of this one-hour special is that children are unwittingly and constantly exposed to a soup of toxic chemicals-from pesticides to air pollution to lead in paint. Some of these chemicals are known to cause cancer or other health effects in animals, but many chemicals have not been tested for their impact on children.

Additionally, the Rockefeller Family Fund has published a series of full-page advertisements prepared by the Mount Sinai Center for Children's Health \& the Environment (CCHE) (73) during June 2002 in the New York Times and other newspapers.

Beginning in the late 1980s, Bruce Ames, inventor of the Salmonella mutagenicity assay, and several of his colleagues at the University of California at Berkeley began to take a look at the data implicating human health effects from exposure to synthetic chemicals and comparing these chemical exposures with those from naturally-occurring sources. To accomplish this task, Ames and his co-workers developed the Carcinogenic Potency Database (CPDB) (74). A recent review by GOLD et al. (75) summarizes development, analysis, and conclusions reached through the use of the CPDB. Among key points cited in the review are the following: 
Table 3. Comparison of pack-a-day-smoker concentration with OSHA permissible 8-h time weighted average concentration ${ }^{\text {a }}$. Agents in bold have been included in previous lists of MSS toxicants, but no longer appear to be relevant. See footnotes of Table 1 for details.

\begin{tabular}{|c|c|c|c|c|c|}
\hline Agent & CAS no. & $\begin{array}{c}\text { Comp. cig value } \\
\mathrm{mg} / \mathrm{cig}\end{array}$ & $\begin{array}{c}\text { OSHA TWA } \\
\mathrm{mg} / \mathrm{m}^{3}\end{array}$ & $\begin{array}{c}\text { ADC for } 20 \text { cig per day }{ }^{\mathrm{b}} \\
\mathrm{mg} / \mathrm{m}^{3}\end{array}$ & $\begin{array}{c}\% \text { of OSHA } \\
\text { TWA }_{8}\end{array}$ \\
\hline Nicotine & $54-11-5$ & $7.90 \mathrm{E}-01$ & $5.00 \mathrm{E}-01$ & $1.58 \mathrm{E}+00$ & 316 \\
\hline Acrolein & $107-02-8$ & $6.50 \mathrm{E}-02$ & $2.50 E-01$ & $1.30 \mathrm{E}-01$ & 52.0 \\
\hline Carbon monoxide & $630-08-0$ & $1.24 \mathrm{E}+01$ & $5.50 \mathrm{E}+01$ & $2.48 E+01$ & 45.1 \\
\hline Methyl isocyanate & $624-83-9$ & $5.00 \mathrm{E}-03$ & $5.00 \mathrm{E}-02$ & $1.00 E-02$ & 20.0 \\
\hline Formic acid & 64-18-6 & $6.00 \mathrm{E}-01$ & $9.00 \mathrm{E}+00$ & $1.20 \mathrm{E}+00$ & 13.3 \\
\hline Acetic acid & $64-19-7$ & $1.00 E+00$ & $2.50 E+01$ & $2.00 E+00$ & 8.00 \\
\hline Formaldehyde & $50-00-0$ & $2.31 E-02$ & $9.22 \mathrm{E}-01$ & $4.62 \mathrm{E}-02$ & 5.01 \\
\hline Hydroquinone & $123-31-9$ & $4.29 E-02$ & $2.00 \mathrm{E}+00$ & $8.58 \mathrm{E}-02$ & 4.29 \\
\hline 1,3-Butadiene & $106-99-0$ & $4.10 \mathrm{E}-02$ & $2.21 E+00$ & $8.20 \mathrm{E}-02$ & 3.71 \\
\hline Benzene & $71-43-2$ & $4.79 \mathrm{E}-02$ & $3.19 \mathrm{E}+00$ & $9.58 \mathrm{E}-02$ & 3.00 \\
\hline Hydrogen cyanide & 74-90-8 & $1.65 E-01$ & $1.10 E+01$ & $3.30 E-01$ & 3.00 \\
\hline Cadmium & $7440-43-9$ & $6.73 E-05$ & $5.00 E-03$ & $1.35 E-04$ & 2.69 \\
\hline Propionic acid ${ }^{d}$ & 79-09-4 & $3.00 E-01$ & $3.00 E+01$ & $6.00 \mathrm{E}-01$ & 2.00 \\
\hline Nitric oxide & $10102-43-9$ & $2.76 E-01$ & $3.00 E+01$ & $5.52 \mathrm{E}-01$ & 1.84 \\
\hline Acrylamide & 79-06-1 & $2.20 E-03$ & $3.00 E-01$ & $4.40 \mathrm{E}-03$ & 1.47 \\
\hline Nitrobenzene & $98-95-3$ & $2.50 E-02$ & $5.00 \mathrm{E}+00$ & $5.00 \mathrm{E}-02$ & 1.00 \\
\hline Di(2-ethylhexyl) phthalate & $117-81-7$ & $2.00 E-02$ & $5.00 \mathrm{E}+00$ & $4.00 \mathrm{E}-02$ & 0.800 \\
\hline Ethylene oxide & $75-21-8$ & $7.00 \mathrm{E}-03$ & $1.80 \mathrm{E}+00$ & $1.40 \mathrm{E}-02$ & 0.778 \\
\hline Crotonaldehyde & $123-73-9$ & $2.18 E-02$ & $6.00 E+00$ & $4.36 \mathrm{E}-02$ & 0.727 \\
\hline Hydrogen sulfide & $7783-06-4$ & $9.00 \mathrm{E}-02$ & $2.80 E+01$ & $1.80 \mathrm{E}-01$ & 0.643 \\
\hline Acrylonitrile & 107-13-1 & $1.37 E-02$ & $4.34 \mathrm{E}+00$ & $2.74 E-02$ & 0.631 \\
\hline Catechol $^{d}$ & $120-80-9$ & $4.53 E-02$ & $2.00 E+01$ & $9.06 E-02$ & 0.453 \\
\hline Acetaldehyde & $75-07-0$ & $6.40 \mathrm{E}-01$ & $3.60 \mathrm{E}+02$ & $1.28 \mathrm{E}+00$ & 0.356 \\
\hline $\begin{array}{l}\text { DDT } \\
\text { Acetonitrile }\end{array}$ & $\begin{array}{l}50-29-3 \\
75-05-8\end{array}$ & $\begin{array}{l}1.20 \mathrm{E}-03 \\
1.00 \mathrm{E}-01\end{array}$ & $\begin{array}{l}1.00 \mathrm{E}+00 \\
7.00 \mathrm{E}+01\end{array}$ & $\begin{array}{l}2.40 \mathrm{E}-03 \\
2.00 \mathrm{E}-01\end{array}$ & $\begin{array}{l}0.240 \\
0.286\end{array}$ \\
\hline Methylamine & $74-89-5$ & $1.00 \mathrm{E}-02$ & $1.20 \mathrm{E}+01$ & $2.00 E-02$ & 0.167 \\
\hline Lead & $7439-92-1$ & $3.91 \mathrm{E}-05$ & $5.00 \mathrm{E}-02$ & $7.82 \mathrm{E}-05$ & 0.156 \\
\hline Methanol & 67-56-1 & $1.80 \mathrm{E}-01$ & $2.60 E+02$ & $3.60 E-01$ & 0.138 \\
\hline Arsenic & $7440-38-2$ & $5.80 E-06$ & $1.00 \mathrm{E}-02$ & $1.16 \mathrm{E}-05$ & 0.116 \\
\hline Phenol, 4-methyl- & $106-44-5$ & $5.64 E-03$ & $1.00 \mathrm{E}+01$ & $1.13 \mathrm{E}-02$ & 0.113 \\
\hline Phenol & $108-95-2$ & $1.05 E-02$ & $1.90 \mathrm{E}+01$ & $2.10 E-02$ & 0.111 \\
\hline Cyanogen & $460-19-5$ & $1.10 \mathrm{E}-02$ & $2.00 E+01$ & $2.20 \mathrm{E}-02$ & 0.110 \\
\hline Pyridine & 110-86-1 & $7.56 \mathrm{E}-03$ & $1.50 \mathrm{E}+01$ & $1.51 \mathrm{E}-02$ & 0.101 \\
\hline Ammonia & $7664-41-7$ & $1.60 \mathrm{E}-02$ & $3.50 \mathrm{E}+01$ & $3.20 E-02$ & 0.0914 \\
\hline Cobalt & $7440-48-4$ & $3.76 \mathrm{E}-05$ & $1.00 \mathrm{E}-01$ & $7.52 \mathrm{E}-05$ & 0.0752 \\
\hline Sulfur dioxide & $7446-09-5$ & $4.20 E-03$ & $1.30 E+01$ & $8.40 E-03$ & 0.0646 \\
\hline Beryllium & $7440-41-7$ & $5.00 \mathrm{E}-07$ & $2.00 E-03$ & $1.00 E-06$ & 0.0500 \\
\hline Phenol, 3-methyl- & $108-39-4$ & $2.26 \mathrm{E}-03$ & $1.00 E+01$ & $4.52 \mathrm{E}-03$ & 0.0452 \\
\hline 2-Butanone & 78-93-3 & $9.00 \mathrm{E}-02$ & $5.90 \mathrm{E}+02$ & $1.80 \mathrm{E}-01$ & 0.0305 \\
\hline Phenol, 2-methyl- & $95-48-7$ & $3.33 E-03$ & $2.20 \mathrm{E}+01$ & $6.66 \mathrm{E}-03$ & 0.0303 \\
\hline Acetone & 67-64-1 & $2.91 E-01$ & $2.40 E+03$ & $5.82 \mathrm{E}-01$ & 0.0242 \\
\hline Methyl formate & $107-31-3$ & $3.00 E-02$ & $2.50 E+02$ & $6.00 E-02$ & 0.0240 \\
\hline Toluene & $108-88-3$ & $9.04 \mathrm{E}-02$ & $7.54 \mathrm{E}+02$ & $1.81 E-01$ & 0.0234 \\
\hline Furfural & 98-01-1 & $1.40 \mathrm{E}-03$ & $2.00 E+01$ & $2.80 E-03$ & 0.0140 \\
\hline Mercury & $7439-97-6$ & $5.96 \mathrm{E}-06$ & $1.00 \mathrm{E}-01$ & $1.19 E-05$ & 0.0119 \\
\hline Dimethylamine & $124-40-3$ & $1.00 \mathrm{E}-03$ & $1.80 \mathrm{E}+01$ & $2.00 E-03$ & 0.0111 \\
\hline Aniline & 62-53-3 & $6.55 E-04$ & $1.90 \mathrm{E}+01$ & $1.31 \mathrm{E}-03$ & 0.00690 \\
\hline Hydrazine & $302-01-2$ & $4.30 \mathrm{E}-05$ & $1.30 \mathrm{E}+00$ & $8.60 E-05$ & 0.00662 \\
\hline Carbon disulfide & 75-15-0 & $2.00 E-03$ & $6.22 \mathrm{E}+01$ & $4.00 E-03$ & 0.00643 \\
\hline Propane, 2-nitro- & $79-46-9$ & $2.20 \mathrm{E}-03$ & $9.00 \mathrm{E}+01$ & $4.40 \mathrm{E}-03$ & 0.00489 \\
\hline Styrene \{benzene, ethenyl-\} & $100-42-4$ & $7.60 \mathrm{E}-03$ & $4.26 \mathrm{E}+02$ & $1.52 \mathrm{E}-02$ & 0.00357 \\
\hline Resorcinol $^{d}$ & $108-46-3$ & $6.40 \mathrm{E}-04$ & $4.50 \mathrm{E}+01$ & $1.28 \mathrm{E}-03$ & 0.00284 \\
\hline Chromium VI & $1333-82-0$ & $1.32 \mathrm{E}-06$ & $1.00 \mathrm{E}-01$ & $2.64 E-06$ & 0.00264 \\
\hline Vinyl chloride & 75-01-4 & $3.00 E-05$ & $2.56 E+00$ & $6.00 E-05$ & 0.00234 \\
\hline Aniline, 2-methyl- & $95-53-4$ & $2.00 \mathrm{E}-04$ & $2.20 \mathrm{E}+01$ & $4.00 \mathrm{E}-04$ & 0.00182 \\
\hline Naphthalene & $91-20-3$ & $3.42 \mathrm{E}-04$ & $5.00 \mathrm{E}+01$ & $6.84 E-04$ & 0.00137 \\
\hline Selenium & $7782-49-2$ & $1.20 \mathrm{E}-06$ & $2.00 E-01$ & $2.40 E-06$ & 0.00120 \\
\hline Nickel & $7440-02-0$ & $5.58 \mathrm{E}-06$ & $1.00 E+00$ & $1.12 \mathrm{E}-05$ & 0.00112 \\
\hline Chromium & $7440-47-3$ & $4.31 E-06$ & $1.00 \mathrm{E}+00$ & $8.62 E-06$ & 0.000862 \\
\hline
\end{tabular}




\begin{tabular}{l|c|c|c|c|c}
\hline Agent & CAS no. & $\begin{array}{c}\text { Comp. cig value } \\
\mathrm{mg} / \mathrm{cig}\end{array}$ & $\begin{array}{c}\text { OSHA TWA } \\
\mathrm{mg} / \mathrm{m}^{3}\end{array}$ & $\begin{array}{c}\text { ADC for } 20 \text { cig per day } \\
\mathrm{mg}^{\mathrm{b}} / \mathrm{m}^{3}\end{array}$ & $\begin{array}{c}\%^{3} \text { of OSHA } \\
\text { TWA }_{8}\end{array}$ \\
\hline Nitromethane & $75-52-5$ & $6.00 \mathrm{E}-04$ & $2.50 \mathrm{E}+02$ & $1.20 \mathrm{E}-03$ & 0.000480 \\
Aniline, 2,6-dimethyl- & $87-62-7$ & $5.00 \mathrm{E}-05$ & $2.50 \mathrm{E}+01$ & $1.00 \mathrm{E}-04$ & 0.000400 \\
Propylene oxide & $75-56-9$ & $1.00 \mathrm{E}-04$ & $2.40 \mathrm{E}+02$ & $2.00 \mathrm{E}-04$ & 0.000083 \\
\hline
\end{tabular}

a US Department of Health and Human Services: NIOSH pocket guide to chemical hazards (stand-alone HTML version); DHHS (NIOSH) Publication No. 2001-145, August 2001, www.cdc.gov/niosh/npg/npg.html.

${ }^{\mathrm{b}}$ The average daily concentration (ADC) in $\mathrm{mg} / \mathrm{m}^{3}$ is computed by assuming a breathing volume of $10 \mathrm{~m}^{3}$ during the smoking day and smoking 20 cigarettes during that period of time, i.e., $A D C\left(\mathrm{mg} / \mathrm{m}^{3}\right)=$ comparison cig value $(\mathrm{mg} / \mathrm{cig}) \times 20 \mathrm{cig} / \mathrm{day} \div 10 \mathrm{~m}^{3}$ inhaled volume.

c $\%$ of OSHA TWA $8=$ ADC $\left(\mathrm{mg} / \mathrm{m}^{3}\right) \times 100 \div \mathrm{TWA}_{8}\left(\mathrm{mg} / \mathrm{m}^{3}\right)$.

${ }^{d}$ The NIOSH TWA is used because none is established by OSHA.

- Half the chemicals tested in rodent assays are found to be carcinogens; this rate holds whether the chemical is manmade or naturally occurring.

- Among chemicals to which humans are exposed approximately $99.9 \%$ are naturally occurring.

- Because half the natural chemicals tested are positive, human exposures to rodent carcinogens are likely to be ubiquitous.

- In animal cancer tests, the doses administered are at the maximum tolerated dose (MTD). At the MTD a chemical can cause chronic cell killing and cell replacement in a target tissue which is a cancer risk factor itself.

- In high-dose bioassays, cell division increases mutagenesis and therefore carcinogenesis.

- Extrapolation of cancer potency results from MTD studies to real-life exposures is not scientifically supportable.

- Extrapolation of cancer potency results in rodents to humans cannot be validated.

The key value taken from the $\mathrm{CPDB}$ is the $\mathrm{TD}_{50}$. This value is the dose rate in $\mathrm{mg} / \mathrm{kg}$ body $\mathrm{wt} /$ day that will induce tumors in half of test animals that otherwise would have remained tumor-free at zero dose (75). A low value of $\mathrm{TD}_{50}$ indicates a potent carcinogen and a high value indicates a weak one. In the rodent database that we used for our analysis, data may be present either for mice, rats or both. By convention, the lowest $\mathrm{TD}_{50}$ is used for comparison. To compare various exposures, Ames and co-workers use the term \% HERP (Human Exposure to Rodent Potential) that is defined as follows:

$$
\% \text { HERP }=\frac{\text { Actual Dose Rate }, \mathrm{mg} / \mathrm{kg} / \text { day } \times 100}{\mathrm{TD}_{50}, \mathrm{mg} / \mathrm{kg} / \text { day }}
$$

The conventional body weight used in a human comparison is $70 \mathrm{~kg}$. Several important reference points in interpreting $\%$ HERP data are the following: 0.00003 (based on rat $\mathrm{TD}_{50}$ ) and 0.00001 (based on mouse) equate to a risk of one in a million, and the background \% HERP for the average chloroform level in a liter of US tap water is 0.0003 . In Table 4, \% HERP ranking of typical US daily human exposures is shown.

As may be seen by the results shown in Table 4, the one-ina-million risk acceptable to many regulatory agencies is surpassed by every entry in the table except for the rodent carcinogen IQ that originates from eating a fried hamburger. Wine, beer, and coffee drinkers should beware.

Before proceeding with the ranking of MSS toxicants, a word of caution emphasized by GoLD and co-workers (75) needs discussion. Standard practice in regulatory risk assessment for chemicals is to extrapolate high-dose animal data to low-dose human exposure without regards to mechanism. If the mechanism of action were known, it is possible that many of the compounds listed in the CPDB database would not be classified as human carcinogens. For example, $D$-limonene which is listed both in Table 1 as an MSS toxicant and in Table 4 as a rodent carcinogen induces tumors only in male rat kidney tubules with involvement of alpha $_{2 \mathrm{u}}$-globulin nephrotoxicity. This mechanism does not appear to be possible in humans (76). Therefore, there is no convincing evidence that $D$-limonene is a human carcinogen when its mechanism of action is considered.

For this review we have taken the data from the CPDB and applied it when available to the list of MSS toxicants contained in Table 1 . The only assumptions made were that we have a pack-a-day smoker who weighs $70 \mathrm{~kg}$. The results of this analysis are shown in Table 5. As may be seen from the table, only eight of the MSS toxicants for which data are available stand below the one-in-a-million risk category. However, we must keep in mind that just because a compound has a relatively high \% HERP score, e.g., $D$-limonene at 0.008403 , does not make it a human health hazard.

\section{SELECTION OF BEST AVAILABLE CARCINO- GENIC POTENCY VALUES FOR RANKING MSS TOXICANTS}

As mentioned previously, at least two quantitative rankings of MSS toxicants have been made. One ranking by VORHEES et al. (31) in 1997 was in support of the Massachusetts Department of Public Health Tobacco Control Program. The other ranking was part of a year 2000 report to the New Zealand Ministry of Health by FOWLES and BATES (33). Both of these reports contain analyses for carcinogenic effects and non-cancer health effects. Analyses for carcinogenic effects rely on Inhalation Unit Risk Factors in units of $\left(\mathrm{mg} / \mathrm{m}^{3}\right)^{-1}$ as measures of potency. Because some 
Table 4. Possible hazard from daily human exposure of rodent carcinogens; the subscript to the $T_{50}$ values refers to either rat $(R)$ or mouse (M) data. ${ }^{a}$

\begin{tabular}{|c|c|c|c|c|c|}
\hline Human exposure & g/day & Rodent carcinogen & $\mathrm{mg} /$ day $^{\mathrm{b}}$ & $\mathrm{TD}_{50}$ in $\mathrm{mg} / \mathrm{kg}$ & $\%$ HERP \\
\hline Beer & 257 & ethanol & 11479 & $9110_{R}$ & 2.1 \\
\hline Wine & 28.0 & ethanol & 3826 & $9110_{R}$ & 0.5 \\
\hline Home air ${ }^{c}$ & & formaldehyde & 0.598 & $2.19_{R}$ & 0.4 \\
\hline Coffee & 13.3 & caffeic acid & 23.9 & $297_{\mathrm{R}}$ & 0.1 \\
\hline Lettuce & 14.9 & caffeic acid & 7.90 & $297_{\mathrm{R}}$ & 0.04 \\
\hline Black pepper & 0.446 & $D$-limonene & 3.57 & $204_{R}$ & 0.03 \\
\hline Orange juice & 138 & $D$-limonene & 4.28 & $204_{R}$ & 0.03 \\
\hline Safrole in spices & & safrole & 1.2 & $51.3_{\mathrm{M}}$ & 0.03 \\
\hline Apple & 32.0 & caffeic acid & 3.40 & $297_{R}$ & 0.02 \\
\hline Coffee & 13.3 & catechol & 1.33 & $118_{\mathrm{R}}$ & 0.02 \\
\hline Coffee & 13.3 & furfural & 2.09 & $197_{\mathrm{M}}$ & 0.02 \\
\hline Mushroom & 2.55 & hydrazines & & $20300_{M}$ & 0.02 \\
\hline Cinnamon & 21.9 & coumarin & 0.065 & $13.9_{R}$ & 0.007 \\
\hline Coffee & 13.3 & hydroquinone & 0.333 & $82.8_{R}$ & 0.006 \\
\hline Carrot & 12.1 & aniline & 0.624 & $194_{R}$ & 0.005 \\
\hline Celery & 7.95 & caffeic acid & 0.858 & $297_{\mathrm{R}}$ & 0.004 \\
\hline Potato & 54.9 & caffeic acid & 0.867 & $297_{\mathrm{R}}$ & 0.004 \\
\hline White bread & 67.6 & furfural & 0.500 & $197_{M}$ & 0.004 \\
\hline Home air ${ }^{d}$ & & benzene & 0.155 & $77.5_{\mathrm{M}}$ & 0.003 \\
\hline Nutmeg & 0.0274 & $D$-limonene & 0.466 & $204_{R}$ & 0.003 \\
\hline Carrot & 12.1 & caffeic acid & 0.374 & $297_{\mathrm{R}}$ & 0.002 \\
\hline Ethylenethiourea ${ }^{e}$ & & ethylenethiourea & 0.00951 & $7.9_{R}^{n}$ & 0.002 \\
\hline Pear & 3.29 & caffeic acid & 0.240 & $297_{\mathrm{R}}$ & 0.001 \\
\hline Plum & 2.00 & caffeic acid & 0.276 & $297_{R}^{n}$ & 0.001 \\
\hline Brown mustard & 0.0684 & allyl isothiocyanate & 0.0629 & $96_{R}$ & 0.0009 \\
\hline Bacon & 11.5 & $\mathrm{~N}$-nitrosodiethylamine & 0.0000115 & $0.0237_{R}$ & 0.0007 \\
\hline TCDD $^{\dagger}$ & & TCDD & 0.000012 & $0.0000235_{R}$ & 0.0007 \\
\hline Bacon & 11.5 & $N$-nitrosopyrrolidine & 0.000196 & $0.679_{\mathrm{M}}$ & 0.0004 \\
\hline Bacon & 11.5 & $\mathrm{~N}$-nitrosodimethylamine & 0.0000345 & $0.124_{R}$ & 0.0004 \\
\hline Tap water & 1000 & bromodichloromethane & 0.013 & $47.7_{\mathrm{M}}$ & 0.0004 \\
\hline Tap water & 1000 & chloroform & 0.017 & $90.3_{\mathrm{M}}$ & 0.0003 \\
\hline Beer & 257 & furfural & 0.0399 & $197_{M}$ & 0.0003 \\
\hline $\mathrm{PCBs}^{9}$ & & PCBs & 0.000098 & $1.74_{R}$ & 0.00008 \\
\hline Toast & 67.6 & ethyl carbamate \{urethane\} & 0.000811 & $16.9_{\mathrm{M}}^{\mathrm{n}}$ & 0.00007 \\
\hline Hamburger & 85 & PhIP & 0.000176 & $4.29_{\mathrm{R}}$ & 0.00006 \\
\hline Hamburger & 85 & MelQx & 0.0000381 & $1.99_{R}$ & 0.00003 \\
\hline Beer & 257 & ethyl carbamate \{urethane\} & 0.000115 & $16.9_{\mathrm{M}}$ & 0.00001 \\
\hline Hamburger & 85 & $\mathrm{IQ}$ & 0.00000638 & $1.89_{R}$ & 0.000005 \\
\hline
\end{tabular}

${ }^{a}$ Gold, L.S., T.H. Slone and B.N. Ames: Overview of analyses of the carcinogenic potency database; in: Handbook of carcinogenic potency and genotoxicity databases, edited by L.S. Gold and E. Zeiger, CRC Press, Boca Raton, FL, 1997, at http://potency.berkeley.edu/herp.html, downloaded from the Internet on March 24, 2002.

${ }^{\mathrm{b}}$ Calculations assume a 70-kg person.

${ }^{c}$ Value assumes a 14-h exposure per day.

d Assumes a 14-h daily exposure in a conventional home.

e Daily US average for 1990.

t 2,3,7,8-Tetrachlorodibenzo-p-dioxin daily US average for 1994 .

${ }^{9}$ Daily US average over the period 1984-1986.

of these unit risk factors change frequently and different sources have different values for the same chemical compound, we decided to compile a list of these factors from available sources. Once compiled, a selection was made from available values to use in further analysis. Inhalation unit risk values available for MSS toxicants shown in Table 1 are presented in Table 6.
The values are taken from four major resources including the California EPA Office of Environmental Health Hazard Assessment, the US EPA Integrated Risk Information System (IRIS), the US Department of Energy, Office of Environmental Management, Oak Ridge Operations Office: Risk assessment information system (RAIS), and the US EPA: National-scale toxics assessment (NATA) for 1996. 
Table 5. Comparison of pack-a-day smoker intake with HERP Index ${ }^{\text {a }}$. Agents in bold have been included in previous lists of cigarette MSS toxicants, but no longer appear to be relevant. See footnotes of Table 1 for details. A \% HERP ranking of $0.00003 \%$ (based on a rat $\mathrm{TD}_{50}$ ) or $0.00001 \%$ (based on a mouse $\mathrm{TD}_{50}$ ) equates to a risk of 1 in a million.

\begin{tabular}{|c|c|c|c|c|c|c|c|}
\hline Agent & CAS no. & $\begin{array}{l}\text { Comp. cig value } \\
\mu \mathrm{g} / \mathrm{cig}\end{array}$ & $\begin{array}{c}\mathrm{TD}_{50}{ }^{\mathrm{b}} \\
\mathrm{mg} / \mathrm{kg} / \text { day }\end{array}$ & $\begin{array}{l}\text { Species } \\
\text { rat or mouse }\end{array}$ & $\begin{array}{l}\text { Intake }^{c} \\
\text { mg/day }\end{array}$ & $\begin{array}{l}\text { Intake/wt } \\
\text { mg/kg/day }\end{array}$ & $\%$ HERP \\
\hline Formaldehyde & $50-00-0$ & $2.31 E+01$ & $2.19 \mathrm{E}+00$ & $\mathrm{R}$ & $4.62 \mathrm{E}-01$ & $6.60 E-03$ & 0.301 \\
\hline Crotonaldehyde & $123-73-9$ & $2.18 \mathrm{E}+01$ & $4.20 \mathrm{E}+00$ & $\mathrm{R}$ & $4.36 E-01$ & $6.23 E-03$ & 0.148 \\
\hline Acetaldehyde & $75-07-0$ & $6.40 \mathrm{E}+02$ & $1.53 \mathrm{E}+02$ & $\mathrm{R}$ & $1.28 E+01$ & $1.83 \mathrm{E}-01$ & 0.120 \\
\hline 1,3-Butadiene & $106-99-0$ & 4.10 E+01 & $1.39 \mathrm{E}+01$ & M & $8.20 \mathrm{E}-01$ & $1.17 \mathrm{E}-02$ & 0.0843 \\
\hline Ethyl carbamate \{urethane\} & $51-79-6$ & $3.80 \mathrm{E}+01$ & $1.69 \mathrm{E}+01$ & M & $7.60 \mathrm{E}-01$ & $1.09 \mathrm{E}-02$ & 0.0642 \\
\hline Isoprene & $78-79-5$ & 4.47 E+02 & $2.74 \mathrm{E}+02$ & M & $8.94 E+00$ & $1.28 \mathrm{E}-01$ & 0.0466 \\
\hline Nitrobenzene & $98-95-3$ & $2.50 \mathrm{E}+01$ & $2.55 E+01$ & $\mathrm{R}$ & $5.00 \mathrm{E}-01$ & $7.14 E-03$ & 0.0280 \\
\hline $\begin{array}{l}\text { 4-(N-Methylnitrosamino)-1-(3- } \\
\text { pyridinyl)-1-butanone }\end{array}$ & $64091-91-4$ & $9.70 \mathrm{E}-02$ & $9.99 \mathrm{E}-02$ & $\mathrm{R}$ & $1.94 E-03$ & $2.77 \mathrm{E}-05$ & 0.0277 \\
\hline Benzene & $71-43-2$ & $4.79 E+01$ & $7.75 E+01$ & M & $9.58 E-01$ & $1.37 E-02$ & 0.0177 \\
\hline Catechol & $120-80-9$ & $4.53 E+01$ & $8.47 \mathrm{E}+01$ & $\mathrm{R}$ & $9.06 \mathrm{E}-01$ & $1.29 \mathrm{E}-02$ & 0.0153 \\
\hline Hydroquinone & $123-31-9$ & $4.29 \mathrm{E}+01$ & $8.28 \mathrm{E}+01$ & $\mathrm{R}$ & $8.58 \mathrm{E}-01$ & $1.23 E-02$ & 0.0148 \\
\hline Acrylamide & $79-06-1$ & $2.20 \mathrm{E}+00$ & $6.15 \mathrm{E}+00$ & $\mathrm{R}$ & $4.40 E-02$ & $6.29 E-04$ & 0.0102 \\
\hline Ethylene oxide & $75-21-8$ & $7.00 \mathrm{E}+00$ & $2.13 E+01$ & $\mathrm{R}$ & $1.40 \mathrm{E}-01$ & $2.00 \mathrm{E}-03$ & 0.00939 \\
\hline Styrene \{benzene, ethenyl-\} & $100-42-4$ & $7.60 \mathrm{E}+00$ & $2.33 E+01$ & $\mathrm{R}$ & $1.52 \mathrm{E}-01$ & $2.17 \mathrm{E}-03$ & 0.00932 \\
\hline$D$-Limonene & 5989-27-5 & $6.00 \mathrm{E}+01$ & $2.04 E+02$ & $\mathrm{R}$ & $1.20 \mathrm{E}+00$ & $1.71 \mathrm{E}-02$ & 0.00840 \\
\hline N-Nitrosoethylmethylamine & $10595-95-6$ & $1.30 \mathrm{E}-02$ & $5.03 \mathrm{E}-02$ & $\mathrm{R}$ & $2.60 E-04$ & $3.71 E-06$ & 0.00738 \\
\hline N-Ntrosodimethylamine & $62-75-9$ & $1.91 \mathrm{E}-02$ & $9.59 \mathrm{E}-02$ & $\mathrm{R}$ & $3.82 E-04$ & $5.46 \mathrm{E}-06$ & 0.00569 \\
\hline$N$-Nitrosopiperidine & $100-75-4$ & $2.31 \mathrm{E}-01$ & $1.30 \mathrm{E}+00$ & M & $4.62 \mathrm{E}-03$ & $6.60 \mathrm{E}-05$ & 0.00508 \\
\hline Hydrazine & $302-01-2$ & $4.30 \mathrm{E}-02$ & $3.09 \mathrm{E}-01$ & $\mathrm{R}$ & $8.60 E-04$ & $1.23 E-05$ & 0.00398 \\
\hline N-Nitrosodiethylamine & $55-18-5$ & $2.80 \mathrm{E}-03$ & $2.65 E-02$ & $\mathrm{R}$ & $5.60 \mathrm{E}-05$ & $8.00 \mathrm{E}-07$ & 0.00302 \\
\hline DDT & $50-29-3$ & $1.20 \mathrm{E}+00$ & $1.28 \mathrm{E}+01$ & M & $2.40 \mathrm{E}-02$ & $3.43 \mathrm{E}-04$ & 0.00268 \\
\hline$N$-Nitrosodi- $n$-butylamine & $924-16-3$ & $3.00 \mathrm{E}-02$ & $6.91 \mathrm{E}-01$ & $\mathrm{R}$ & $6.00 \mathrm{E}-04$ & $8.57 \mathrm{E}-06$ & 0.00124 \\
\hline Di(2-ethylhexyl) phthalate & $117-81-7$ & $2.00 \mathrm{E}+01$ & $6.25 E+02$ & $\mathrm{R}$ & $4.00 E-01$ & $5.71 \mathrm{E}-03$ & 0.000914 \\
\hline DDE & $72-55-9$ & $3.70 \mathrm{E}-01$ & $1.25 E+01$ & $M$ & $7.40 \mathrm{E}-03$ & $1.06 \mathrm{E}-04$ & 0.000846 \\
\hline Toluene & $108-88-3$ & $9.04 \mathrm{E}+01$ & $3.06 \mathrm{E}+03$ & $\mathrm{R}$ & $1.81 E+00$ & $2.58 E-02$ & 0.000844 \\
\hline $\begin{array}{l}\text { 2,3,7,8-Tetrachlorodibenzo-p- } \\
\quad{\text { dioxin }(T E Q)^{\mathrm{e}}}^{\mathrm{e}}\end{array}$ & $1746-01-6$ & $1.26 \mathrm{E}-06$ & $4.57 E-05$ & $\mathrm{R}$ & $2.52 \mathrm{E}-08$ & $3.60 E-10$ & 0.000788 \\
\hline Acetamide & $60-35-5$ & $3.97 \mathrm{E}+00$ & $1.80 \mathrm{E}+02$ & $\mathrm{R}$ & $7.94 \mathrm{E}-02$ & $1.13 \mathrm{E}-03$ & 0.000630 \\
\hline$N$-Nitrosopyrrolidine & $930-55-2$ & $1.40 \mathrm{E}-02$ & $6.79 \mathrm{E}-01$ & M & $2.80 \mathrm{E}-04$ & $4.00 \mathrm{E}-06$ & 0.000589 \\
\hline $\operatorname{PhIP}(\mathrm{HCl})^{f}$ & $105650-23-5$ & $2.30 \mathrm{E}-02$ & $1.91 \mathrm{E}+00$ & $\mathrm{R}$ & $4.60 \mathrm{E}-04$ & $6.57 E-06$ & 0.000344 \\
\hline Vinyl acetate & $108-05-4$ & $4.00 \mathrm{E}+00$ & $3.41 E+02$ & $\mathrm{R}$ & $8.00 \mathrm{E}-02$ & $1.14 E-03$ & 0.000335 \\
\hline Caffeic acid & $331-39-5$ & $3.00 \mathrm{E}+00$ & $2.97 \mathrm{E}+02$ & $\mathrm{R}$ & $6.00 \mathrm{E}-02$ & $8.57 \mathrm{E}-04$ & 0.000289 \\
\hline Furfural & $98-01-1$ & $1.40 E+00$ & $1.97 \mathrm{E}+02$ & $M$ & $2.80 E-02$ & $4.00 \mathrm{E}-04$ & 0.000203 \\
\hline Carbazole & $86-74-8$ & $1.00 \mathrm{E}+00$ & $1.64 \mathrm{E}+02$ & $M$ & $2.00 E-02$ & $2.86 \mathrm{E}-04$ & 0.000174 \\
\hline Benzo[a]pyrene & $50-32-8$ & $5.70 \mathrm{E}-03$ & $9.56 \mathrm{E}-01$ & $\mathrm{R}$ & $1.14 E-04$ & $1.63 E-06$ & 0.000170 \\
\hline$N$-Nitrosodi- $n$-propylamine & 621-64-7 & $1.00 \mathrm{E}-03$ & $1.86 \mathrm{E}-01$ & $\mathrm{R}$ & $2.00 \mathrm{E}-05$ & $2.86 \mathrm{E}-07$ & 0.000154 \\
\hline $\mathrm{A} \propto \mathrm{C}$ & $26148-68-5$ & $2.60 \mathrm{E}-01$ & $4.98 \mathrm{E}+01$ & $\mathrm{R}$ & $5.20 \mathrm{E}-03$ & $7.43 \mathrm{E}-05$ & 0.000149 \\
\hline Aniline, 2-methyl- $(\mathrm{HCl})^{g}$ & $95-53-4$ & $2.00 \mathrm{E}-01$ & $4.36 \mathrm{E}+01$ & $\mathrm{R}$ & $4.00 E-03$ & $5.71 \mathrm{E}-05$ & 0.000131 \\
\hline Ethylenethiourea & $96-45-7$ & $2.70 \mathrm{E}-02$ & $7.90 \mathrm{E}+00$ & $\mathrm{R}$ & $5.40 \mathrm{E}-04$ & $7.71 \mathrm{E}-06$ & 0.000098 \\
\hline Aniline $^{f}$ & $62-53-3$ & $6.55 \mathrm{E}-01$ & $2.69 \mathrm{E}+02$ & $\mathrm{R}$ & $1.31 \mathrm{E}-02$ & $1.87 \mathrm{E}-04$ & 0.000070 \\
\hline Naphthalene & 91-20-3 & $3.42 \mathrm{E}-01$ & $1.63 \mathrm{E}+02$ & M & $6.84 E-03$ & $9.77 \mathrm{E}-05$ & 0.000060 \\
\hline N'-Nitrosoanabasine & $37620-20-5$ & $2.31 \mathrm{E}-02$ & $1.19 \mathrm{E}+01$ & $\mathrm{R}$ & $4.62 \mathrm{E}-04$ & $6.60 \mathrm{E}-06$ & 0.000055 \\
\hline Biphenyl, 4-amino- & $92-67-1$ & $4.00 \mathrm{E}-03$ & $2.10 \mathrm{E}+00$ & M & $8.00 \mathrm{E}-05$ & $1.14 \mathrm{E}-06$ & 0.000054 \\
\hline MeA $\propto \mathrm{C}(\text { acetate })^{\mathrm{h}}$ & $68006-83-7$ & $3.70 \mathrm{E}-02$ & $2.22 \mathrm{E}+01$ & $\mathrm{R}$ & $7.40 \mathrm{E}-04$ & $1.06 \mathrm{E}-05$ & 0.000048 \\
\hline Vinyl chloride & 75-01-4 & $3.00 \mathrm{E}-02$ & $1.91 \mathrm{E}+01$ & $\mathrm{R}$ & $6.00 \mathrm{E}-04$ & $8.57 \mathrm{E}-06$ & 0.000045 \\
\hline N-Nitrosodiethanolamine & $1116-54-7$ & $4.30 \mathrm{E}-03$ & $3.17 \mathrm{E}+00$ & $\mathrm{R}$ & $8.60 \mathrm{E}-05$ & $1.23 E-06$ & 0.000039 \\
\hline Propylene oxide & $75-56-9$ & $1.00 \mathrm{E}-01$ & $7.44 \mathrm{E}+01$ & $\mathrm{R}$ & $2.00 E-03$ & $2.86 \mathrm{E}-05$ & 0.000038 \\
\hline Trp-P-1 (acetate) ${ }^{f}$ & $62450-06-0$ & $5.00 \mathrm{E}-04$ & $5.75 \mathrm{E}-01$ & $\mathrm{R}$ & $1.00 E-05$ & $1.43 \mathrm{E}-07$ & 0.000025 \\
\hline $\mathrm{IQ}$ & $76180-96-6$ & $3.00 \mathrm{E}-04$ & $8.12 \mathrm{E}-01$ & $\mathrm{R}$ & $6.00 \mathrm{E}-06$ & $8.57 E-08$ & 0.000011 \\
\hline Naphthalene, 2-amino- & $91-59-8$ & $1.11 \mathrm{E}-02$ & $3.67 \mathrm{E}+01$ & M & $2.22 \mathrm{E}-04$ & $3.17 E-06$ & 0.000009 \\
\hline Glu-P-1 & $67730-11-4$ & $8.90 \mathrm{E}-04$ & $4.69 \mathrm{E}+00$ & $\mathrm{R}$ & $1.78 \mathrm{E}-05$ & $2.54 \mathrm{E}-07$ & 0.000005 \\
\hline Trp-P-2 (acetate) ${ }^{f}$ & $62450-07-1$ & $1.10 \mathrm{E}-03$ & $6.66 \mathrm{E}+00$ & $\mathrm{R}$ & $2.20 \mathrm{E}-05$ & $3.14 \mathrm{E}-07$ & 0.000005 \\
\hline
\end{tabular}




\begin{tabular}{l|c|c|c|c|c|c|c}
\hline Agent & CAS no. & $\begin{array}{c}\text { Comp. cig value } \\
\mu \mathrm{g} / \mathrm{cig}\end{array}$ & $\begin{array}{c}\mathrm{TD}_{50}^{\mathrm{b}} \\
\mathrm{mg} / \mathrm{kg} / \mathrm{day}\end{array}$ & $\begin{array}{c}\text { Species } \\
\text { rat or mouse }\end{array}$ & $\begin{array}{l}\text { Intake }^{\mathrm{c}} \\
\mathrm{mg} / \mathrm{day}\end{array}$ & $\begin{array}{l}\text { Intake/wt }^{\mathrm{d}} \\
\mathrm{mg} / \mathrm{kg} / \mathrm{day}\end{array}$ & $\begin{array}{c}\% \mathrm{HERP} \\
\%\end{array}$ \\
\hline Dibenz[a,h]anthracene & $53-70-3$ & $4.00 \mathrm{E}-04$ & $5.88 \mathrm{E}+00$ & $\mathrm{M}$ & $8.00 \mathrm{E}-06$ & $1.14 \mathrm{E}-07$ & 0.000002 \\
MelQ & $77094-11-2$ & $7.50 \mathrm{E}-04$ & $1.23 \mathrm{E}+01$ & $\mathrm{M}$ & $1.50 \mathrm{E}-05$ & $2.14 \mathrm{E}-07$ & 0.000002 \\
Glu-P-2 & $67730-10-3$ & $8.80 \mathrm{E}-04$ & $1.60 \mathrm{E}+01$ & $\mathrm{M}$ & $1.76 \mathrm{E}-05$ & $2.51 \mathrm{E}-07$ & 0.000002 \\
\hline
\end{tabular}

${ }^{a}$ Gold, L.S., T.H. Slone, and B.N. Ames: Overview of analyses of the carcinogenic potency database; in: Handbook of Carcinogenic Potency and Genotoxicity Databases, edited by L.S. Gold and E. Seiger, CRC Press, Boca Raton, FL, 1997, pp. 1-605. Accessed on the Internet, http://potency.berkley.edu/herp.html, March 24, 2002.

${ }^{\mathrm{b}}$ Gold, L.S. T.H. Slone, and B.N. Ames: Chapter 3. Summary of carcinogenic potency database by chemical; in: Handbook of Carcinogenic Potency and Genotoxicity Databases, edited by L.S. Gold and E. Seiger, CRC Press, Boca Raton, FL, 1997, pp. 621-660. Accessed on the Internet, http://potency.berkeley.edu/txt/crc.chapter3.html, March 24, 2002.

${ }^{\circ}$ Assumes a pack-a-day smoker, i.e., 20 cig/day.

${ }^{\mathrm{d}}$ Assumes that a smoker weighs $70 \mathrm{~kg}$.

${ }^{\mathrm{e}}$ In the reference for polychlorodibenzodioxins and polychlorodibenzofurans [Beitr. Tabakforsch. Int. 14 (1990) 393-402] the authors report that the most toxicologically potent isomer of these materials, i.e., 2,3,7,8-tetrachlorodibenzo-p-dioxin (2,3,7,8-TCDD) is not detected in cigarette mainstream smoke. However, for toxicological comparisons, it is common practice to convert all of the isomers present to their 2,3,7,8-TCDD equivalents. The chlorinated dioxins and benzofurans reported in the article were converted to the toxic equivalents of 2,3,7,8TCDD with toxic equivalency factors taken from the following source: U.S. EPA: Exposure and human health reassessment of 2,3,7,8tetracholorodibenzo- $p$-dioxin (TCDD) and related compounds, Part II. Health assessment for 2,3,7,8-tetrachlorodibenzo- $p$-dioxin (TCDD) and related compounds, Chapt. 9, Toxicity equivalence factors (TEF) for dioxin and related compounds, EPA/600/P-00/001Bb (2000), draft final report, Table 9-1, 9-35.

${ }^{f} \mathrm{TD}_{50}$ value is for the $\mathrm{HCl}$ salt and has not been adjusted to the free base value.

${ }^{9} \mathrm{TD}_{50}$ value is for the $\mathrm{HCl}$ salt and has been adjusted to the free base value.

${ }^{\mathrm{h}} \mathrm{TD}_{50}$ value is for the acetate and has not been adjusted to the free compound.

All of these databases are downloadable from the Internet and the websites are noted in the footnotes of Table 6 . When multiple inhalation unit risk values were available, we selected in the following order: the highest value from either the IRIS or RAIS databases, followed by the California EPA value, and followed by the NATA data as our "selected unit risk value" for further cancer risk estimation. Most of the unit risk factors are similar across sources. The cancer risk calculation for ${ }^{210} \mathrm{Po}$ is different because it is based upon radioactivity emissions and is treated separately.

\section{CALCULATION OF INCREMENTAL CANCER LIFETIME RISK FOR EXPOSURE TO MSS TOXICANTS}

The calculation of incremental cancer lifetime risk for exposure to MSS toxicants follows the procedure and assumptions made by VORHEES et al. (31). For each toxicant in Table 1 an average daily concentration (ADC) is calculated according to the following formula:

$$
\mathrm{ADC}, \mathrm{mg} / \mathrm{m}^{3}=\frac{\text { Yield per cig, } \mathrm{mg} \times \text { Number cig smoked } / \text { day }}{\text { Volume of air breathed } / \text { day }}
$$

where the volume of air breathed per day is assumed to be $20 \mathrm{~m}^{3}$.

The incremental lifetime cancer risk is then calculated as follows:

$$
\text { Incremental Lifetime Cancer Risk }=\mathrm{ADC}_{\text {life }} \times \mathrm{URF}
$$

where:

$$
\begin{array}{ll}
\mathrm{ADC}_{\text {life }} & =\text { Lifetime Average Daily Concentration }\left(\mathrm{mg} / \mathrm{m}^{3}\right) \\
\mathrm{URF} & =\text { Unit Risk Factor }\left(\mathrm{mg} / \mathrm{m}^{3}\right)^{-1}
\end{array}
$$

The lifetime ADC is estimated by adjusting the daily ADC according to the number of years of smoking (assumed to be 35 years) and the average lifetime (assumed to be 70 years). The equation relating the daily $\mathrm{ADC}$ to $\mathrm{ADC}_{\text {life }}$ is as follows:

$$
\mathrm{ADC}_{\text {life }}=\frac{\mathrm{ADC} \times \text { Number of years smoking }}{\text { Average lifetime }}
$$

We have assumed 35 years of smoking rather than the 30 years used by VORHEES et al.

The results of incremental lifetime cancer risk calculations employing yield data from Table 1 and assuming that the person is a pack-a-day smoker are shown in Table 7.

As mentioned earlier, excess incremental lifetime cancer risk (ILCR) for exposure to ${ }^{210} \mathrm{Po}$ is calculated differently than the other MSS toxicants in Table 7. The calculation is as follows:

$$
\begin{aligned}
\mathrm{ILCR}_{\mathrm{Po}-210}= & \mathrm{pCi} / \mathrm{cig} \times \text { Cig smoked } / \text { day } \times \text { Days } / \text { year } \\
& \times \text { Number of smoking years } \times \text { risk } / \mathrm{pCi} \\
= & 1.60 \mathrm{E}-02 \mathrm{pCi} / \mathrm{cig} \times 20 \mathrm{cig} / \mathrm{day} \times 365 \mathrm{day} / \mathrm{yr} \\
& \times 35 \text { smoking years } \times 1.08 \mathrm{E}-08 \mathrm{risk} / \mathrm{pCi} \\
= & 4.42 \mathrm{E}-05
\end{aligned}
$$

Thus, the calculated excess lifetime cancer risk for a packa-day smoker of $1 \mathrm{R} 4 \mathrm{~F}$ cigarettes is estimated to be greater than one in a million. However the risk from ${ }^{210} \mathrm{Po}$ does not appear to be very large. In their comparison of lung cancer incidence in uranium miners exposed to ${ }^{210} \mathrm{Po}$ vs. cigarette smokers exposed to MSS ${ }^{210}$ Po, HARLEY et al. (77) questioned the significance of ${ }^{210} \mathrm{Po}$ in tobacco-induced lung cancer. Their conclusion has often been quoted (7). 
Table 6. Cancer potency values for some toxicants in cigarette mainstream smoke. Agents in bold have been included in previous lists of cigarette MSS toxicants, but no longer appear to be relevant. See footnotes of Table 1 for details.

\begin{tabular}{|c|c|c|c|c|c|c|}
\hline Agent & CAS no. & $\begin{array}{c}\text { Cal. EPA } \\
\text { inhal. unit risk a } \\
\left(\mathrm{mg} / \mathrm{m}^{3}\right)^{-1}\end{array}$ & \begin{tabular}{|c|} 
US EPA \\
$\begin{array}{c}\text { inhal. unit risk } \\
\left(\mathrm{mg} / \mathrm{m}^{3}\right)^{-1}\end{array}$ \\
\end{tabular} & \begin{tabular}{|c|} 
NATA 1996 \\
$\begin{array}{c}\text { inhal. unit risk } \\
\left(\mathrm{mg} / \mathrm{m}^{3}\right)^{-1}\end{array}$
\end{tabular} & \begin{tabular}{|c} 
ORNL \\
$\begin{array}{c}\text { inhal. unit risk e } \\
\left(\mathrm{mg} / \mathrm{m}^{3}\right)^{-1}\end{array}$
\end{tabular} & $\begin{array}{c}\text { Selected } \\
\text { inhal. unit risk } \\
\left(\mathrm{mg} / \mathrm{m}^{3}\right)^{-1}\end{array}$ \\
\hline N-Nitrosodiethylamine & $55-18-5$ & $1.00 \mathrm{E}+01$ & & & $4.30 \mathrm{E}+01$ & $4.30 \mathrm{E}+01$ \\
\hline N-Nitrosodimethylamine & $62-75-9$ & $4.60 \mathrm{E}+00$ & & & $1.40 \mathrm{E}+01$ & $1.40 \mathrm{E}+01$ \\
\hline Chromium VI & $1333-82-0$ & $1.50 \mathrm{E}+02$ & $1.20 \mathrm{E}+01$ & & $1.20 \mathrm{E}+01$ & $1.20 \mathrm{E}+01$ \\
\hline Dibenzo[b,def]chrysene & 189-64-0 & $1.10 \mathrm{E}+01$ & & & & $1.10 \mathrm{E}+01$ \\
\hline $\begin{array}{l}\text { Dibenzo[def,p]chrysene } \\
\text { Trp-P-1 }\end{array}$ & $\begin{array}{r}191-30-0 \\
62450-06-0\end{array}$ & $\begin{array}{l}1.10 \mathrm{E}+01 \\
7.40 \mathrm{E}+00\end{array}$ & & & & $\begin{array}{l}1.10 \mathrm{E}+01 \\
7.40 \mathrm{E}+00\end{array}$ \\
\hline$N$-Nitrosoethylmethylamine & $10595-95-6$ & $6.30 \mathrm{E}+00$ & & & & $6.30 \mathrm{E}+00$ \\
\hline Biphenyl, 4-amino- & $92-67-1$ & $6.00 \mathrm{E}+00$ & & & & $6.00 \mathrm{E}+00$ \\
\hline Hydrazine & $302-01-2$ & $4.90 \mathrm{E}+00$ & $4.90 \mathrm{E}+00$ & & $4.90 \mathrm{E}+00$ & $4.90 \mathrm{E}+00$ \\
\hline Hydrazine, 1,1-dimethyl- & 57-14-7 & & & & $4.90 \mathrm{E}+00$ & $4.90 \mathrm{E}+00$ \\
\hline Arsenic & $7440-38-2$ & $3.30 \mathrm{E}+00$ & $4.30 \mathrm{E}+00$ & & $4.30 \mathrm{E}+00$ & $4.30 \mathrm{E}+00$ \\
\hline Quinoline & $91-22-5$ & & & $3.40 \mathrm{E}+00$ & & $3.40 \mathrm{E}+00$ \\
\hline N-Nitrosopiperidine & $100-75-4$ & $2.70 \mathrm{E}+00$ & & & & $2.70 \mathrm{E}+00$ \\
\hline Propane, 2-nitro- & $79-46-9$ & & & & $2.70 \mathrm{E}+00$ & $2.70 \mathrm{E}+00$ \\
\hline Beryllium & $7440-41-7$ & $2.40 \mathrm{E}+00$ & $2.40 \mathrm{E}+00$ & & $2.40 \mathrm{E}+00$ & $2.40 \mathrm{E}+00$ \\
\hline$N$-Nitrosodi- $n$-propylamine & 621-64-7 & $2.00 \mathrm{E}+00$ & & & & $2.00 E+00$ \\
\hline Cadmium & $7440-43-9$ & $4.20 \mathrm{E}+00$ & $1.80 \mathrm{E}+00$ & & $1.80 \mathrm{E}+00$ & $1.80 \mathrm{E}+00$ \\
\hline$N$-Nitrosodi- $n$-butylamine & $924-16-3$ & $3.10 \mathrm{E}+00$ & & & $1.60 \mathrm{E}+00$ & $1.60 \mathrm{E}+00$ \\
\hline Glu-P-1 & $67730-11-4$ & $1.40 \mathrm{E}+00$ & & & & $1.40 \mathrm{E}+00$ \\
\hline Acrylamide & 79-06-1 & $1.30 \mathrm{E}+00$ & & & $1.30 \mathrm{E}+00$ & $1.30 \mathrm{E}+00$ \\
\hline $7 H$-Dibenzo $[c, g]$ carbazole & 194-59-2 & $1.10 \mathrm{E}+00$ & & & & $1.10 \mathrm{E}+00$ \\
\hline Chrysene, 5-methyl- & $3697-24-3$ & $1.10 \mathrm{E}+00$ & & & & $1.10 \mathrm{E}+00$ \\
\hline Naphtho[1,2,3,4-def]chrysene & $192-65-4$ & $1.10 \mathrm{E}+00$ & & & & $1.10 \mathrm{E}+00$ \\
\hline Trp-P-2 & $62450-07-1$ & $9.10 \mathrm{E}-01$ & & & & $9.10 \mathrm{E}-01$ \\
\hline Benzo[a]pyrene & $50-32-8$ & $1.10 \mathrm{E}+00$ & & & $8.80 \mathrm{E}-01$ & $8.80 \mathrm{E}-01$ \\
\hline Dibenz $[a, h]$ anthracene & 53-70-3 & $1.20 E+00$ & & & $8.80 \mathrm{E}-01$ & $8.80 E-01$ \\
\hline N-Nitrosodiethanolamine & $1116-54-7$ & $8.00 \mathrm{E}-01$ & & & & $8.00 \mathrm{E}-01$ \\
\hline$N$-Nitrosopyrrolidine & $930-55-2$ & $6.00 \mathrm{E}-01$ & & & $6.10 \mathrm{E}-01$ & $6.10 \mathrm{E}-01$ \\
\hline Naphthalene, 2-amino- & 91-59-8 & $5.14 \mathrm{E}-01$ & & & & $5.14 \mathrm{E}-01$ \\
\hline Glu-P-2 & $67730-10-3$ & $4.00 \mathrm{E}-01$ & & & & $4.00 \mathrm{E}-01$ \\
\hline IQ & $76180-96-6$ & $4.00 \mathrm{E}-01$ & & & & $4.00 \mathrm{E}-01$ \\
\hline N'-Nitrosonornicotine & $16543-55-8$ & $4.00 \mathrm{E}-01$ & & & & $4.00 E-01$ \\
\hline $\mathrm{MeA} \alpha \mathrm{C}$ & $68006-83-7$ & $3.40 \mathrm{E}-01$ & & & & $3.40 \mathrm{E}-01$ \\
\hline Ethyl carbamate \{urethane\} & 51-79-6 & $2.90 \mathrm{E}-01$ & & & & $2.90 \mathrm{E}-01$ \\
\hline 1,3-Butadiene & $106-99-0$ & $1.70 \mathrm{E}-01$ & $2.80 \mathrm{E}-01$ & & $2.80 \mathrm{E}-01$ & $2.80 E-01$ \\
\hline Nickel & $7440-02-0$ & $2.60 \mathrm{E}-01$ & $2.40 \mathrm{E}-01$ & & $2.40 \mathrm{E}-01$ & $2.40 \mathrm{E}-01$ \\
\hline $\mathrm{A} \propto \mathrm{C}$ & $26148-68-5$ & $1.14 \mathrm{E}-01$ & & & & $1.14 \mathrm{E}-01$ \\
\hline Benzo[]fluoranthene & 205-82-3 & $1.10 \mathrm{E}-01$ & & & & $1.10 \mathrm{E}-01$ \\
\hline Dibenz $[a, h]$ acridine & $226-36-8$ & $1.10 \mathrm{E}-01$ & & & & $1.10 \mathrm{E}-01$ \\
\hline Dibenz $[a, j]$ acridine & $224-42-0$ & $1.10 \mathrm{E}-01$ & & & & $1.10 \mathrm{E}-01$ \\
\hline Ethylene oxide & $75-21-8$ & $8.80 \mathrm{E}-02$ & & & $1.00 \mathrm{E}-01$ & $1.00 \mathrm{E}-01$ \\
\hline DDT & $50-29-3$ & & & & $9.70 \mathrm{E}-02$ & $9.70 E-02$ \\
\hline Benz[a]anthracene & $56-55-3$ & $1.10 \mathrm{E}-01$ & & & $8.80 \mathrm{E}-02$ & $8.80 E-02$ \\
\hline Benz[e]acephenanthrylene & $205-99-2$ & $1.10 \mathrm{E}-01$ & & & $8.80 \mathrm{E}-02$ & $8.80 E-02$ \\
\hline Indeno[1,2,3-cd]pyrene & $193-39-5$ & $1.10 \mathrm{E}-01$ & & & $8.80 \mathrm{E}-02$ & $8.80 E-02$ \\
\hline Acrylonitrile & $107-13-1$ & $2.90 \mathrm{E}-01$ & $6.80 \mathrm{E}-02$ & & $6.80 \mathrm{E}-02$ & $6.80 E-02$ \\
\hline Aniline, 2-methyl- & $95-53-4$ & $5.10 \mathrm{E}-02$ & & & & $5.10 E-02$ \\
\hline 2,3,7,8-TCDD (TEQ) & $1746-01-6$ & $3.80 \mathrm{E}-02$ & & & $3.30 \mathrm{E}-02$ & $3.30 E-02$ \\
\hline Acetamide & 60-35-5 & $2.00 \mathrm{E}-02$ & & & & $2.00 E-02$ \\
\hline Ethylenethiourea & $96-45-7$ & $1.30 \mathrm{E}-02$ & & & & $1.30 \mathrm{E}-02$ \\
\hline Formaldehyde & $50-00-0$ & $6.00 \mathrm{E}-03$ & $1.30 \mathrm{E}-02$ & & $1.30 \mathrm{E}-02$ & $1.30 \mathrm{E}-02$ \\
\hline Lead & $7439-92-1$ & $1.20 \mathrm{E}-02$ & & & & $1.20 \mathrm{E}-02$ \\
\hline Benzo[k]fluoranthene & 207-08-9 & $1.10 \mathrm{E}-01$ & & & $8.80 \mathrm{E}-03$ & $8.80 E-03$ \\
\hline Carbazole & 86-74-8 & & & $5.70 \mathrm{E}-03$ & & $5.70 \mathrm{E}-03$ \\
\hline Vinyl chloride & $75-01-4$ & $7.80 \mathrm{E}-02$ & $4.40 \mathrm{E}-03$ & & $8.80 \mathrm{E}-03$ & $4.40 E-03$ \\
\hline Propylene oxide & $75-56-9$ & $3.70 \mathrm{E}-03$ & $3.70 \mathrm{E}-03$ & & $3.70 \mathrm{E}-03$ & $3.70 E-03$ \\
\hline
\end{tabular}




\begin{tabular}{|c|c|c|c|c|c|c|}
\hline Agent & CAS no. & $\begin{array}{c}\text { Cal. EPA } \\
\text { inhal. unit risk a } \\
\left(\mathrm{mg} / \mathrm{m}^{3}\right)^{-1}\end{array}$ & $\begin{array}{c}\text { US EPA } \\
\text { inhal. unit risk } \\
\left(\mathrm{mg} / \mathrm{m}^{3}\right)^{-1}\end{array}$ & $\begin{array}{c}\begin{array}{c}\text { NATA } 1996 \\
\text { inhal. unit risk } \\
\left(\mathrm{mg} / \mathrm{m}^{3}\right)^{-1}\end{array} \\
\end{array}$ & $\begin{array}{c}\text { ORNL } \\
\begin{array}{c}\text { inhal. unit risk e } \\
\left(\mathrm{mg} / \mathrm{m}^{3}\right)^{-1}\end{array}\end{array}$ & $\begin{array}{c}\text { Selected } \\
\text { inhal. unit risk } \\
\left(\mathrm{mg} / \mathrm{m}^{3}\right)^{-1}\end{array}$ \\
\hline Di(2-ethylhexyl) phthalate & $117-81-7$ & $2.40 \mathrm{E}-03$ & & & & $2.40 E-03$ \\
\hline Acetaldehyde & $75-07-0$ & $2.70 \mathrm{E}-03$ & $2.20 \mathrm{E}-03$ & & $2.20 \mathrm{E}-03$ & $2.20 E-03$ \\
\hline Benzene & $71-43-2$ & $2.90 \mathrm{E}-02$ & $2.20 \mathrm{E}-03$ & & $7.80 \mathrm{E}-03$ & $2.20 \mathrm{E}-03$ \\
\hline Aniline & $62-53-3$ & $1.60 \mathrm{E}-03$ & & & & $1.60 E-03$ \\
\hline Chrysene & $218-01-9$ & $1.10 \mathrm{E}-02$ & & & $8.80 \mathrm{E}-04$ & $8.80 \mathrm{E}-04$ \\
\hline Polonium-210 (pCi $)^{f}$ & $7440-08-6$ & & & & $1.08 E-08^{f}$ & $1.08 E-08^{f}$ \\
\hline
\end{tabular}

\footnotetext{
${ }^{a}$ California EPA Office of Environmental Health Hazard Assessment/Risk Assessment: California cancer potency values, downloaded as a PDF file on June 6, 2002 from www.oehha.ca.gov/risk/chemicalDB.

${ }^{b}$ US EPA Integrated Risk Information System (IRIS): Inhalation RfCs and air unit risk factors, downloaded on June 6, 2002 from www.epa.gov/iris.

${ }^{\circ}$ US EPA: National-scale air toxics assessment for 1996, EPA-453/R-01-003 (2001) Appendix H, Table 1, downloaded on June 6, 2002 from www.epa.gov/ttn/atw/nata/natsa4.html.

d US EPA: Health effects assessment summary tables, EPA-540-R-97-036 (1997).

e US Department of Energy, Office of Environmental Management, Oak Ridge Operations Office: Risk assessment information system, Risk assessment tools, June 2002, nonradionuclides in Excel spreadsheet and radionuclides in Excel spreadsheet, downloaded on June 20 , 2002 from http://risk.Isd.ornl.gov/tox/tox values.shtml.

${ }^{\mathrm{f}}$ Inhalation units for ${ }^{210} \mathrm{Po}$ are given in risk/pCi.
}

\section{QUALITATIVE RANKING OF EXCESS LIFETIME CANCER RISK}

To aid the reader in interpretation of the estimated lifetime cancer risk, Table 8 prepared by the NEW YORK STATE DEPARTMENT OF HEALTH (78) is presented.

Additionally, the reference states, "An estimated increased excess lifetime cancer risk is not a specific estimate of expected cancers. Rather, it is a plausible upper bound estimate [emphasis added] of the probability that a person may develop cancer sometime in his or her lifetime following exposure to that contaminant."

For the compounds listed in Table 7 that have estimated incremental lifetime cancer risk greater than one in a million, i.e., 1.00E-06, seventeen toxicants use old yield data for nonfiltered cigarettes and two of these seventeen compounds, DDT and $N$-nitrosodiethanolamine, are obsolete MSS toxicants.

\section{SELECTION OFNON-CANCER HEALTHEFFECTS TOXICITY VALUES FOR RANKING MSS TOXICANTS}

Just as there is a variety of sources for cancer potency values, there are multiple sources of data for non-cancer effects. From VorHEES et al. (31) we get the following definition:

The toxicity criteria used to calculate potential non-cancer risk for the inhalation route of exposure are reference concentrations (RfCs). An RfC is an estimate (with uncertainty spanning perhaps an order of magnitude) of a continuous inhalation exposure to the human population (including sensitive subgroups) that is likely to be without an appreciable risk of deleterious non-cancer effects during a lifetime (US EPA 1997). The smaller the RfC, the more potent the compound. RfCs are designed to provide conservative estimates of health risk that are protective for the most sensitive members of the population.
The RfC values in $\mathrm{mg} / \mathrm{m}^{3}$ were downloaded from two websites, i.e., the US EPA IRIS site and the Oak Ridge National Laboratory (ORNL) RAIS site. Both sources are fully referenced in Table 9. An additional resource was the US EPA 1997 Health Effects Summary Tables (HEAST). For the smoke toxicants listed in Table 1 there are fewer RfCs available than carcinogenic potency values. Most often the same RfC values were contained both in the IRIS and RAIS databases. All the values are shown in the following table as well as our "selected" RfC.

\section{CALCULATION OF NON-CANCER RISK FROM EXPOSURE TO MSS TOXICANTS}

Non-cancer risk potential is calculated by dividing the average daily concentration (ADC) in $\mathrm{mg} / \mathrm{m}^{3}$ of a substance by its RfC. The quotient from this division is called the hazard index (HI).

$$
\text { Hazard Index }=\frac{\mathrm{ADC}, \mathrm{mg} / \mathrm{m}^{3}}{\mathrm{RfC}, \mathrm{mg} / \mathrm{m}^{3}}
$$

Hazard indices greater than one suggest a potential for adverse health effects while indices less than one indicate that it is unlikely for even a sensitive subpopulation to experience adverse health effects. In our calculations of hazard indices for MSS toxicants, we again assumed a pack-a-day smoker and a total daily breathing volume of $20 \mathrm{~m}^{3}$. Results of our analysis are shown in Table 10.

There were RfC values available for 24 MSS toxicants listed in Table 1. The estimated HI for eight smoke toxicants exceeded the ratio of one. Estimates for two of these compounds, hydrogen sulfide and nitrobenzene, are based upon old MSS yield data. It appears from the data in Table 10 that acrolein has the greatest potential for causing adverse non-cancer health effects. 
Table 7. Estimated chemical specific incremental lifetime cancer risk for a pack-a-day smoker. Agents in bold have been included in previous lists of cigarette MSS toxicants, but no longer appear to be relevant. See footnotes of Table 1 for details.

\begin{tabular}{|c|c|c|c|c|c|c|}
\hline Agent & CAS no. & $\begin{array}{c}\text { Comp. cig value } \\
\mu \mathrm{g} / \mathrm{cig}\end{array}$ & $\begin{array}{c}A D C^{\text {a }} 20 \mathrm{cig} \\
\mu \mathrm{g} / \mathrm{m}^{3}\end{array}$ & $\begin{array}{c}\mathrm{ADC}_{\text {lifi }}{ }^{\mathrm{b}} \\
\mu \mathrm{g} / \mathrm{m}^{3}\end{array}$ & $\begin{array}{c}\text { Inhal. unit risk } \\
\left(\mathrm{mg} / \mathrm{m}^{3}\right)^{-1}\end{array}$ & $\begin{array}{c}\text { Incremental lifetime } \\
\text { cancer risk }\end{array}$ \\
\hline 1,3-Butadiene & $106-99-0$ & $4.10 \mathrm{E}+01$ & $4.10 \mathrm{E}+01$ & $2.05 E+01$ & $2.80 \mathrm{E}-01$ & $5.74 \mathrm{E}-03$ \\
\hline Ethyl carbamate $\{$ urethane $\}$ & 51-79-6 & $3.80 \mathrm{E}+01$ & $3.80 E+01$ & $1.90 \mathrm{E}+01$ & $2.90 \mathrm{E}-01$ & $5.51 \mathrm{E}-03$ \\
\hline Propane, 2-nitro- & $79-46-9$ & $2.20 \mathrm{E}+00$ & $2.20 E+00$ & $1.10 \mathrm{E}+00$ & $2.70 \mathrm{E}+00$ & $2.97 \mathrm{E}-03$ \\
\hline Acrylamide & $79-06-1$ & $2.20 \mathrm{E}+00$ & $2.20 \mathrm{E}+00$ & $1.10 \mathrm{E}+00$ & $1.30 \mathrm{E}+00$ & $1.43 E-03$ \\
\hline Acetaldehyde & $75-07-0$ & $6.40 \mathrm{E}+02$ & $6.40 E+02$ & $3.20 \mathrm{E}+02$ & $2.20 \mathrm{E}-03$ & $7.04 \mathrm{E}-04$ \\
\hline Quinoline & $91-22-5$ & $3.15 E-01$ & $3.15 E-01$ & $1.58 \mathrm{E}-01$ & $3.40 \mathrm{E}+00$ & $5.36 \mathrm{E}-04$ \\
\hline Acrylonitrile & $107-13-1$ & $1.39 \mathrm{E}+01$ & $1.39 \mathrm{E}+01$ & $6.95 E+00$ & $6.80 \mathrm{E}-02$ & $4.73 \mathrm{E}-04$ \\
\hline Ethylene oxide & $75-21-8$ & $7.00 \mathrm{E}+00$ & $7.00 \mathrm{E}+00$ & $3.50 \mathrm{E}+00$ & $1.00 \mathrm{E}-01$ & $3.50 \mathrm{E}-04$ \\
\hline$N$-Nitrosopiperidine & $100-75-4$ & $2.31 \mathrm{E}-01$ & $2.31 \mathrm{E}-01$ & $1.16 \mathrm{E}-01$ & $2.70 \mathrm{E}+00$ & $3.12 \mathrm{E}-04$ \\
\hline Formaldehyde & 50-00-0 & $2.31 \mathrm{E}+01$ & $2.31 E+01$ & $1.16 \mathrm{E}+01$ & $1.30 \mathrm{E}-02$ & $1.50 \mathrm{E}-04$ \\
\hline$N$-Nitrosodimethylamine & $62-75-9$ & $1.91 \mathrm{E}-02$ & $1.91 \mathrm{E}-02$ & $9.55 E-03$ & $1.40 \mathrm{E}+01$ & $1.34 \mathrm{E}-04$ \\
\hline Hydrazine & $302-01-2$ & $4.30 \mathrm{E}-02$ & $4.30 \mathrm{E}-02$ & $2.15 E-02$ & $4.90 \mathrm{E}+00$ & $1.05 E-04$ \\
\hline Cadmium & $7440-43-9$ & $6.73 \mathrm{E}-02$ & $6.73 \mathrm{E}-02$ & $3.37 \mathrm{E}-02$ & $1.80 \mathrm{E}+00$ & $6.06 \mathrm{E}-05$ \\
\hline$N$-Nitrosodiethylamine & 55-18-5 & $2.80 \mathrm{E}-03$ & $2.80 \mathrm{E}-03$ & $1.40 \mathrm{E}-03$ & $4.30 \mathrm{E}+01$ & $6.02 \mathrm{E}-05$ \\
\hline DDT & $50-29-3$ & $1.20 \mathrm{E}+00$ & $1.20 \mathrm{E}+00$ & $6.00 \mathrm{E}-01$ & $9.70 \mathrm{E}-02$ & $5.82 \mathrm{E}-05$ \\
\hline Benzene & $71-43-2$ & $4.79 \mathrm{E}+01$ & $4.79 \mathrm{E}+01$ & $2.40 E+01$ & $2.20 E-03$ & $5.27 \mathrm{E}-05$ \\
\hline Polonium-210 (pCi) & $7440-08-6$ & $1.60 \mathrm{E}-02^{\mathrm{c}}$ & $3.20 \mathrm{E}-01^{\mathrm{d}}$ & $1.60 \mathrm{E}-01^{\mathrm{e}}$ & $1.08 E-08^{f}$ & $4.42 \mathrm{E}-05^{g}$ \\
\hline$N$-Nitrosoethylmethylamine & $10595-95-6$ & $1.30 \mathrm{E}-02$ & $1.30 \mathrm{E}-02$ & $6.50 \mathrm{E}-03$ & $6.30 \mathrm{E}+00$ & $4.10 \mathrm{E}-05$ \\
\hline Acetamide & $60-35-5$ & $3.97 \mathrm{E}+00$ & $3.97 \mathrm{E}+00$ & $1.99 \mathrm{E}+00$ & $2.00 \mathrm{E}-02$ & $3.97 \mathrm{E}-05$ \\
\hline$N$-Nitrosodi- $n$-butylamine & $924-16-3$ & $3.00 \mathrm{E}-02$ & $3.00 \mathrm{E}-02$ & $1.50 \mathrm{E}-02$ & $1.60 \mathrm{E}+00$ & $2.40 \mathrm{E}-05$ \\
\hline Di(2-ethylhexyl) phthalate & 117-81-7 & $2.00 \mathrm{E}+01$ & $2.00 \mathrm{E}+01$ & $1.00 \mathrm{E}+01$ & $2.40 \mathrm{E}-03$ & $2.40 E-05$ \\
\hline$N$-Nitrosonornicotine & $16543-55-8$ & $1.15 \mathrm{E}-01$ & $1.15 \mathrm{E}-01$ & $5.75 E-02$ & $4.00 \mathrm{E}-01$ & $2.30 \mathrm{E}-05$ \\
\hline $\mathrm{A} \propto \mathrm{C}$ & $26148-68-5$ & $2.60 \mathrm{E}-01$ & $2.60 \mathrm{E}-01$ & $1.30 \mathrm{E}-01$ & $1.14 \mathrm{E}-01$ & $1.48 \mathrm{E}-05$ \\
\hline Arsenic & $7440-38-2$ & $5.80 \mathrm{E}-03$ & $5.80 \mathrm{E}-03$ & $2.90 \mathrm{E}-03$ & $4.30 \mathrm{E}+00$ & $1.25 \mathrm{E}-05$ \\
\hline Biphenyl, 4-amino- & $92-67-1$ & $4.00 \mathrm{E}-03$ & $4.00 \mathrm{E}-03$ & $2.00 \mathrm{E}-03$ & $6.00 \mathrm{E}+00$ & $1.20 \mathrm{E}-05$ \\
\hline Chromium VI & $1333-82-0$ & $1.32 \mathrm{E}-03$ & $1.32 \mathrm{E}-03$ & $6.60 \mathrm{E}-04$ & $1.20 \mathrm{E}+01$ & $7.92 \mathrm{E}-06$ \\
\hline $\mathrm{MeA} \propto \mathrm{C}$ & $68006-83-7$ & $3.70 \mathrm{E}-02$ & $3.70 \mathrm{E}-02$ & $1.85 \mathrm{E}-02$ & $3.40 \mathrm{E}-01$ & $6.29 \mathrm{E}-06$ \\
\hline Aniline, 2-methyl- & 95-53-4 & $2.00 \mathrm{E}-01$ & $2.00 \mathrm{E}-01$ & $1.00 \mathrm{E}-01$ & $5.10 \mathrm{E}-02$ & $5.10 \mathrm{E}-06$ \\
\hline$N$-Nitrosopyrrolidine & $930-55-2$ & $1.40 \mathrm{E}-02$ & $1.40 \mathrm{E}-02$ & $7.00 \mathrm{E}-03$ & $6.10 \mathrm{E}-01$ & $4.27 \mathrm{E}-06$ \\
\hline Chrysene, 5-methyl- & $3697-24-3$ & $7.60 \mathrm{E}-03$ & $7.60 \mathrm{E}-03$ & $3.80 \mathrm{E}-03$ & $1.10 \mathrm{E}+00$ & $4.18 \mathrm{E}-06$ \\
\hline Naphthalene, 2-amino- & 91-59-8 & $1.11 \mathrm{E}-02$ & $1.11 \mathrm{E}-02$ & $5.55 E-03$ & $5.14 \mathrm{E}-01$ & $2.85 \mathrm{E}-06$ \\
\hline Carbazole & $86-74-8$ & $1.00 \mathrm{E}+00$ & $1.00 \mathrm{E}+00$ & $5.00 \mathrm{E}-01$ & $5.70 \mathrm{E}-03$ & $2.85 E-06$ \\
\hline Benzo[a]pyrene & $50-32-8$ & $5.70 \mathrm{E}-03$ & $5.70 \mathrm{E}-03$ & $2.85 E-03$ & $8.80 \mathrm{E}-01$ & $2.51 \mathrm{E}-06$ \\
\hline Trp-P-1 & $62450-06-0$ & $5.00 \mathrm{E}-04$ & $5.00 E-04$ & $2.50 \mathrm{E}-04$ & $7.40 \mathrm{E}+00$ & $1.85 \mathrm{E}-06$ \\
\hline N-Nitrosodiethanolamine & $1116-54-7$ & $4.30 \mathrm{E}-03$ & $4.30 \mathrm{E}-03$ & $2.15 E-03$ & $8.00 \mathrm{E}-01$ & $1.72 \mathrm{E}-06$ \\
\hline Benzo[]fluoranthene & $205-82-3$ & $2.10 \mathrm{E}-02$ & $2.10 \mathrm{E}-02$ & $1.05 E-02$ & $1.10 \mathrm{E}-01$ & $1.16 \mathrm{E}-06$ \\
\hline$N$-Nitrosodi- $n$-propylamine & $621-64-7$ & $1.00 \mathrm{E}-03$ & $1.00 \mathrm{E}-03$ & $5.00 \mathrm{E}-04$ & $2.00 \mathrm{E}+00$ & $1.00 \mathrm{E}-06$ \\
\hline Nickel & $7440-02-0$ & $5.58 \mathrm{E}-03$ & $5.58 \mathrm{E}-03$ & $2.79 \mathrm{E}-03$ & $2.40 \mathrm{E}-01$ & $6.70 \mathrm{E}-07$ \\
\hline Glu-P-1 & $67730-11-4$ & $8.90 \mathrm{E}-04$ & $8.90 \mathrm{E}-04$ & $4.45 \mathrm{E}-04$ & $1.40 \mathrm{E}+00$ & $6.23 \mathrm{E}-07$ \\
\hline Beryllium & $7440-41-7$ & $5.00 \mathrm{E}-04$ & $5.00 \mathrm{E}-04$ & $2.50 E-04$ & $2.40 \mathrm{E}+00$ & $6.00 \mathrm{E}-07$ \\
\hline Benz[a]anthracene & $56-55-3$ & $1.24 \mathrm{E}-02$ & $1.24 \mathrm{E}-02$ & $6.20 \mathrm{E}-03$ & $8.80 \mathrm{E}-02$ & $5.46 \mathrm{E}-07$ \\
\hline Aniline & 62-53-3 & $6.55 E-01$ & $6.55 E-01$ & $3.28 E-01$ & $1.60 \mathrm{E}-03$ & $5.24 \mathrm{E}-07$ \\
\hline Trp-P-2 & $62450-07-1$ & $1.10 \mathrm{E}-03$ & $1.10 \mathrm{E}-03$ & $5.50 \mathrm{E}-04$ & $9.10 \mathrm{E}-01$ & $5.01 \mathrm{E}-07$ \\
\hline $7 H$-Dibenzo $[c, g]$ carbazole & 194-59-2 & $7.00 \mathrm{E}-04$ & $7.00 \mathrm{E}-04$ & $3.50 \mathrm{E}-04$ & $1.10 \mathrm{E}+00$ & $3.85 E-07$ \\
\hline Benz[e]acephenanthrylene & 205-99-2 & $5.50 \mathrm{E}-03$ & $5.50 \mathrm{E}-03$ & $2.75 E-03$ & $8.80 \mathrm{E}-02$ & $2.42 \mathrm{E}-07$ \\
\hline Lead & $7439-92-1$ & $3.91 \mathrm{E}-02$ & $3.91 \mathrm{E}-02$ & $1.96 \mathrm{E}-02$ & $1.20 \mathrm{E}-02$ & $2.35 \mathrm{E}-07$ \\
\hline Propylene oxide & $75-56-9$ & $1.00 \mathrm{E}-01$ & $1.00 \mathrm{E}-01$ & $5.00 \mathrm{E}-02$ & $3.70 \mathrm{E}-03$ & $1.85 \mathrm{E}-07$ \\
\hline Dibenz[a,h]anthracene & $53-70-3$ & $4.00 \mathrm{E}-04$ & $4.00 E-04$ & $2.00 \mathrm{E}-04$ & $8.80 \mathrm{E}-01$ & $1.76 \mathrm{E}-07$ \\
\hline Glu-P-2 & $67730-10-3$ & $8.80 \mathrm{E}-04$ & $8.80 \mathrm{E}-04$ & $4.40 \mathrm{E}-04$ & $4.00 \mathrm{E}-01$ & $1.76 \mathrm{E}-07$ \\
\hline Ethylenethiourea & 96-45-7 & $2.70 \mathrm{E}-02$ & $2.70 \mathrm{E}-02$ & $1.35 E-02$ & $1.30 \mathrm{E}-02$ & $1.76 \mathrm{E}-07$ \\
\hline Indeno[1,2,3-cd]pyrene & $193-39-5$ & $3.50 \mathrm{E}-03$ & $3.50 \mathrm{E}-03$ & $1.75 E-03$ & $8.80 \mathrm{E}-02$ & $1.54 \mathrm{E}-07$ \\
\hline Dibenz $[a, j]$ acridine & $224-42-0$ & $2.72 \mathrm{E}-03$ & $2.72 \mathrm{E}-03$ & $1.36 \mathrm{E}-03$ & $1.10 \mathrm{E}-01$ & $1.50 \mathrm{E}-07$ \\
\hline Vinyl chloride & 75-01-4 & $3.00 \mathrm{E}-02$ & $3.00 \mathrm{E}-02$ & $1.50 \mathrm{E}-02$ & $4.40 \mathrm{E}-03$ & $6.60 \mathrm{E}-08$ \\
\hline $\mathrm{IQ}$ & $76180-96-6$ & $3.00 \mathrm{E}-04$ & $3.00 \mathrm{E}-04$ & $1.50 \mathrm{E}-04$ & $4.00 \mathrm{E}-01$ & $6.00 \mathrm{E}-08$ \\
\hline Chrysene & 218-01-9 & $1.36 \mathrm{E}-02$ & $1.36 \mathrm{E}-02$ & $6.80 \mathrm{E}-03$ & $8.80 \mathrm{E}-04$ & $5.98 \mathrm{E}-09$ \\
\hline
\end{tabular}




\begin{tabular}{l|r|c|c|c|c|c}
\hline Agent & CAS no. & $\begin{array}{c}\text { Comp. cig value } \\
\mu \mathrm{g} / \mathrm{cig}\end{array}$ & $\begin{array}{c}\mathrm{ADC} \mathrm{a}^{20} \mathrm{cig} \\
\mu \mathrm{g} / \mathrm{m}^{3}\end{array}$ & $\begin{array}{c}\mathrm{ADC}_{\text {life }}{ }^{\mathrm{b}} \\
\mu \mathrm{g} / \mathrm{m}^{3}\end{array}$ & $\begin{array}{c}\text { Inhal. unit risk } \\
\left(\mathrm{mg} / \mathrm{m}^{3}\right)^{-1}\end{array}$ & $\begin{array}{c}\text { Incremental lifetime } \\
\text { cancer risk }\end{array}$ \\
\hline Benzo[ $k$ ]fluoranthene & $207-08-9$ & $1.30 \mathrm{E}-03$ & $1.30 \mathrm{E}-03$ & $6.50 \mathrm{E}-04$ & $8.80 \mathrm{E}-03$ & $5.72 \mathrm{E}-09$ \\
Dibenz[a, $\boldsymbol{h}$ ]acridine & $226-36-8$ & $1.00 \mathrm{E}-04$ & $1.00 \mathrm{E}-04$ & $5.00 \mathrm{E}-05$ & $1.10 \mathrm{E}-01$ & $5.50 \mathrm{E}-09$ \\
$2,3,7,8-T C D D(T E Q)$ & $1746-01-6$ & $1.26 \mathrm{E}-06$ & $1.26 \mathrm{E}-06$ & $6.30 \mathrm{E}-07$ & $3.30 \mathrm{E}-02$ & $2.08 \mathrm{E}-11$ \\
\hline
\end{tabular}

${ }^{a}$ Calculation of average daily concentration (ADC) assumes smoking $20 \mathrm{cig} / \mathrm{day}$ and a breathing volume of $20 \mathrm{~m}^{3}$. ADC $=$ comparison cig value, $\mu \mathrm{g} / \mathrm{cig} \times 20 \mathrm{cig} /$ day $\div$ breathing volume, $20 \mathrm{~m}^{3}$.

${ }^{b}$ The lifetime average daily exposure $\left(A D C_{\text {life }}\right)$ is calculated as follows: $A D C_{\text {life }}, \mu \mathrm{g} / \mathrm{m}^{3}=A D C \times 35$ years smoking $\div 70$ year average lifetime

${ }^{c}$ The comparison cigarette value for ${ }^{210} \mathrm{Po}$ has units of picocuries per cigarette.

${ }^{\mathrm{d}}$ The ADC for ${ }^{210} \mathrm{Po}$ has units of picocuries per day.

${ }^{\mathrm{e}}$ The $\mathrm{ADC}_{\text {life }}$ for ${ }^{210} \mathrm{Po}$ is calculated as follows: $\mathrm{ADC}_{\text {life }}=3.20 \mathrm{E}-01 \mathrm{pCi} / \mathrm{day} \times 365 \mathrm{day} / \mathrm{yr} \times 35$ smoking years and has units of $\mathrm{pCi}$.

${ }^{\mathrm{f}}$ The Unit Risk (morbidity) for ${ }^{210} \mathrm{Po}$ has units of risk/pCi.

$\mathrm{g}$ The estimated incremental lifetime cancer risk ILCR ${ }_{\mathrm{Po-210}}$ from ${ }^{210} \mathrm{Po}$ is calculated as follows: ILCR $\mathrm{Po}-210=1.60 \mathrm{E}-02 \mathrm{pCi} / \mathrm{cig} \times 20$ $\mathrm{cig} / \mathrm{day} \times 365 \mathrm{day} / \mathrm{yr} \times 35$ smoking years $\times 1.08 \mathrm{E}-08 \mathrm{risk} / \mathrm{pCi}$.

Table 8. Qualitative ranking of excess lifetime cancer risk

\begin{tabular}{ll}
\hline Risk ratio & Qualitative descriptor \\
\hline $\begin{array}{l}\text { Equal to or less than one in a million } \\
\text { Greater than one in a million to less }\end{array}$ low low \\
than one in ten thousand & \\
$\begin{array}{l}\text { One in ten thousand to less than one } \\
\text { in a thousand }\end{array}$ & moderate \\
One in a thousand to less than one in & high \\
ten & \\
Equal to or greater than one in ten & very high \\
\hline
\end{tabular}

\section{THE ASSERTION OF THE GENERATION OF TOXICANTS FROM ADDITIVES}

In a previous section of our paper, we touched briefly on the assertions that tobacco additives are a source of toxicants and should be investigated accordingly. Having achieved greater "tar" reduction than the cigarette-smoking critics had originally proposed, e.g., see WYNDER (36), the Tobacco Industry unwittingly provided an alternate subject for criticism. The late 1970s, early 1980s heralded the advent of low-"tar" and ultralow-"tar" cigarettes and their acquisition of a significant share of the US cigarette market. Bases of the criticism were a) some commercial low-"tar" brands might have levels of additives much higher than the levels in previous high- and medium-"tar" cigarettes and b) the fates of many of the individual added components during the cigarette smoking process were unknown.

In the 1950s, concern was expressed about the pyrogenesis of PAHs from tobacco components (36) and their possible pyrogenesis from additives. However, the proponents of possible problems with tobacco additives became much more vocal about them when the nearly $70 \%$ reduction in sales-weighted MSS “tar" delivery between 1955 and 1985 not only answered the criticisms voiced in the late 1950s, early 1960 s but met the goal set by others, i.e., the halving of "tar" delivery as a means to lower lung cancer incidence in cigarette smokers (36).
In 1980, LAVOIE et al. (79) wrote:

The development of the low-tar, low-nicotine cigarette required cigarette fillers with a potential for smoke flavor contribution to make these cigarettes acceptable to the consumer. Such products can be realized either by selecting tobaccos rich in flavor or by addition of tobacco extracts or certain plant extracts, addition of synthetic flavor compounds, or a combination of several of these factors ...

New cigarettes should be assayed for toxicity and tumorigenicity, so that the reduction of toxic and tumorigenic effects in the smoke of low-tar, low-nicotine cigarettes is not offset by the introduction of unknown factors.

Despite their criticism of the possible increased use of flavorants in the filler of low-"tar", low-nicotine cigarettes, a key part of this discussion is the admission by LAVOIE $e t$ al. that prior to 1980 , the US cigarette manufacturers had apparently achieved a "reduction of toxic and tumorigenic effects in the smoke of low-"tar," low nicotine cigarettes". In the 1979 report of the SURGEON GENERAL [see pp. 63-64 in (43)] the following was written:

[T]he trend toward low-tar, low-nicotine cigarettes and toward a reduction of undesirable volatile smoke compounds has brought about major changes in the smoke flavor of cigarettes. The use of rolled stems and reconstituted tobacco sheet admixed with leaf lamina and the use of effective filter tips are major factors inducing changes in smoke flavor. All of these developments have led to increased use of flavor additives, especially for low-tar, low-nicotine cigarettes. In fact, these new cigarettes require flavor corrections by additives in order to be acceptable to the consumer. Tobacco extracts as well as nontobacco flavors, such as licorice, cocoa, fruit, spices, and floral compositions, are used ... At present, the selection of tobacco flavor additives from the GRAS (Generally Regarded As Safe) List or from natural extracts and the screening of their smoke decomposition products for toxicity or other biological activity are not required by law and are done voluntarily by manufacturers.

Critics then asserted that the Industry's use of higher levels of flavorants in low-"tar" cigarettes might increase the hazard to the smoker because the fate of the added ingredients was not known [see RoDGMAN (16)]. No evidence was ever presented that the added flavorants actually increased the risk. 
Table 9. Non-cancer reference concentrations for some toxicants in cigarette mainstream smoke

\begin{tabular}{|c|c|c|c|c|c|}
\hline Agent & CAS no. & $\begin{array}{l}\text { EPA RfC a } \\
\mathrm{mg} / \mathrm{m}^{3}\end{array}$ & $\begin{array}{c}\text { HEAST RfC } \\
\mathrm{mg} / \mathrm{m}^{3}\end{array}$ & $\begin{array}{c}\text { ORNL RfC } \\
\mathrm{mg} / \mathrm{m}^{3}\end{array}$ & $\begin{array}{c}\text { Selected RfC } \\
\mathrm{mg} / \mathrm{m}^{3}\end{array}$ \\
\hline 2-Butanone & $78-93-3$ & & $1.00 \mathrm{E}+00$ & $1.00 \mathrm{E}+00$ & $1.00 \mathrm{E}+00$ \\
\hline Methanol & $67-56-1$ & $1.00 \mathrm{E}+00$ & & & $1.00 \mathrm{E}+00$ \\
\hline Styrene $\{$ benzene, ethenyl- $\}$ & $100-42-4$ & $1.00 \mathrm{E}+00$ & & & $1.00 \mathrm{E}+00$ \\
\hline Carbon disulfide & $75-15-0$ & $7.00 \mathrm{E}-01$ & & $7.00 \mathrm{E}-01$ & $7.00 \mathrm{E}-01$ \\
\hline Toluene & $108-88-3$ & $4.00 \mathrm{E}-01$ & & $4.00 \mathrm{E}-01$ & $4.00 \mathrm{E}-01$ \\
\hline Vinyl acetate & $108-05-4$ & $2.00 \mathrm{E}-01$ & & $2.00 \mathrm{E}-01$ & $2.00 \mathrm{E}-01$ \\
\hline Ammonia & $7664-41-7$ & $1.00 \mathrm{E}-01$ & & $1.00 \mathrm{E}-01$ & $1.00 \mathrm{E}-01$ \\
\hline Vinyl chloride & 75-01-4 & $1.00 \mathrm{E}-01$ & & $1.00 \mathrm{E}-01$ & $1.00 \mathrm{E}-01$ \\
\hline Acetonitrile & $75-05-8$ & $6.00 \mathrm{E}-02$ & & $6.00 \mathrm{E}-02$ & $6.00 \mathrm{E}-02$ \\
\hline Furfural & $98-01-1$ & & & $5.00 \mathrm{E}-02$ & $5.00 \mathrm{E}-02$ \\
\hline Propylene oxide & $75-56-9$ & $3.00 \mathrm{E}-02$ & & $3.00 \mathrm{E}-02$ & $3.00 \mathrm{E}-02$ \\
\hline Propane, 2-nitro- & $79-46-9$ & $2.00 \mathrm{E}-02$ & & $2.00 \mathrm{E}-02$ & $2.00 \mathrm{E}-02$ \\
\hline Acetaldehyde & $75-07-0$ & $9.00 \mathrm{E}-03$ & & $9.00 E-03$ & $9.00 E-03$ \\
\hline Hydrogen cyanide & $74-90-8$ & $3.00 \mathrm{E}-03$ & & $3.00 \mathrm{E}-03$ & $3.00 \mathrm{E}-03$ \\
\hline Naphthalene & $91-20-3$ & $3.00 \mathrm{E}-03$ & & $3.00 \mathrm{E}-03$ & $3.00 \mathrm{E}-03$ \\
\hline Acrylonitrile & $107-13-1$ & $2.00 \mathrm{E}-03$ & & $2.00 \mathrm{E}-03$ & $2.00 \mathrm{E}-03$ \\
\hline Nitrobenzene & $98-95-3$ & & & $2.00 \mathrm{E}-03$ & $2.00 \mathrm{E}-03$ \\
\hline Aniline & 62-53-3 & $1.00 \mathrm{E}-03$ & & $1.00 \mathrm{E}-03$ & $1.00 \mathrm{E}-03$ \\
\hline Hydrogen sulfide & $7783-06-4$ & $1.00 \mathrm{E}-03$ & & $1.00 \mathrm{E}-03$ & $1.00 \mathrm{E}-03$ \\
\hline Hydroquinone & $123-31-9$ & & $1.00 \mathrm{E}-03$ & & $1.00 \mathrm{E}-03$ \\
\hline Mercury & $7439-97-6$ & $3.00 \mathrm{E}-04$ & & $3.00 \mathrm{E}-04$ & $3.00 \mathrm{E}-04$ \\
\hline Acrolein & $107-02-8$ & $2.00 \mathrm{E}-05$ & & $2.00 \mathrm{E}-05$ & $2.00 \mathrm{E}-05$ \\
\hline Beryllium & $7440-41-7$ & $2.00 \mathrm{E}-05$ & & & $2.00 \mathrm{E}-05$ \\
\hline Chromium VI & $1333-82-0$ & $8.00 \mathrm{E}-06$ & & $1.00 \mathrm{E}-04$ & $8.00 \mathrm{E}-06$ \\
\hline
\end{tabular}

a US EPA Integrated Risk Information System (IRIS): Inhalation RfCs and air unit risk factors, downloaded on June 6, 2002 from www.epa.gov/iris.

US EPA: Health effects assessment summary tables, EPA-540-R-97-036.

c US Department of Energy, Office of Environmental Management, Oak Ridge Operations Office: Risk assessment information system, Risk assessment tools, June 2002 nonradionuclides in Excel spreadsheet, downloaded on June 20, 2002 from http://risk.Isd.ornl.gov/tox/tox_values.shtml.

Table 10. Estimated chemical specific non-cancer risks for a pack-a-day smoker

\begin{tabular}{|c|c|c|c|c|c|}
\hline Agent & CAS no. & $\begin{array}{c}\text { Comp. cig value } \\
\mu \mathrm{g} / \mathrm{cig}\end{array}$ & $\begin{array}{c}\mathrm{ADC}^{\mathrm{a}} 20 \mathrm{cig} \\
\mu \mathrm{g} / \mathrm{m}^{3}\end{array}$ & $\begin{array}{c}\text { Inhal. RfC } \\
\mathrm{mg} / \mathrm{m}^{3}\end{array}$ & $\begin{array}{l}\text { Hazard } \\
\text { index }{ }^{\text {b }}\end{array}$ \\
\hline Acrolein & $107-02-8$ & $6.50 \mathrm{E}+01$ & $6.50 \mathrm{E}+01$ & $2.00 \mathrm{E}-05$ & 3250.0000 \\
\hline Hydrogen sulfide & $7783-06-4$ & $9.00 \mathrm{E}+01$ & $9.00 \mathrm{E}+01$ & $1.00 \mathrm{E}-03$ & 90.0000 \\
\hline Acetaldehyde & 75-07-0 & $6.40 \mathrm{E}+02$ & $6.40 \mathrm{E}+02$ & $9.00 \mathrm{E}-03$ & 71.1111 \\
\hline Hydrogen cyanide & $74-90-8$ & $1.65 E+02$ & $1.65 E+02$ & $3.00 \mathrm{E}-03$ & 55.0000 \\
\hline Hydroquinone & $123-31-9$ & $4.29 \mathrm{E}+01$ & $4.29 \mathrm{E}+01$ & $1.00 \mathrm{E}-03$ & 42.9000 \\
\hline Nitrobenzene & $98-95-3$ & $2.50 \mathrm{E}+01$ & $2.50 E+01$ & $2.00 \mathrm{E}-03$ & 12.5000 \\
\hline Acrylonitrile & $107-13-1$ & $1.39 \mathrm{E}+01$ & $1.39 \mathrm{E}+01$ & $2.00 \mathrm{E}-03$ & 6.9500 \\
\hline Acetonitrile & $75-05-8$ & $1.00 \mathrm{E}+02$ & $1.00 \mathrm{E}+02$ & $6.00 \mathrm{E}-02$ & 1.6667 \\
\hline Aniline & $62-53-3$ & $6.55 \mathrm{E}-01$ & $6.55 \mathrm{E}-01$ & $1.00 \mathrm{E}-03$ & 0.6550 \\
\hline Toluene & $108-88-3$ & $9.04 \mathrm{E}+01$ & $9.04 \mathrm{E}+01$ & $4.00 \mathrm{E}-01$ & 0.2260 \\
\hline Methanol & 67-56-1 & $1.80 \mathrm{E}+02$ & $1.80 E+02$ & $1.00 \mathrm{E}+00$ & 0.1800 \\
\hline Chromium VI & $1333-82-0$ & $1.32 \mathrm{E}-03$ & $1.32 \mathrm{E}-03$ & $8.00 \mathrm{E}-06$ & 0.1650 \\
\hline Ammonia & $7664-41-7$ & $1.60 \mathrm{E}+01$ & $1.60 \mathrm{E}+01$ & $1.00 \mathrm{E}-01$ & 0.1600 \\
\hline Naphthalene & $91-20-3$ & $3.42 \mathrm{E}-01$ & $3.42 \mathrm{E}-01$ & $3.00 \mathrm{E}-03$ & 0.1140 \\
\hline Propane, 2-nitro- & $79-46-9$ & $2.20 \mathrm{E}+00$ & $2.20 \mathrm{E}+00$ & $2.00 \mathrm{E}-02$ & 0.1100 \\
\hline 2-Butanone & $78-93-3$ & $9.00 \mathrm{E}+01$ & $9.00 E+01$ & $1.00 \mathrm{E}+00$ & 0.0900 \\
\hline Furfural & 98-01-1 & $1.40 \mathrm{E}+00$ & $1.40 \mathrm{E}+00$ & $5.00 \mathrm{E}-02$ & 0.0280 \\
\hline Beryllium & $7440-41-7$ & $5.00 \mathrm{E}-04$ & $5.00 \mathrm{E}-04$ & $2.00 \mathrm{E}-05$ & 0.0250 \\
\hline Vinyl acetate & $108-05-4$ & $4.00 \mathrm{E}+00$ & $4.00 \mathrm{E}+00$ & $2.00 \mathrm{E}-01$ & 0.0200 \\
\hline Mercury & $7439-97-6$ & $5.96 \mathrm{E}-03$ & $5.96 \mathrm{E}-03$ & $3.00 \mathrm{E}-04$ & 0.0199 \\
\hline Styrene $\{$ benzene, ethenyl-\} & $100-42-4$ & $7.60 \mathrm{E}+00$ & $7.60 \mathrm{E}+00$ & $1.00 \mathrm{E}+00$ & 0.0076 \\
\hline Propylene oxide & $75-56-9$ & $1.00 \mathrm{E}-01$ & $1.00 \mathrm{E}-01$ & $3.00 \mathrm{E}-02$ & 0.0033 \\
\hline Carbon disulfide & $75-15-0$ & $2.00 \mathrm{E}+00$ & $2.00 E+00$ & $7.00 \mathrm{E}-01$ & 0.0029 \\
\hline Vinyl chloride & $75-01-4$ & $3.00 \mathrm{E}-02$ & $3.00 \mathrm{E}-02$ & $1.00 \mathrm{E}-01$ & 0.0003 \\
\hline
\end{tabular}

${ }^{a}$ Calculation of average daily concentration $(A D C)$ assumes smoking $20 \mathrm{cig} /$ day and a breathing volume of $20 \mathrm{~m}^{3}$. ADC = comparison cig value, $\mu \mathrm{g} / \mathrm{cig} \times 20 \mathrm{cig} /$ day $\div$ breathing volume, $20 \mathrm{~m}^{3}$.

${ }^{b}$ The potential for non-cancer health effects is evaluated by comparing the ADC to the RfC. This ratio of exposure concentration to toxicity reference concentration is termed a hazard index $(\mathrm{HI})$. $\mathrm{HI}=\mathrm{ADC}, \mu \mathrm{g} / \mathrm{m}^{3} \times \mathrm{mg} / 1,000 \mu \mathrm{g} \div \mathrm{RfC}, \mathrm{mg} / \mathrm{m}^{3}$. 
Examination of extensive laboratory data collected during the past four decades, particularly considerable unpublished data generated between the mid-1950s and the late 1970s, indicates that none of the materials used as flavorants on smoking tobacco products, particularly cigarettes marketed by a US manufacturer, imparts any significant adverse chemical or biological properties to the MSS from flavoranttreated tobacco, a conclusion reached by DoULL et al. (80) in their recent assessment of available information on nearly 600 ingredients variously used as cigarette tobacco additives in the US Tobacco Industry. Of these ingredients 460 are individual compounds, many of which have been identified in tobacco and/or smoke. Much evidence has been collected to show that the added ingredients do not adversely affect the MSS properties. The evidence includes chemical data, e.g., smoke composition and pyrolysates, biological data on inhalation, skin painting, and genotoxicity.

In more recent detailed assessments of reported chemical and biological properties for the MSSs from cigarettes fabricated with tobacco with or without one or more additives, PASCHKE et al. (81) and RODGMAN (16) reached a similar conclusion: No significant increase in the biological activity of tobacco was reported from cigarettes containing added ingredients.

Information that flavorful components in tobacco did not enhance the PAH level in MSS was provided by the study of the organic solvent extraction of tobacco. Ultimately incorporated into the process was an aqueous alcohol-hexane partition step to separate polar, more flavorful tobacco components from the lipophilic components eventually shown to be PAH precursors. When an appropriate portion of the aqueous ethanol-soluble fraction (AEF) was returned to the extracted tobacco, no difference was found in the PAH levels in the MSSs from the extracted tobacco and the AEF-treated tobacco (RoDGMAN, 16,34,82). In the mid-1950s, the identities of most polar components were unknown though it was suspected they contributed significantly to MSS flavor and aroma. No adequate fractionation system to separate highly polar compounds in a complex mixture was available, but that situation was resolved in the 1970s. With the capability to isolate and identify highly polar and volatile components of tobacco and its MSS, it was obvious that many were identical with or similar to ingredients of flavor formulations added to specific tobacco blends to impart unique smoking characteristics (Doull et al., 80).

Although chemical data for the pyrogenesis of allegedly harmful smoke components from flavorants added to the blend at microgram levels are generally not available because of the limitations of analytical methodology, indirect confirmation of the effect of such additives on at least one MSS property is available; namely, the effect of addition of a total flavor formulation to the tobacco blend on the mutagenicity, as measured in the Ames Salmonella typhimurium test system, of the MSS particulate matter collected on a Cambridge filter pad. It has also been shown that added flavorful ingredients do not have any significant adverse effect on the composition of MSS (21).

For many years, considerable thought was given to development of an accurate analytical method to determine the contribution of trace levels (a few $\mu \mathrm{g} / \mathrm{g}$ of tobacco blend) of a flavorant added to cigarette tobacco to the levels of toxicants in MSS. Limitations in analytical methodology precluded the design of an experiment whose results would be meaningful. Even studies with radiolabeled compounds had their limitations in the study of the pyrogenesis of MSS components (cf. SCHMELTZ et al., 83).

With the advent of the Ames test in the early 1970s, an alternate to the almost insurmountable task of studying individually the effect of hundreds of flavorants added to cigarette products was devised in an attempt to show the effect on smoke condensate specific mutagenicity of additives used in commercial brands. Such flavor formulations are qualitatively and quantitatively unique for each RJRT commercial brand and comprise many different individual ingredients. This is probably true for commercial brands from other manufacturers. The weight of flavorants added to the RJRT brands ranged from 1.0 to $1.5 \mathrm{mg} / \mathrm{g}$ of tobacco blend. Four sets of cigarettes for each of five commercial brands were fabricated. The levels of flavorants, casing materials, and humectants were varied as shown later in this section.

The MSS TPM from each of these 20 cigarette variations was examined for mutagenicity in the Ames test (TA1538 and TA98 strains of Salmonella typhimurium) by a contract laboratory.

\begin{tabular}{lcc}
$\begin{array}{l}\text { Cigarette } \\
\text { variation }{ }^{\text {a }}\end{array}$ & $\begin{array}{c}\text { Flavorant } \\
\text { formulation level }\end{array}$ & $\begin{array}{c}\text { Casing materials } \\
\text { and humectants }{ }^{\mathrm{c}} \text { level }\end{array}$ \\
\hline A & $\begin{array}{c}\text { usual level used } \\
\text { on brand } \\
\text { ten times the usual } \\
\text { level used on brand }\end{array}$ & $\begin{array}{c}\text { usual level used } \\
\text { on brand }\end{array}$ \\
B & 0 & 0 \\
C & 0 & $\begin{array}{c}\text { usual level used } \\
\text { on brand }\end{array}$ \\
D & 0
\end{tabular}

Because the response of the Salmonella typhimurium was linear from 0 to $500 \mu \mathrm{g} / \mathrm{plate}$ of added wet total particulate matter (WTPM), mutagenicity in revertant/plate was tabulated for the WTPM dose level of $\mu \mathrm{g} / \mathrm{plate}$. This permitted comparison of the four cigarette variations for each Salmonella typhimurium strain and for each of five commercial brands. It was concluded (84):

Although the mutagenic activities appeared to be similar, there were statistically significant differences in mutagenic activities among the sample. It appeared that generally samples A were slightly less and samples D were slightly more mutagenic than the other samples.

The results of this study are presented in detail in RODGMAN $(16,82)$.

Substantial amounts of humectants (glycerol, propylene glycol, and/or triethylene glycol) added to tobacco blends are transferred to MSS TPM and sidestream smoke (SSS) TPM (85). Analysis of humectants in MSS indicated that the FTC "tar" from commercial cigarettes contains signifi- 
cant amounts of humectants (86). Thus, it is not surprising that their removal from the additive system produces TPM with increased mutagenicity (84). The nonmutagenic humectants act as diluents for the MSS TPM toxicants produced pyrogenetically or transferred directly from tobacco to smoke during smoking. Recently, GAWORSKI et al. reported that inhalation of MSS from cigarettes with glycerol and propylene glycol, added either individually or in combination, had no significant adverse biological effects on rats (87).

It is apparent that the flavorants used in the commercial brands studied do not increase its MSS specific mutagenicity. In fact, flavorant removal increases slightly the observed mutagenicity of the WTPM. The findings from this study indicate that the additives in the flavorant formulations for five commercial products do not contribute toxicants to the smoke whose levels and potency are such that they produce abnormal increases in the specific mutagenicity as measured in the Ames test system.

To the knowledge gained in the 1950s on the effect of added compounds on the chemical composition of MSS, particularly its PAH content $(16,82)$ and in the 1970s on the effect of product flavor formulations on MSS specific mutagenicity (84) was recently added even more definitive knowledge on the effect of addition of a mixture of selected ingredients to cigarette tobacco on laboratory animals a) exposed to the resulting MSS by inhalation and $b$ ) treated via skin painting with the resulting CSC.

Among flavorants, menthol is special because its usage level is several magnitudes greater than that of any other component in the flavor formulation. Chemically, its fate during smoking was defined by NEWELL et al. (88) and JENKINS et al. (89) from studies with ${ }^{14} \mathrm{C}$-menthol. Less than $2 \%$ of the added menthol undergoes pyrolysis during smoking. Biologically, added menthol produces little change in the effects: a) In 1965, BocK et al. (90) reported no difference between the specific tumorigenicities of CSCs from non-mentholated vs. mentholated cigarettes. b) A 10fold increase in the levels of the flavorant formulation and menthol on a commercial cigarette blend produced no significant change in specific mutagenicity (84). c) In a 13-week inhalation study with rats, GAWORSKI et al. (17) reported that addition of $5000 \mathrm{ppm}$ of menthol to the blend had no substantial effect on the character or extent of the biological responses normally associated with inhalation of cigarette MSS.

Almost $77 \%$ of the items listed by Doull et al. (80) as ingredients added by the six major US cigarette manufacturers during cigarette production are individual compounds, the remaining items are mixtures, e.g., natural oils, plant extracts, oleoresins. As noted previously by DouLL $e t$ al. (80); GAWORSKI et al. (18); PASCHKE et al. (81); RUSTEMEIER et al. (21); and RODGMAN (16), the compounds may fall into one of the following categories: a) It is a component of one or more of the tobacco types [flue-cured (LlOYD et al., 91); burley (ROBERTS and ROHDE, 92); Oriental (SCHUMACHER and VESTAL, 93); Maryland (SCHUMACHER, 94)] commonly used in cigarette blends. b) It is a component of cigarette MSS (80). c) It is a component of both tobacco and tobacco smoke. d) It is a homolog or isomer of an identified tobacco and/or tobacco smoke component.
In their study of added ingredients on the biological effect of inhaled cigarette MSS, GAWORSKI et al. (18) administered to rats via inhalation the MSS from cigarettes to which 172 ingredients (129 individual compounds, 43 mixtures) had been added. Most of the ingredients are included in the Doull et al. list. From the results of their inhalation experiment, GAWORSKI et al. concluded:

[T] he addition of these flavoring ingredients to cigarette tobacco had no discernible effect on the character or extent of the biological responses normally associated with inhalation of mainstream cigarette smoke in rats.

In a similar biological study, GAWORSKI et al. (19) investigated the effect on the specific tumorigenicity of the CSC from cigarettes to which 150 ingredients (109 individual compounds, 41 mixtures) had been added. Here again, most ingredients are included in the DouLL et al. list. From the results of their skin-painting study, GAWORSKI et al. concluded:

While tumor incidence, latency and multiplicity data occasionally differed between test and comparative reference CSC groups, all effects appeared to be within normal variation for the model system. Furthermore, none of the changes appeared to be substantial enough to conclude that the tumor promotion capacity of CSC obtained from cigarettes containing tobacco with ingredients was discernibly different from the CSC obtained from reference cigarettes containing tobacco processed without ingredients.

In early 2002, four reports by CARMINES and colleagues (20-23) were published on their excellent study of the effects of ingredients added to a cigarette on the chemical and biological properties of its MSS. A total of 333 ingredients commonly used in cigarette manufacture was added to a test cigarette, representative of a commercial blended cigarette. Ingredients were added at approximately the levels normally used in commercial cigarettes and at levels several times those normally used. The MSS data vs. those from a control cigarette with no added ingredients indicated an increase in the TPM. Normalizing the yields of individual MSS ingredients to the TPM yields indicated a reduction in the majority of them. An increase in the amount relative to TPM was observed for only a few MSS components (RUSTEMEIER et al., 21). These chemical results on the MSSs are consistent with the results obtained not only in in vitro mutagenicity and cytotoxicity studies with the TPMs from the ingredienttreated and control cigarettes (ROEMER et al., 22) but also in in vivo studies with rats exposed via inhalation to the MSSs from the treated and control cigarettes (VANSCHEEUWIJCK $e t$ al., 23): The addition of the ingredients did not increase the in vitro mutagenicity or cytotoxicity of the TPMs from the ingredient-treated cigarettes or the inhalation toxicity to rats of their MSSs even at the exaggerated exposure level used. These findings not only bolster the observations reported by RODGMAN (16) but also the conclusions reached by DOULL et al. (80), PASCHKE et al. (81), and GAWORSKI et al., 17-19,87) on the effect of added ingredients listed by DoulL et al. on the chemical and biological properties of cigarette MSS.

Over the years it has been repeatedly asserted [see (16)] that cigarette ingredients added at normal levels to pre-1980 cigarettes or at slightly increased levels to more recent lower "tar" cigarettes might adversely modify the chemistry and biology of the MSSs from such cigarettes. However, no chemical or biological evidence has been presented in support of such assertions. 


\section{INHIBITORS AND ANTICARCINOGENS IN CIGARETTE MSS}

In the preceding sections, a) the listing of numerous MSS components as significant toxicants is questioned and b) the assertions that ingredients added to cigarette tobacco adversely affect the chemical and biological properties of MSS are shown to be in error. In this section we will discuss the identified MSS components that have been shown in bioassays to significantly diminish the adverse biological effects of a number of the listed MSS toxicants.

The toxicological properties of a MSS component asserted to adversely affect the smoker have generally been defined in one or more bioassays devoted to the study of the effect of the component administered individually to a host. In most cases other than numerous studies of tumorigenesis, the effect on the toxicological property of a specific compound by other compounds such as those in the complex MSS aerosol has not been studied. The toxicological effect of a specific component in MSS is usually derived by extrapolation from the effect observed in one or more bioassays with the individual component.

It is known that the complex MSS aerosol has a significant effect on the chemistry of components in it. For example, a) the rate of conversion of $\mathrm{NO}$ to $\mathrm{NO}_{2}$ is significantly different in the MSS aerosol than in a system comprising only NO and $\mathrm{O}_{2}$ (95-97) and b) methyl nitrite reported as an MSS component is not formed during the smoking process but is formed during ageing of the MSS during the analytical procedure (98). If the chemistry of an MSS aerosol component be altered by the presence of thousands of other aerosol components, then logic dictates that its toxicology will also be altered.

Except for tumorigenic effects, little has been reported on the effect of other components in the complex MSS aerosol on the toxicological properties of an individual component. The tumorigenicity of many MSS components has been discussed frequently and in great detail but little has been written about the biological activity of nontumorigenic MSS components reported to counteract the tumorigenicity in laboratory animals of the various tumorigens.

In 1941, SHEAR and LEITER (99) described in detail the many factors affecting tumorigenicity of a chemical. In the mid-1940s, several nontumorigenic aromatic hydrocarbons (benzene, naphthalene, anthracene) administered with $\mathrm{B} a \mathrm{P}$ or dibenz $[a, h]$ anthracene (DBA) significantly diminished the $\mathrm{B} a \mathrm{P}$ and DBA tumorigenicity (100). In recent lists of MSS toxicants, benzene, $\mathrm{B} a \mathrm{P}$, and DBA are listed as significant tumorigens. Reported many times, however, is the noncarcinogenicity of benzene in the solvent-control group when it was used as the solvent for known or suspect tumorigens in skin-painting bioassays $(101,102)$.

STEINER and FALK (103) reported that benz $[a]$ anthracene $(\mathrm{B} a \mathrm{~A})$, categorized as either an extremely weak or an inactive mouse-skin tumorigen (104), significantly diminishes DBA tumorigenicity when both DBA and $\mathrm{B} a \mathrm{~A}$ are administered simultaneously by subcutaneous injection. Despite this and similar bioassay results plus the presence of $\mathrm{B} a \mathrm{~A}$ and DBA in MSS, both are repeatedly categorized as significant tumorigens in cigarette MSS! Similar inhibition was reported with mixtures of 7,12-dimethylbenz[a]anthracene (DMBA) and several inactive PAHs (105).
In subsequent studies, other nontumorigenic PAHs (phenanthrene, fluoranthene, pyrene) were reported to be effective antitumorigens against $\mathrm{B} a \mathrm{P}$ and $\mathrm{DMBA}(106,107)$. The nontumorigenic hydrocarbons - benzene, naphthalene, anthracene, phenanthrene, fluoranthene, pyrene - are MSS components, present at per cigarette delivery levels far in excess of those of $\mathrm{B} a \mathrm{P}, \mathrm{DBA}$, or any of the other PAHs classified as tobacco smoke toxicants.

Much evidence collected since 1932 on the tumorigenicity of PAHs indicates their tumorigenicity is not inherent but depends on specific metabolites that comprise one or more epoxides, dihydroxy compounds, and dihydroxy epoxides. For $\mathrm{B} a \mathrm{P}$, more than a dozen metabolites are known and they show a range of tumorigenicities (104).

Conversion of $\mathrm{B} a \mathrm{P}$ in an inhaled MSS particle to a particular metabolite cannot be a simple process. The more than 500 PAHs in cigarette MSS range from bicyclic to decacyclic structures. In a variety of chemical reactions, the rate of reaction decreases as the molecular weight (number of rings) of the PAH increases. That is, with stoichiometric levels of the PAH and the reactant, bicyclic PAHs react faster than tricyclic PAHs which in turn react faster than tetracyclic PAHs, etc.

Diol, epoxide, and/or diol-epoxide metabolites structurally similar to those described for $\mathrm{B} a \mathrm{P}$ have been reported for many PAHs, e.g., naphthalene, anthracene, phenanthrene, $\mathrm{B} a \mathrm{~A}$, benzo[c]phenanthrene, pyrene, chrysene, DBA, benzo $[b]$ triphenylene, and DMBA (104). All of these and structurally similar PAHs have been reported as cigarette MSS components (108).

In a situation, such as the formation of metabolites, where an equimolar mixture of bicyclic through hexacyclic PAHs is present, a pentacyclic aromatic hydrocarbon such as $\mathrm{B} a \mathrm{P}$ will form little of its metabolite(s) compared to the levels formed by a more reactive bicyclic or tricyclic aromatic hydrocarbon. Numerous in vitro studies have demonstrated that inclusion of equimolar quantities of lower molecular weight PAHs, such as phenanthrene or anthracene, inhibits the hydroxylation-epoxidation of $\mathrm{B} a \mathrm{P}$ in hepatic microsomes (109). However, PAH data from HoFFMANN and WYNDER (110) and RODGMAN and COOK (111) indicate the PAH classes (bicyclic, tricyclic, etc.) in MSS are present at significantly higher molar levels than the pentacyclic PAHs which include $\mathrm{B} a \mathrm{P}$ and DBA.

In an in vitro study, the nontumorigenic PAHs pyrene and fluoranthene significantly inhibited the binding of a tumorigenic PAH to calf thymus DNA (enzyme source $=$ mouse skin homogenate) (SLAGA and BouTWELL, 112; SLAGA et $a l ., 113)$. The in vitro inhibition of the hydroxylation reaction is paralleled by a reduction of in vivo tumorigenicity.

Because of their vapor pressure properties, tumorigenic PAHs (BaP, DBA, etc.) and aza-arenes are present primarily in the MSS particulate phase. Similarly, many of the reported anticarcinogens or inhibitors occur in the MSS particulate phase $(9,42,114)$, e.g., high molecular weight alkanes (115), $\beta$-sitosterol and cholesterol (115), $\alpha$-tocopherol (116), indole (117), indole-3-acetonitrile (118), duvatrienediols (119), and PAHs (anthracene, phenanthrene, pyrene, fluoranthene, $\mathrm{B} e \mathrm{P}$ ) [see (9)].

Despite the fact that the anticarcinogenicity of certain components of tobacco (120) and tobacco smoke $(121,122)$ 
and of tobacco smoke itself (121) has been known for over four decades, most discussions are directed at them as toxicants. Seldom is any significant discussion directed at smoke components known to possess anticarcinogenic properties. In a brief 1964 review of the possibility of anticarcinogenic agents in tobacco smoke, WYNDER and HOFFMANN [see pages 296, 330 in (123)] discussed the findings of STEINER and FALK (103) and KOTIN and FALK (124) in their studies with potent and weakly tumorigenic PAHs in the subcutaneous injection bioassay as well as their own findings in the mouse skin-painting bioassay $(125,126)$. Ignored was the discussion by KOTIN and FALK (124) on the anticarcinogenicity vs. $\mathrm{B} a \mathrm{P}$ or vs. DBA of nine PAHs (anthracene, benzo[ $a]$ fluorene, $\mathrm{B} a \mathrm{~A}$, chrysene, pyrene, $\mathrm{B} e \mathrm{P}$, benzo[k]fluoranthene, benzo[ghi]fluoranthene, perylene), two aza-arenes (benzo[a]carbazole, benz $[c]$ acridine), and 2-naphthol. All but the two azaarenes had been identified in cigarette MSS prior to their 1964 review. Subsequently, the aza-arenes noted were identified as MSS components $(127,128)$.

Earlier,WYNDER and HOFFMANN (129) had reported on MSS components that inhibited the action of a "tumorigen" invariably listed as significant. The finding was an outgrowth of their investigation of the effect of organic solvent extraction of tobacco on the PAH content of MSS. Cigarettes fabricated from the extracted tobacco yielded lower quantities of $\mathrm{B} a \mathrm{P}$ and DBA in MSS $(34,130)$. Skinpainting bioassays with MS CSCs from the control and extracted tobaccos gave a lower percentage of tumorbearing animals (\% TBA) in the group treated with the extracted tobacco CSC. However, the decrease in \% TBA was considerably less than the percent decrease in the level of tumorigenic PAHs in the CSC (131). One explanation for the difference was that the solvent extracted almost all the alkanes from the tobacco. Thus, the alkanes were absent from the MSS from extracted-tobacco cigarettes. This fraction (constituting about $3 \%$ of MS CSC) was reported to significantly inhibit the tumorigenicity of $\mathrm{B} a \mathrm{P}$ $(126,129,132)$.

Mouse skin-painting studies with $\mathrm{B} a \mathrm{P}$ and the alkanes $n$ hentriacontane and $n$-pentatriacontane showed they significantly inhibit $\mathrm{B} a \mathrm{P}$ tumorigenicity $(126,129,132)$. The MSS of a cigarette delivering $20 \mathrm{mg}$ of CSC contains about 0.6 $\mathrm{mg}(600000 \mathrm{ng})$ of the alkane fraction and $10 \mathrm{ng}$ of $\mathrm{B} a \mathrm{P}$, an alkane fraction: $\mathrm{B} a \mathrm{P}$ ratio of $60000: 1$, far in excess of the ratios that produced significant inhibition of $\mathrm{B} a \mathrm{P}$ tumorigenicity (WYNDER and HOFFMANN, 57,123,129).

WYNDER and HOFFMANN [see pp. 245-247, 628 in (57)] again discussed anticarcinogenic components of tobacco smoke:

Any discussion of as complex a carcinogen as tobacco smoke should at least mention the existence of anticarcinogens. These are substances that reduce or "neutralize" the effect of a carcinogen by reacting with the carcinogen or a carcinogenic metabolite, thereby deactivating it, or by competing for reaction with cell constituents, or by interfering with the resorption of a carcinogen ...

The existence of anticarcinogens, however, must be considered in evaluating any complex mixture such as tobacco smoke condensate...

An explanation of the tumorigenic activity of tobacco smoke condensate in terms of single constituents is made more difficult by the presence of substances that may act as anticarcinogens and/or absorption retarders, especially for tumorigenic agents. It is known that structurally related noncarcinogenic hydrocarbons can inhibit the effect of carcinogenic hydrocarbons ...

Several investigators have noticed some inhibition of tumor growth by tobacco smoke condensate ... [including] HoFFMAN and GRIFFIN [122] . . FALK et al. [120] . . [and] HOMBURGER and TREGIER [sic] [133] . . it should not come as a surprise that a material which has been proved to be carcinogenic may also interfere with tumor development, if not with tumor initiation ...

They also noted [see pp. 370-371, 628-629 in (57)]:

An explanation of the tumorigenic activity of tobacco smoke condensate in terms of single constituents is made more difficult by the presence of substances that may act as anticarcinogens and/or absorption retarders, especially for tumorigenic agents. It is known that structurally related noncarcinogenic hydrocarbons can inhibit the effect of carcinogenic hydrocarbons. The same interrelationship may apply to tumor-promoting and nontumor-promoting phenols.

Numerous compounds demonstrated in various bioassays to be highly effective anticarcinogens against many MSS toxicants have been identified in tobacco smoke at per cigarette delivery levels far in excess of those of the alleged tumorigens. Seldom have these anticarcinogenic MSS components been discussed in the numerous reviews of the biological properties of MSS. Even though some of the earliest data on MSS components, e.g., the alkanes, that inhibit $\mathrm{B} a \mathrm{P}$ tumorigenicity in the skin-painting bioassay were provided by WYNDER and HOFFMANN [see pages 370-371, 628-629 (57), (126)], they more often preferred to discuss alkanes as major precursors of tumorigenic PAHs in MSS [(see pp. 496-501 in (57), (110), (126), (134)] rather than inhibitors of $\mathrm{B} a \mathrm{P}$ tumorigenicity. MSS components reported to possess significant inhibitory or anticarcinogenic action against various tumorigenic PAHs and NNAs in MSS have been cataloged $(9,42,114)$.

Those opposed to cigarette smoking view the complex mixture MSS differently from other complex mixtures such as raw or cooked foods, gasoline and diesel engine exhausts, factory effluents, etc. [see $(135,136)]$. Most are reluctant to accept the premise that a nontumorigenic component will offset the tumorigenicity of a tumorigen in animals treated with the complex mixtures CSC, MSS, SSS, or environmental tobacco smoke (ETS) containing the two (137). Other MSS components may have also influenced the mouse skin-painting results obtained with control tobacco and extracted tobacco CSCs. Hexane extraction of tobacco not only removes alkane inhibitors thus making impossible their transfer to MSS but also removes substantial amounts of $\beta$-sitosterol (138), $\alpha$-tocopherol $(116,139)$, indole (117), duvatrienediols $(119,140)$, and $D$-limonene $(141,142)$, thus eliminating or drastically reducing their transfer to MSS during smoking. Subsequently, it was demonstrated that: a) These smoke components are present by transfer from tobacco to MSS during smoking and to SSS during smolder between puffs or they are generated during smoking. b) The compounds listed are anticarcinogenic vs several of the listed tumorigens, e.g., PAHs, NNAs, ethyl carbamate. However, in the 1950s, neither the identity of several of these tobacco or smoke components nor their anticarcinogenicity was known.

Comparison of identified MSS components (4) with lists of compounds $(135,143)$ that possess inhibitory or anti- 
carcinogenic action in tumorigenesis studies reveals not only that MSS contains many anticarcinogens but also that their MSS levels usually exceed those of the components listed as significant tumorigens. Previously we discussed a few inhibitory and anticarcinogenic MSS components, but they represent a small sample of the MSS components reported to exhibit such properties. From the review by SLAGA and DigIOVANNI (135) and other reports (143), we compiled a list of MSS components reported to counteract the tumorigenicity of MSS toxicants (Table 11).

From the per cigarette MSS deliveries (Table 11), it may be calculated that the tumorigenic PAHs listed contribute from 4 to $10 \mu \mathrm{g} / \mathrm{g}$ of MS CSC. Nontumorigenic PAHs (naphthalene, anthracene, pyrene, phenanthrene, fluoranthene, benzo[e]pyrene, benzo[b]triphenylene) total 90 to $180 \mu \mathrm{g} / \mathrm{g}$ of CSC. The anticarcinogenic effect of nontumorigenic PAHs and weakly tumorigenic or nontumorigenic aza-arenes vs. carcinogenic PAHs has been known since the 1940s (103,135).

An interesting aspect of Table 11 is that it includes the dioxins as antitumorigens. SLAGA and DigiovanNI (135) summarized the studies in which dioxins were shown to interfere with the enzyme pathways responsible for tumorigenesis of several of the most potent PAHs. The dioxins were not listed as MSS toxicants in previous tabulations similar to Table $11(9,42,114)$. In fact, only one toxicant list issued since 1990 (33) has included the dioxins even though their presence in MSS was known in 1980 (60). Is the omission of such MSS toxicants related in any way to the fact that dioxins are significant antitumorigens vs. some of the most potent mouse-skin tumorigenic PAHs present in MSS? The 1964 Advisory Committee in Chapter 6 of its 1964 Report mentions that 27 nontumorigenic PAHs had been identified in MSS, but none by name [see Chapt. 6, p. 55 in (6)]. Was the omission of their identities related to the fact that several were known to be antitumorigenic to several potent mouse-skin tumorigens such as $\mathrm{B} a \mathrm{P}$ ?

\section{ANTIMUTAGENS IN CIGARETTE MSS}

In a review of antimutagens and inhibitors of mutagenesis, RAMEL et al. (162) discussed the many antimutagens found naturally occurring in plants. They did not discuss tobacco but did discuss the natural occurrence of the following antimutagens: $\alpha$-tocopherol, $2 \mathrm{H}$-1-benzopyran-2-one, 7 hydroxy-2H-1-benzopyran-2-one, and 3-phenyl-2-propenal. All four have been identified in tobacco; all but 7-hydroxy2H-1-benzopyran-2-one have been found in MSS.

LEE and REED (163) investigated the antimutagenicity of nicotine vs. $N$-nitrosodimethylamine (NDMA) and nicotine vs. $\mathrm{B} a \mathrm{P}$ in the Ames test (Salmonella typhimurium TA 100). They observed that nicotine inhibits the mutagenicity of NDMA but not of $\mathrm{B} a \mathrm{P}$. Although the mechanism of this antimutagenicity was not elucidated, the more recent report by MURPHY and HEILBRUN (164) on the inhibition of NNN metabolism by nicotine suggests nicotine inhibition of NNA activation may be involved. LEE et al. (157) repeated the earlier experiment and not only confirmed the antimutagenic effect of nicotine on NDMA but also the similar activity of nornicotine and cotinine. Recently BROWN et al. (158) reported the antimutagenicity of nicotine and cotinine vs. 4-(methylnitrosamino)-1-(3-pyridyl)-1-butanol (NNAL). LEE et al. (165) reported that CSC inhibits the mutagenic activity of several $N$-heterocyclic amines when tested in the Ames assay with Salmonella typhimurium TA 98 in the presence of the S-9 activation system. The mutagenic $N$ heterocyclic amines tested included Glu-P-1, Glu-P-2, TrpP-1, Trp-P-2, IQ, and MeIQ. These compounds are among the most potent mutagens known (166-169). Several have also been reported to be tumorigenic in mammalian bioassays (170). In one of the first demonstrations of antimutagens in tobacco smoke, LEE et al. (165) reported that 50 to $100 \mu \mathrm{g}$ of CSC per plate suppresses the mutagenic activity of these compounds by as much as $80 \%$. Enzymatic studies indicate that $\mathrm{CSC}$ is a potent inhibitor of cytochrome P-450 dependent monooxygenase. Therefore, it appears that CSC exerts its antimutagenicity by inhibiting the P-450 system. LEE et al. (165) subsequently reported that fractionation of CSC yields fractions that show low mutagenicity themselves but are significantly antimutagenic.

Only a few of the listed MSS tumorigens have ever been tested for tumorigenicity to lung tissue by exposure of animals via inhalation. The results with all but one of the four MSS components (BaP, $N$-nitrosodimethylamine, $N$-nitrosodiethylamine, polonium-210), tested via inhalation at dose levels substantially exceeding those in MSS, were rated "equivocal" (171). Only polonium-210, administered via inhalation at massive dose levels to rats, produced squamous cell carcinoma, the lung tumor type similar to that associated statistically with cigarette smoking. However, the SuRgeON GENERAL $(43,172)$ and HoFFMANN and HECHT (7) discounted the effect of polonium-210 in MSS in lung-cancer causation in active smokers. From the type of evidence available presently, it is doubtful that many of the toxicants should be included in the various lists. Examination of data and reports on the tobacco smoke components present in one or more of the many lists sustains the premise that it is inappropriate to use such lists as evidence of any relationship between exposure to MSS and lung cancer induction in smokers or exposure to ETS and lung cancer induction in nonsmokers.

Several specific components could and should be excluded from the toxicant lists for reasons other than the failure to induce lung tumors via inhalation. a) By the early 1960s, dibenzo[ $[a, l]$ pyrene had been reported in MSS by several groups [see account in (34)]. For its identification, the investigators relied on a published UV spectrum purportedly that of synthetic dibenzo[ $a, l]$ pyrene (dibenzo[def,p]chrysene). However, in 1966 it was demonstrated that the published spectrum was that of an isomer, dibenz[a,e]aceanthrylene (dibenzo[a,e]fluoranthene) (173). b) Previously we noted the failure by many research groups between 1963 and 2000 to confirm the presence in MSS of the tumorigenic aza-arenes reported by VAN DUUREN et al. (55). Dibenz $[a, j]$ acridine was reported recently by RUSTEMEIER et al. (21). c) The precursors of arsenic and NDELA in MSS have been banned from US tobacco agronomy since 1952 and 1981, respectively. 
Table 11. Inhibitors, anticarcinogens, and antimutagens in tobacco smoke ${ }^{\text {a }}$

\begin{tabular}{|c|c|c|c|c|c|}
\hline Component & CAS no. & $\begin{array}{l}\text { Approx. delivery } \\
\mu \mathrm{g} / \mathrm{g} \text { MS CSC }\end{array}$ & $\begin{array}{c}\text { Effective } \\
\text { against }\end{array}$ & AT, AM $^{\mathrm{b}}$ & References $^{c}$ \\
\hline \multicolumn{6}{|c|}{ Hydrocarbons, aliphatic } \\
\hline $\begin{array}{l}\text { Saturated aliphatic hydrocarbons }{ }^{d} \\
\text { e.g., } \mathrm{C}_{31} \mathrm{H}_{64} \\
\mathrm{C}_{35} \mathrm{H}_{72}\end{array}$ & $\begin{array}{l}630-04-6 \\
630-07-9\end{array}$ & $\begin{array}{l}30000 \\
{[2500]^{e}}\end{array}$ & $\mathrm{BaP}$ & AT & Wynder and Hoffmann (126) \\
\hline \multirow[t]{2}{*}{$D$-Limonene } & $5989-27-5$ & $15-50$ & NNK & AT & Wattenberg and Coccia (144) \\
\hline & & & $\mathrm{DB}[a,] \mathrm{P}$ & AT & Homburger et al. (145) \\
\hline \multicolumn{6}{|c|}{ Hydrocarbons, aromatic } \\
\hline Benzene & $71-43-2$ & 480-1900 & $\mathrm{BaP}, \mathrm{DBA}$ & AT & Crabtree (100) \\
\hline Naphthalene & $91-20-3$ & $80-160$ & $\mathrm{BaP}, \mathrm{DBA}$ & AT & Crabtree (100) \\
\hline Anthracene & $120-12-7$ & 4-7 & $\mathrm{BaP}, \mathrm{DBA}$ & AT & Crabtree (100) \\
\hline Phenanthrene & 85-01-8 & $2-4$ & DMBA & AT & DiGiovanni et al. $(107)^{c}$ \\
\hline Fluoranthene & $206-44-0$ & $3-4$ & DMBA & AT & $\begin{array}{l}\text { DiGiovanni et al. }(107)^{c} \\
\quad \text { Slaga et al. }(106)^{c}\end{array}$ \\
\hline Pyrene & $129-00-0$ & $3-4$ & DMBA & AT & $\begin{array}{l}\text { DiGiovanni et al. }(107)^{\mathrm{c}} \text {; } \\
\quad \text { Slaga et al. }(106)^{\mathrm{c}}\end{array}$ \\
\hline Benz[a]anthracene & $56-55-3$ & $0.8-2.8$ & DBA & AT & Steiner and Falk (103) \\
\hline Benzo[e]pyrene & $192-97-2$ & 0.2 & DMBA & AT & $\begin{array}{l}\text { DiGiovanni et al. }(107)^{c} \\
\quad \text { Slaga et al. }(106)^{c}\end{array}$ \\
\hline Benzo $[b]$ triphenylene ${ }^{f}$ & $215-58-7$ & 0.05 & $\begin{array}{l}\text { MC, DBA, } \\
\text { DMBA }\end{array}$ & AT & $\begin{array}{l}\text { Slaga and Boutwell (112) } \\
\text { Slaga et al. }(106)^{c}\end{array}$ \\
\hline \multicolumn{6}{|c|}{ Alcohols } \\
\hline Ethanol & $64-17-5$ & & NNN & AT & Waddell and Marlowe ${ }^{c}(146)$ \\
\hline & & & NNN & AM & Farinati et al. (147) \\
\hline 1-Butanol & $71-36-3$ & & NNN & AT & Waddell and Marlowe ${ }^{\dagger}(146)$ \\
\hline 2-Propanol, 2-methyl- $\{$ tert-butanol $\}$ & $75-65-0$ & & NNN & AT & Waddell and Marlowe ${ }^{f}(146)$ \\
\hline $\begin{array}{l}\alpha-4,8,13 \text {-Cyclodecatriene-1,3-diol, } \\
\text { 1,5,9-trimethyl-12- (1-methylethyl)- } \\
\{\alpha-4,8,13 \text {-duvane-1,3-diol }\}\end{array}$ & $57605-80-8$ & $8-20$ & DMBA & AT & Saito et al. ${ }^{c}(140)$ \\
\hline $\begin{array}{l}\beta-4,8,13 \text {-Cyclodecatriene-1,3-diol, } \\
\text { 1,5,9-trimethyl-12- (1-methylethyl)- } \\
\{\beta-4,8,13 \text {-duvane-1,3-diol }\}\end{array}$ & $57605-81-9$ & $12-25$ & DMBA & AT & Saito et al. ${ }^{c}(140)$ \\
\hline$\beta$-Sitosterol & $83-46-5$ & $400-550$ & $\begin{array}{l}\text { NNA } \\
\text { PAH }\end{array}$ & AT & $\begin{array}{l}\text { Wattenberg }{ }^{\mathrm{c}}(148) \\
\text { Yasukawa et al. }^{\mathrm{c}}\end{array}$ \\
\hline \multirow[t]{2}{*}{ Cholesterol } & $57-88-5$ & $120-240$ & NNA & AT & Cohen et al. ${ }^{c}$ \\
\hline & & Acids & & & \\
\hline $\begin{array}{l}\text { Acids, long-chained aliphatic } \\
\text { e.g., } \mathrm{C}_{16} \mathrm{H}_{32} \mathrm{O}_{2} \\
\mathrm{C}_{18} \mathrm{H}_{36} \mathrm{O}_{2}\end{array}$ & $\begin{array}{l}57-10-3 \\
57-11-4\end{array}$ & & NNA & AM & Takeda et al. (149) \\
\hline Benzoic acid, 3,4,5-trihydroxy-\{gallic acid\} & $149-91-7$ & & NNA & AT & Mirvish et al. ${ }^{c}$ \\
\hline $\begin{array}{l}\text { 1-Propene-1,2,3-tricarboxylic acid } \\
\quad \text { \{aconitic acid }\}\end{array}$ & $499-12-7$ & & $\mathrm{BaP}$ & AT & $\begin{array}{l}\text { Kallistratos } \\
\text { Fasske } \\
\end{array}$ \\
\hline $\begin{array}{l}\text { 2-Propenoic acid, 3-(3,4-dihydroxyphenyl)- } \\
\text { \{cinnamic acid, 3,4-dihydroxy-\} } \\
\text { \{caffeic acid\} }\end{array}$ & $331-39-5$ & & $\mathrm{BaP}$ & AT & Wattenberg et al. ${ }^{c}$ \\
\hline \multicolumn{2}{|c|}{$\begin{array}{l}\text { 2-Propenoic acid, 3-(3-hydroxy-4-methoxyphenyl)- } \\
\text { \{cinnamic acid, 3-hydroxy-4-methoxy-\} }\end{array}$} & & $\mathrm{BaP}$ & AT & Wattenberg (148) \\
\hline $\begin{array}{l}\text { 2-Propenoic acid, 3-(2-hydroxyphenyl)- } \\
\text { \{cinnamic acid, 2-hydroxy-\} }\end{array}$ & $614-60-8$ & & $\mathrm{BaP}$ & AT & Wattenberg et al. ${ }^{\dagger}$ \\
\hline $\begin{array}{l}\text { 2-Propenoic, 3-phenyl- } \\
\quad\{\text { cinnamic acid\} }\end{array}$ & $621-82-9$ & & NPYR, NNN & AT & Chung et al. $(150,151)$ \\
\hline \multirow[b]{2}{*}{ Phenol } & & Phenols & & & \\
\hline & $108-95-2$ & $1000-7000$ & $\begin{array}{l}\text { BaP } \\
\text { NNN, NPYR }\end{array}$ & AT & $\begin{array}{l}\text { Van Duuren et al. }(152) \\
\text { Chung et al. }(150,151)\end{array}$ \\
\hline Phenol, 4-methoxy- & $150-76-5$ & & $\mathrm{BaP}$ & AT & Wattenberg et al. ${ }^{\dagger}$ \\
\hline \multirow[t]{3}{*}{$\alpha$-Tocopherol $\{$ vitamin E\} } & $59-02-9$ & $400-600$ & $\begin{array}{l}\text { MC, DMBA, } \\
\text { DB[a,j]P, } \\
\text { 1,2-DMH }\end{array}$ & AT & $\begin{array}{l}\text { Shamberger }{ }^{c} \text {; Shklar }{ }^{c} \text {; Slaga } \\
\text { and Bracken }{ }^{c} ; \text { Viaje et al. }{ }^{c} \text {; } \\
\text { Weerapradist and Shklar }^{c}\end{array}$ \\
\hline & & & NNA & AT & Thompson (153) \\
\hline & & & csc & AM & $\operatorname{Rosin}^{c}$ \\
\hline $\begin{array}{l}\text { 2H-1-Benzopyran-2-one, 6,7-dihydroxy- } \\
\quad \text { esculetin\} }\end{array}$ & $305-01-1$ & & NNK & AT & Teel and Castonguay (154) \\
\hline
\end{tabular}




\begin{tabular}{|c|c|c|c|c|c|}
\hline Component & CAS no. & $\begin{array}{l}\text { Approx. delivery } \\
\mu \mathrm{g} / \mathrm{g} \text { MS CSC }\end{array}$ & $\begin{array}{l}\text { Effective } \\
\text { against }\end{array}$ & $\mathrm{AT}, \mathrm{AM}^{\mathrm{b}}$ & References $^{c}$ \\
\hline \multicolumn{6}{|c|}{$\mathrm{N}$-Containing components } \\
\hline \multirow[t]{3}{*}{ Indole } & $120-72-9$ & $400-600$ & NNA & AT & Matsumoto et al. ${ }^{c}$ \\
\hline & & & NNN, NPYR & & Chung et al. $(150,151)$ \\
\hline & & & NNK & & Chung et al. (155) \\
\hline Indole-3-acetonitrile & $771-51-7$ & & $\mathrm{BaP}$ & AT & Kovacs and Somogyi ${ }^{c}$ \\
\hline $\begin{array}{l}\text { 1H-Purine-2,6-dione, 3,7-dihydro-3,7- } \\
\text { dimethyl- }\{\text { theobromine }\end{array}$ & $83-67-0$ & & EC & AT & Nomura $^{c}$ \\
\hline $\begin{array}{l}\text { 1H-Purine-2,6-dione, 3,7-dihydro-1,3,7- } \\
\text { trimethyl- \{caffeine\} }\end{array}$ & $58-08-2$ & & $\begin{array}{l}\text { EC, DMB, } \\
\text { NNA }\end{array}$ & AT & $\begin{array}{l}\text { Nomura }{ }^{\text {c; }} \text { Perchellet and } \\
\text { Boutwell }{ }^{\text {c; }} \text { Mirvish et al. }{ }^{c}\end{array}$ \\
\hline \multirow[t]{3}{*}{ Nicotine } & $54-11-5$ & & NNK & AT & Schüller et al. (156) \\
\hline & & & NDMA & AM & Lee et al. (157) \\
\hline & & & NNAL & AM & Brown et al. (158) \\
\hline \multirow[t]{2}{*}{ Nornicotine } & $494-97-3$ & & NDMA & AM & Lee et al. (157) \\
\hline & & & NNAL & AM & Brown et al. (158) \\
\hline \multirow[t]{2}{*}{ Cotinine } & $486-56-6$ & & NDMA & AM & Lee et al. (157) \\
\hline & & & NNAL & AM & Brown et al. (158) \\
\hline \multicolumn{6}{|c|}{ Miscellaneous components } \\
\hline 2H-Benzopyran-2-one \{coumarin\} & $91-64-5$ & & $\mathrm{BaP}, \mathrm{DMBA}$ & AT & Wattenberg et al. ${ }^{\mathrm{c}}$ \\
\hline $\begin{array}{l}3 H \text {-2-Furanone, dihydro-5-methyl- } \\
\{\alpha \text {-angelica lactone }\}\end{array}$ & $108-29-2$ & & $\mathrm{BaP}$ & AT & Wattenberg et al. ${ }^{c}$ \\
\hline $\begin{array}{l}\text { Benzoic acid, 3,4,5-trihydroxy-, propyl ester }{ }^{d} \\
\quad \text { ppropyl gallate\} }\end{array}$ & $121-79-4$ & & NNK & AT & $\begin{array}{l}\text { Lo and Stich }{ }^{c} \text {; Teel and } \\
\text { Castonguay (154) }\end{array}$ \\
\hline Dioxin & & & $\begin{array}{c}\text { DMBA, MC, } \\
\text { BaP, 7-MBA, } \\
\text { 12-MBA, } \\
\text { 5-MeC, DBA }\end{array}$ & AT & $\begin{array}{l}\text { Berry et al. (159); Cohen et al. } \\
\text { (160); DiGiovanni et al. (161) }\end{array}$ \\
\hline Carbon disulfide & $75-15-0$ & & 1,2-DMH & AT & Wattenberg and Fiala ${ }^{c}$ \\
\hline Maleic anhydride & $108-31-6$ & & PAH, DMBA & AT & Klein ${ }^{c} ;$ Slaga et al. ${ }^{c}$ \\
\hline \multirow[t]{2}{*}{ Selenium } & $7782-49-2$ & & DMBA & AT & Shamberger ${ }^{c}$ \\
\hline & & & NNA & AT & Thompson (153) \\
\hline Cysteine & $52-90-4$ & & NDMA & AT & Lo and Stich ${ }^{c}$ \\
\hline
\end{tabular}

${ }^{a}$ Abbreviations: $\mathrm{BaP}=$ benzo[a]pyrene; $\mathrm{DBA}=$ dibenz $[a, h]$ anthracene; $\mathrm{DB}[a, \lambda \mathrm{P}=$ dibenzo[a,i]pyrene, = benzo[rsf $]$ pentaphene; DMBA = 7,12-dimethylbenz[a]anthracene; 1,2-DMH = 1,2-dimethylhydrazine; 7-MBA = 7-methylbenz[a]anthracene; 12-MBA = 12-methylbenz[a]anthracene; 5-MeC = 5-methylchrysene; EC = ethyl carbamate; $M C=3$-methylcholanthrene, = 1,2-dihydro-3-methylbenz[]]aceanthrylene; NDMA = N-nitrosodimethylamine; NNA = N-nitrosamine; NNAL = 4-(methylnitrosamino)-1-(3-pyridyl)-1-butanol;

$\mathrm{NNN}=N$-nitrosonornicotine; NNK = 4-(N-methylnitrosamino)-1-(3-pyridinyl)-1-butanone; NPYR = $N$-nitrosopyrrolidine; PAH = polycyclic aromatic hydrocarbon.

${ }^{\mathrm{b}} \mathrm{AT}=$ test for antitumorigenicity; $\mathrm{AM}=$ test for antimutagenicity

${ }^{c}$ Representative references to inhibition, anticarcinogenicity, and/or antimutagenicity. Details of this reference may be found in Fay et al. (143) and/or Rodgman (42). Additional references may be found in $(42,107,135,143)$.

${ }^{d}$ This fraction consists primarily of the normal-, iso- (2-methyl-), and anteiso- (3-methyl-) alkanes from $\mathrm{C}_{15}$ to $\mathrm{C}_{40}$.

${ }^{\mathrm{e}}$ Average weight ( $\mathrm{\mu g} / \mathrm{g}$ MS CSC) of each hydrocarbon isomer.

'Benzo[b]triphenylene was formerly known as dibenz[a,c]anthracene.

\section{THE COMPENSATION ASSERTION}

Because of the lack of derogatory evidence from the anti-tobacco critics about added ingredients and the gradual accumulation of evidence that the usual or increased levels of added ingredients produced no significant adverse effect on the chemical and biological properties of the MSS, criticism was shifted from the added ingredient issue to compensation, i.e., the smoker is taking more puffs, larger puffs, and more particularly, blocking the filter-tip ventilation holes of the cigarette to compensate personally for the lower "tar" and nicotine deliveries as measured in the FTC procedure. The compensation issue and discussions of it have become so massive that the issue is much too detailed to be dealt with at length in our review. Much of the issue has been put in perspective by SCHERER (174) and by BAKER and LEWIS (175).
19 THE RISE AND FALL OF THE MAJOR CIGARETTE MSS TOXICANTS: EXCEPTION - THE TOBACCOSPECIFIC $N$-NITROSAMINES (TSNAs)

Since the mid-1950s various MSS toxicants, either as an individual component or a class of components, have had their moment of publicity but one by one their importance gradually faded. Chronologically, the first toxicants to become infamous were the tumorigenic PAHs with $\mathrm{B} a \mathrm{P}$ at the pinnacle because of its potent tumorigenicity to mouse skin and its level in cigarette MSS. The chronological sequence of the rise to notoriety of the various individual and/or class of toxicants has been previously depicted [see Figure 1 in RoDGMAN et al. (176)] but the depiction does not show when the prominence of most of them declined. 
In the mouse skin-painting bioassay, neither $\mathrm{B} a \mathrm{P}$ nor the total tumorigenic PAHs account for the observed specific tumorigenicity (177). The $\mathrm{B} a \mathrm{P}$ content of CSC accounts for less than $2.5 \%$ and the total tumorigenic PAH content of CSC accounts for less than $3.5 \%$ of the CSC specific tumorigenicity [see Chapt. 6, p. 55 in (6), see p. 626 in (57), (178)]. Inclusion of tumorigenic aza-arenes reported by VAN DUUREN et al. (55) does not improve the situation. HOFFMANN and WYNDER (110) reported that doubling or tripling the level of 17 tumorigenic PAHs in CSC significantly increases the \% TBA (tumor-bearing animals) whereas others reported that a 10-fold (179) or 30-fold (180) increase in the $\mathrm{B} a \mathrm{P}$ level in CSC produces no change in the \% TBA. In the early 1960s, the promoting effect of the MSS phenols on tumorigenic PAHs was advanced to explain the tumorigenic response observed in CSC-painted mice. Inclusion of this effect in the assessment accounted for about $5 \%$ of the $\%$ TBA. In addition, reports of no change in the tumorigenicity of CSC when significant amounts (75-90\%) of the phenols were removed from MSS (and the CSC) by selective filtration [see p. 626 in (57), (181)] and the inhibition of the specific tumorigenicity of $\mathrm{B} a \mathrm{P}$ by phenol (152) diminished the alleged importance of the promoting effect of phenols.

To offset the decrease in importance of the PAHs, azaarenes, and phenols, ciliastatic components in MSS then became the in-vogue toxicants. It was asserted, based on studies with clam cilia and mammalian ciliated tissue, that certain MSS toxicants impair lung ciliary activity thus preventing removal of tumorigen-containing smoke particles from the lung [see references in KENSLER and BATTISTA (182)]. Chief MSS ciliastats are formaldehyde, acetaldehyde, acrolein, $\mathrm{HCN}$, formic and acetic acids, and phenol. However, after 1968, the ciliary assertion faded with the demonstration that less than a third of the ciliastats reach the lung cilia in human smokers (183).

In the mid-1960s, several other MSS toxicants had their brief moment of infamy, e.g., ${ }^{210} \mathrm{Po}, \mathrm{NO}_{2}, \mathrm{CO}$. In their comparison of lung cancer incidence in uranium miners exposed to ${ }^{210} \mathrm{Po}$ vs. cigarette smokers exposed to MSS ${ }^{210} \mathrm{Po}$, HARLEY et al. (77) questioned the significance of ${ }^{210} \mathrm{Po}$ in tobacco-induced lung cancer. Concern over $\mathrm{NO}_{2}$ diminished with the demonstration that over $95 \%$ of the $\mathrm{NO}_{\mathrm{x}}$ in MSS is $\mathrm{NO}$, not $\mathrm{NO}_{2}$, and the conversion of $\mathrm{NO}$ to $\mathrm{NO}_{2}$ is impeded by other MSS components (95).

In the early $1960 \mathrm{~s}$, the formation of $N$-nitrosamines (NNAs) during tobacco smoking was suggested (184) as well as the possible presence of $N^{\prime}$-nitrosonornicotine (NNN) and $N^{\prime}$ nitrosoanabasine (NAB) in MSS (185). Between 1964 and the early 1970s, several volatile NNAs were identified in MSS. It was also determined that $60 \%$ to $85 \%$ of the volatile NNAs, like the phenols, are selectively filtered from MSS. The identification of several TSNAs, including NNN and $\mathrm{NAB}$, then followed.

Why have TSNAs maintained their status as important MSS toxicants while the importance of other individual and/or classes of toxicants has faded? Alternate exposures are possible with other toxicant classes including NNAs other than the TSNAs but, as their classification denotes, the TSNAs are "tobacco-specific". In the detailed 1984 outline of chemical carcinogenesis edited by SEARLE (186), the only class of MSS tumorigens discussed in 22 chapters comprising nearly 1400 pages is the NNAs!
Since the early 1960s, a "less hazardous" cigarette has been defined on the basis of three criteria [see p. iii, Report No. 1 in (35); p. 372 in (123); p. 503, 531 in (57)]: 1) the per cigarette delivery of a specific toxicant has been lowered, 2) the ratio of the specific toxicant to MSS "tar" has been lowered, and 3) the specific tumorigenicity of the MSS "tar" as measured in the mouse skin-painting bioassay has been lowered.

From bioassay results of more than 330 NNAs plus knowledge of fewer than 50 specific NNAs in MSS, it is obvious that the MSS NNAs cannot meet criterion 3). Over $330 \mathrm{~N}$ nitroso compounds variously administered to 40 different species have been reported as tumorigenic. No laboratory species is resistant to NNAs. In their summary of the results from $323 N$-nitroso compounds bioassayed from 1956 to 1984, PREUSSMANN and STEWART (187) reported that $87 \%$ of the $N$-nitroso compounds are tumorigenic. Over $70 \%$ of the $N$-nitroso compounds studied were NNAs; the remainder was $N$-nitrosamides.

Administration of most NNAs to laboratory animals via skin painting seldom results in carcinoma induction at the application site. Generally, tumors develop at site(s) remote from the painting site and various organs may be involved. This major difference between PAH and NNA tumorigenicity led to defining NNAs as organ-specific tumorigens. Failure to produce tumors with NNAs at the painting site subsequently led to studies of NNAs administered by alternate routes [injection (subcutaneous, intravenous, intraperitoneal), per $o s$, intratracheal instillation, etc.]. Administration of NNAs by inhalation was studied infrequently.

Skin-painting studies with six NNAs ( $N$-nitrosobutylmethylamine, $N$-nitrosodiethylamine, NDELA, NNK, $\mathrm{NNN}$ ) present in tobacco and/or tobacco smoke were reported by BRUNE and HENNING (188), HOFFMANN and GRAFFI (189), HERROLD (190), the IARC (38), HOFFMANN et al. (191), and LAVOIE et al. (192). Tumors developed elsewhere in the test animals but none at the painting site. In a painting study by DEUTSCH-WENZEL et al. (193), NNN induced a few skin tumors, but no dose-response relationship was observed over a $12.5-$ to $200-\mu$ g range. In the same experiment, the tumorigenic potency to skin of $N$ nitroso- $N$-methylurea was estimated to be about $4 \%$ of that of $\mathrm{B} a \mathrm{P}$ (193). In painting studies with $N$-nitroso- $N$-alkylureas, tumors did develop at the skin-painting site, but to date, no $N$-nitroso- $N$-alkylurea has been identified in tobacco or its smoke.

\section{THE ARTIFACTUAL FORMATION OF $N$-NITROSAMINES}

In 1964, NEURATH et al. (194) reported $N$-nitroso- $n$-butylmethylamine and two unidentified NNAs in MSS. The next year, NEURATH et al. (195) discounted their reported findings because of artifactual formation of the NNAs during their collection/analytical procedure. However, with a modified analytical and collection procedure, $N$-nitrosodimethylamine (4 ng/cig) and $N$-nitrosopyrrolidine (4 ng/cig) were identified in MSS. The previously reported $N$-nitroso- $n$-butylmethylamine was found in the part of the collection system where artifactual formation was possible. The artifactual formation of NNAs during smoke generation, separation, and analysis 
has been a recognized problem since the first NNA identification in MSS (196-198).

Besides identifying several volatile NNAs in burley tobacco smoke with a procedure that precluded artifactual formation, FREDRICKSON (199) demonstrated that MSS volatile NNA levels are reduced $(60-85 \%)$ by a plasticized cellulose acetate filter, a finding subsequently confirmed by others (200-202). This reduction of volatile NNA levels by selective filtration resembles that observed for phenols (203,204).

Concern over phenols and their promotion effect diminished after reports of removal of significant amounts of them from MSS by selective filtration. While concern about volatile NNAs did diminish, a new NNA concern arose: one involving tobacco-specific $N$-nitrosamines (TSNAs), a class of NNAs newly identified in tobacco and tobacco smoke, namely NNN and NAB.

Artifactual formation of volatile NNAs during smoke collection and analysis was noted in the mid-1960s by NEURATH et al. (194) and FREDRICKSON (199). The problem was once again revisited by KRULL et al. (196) who proposed methodology to reduce it. The problem resurfaced several times in the next decade in the determination of both the volatile NNAs $(197,198)$ and TSNAs in tobacco smoke (198) and preventative measures were proposed.

HOFFMANN and HECHT (7) did not acknowledge that the MSS levels listed for both volatile NNAs and TSNAs may be incorrect (and high) because of their artifactual formation during MSS (and SSS) collection for analysis (198). The US EPA (8) accepted without question the per cigarette MSS volatile NNA and TSNA data listed by HOFFMANN and HECHT (7), and these data were also cited by the SURGEON GENERAL (43).

\section{TSNAs INMSS: DIRECT TRANSFER FROMTOBACCO AND CONFLICTING DATA ON FORMATION DURING THE SMOKING PROCESS}

Nicotine, nornicotine, anabasine, and anatabine are precursors of TSNAs in tobacco and tobacco smoke $(205,206)$. Both nicotine and nornicotine are considered to be NNN precursors. Since NNAs (both volatile and tobacco-specific) occur in tobacco, a part of the NNAs in cigarette MSS was reported to be due to direct transfer of NNAs from tobacco to MSS, the remainder due to formation and transport during the smoking process (206). For NNK, the transfer from tobacco to MSS ranges from $6.9 \%$ to $11.0 \%$ of the amount in the tobacco; this represents about $30 \%$ of the NNK in MSS. Similarly, about $40 \%$ of the NNN in MSS is transferred from the tobacco. According to HOFFMANN and his colleagues, the remainder of these two TSNAs in MSS is formed during the smoking process $(207,208)$. Like the levels of the volatile NNAs in MSS, the levels of the TSNAs in MSS are proportional to the nitrate content of the tobacco filler (209). However, the premise of the pyrogenesis of NNN and NNK has been challenged by FISCHER et al. $(210,211)$ who reported that these compounds occur in cigarette MSS only by transfer from the tobacco rod. CASTONGUAY, a frequent co-author with HOFFMANN and HECHT on TSNA articles, commented that NNK is transferred from tobacco to smoke during the cigarette smoking process (212). In agreement with FISCHER et al., RENAUD et al. (213) concluded from their data on MSS TSNA levels that direct tobacco-to-smoke transfer is the dominant factor explaining the presence of TSNAs in MSS. In a study of the contribution of ${ }^{13} \mathrm{C}$-nicotine to the ${ }^{13} \mathrm{C}-\mathrm{NNN}$ and ${ }^{13} \mathrm{C}$-NNK levels in cigarette MSS condensate, MOLDOVEANU et al. concluded that NNN and NNK are generated during the smoking process (214), thus contradicting the views of FISCHER et al., RENAUD et al., and CASTONGUAY. Moreover, the pyrogenesis situation is further clouded by data on the effect of tobacco nitrate on the TSNA levels in MSS (215). Analysis of MSS TSNAs indicates that NNN and NAT levels increase when nitrate is added to the tobacco but the NNK level does not.

\section{RISK ASSESSMENTS OF TSNAs IN CIGARETTE MSS}

Several investigators have assessed the risk to the smoker of long-time exposure to NNAs in cigarette MSS, particularly the TSNAs NNK and NNN. HOFFMANN and HECHT (7) discussed the effect on a cigarette smoker of inhaling the MSS from a 1986 American nonfiltered cigarette that delivered $425 \mathrm{ng}$ of NNK in its MSS. This delivery can be assessed in an alternate way as recently outlined by TRICKER (32). One can calculate not only the number of packs of cigarettes which would have to be smoked per day for 40 years but also the number of years of smoking 2 packs/day to achieve the same total TSNA exposure as the lowest dose required to induce a significant incidence of lung tumors in laboratory animals.

HECHT and HOFFMANN concluded that the PAHs and NNK are the major carcinogens involved in lung cancer induction by cigarette MSS (216). The inclusion of the PAHs was remarkable in light of numerous publications from the 1960 s to 1993 in which it was reported that $\mathrm{B} a \mathrm{P}$ alone, all the tumorigenic PAHs acting additively, and the tumorigenic PAHs plus promoting phenols account for only a small percentage $(<5 \%)$ of the $\%$ TBA observed.

In Table 12, the TRICKER calculations are applied to NNK and NNN data for the MSS from the 1R4F cigarette, data from RJRT and RICKERT and WRIGHT (217). The calculations differ slightly from those by TRICKER, being applied to smokers of 1 pack/day of the 1R4F cigarette for 35 years. A major problem with these risk assessments is the total disregard of the admonitions made in 1941 by SHEAR and LEITER (99). They wrote:

[T] he term "carcinogenic potency" as used in [carcinogenesis] studies is not to be considered as an invariable property inherent in a compound but is merely a summary of the results of particular experiments and is valid only for animals of the species, strain, sex, age, diet, etc., of the particular animal employed, as well as for the dose, menstruum, mode and site of application, etc., of the compound in question ...

Conclusions regarding the potency of any given compounds should therefore be interpreted in the light of the data upon which they are based.

These admonitions were considered sufficiently meaningful that HARTWELL cited them in the Introduction to his USPHS compendium on compounds tested for tumorigenicity (101). 
Table 12. Extrapolation of rodent bioassay results to a human smoker of cigarette $1 \mathrm{R} 4 \mathrm{~F}$

\begin{tabular}{|c|c|c|c|c|c|c|}
\hline \multirow[b]{2}{*}{ TSNA } & \multirow[b]{2}{*}{$\begin{array}{l}\text { Laboratory } \\
\text { animal }\end{array}$} & \multirow[b]{2}{*}{$\begin{array}{c}\text { Lowest total dose } \\
\text { (mg/kg body weight) }\end{array}$} & \multicolumn{2}{|c|}{ Comparison to man ${ }^{b}$} & \multicolumn{2}{|c|}{ Comparison to $\operatorname{man}^{\mathrm{c}}$} \\
\hline & & & $\begin{array}{l}\text { Packs/day } \\
\text { for } 35 \text { years }\end{array}$ & $\begin{array}{c}\text { Years of smoking } \\
1 \text { pack/day }\end{array}$ & $\begin{array}{l}\text { Packs/day } \\
\text { for } 35 \text { years }\end{array}$ & $\begin{array}{c}\text { Years of smoking } \\
1 \text { pack/day }\end{array}$ \\
\hline \multirow[t]{6}{*}{ NNK } & F344 rat & 70.5 (buccal) (218) & 199 & 6980 & 221 & 7750 \\
\hline & F344 rat & 35.2 (р.о.) (219) & 99 & 3480 & 110 & 3870 \\
\hline & F344 rat & 6.0 (s.c.) (220) & 17 & 594 & 19 & 659 \\
\hline & $\mathrm{A} / \mathrm{J}$ mouse & 364 (р.о.) (221) & 1028 & 36000 & 1141 & 40000 \\
\hline & $\mathrm{A} / \mathrm{J}$ mouse & 20.8 (i.p.) (222) & 59 & 2060 & 65 & 2290 \\
\hline & SG hamster & 9.0 (s.c.) (223) & 25 & 891 & 28 & 989 \\
\hline \multirow[t]{2}{*}{ NNN } & F344 rat & 531 (s.c.) (224) & 1264 & 44200 & 1362 & 47800 \\
\hline & $\mathrm{A} / \mathrm{J}$ mouse & 2153 (i.p.) (225) & 5126 & 179000 & 5520 & 194000 \\
\hline
\end{tabular}

\footnotetext{
${ }^{a}$ Lowest total dose required to induce a significant incidence of lung tumors.

${ }^{b}$ Hypothetical total human experience of a 1 pack/day smoker for 35 years $=0.354 \mathrm{mg}$ NNK and $0.42 \mathrm{mg}$ NNN. These are derived from the RJRT per cigarette data for Cigarette 1R4F; NNK (97 ng), NNN (115 ng).

c Hypothetical total human experience of a 1 pack/day smoker for 35 years $=0.319 \mathrm{mg}$ NNK and $0.39 \mathrm{mg}$ NNN. These are derived from the Rickert and Wright per cigarette data (217) for Cigarette 1R4F; NNK (87 ng), NNN (107 ng).
}

In over 60 years, nothing has been discovered that renders these words invalid! Thus, it is inappropriate to extrapolate findings from a fed or injected or skin-painted compound administered individually to laboratory animals either neat or in solution to the effect of that compound as a component of an extremely complex mixture such as the cigarette MSS aerosol encountered by inhalation. This sentiment was expressed over two decades ago by GORI (226):

[I]t would be unrealistic to assess the biologic effect of any smoke component or additive as an independent entity, outside of the interactions that occur in smoke.

Another problem with the induction of lung tumors in laboratory animals is the omission of the fact that most lung tumors developed by mice are adenomas. Known since the 1950 s is the fact that certain mouse strains are inbred to be susceptible to adenoma development, e.g., $90 \%$ of untreated Strain A mice develop and die from adenomas (227). Administration of a tumorigen does not usually alter the $\%$ adenoma-bearing animals but may shorten the time of adenoma appearance.

\section{TECHNOLOGIES TO CONTROL MSS TOXICANT LEVELS}

Previously (Section 19), we outlined the three criteria used to define a "safer" or "less hazardous" cigarette, i.e., 1) the per cigarette delivery of a specific toxicant has been lowered, 2) the ratio of the specific toxicant to MSS "tar" has been lowered, and 3) the specific tumorigenicity of the MSS "tar" as measured in the mouse skin-painting bioassay has been lowered.

Significantly, the elimination of the first criterion as a complete definition per se of a "safer" or "less hazardous" cigarette and the requirement that all three criteria in the definition be met arose because personnel at various research institutions wished to avoid the appearance of endorsing low-"tar" cigarettes.

Since the early 1950s, it might appear that the cigarette design efforts of the Tobacco Industry R\&D personnel were primarily directed to meeting these criteria. However, the R\&D personnel in general were troubled by the overall definition and viewed two of the criteria as seriously flawed. Criticisms of these criteria were not limited to Tobacco Industry scientists but were also expressed by scientists with anti-tobacco smoking views.

Various members of the anti-tobacco smoking group expressed conflicting opinions on the first criterion. Some interpreted the experimental evidence of lower \% tumorbearing animals in mice treated with reduced levels of "tar" (equivalent to reduced cigarette delivery) as an indication that a lower-"tar" delivery cigarette is "safer" or "less hazardous" than a higher-"tar" delivery cigarette. Others held the view that the biological response resulted from a dose-response factor.

The second criterion for a "safer" or "less hazardous" cigarette is paradoxical. On the one hand, some of its proponents recommended the reduction of the levels of specific components in MSS supposedly responsible for the observed tumorigenicity of particulate matter to mouse skin. However, on the other hand, other proponents of this criterion admitted either an inability to explain the observed biological effect on the basis of the levels of these components in the particulate matter or they accepted (and still accept) the lack of an association between the observed biological effect and chemical composition!

The third criterion suffers from several problems: It ignores the findings that a) inhalation studies with laboratory animals exposed to cigarette MSS have consistently given inconclusive (negative) results with regard to carcinoma induction, b) mouse skin-painting bioassays with cigarette smoke particulate matter do not measure smoke components reported to be tumorigens in other systems, e.g., NNAs, and c) skin-painting and Ames test data with cigarette MSSs produced under certain conditions are widely divergent. Recently, some departure from the third criterion has occurred with the increased usage of various cytotoxicity tests. 


\section{CIGARETTE DESIGN TECHNOLOGIES STUDIED AND REJECTED}

By the early 1960s it was obvious that attempts to reduce the levels of individual MSS toxicants or classes of toxicants, while successful per se, led to unanticipated problems. For example, organic solvent extraction of tobacco removed lipophilic components known or suspected to be precursors of MSS PAHs, the delivery levels of the MSS PAHs were reduced, but the specific tumorigenicity of the CSC from extracted tobacco cigarettes was not reduced proportionately.

Solvent extraction of tobacco, while removing lipophilic $\mathrm{PAH}$ precursors, increases the levels of nitrate and the biopolymers lignin, cellulose, pectins, and starch in the extracted tobacco by a factor of $8 \%$ to $12 \%$. Increasing lignin and carbohydrates levels, known phenols precursors, increases delivery of MSS phenols classified as promoting toxicants. However, assertions that phenols are promoters were offset by reports that a) almost complete removal of phenols from cigarette MSS by selective filtration produces little change in the specific tumorigenicity of the CSC to mouse skin (110,204,228-230) and b) phenol, supposedly the most potent promoter of PAH tumorigenicity, inhibits $\mathrm{B} a \mathrm{P}$ tumorigenicity to mouse skin (152). Increasing the tobacco carbohydrates level also increases the MSS levels of several aldehydes, ketones, and acids defined as in vitro ciliastats. Here again, their importance as contributors to respiratory tract cancer induction was substantially diminished when studies in smokers revealed that a large proportion of most in vitro ciliastats never reach the ciliated areas of the lung (183).

While the presence of NNAs in MSS had been predicted in 1962 (231), their presence in cigarette MSS and the positive relationship between tobacco nitrate level and the NNA levels in tobacco and smoke were not defined until later. Thus, organic solvent extraction of tobacco might be categorized as beneficial because of reduction of mouse-skin tumorigen levels (PAHs) in the MSS but categorized as detrimental because of the increase in MSS levels of other toxicants, the supposed promoters (phenols), cocarcinogens (phenols), ciliastats (vapor-phase aldehydes, ketones, acids), and organ-specific tumorigens (NNAs).

Unknown in the 1950s was the fact that the extraction also removed tobacco components subsequently reported to be inhibitors (alkanes) of $\mathrm{B} a \mathrm{P}$ tumorigenicity $(126,203)$ or anticarcinogenic ( $\alpha$-tocopherol, duvanediols) against the potent tumorigens $\mathrm{B} a \mathrm{P}$ and $\mathrm{DBA}(9)$. Absence or significant depletion of these inhibitors and anticarcinogens from the extracted tobacco was accompanied by substantial reduction of their delivery levels in cigarette MSS. As a result of these and other factors, the process of solvent extraction of tobacco as a cigarette design technology was abandoned. Investigators outside of the Tobacco Industry classified the process as "impractical both technically and economically" (232) and "of academic interest only" (233), a sentiment echoed by the US SURGEON GENERAL [see p. 114, Table 26 in (43)].

The second method studied to reduce PAH levels in cigarette MSS was the use of "catalysts" to modify the combustion process during smoking. The most effective were nitrates that during smoking generated NO that interfered with the free radical mechanism involved in PAH formation. For several years prior to identification of NNAs in tobacco and tobacco smoke, increasing the blend nitrate level was examined as a means to lower the tumorigenic PAH levels in MSS and the specific tumorigenicity of the MS CSC to mouse skin. Nitrate addition lowered several classes of MSS toxicants, the PAHs $(57,110,123,230,234,235)$ and phenols $(230,236)$. Because tobacco stems were usually high in nitrate, inclusion of stembased reconstituted tobacco sheet (RTS) in the blend was proposed and studied (35,237-239). Another way to increase the nitrate level of the blend was to incorporate high-nitrate tobaccos, a technology examined extensively (200,240-242). Because of the demonstration of the relationship between tobacco nitrate level and the NNA levels in MSS $(209,242,243)$, the original proposals were superseded by new ones: Incorporate low-nitrate tobaccos in the blend and/or remove the nitrates from the tobacco (237).

A third method proposed to reduce the level of PAHs in cigarette MSS was the inclusion of a compound in the filter tip that would complex with the PAHs and their nitrogen analogs (244). The aerosol nature of cigarette MSS precludes the success of this approach.

As mentioned previously, research to reduce the levels of individual MSS components or classes of MSS components was replaced by research to reduce MSS components, both vapor-and particulate-phase components, uniformly across the board as much as possible. Such an approach had been voiced by numerous authorities both within and outside of the Tobacco Industry, e.g., DALHAMN's quote of RYLANDER's 1967 comment (245).

Table 13 summarizes the effect of these technologies, eventually rejected, on cigarette MSS properties.

\section{CIGARETTE DESIGN TECHNOLOGIES STUDIED AND INCORPORATED INTO COMMERCIAL PRODUCTS}

In the design of a "less hazardous" cigarette, many approaches have been investigated. Table 14 summarizes the technologies studied by Tobacco Industry and nonIndustry investigators, a list eventually reduced to the eight technologies in Table 15.

Their chronological impact on sales-weighted cigarette MSS "tar" and nicotine deliveries has been noted frequently [cf. Figure 3 in RodGMAn (34)]. By the early 1960s, several cigarette design technologies developed by the Tobacco Industry and used in commercial products were categorized as significant in their contribution to the "less hazardous" cigarette. Ultimately, the initial four design technologies (tobacco blend, effective and efficient filtration, RTS, air dilution via cigarette paper porosity) were increased to eight.

Their significance was recognized in "less hazardous" cigarette design by the $\mathrm{NCI}^{1}$ and the US Surgeon General.

\footnotetext{
${ }^{1}$ All eight cigarette design technologies eventually classified as significant by NCI, US Surgeon Generals, and other investigators on the basis of the 10-year NCI Smoking and Health Program on the "less hazardous" cigarette had been incorporated into one or more US commercial cigarette products prior to the first meeting of the Tobacco Working Group formed in 1968 for the NCI program. In other words, from 1968 to 1978 , no new design technology was generated in the NCI Smoking and Health Program on the "less hazardous" cigarette!
} 
Table 13. Effect of discarded technologies on properties of cigarette mainstream smoke

\begin{tabular}{|c|c|c|c|c|}
\hline \multirow{2}{*}{$\begin{array}{l}\text { Smoke component } \\
\text { and/or property }\end{array}$} & \multirow{2}{*}{$\begin{array}{c}\text { Phase } \\
\text { particulate (PP) } \\
\text { or vapor (VP) } \\
\end{array}$} & \multicolumn{3}{|c|}{ Technology } \\
\hline & & Solvent extraction & Combustion catalyst $^{a}$ & Filter-tip additive ${ }^{b}$ \\
\hline FTC "tar" & PP & $\Downarrow^{c}$ & $\Downarrow$ & $\Downarrow^{d}$ \\
\hline Specific tumorigenicity & PP & $\Downarrow \mathrm{e}$ & $\Downarrow$ & - \\
\hline Specific mutagenicity & PP & $\Uparrow$ & $\Uparrow$ & - \\
\hline $\mathrm{PAHs}$ & PP & $\Downarrow^{c}$ & $\Downarrow$ & $\Downarrow^{d}$ \\
\hline Phenols & PP \& VP & $\Uparrow$ & $\Downarrow$ & - \\
\hline$N$-Nitrosamines & PP \& VP & $\Uparrow$ & $\Uparrow$ & - \\
\hline \multicolumn{5}{|l|}{ Inhibitors/anticarcinogens } \\
\hline Long-chain alkanes & PP & $\Downarrow$ & - & $\Downarrow$ \\
\hline $\mathrm{PAHs}^{\dagger}$ & PP & $\Downarrow$ & - & $\Downarrow$ \\
\hline Duvanediols & PP & $\Downarrow$ & $\Downarrow$ & $\Downarrow$ \\
\hline$\alpha$-Tocopherol & PP & $\Downarrow$ & $\Downarrow$ & $\Downarrow$ \\
\hline
\end{tabular}

\footnotetext{
${ }^{a}$ Nitrates were shown to be the most effective combustion catalysts.

${ }^{\mathrm{b}}$ An additive that forms stable complexes with PAHs and aza-arenes, e.g., chloranil, 2,4,7-trinitrofluorenone.

${ }^{c} \Downarrow$ indicates property or component may be lowered by appropriate choice of blend. $\Uparrow$ indicates property or component may be increased by use of a particular technology.

' Per cigarette deliveries of FTC "tar", PAHs (both tumorigenic and anticarcinogenic) reduced by same percentage due to increase in pressure drop across the additive-treated filter tip.

${ }^{e}$ Decrease in \% TBA was much less than \% decrease in MSS levels of tumorigenic PAHs such as BaP.

${ }^{\dagger}$ Includes naphthalene, anthracene, phenanthrene, fluoranthene, pyrene, benzo[e]pyrene, benzo[b]triphenylene, benz[a]anthracene.
}

Table 14. Alteration of cigarette mainstream smoke yield, composition, and biological activity: Methods studied

\begin{tabular}{lc}
\hline \multicolumn{2}{c}{ Cigarette design technology } \\
\hline $\begin{array}{l}\text { Tobacco selection } \\
\text { Type }\end{array}$ & $\begin{array}{c}\text { Tobacco additives } \\
\text { Combustion modifiers }\end{array}$ \\
$\begin{array}{l}\text { Stalk position } \\
\text { Nitrate content }\end{array}$ & Casing materials and humectants \\
Nicotine content & Flavorants \\
Other components & Pesticides, agricultural chemicals \\
Tobacco treatment & Cigarette paper \\
Curing & Porosity (air dilution) \\
Grading & Additives \\
Fermentation & Coatings \\
Extraction & \\
Denicotinization & Filtration \\
Ammoniation & Efficiency/selectivity \\
Expansion (laminae & Additives \\
and/or stems) & Material (cellulose acetate, paper) \\
& Material (charcoal) \\
Blending & \\
Tobacco cut width & Air dilution (perforated filter tips) \\
Amount of tobacco & Diluents (substitutes) \\
Cigarette dimensions & Cytrel® \\
Tobacco weight & NSM® (New Smoking Material) \\
RTS (nonpaper) & Expanded grains \\
RTS (paper) & Carbon/carbonized filler \\
Homogenized leaf & SSM® (Sutton Smoking Material) \\
Stem inclusion & Other plants (lettuce, peanut hulls, \\
Expanded laminae & etc.) \\
Moisture content & \\
\hline & \\
\hline
\end{tabular}

In Table 15 are listed chronologically (1960 through 1997) some of the reports in which various authorities commended these eight design technologies.

Table 16 summarizes the effect of these technologies plus tobacco ammoniation on some of the major cigarette MSS properties. A technology that primarily influences the particulate-phase yield generally influences the MSS levels of those components defined as particulate-phase toxicants. Similarly, a technology that primarily influences the vaporphase yield generally influences the MSS levels of those components defined as vapor-phase toxicants. From 1913 to the early 1950s, the major design technology employed was the tobacco blend. Chronologically, the rodent skin-painting bioassay became available, specific PAHs were defined as tumorigenic to laboratory animals, but prior to 1954 little was known about the composition of tobacco smoke.

While each design technology may be used to control MSS yield and composition, none is now used individually. The eight design technologies listed in Table 15 are used in concert and to different degrees, thus enabling the design of consumer acceptable cigarettes with MSS FTC "tar" deliveries ranging from 1 to $40 \mathrm{mg} / \mathrm{cig}$ and MSS nicotine deliveries ranging from 0.1 to $3.0 \mathrm{mg} / \mathrm{cig}$.

Of course, the initial thrust of this across-the-board reduction was aimed at reducing the MSS "tar" yield because of extrapolation by WYNDER et al. (246) of their 1957 mouse-skin bioassay findings:

Although it is difficult to estimate a comparable exposure level for man, the human data in line with the animal data indicate that a reduction in total tar exposure will be followed by a decrease in tumor formation. For this reason, measures directed toward this reduction are of utmost importance ... The minimum dose of tar capable of producing papillomas in mice is about one third, and of producing cancer one half, that of the optimum dose ... The practical implications of these data and their relationship to the human cancer problem have been emphasized.

In his 1957 testimony during the filter-tipped cigarette hearings, WYNDER (36) reiterated his opinion that reducing "tar" exposure dose by $40 \%$ to $50 \%$ would substantially reduce lung cancer induction in smokers.

Examination of the graphical representation of the salesweighted average "tar" yield for US commercial cigarettes 
Table 15. Cigarette design technologies recognized as contributing to less hazardous cigarettes ${ }^{a, b}$

\begin{tabular}{|c|c|c|c|c|c|c|c|c|c|c|c|c|c|c|c|}
\hline Design technology & $\begin{array}{l}\text { W\&H } \\
1960 \\
\end{array}$ & $\begin{array}{l}\text { W\&H } \\
1964 \\
\end{array}$ & $\begin{array}{l}\text { W\&H } \\
1965 \\
\end{array}$ & $\begin{array}{l}\text { W\&H } \\
1966 \\
\end{array}$ & \begin{tabular}{|l} 
W\&H \\
1967 \\
\end{tabular} & $\begin{array}{l}\text { W\&H } \\
1969 \\
\end{array}$ & \begin{tabular}{|c} 
W\&He \\
1976
\end{tabular} & $\begin{array}{c}\mathrm{NCl} \\
1976-80\end{array}$ & $\begin{array}{c}\text { HSHW } \\
1978 \\
\end{array}$ & \begin{tabular}{|c} 
US SG \\
1979 \\
\end{tabular} & $\begin{array}{l}\text { W\&H } \\
1979 \\
\end{array}$ & $\begin{array}{c}\text { LHHW } \\
1980\end{array}$ & \begin{tabular}{|c} 
US SG \\
1981
\end{tabular} & $\begin{array}{l}\text { H\&W } \\
1986 \\
\end{array}$ & $\begin{array}{l}\mathrm{H} \& \mathrm{H} \\
1997 \\
\end{array}$ \\
\hline Tobacco blend ${ }^{\mathrm{c}}$ & $\mathrm{x}$ & $\mathrm{x}$ & $\mathrm{x}$ & $\mathrm{X}$ & $\mathrm{x}$ & $\mathrm{x}$ & $\mathrm{X}$ & $\mathrm{X}$ & $\mathrm{x}$ & $\mathrm{X}$ & $\mathrm{x}$ & $\mathrm{X}$ & $\mathrm{x}$ & $\mathrm{x}$ & $\mathrm{x}$ \\
\hline Filter tip ${ }^{d}$ & $x$ & $x$ & $x$ & $x$ & $x$ & $x$ & $x$ & $x$ & $x$ & $x$ & $x$ & $x$ & $x$ & $x$ & $x$ \\
\hline Filter-tip additive ${ }^{e}$ & - & $x$ & $x$ & - & $x$ & $x$ & $x$ & $\mathrm{x}$ & $x$ & $\mathrm{x}$ & $x$ & $x$ & $x$ & $x$ & $\mathrm{x}$ \\
\hline RTS $^{f}$ & $\mathrm{x}$ & $x$ & $\mathrm{x}$ & $\mathrm{x}$ & $\mathrm{x}$ & $\mathrm{x}$ & $\mathrm{x}$ & $x$ & $\mathrm{x}$ & $\mathrm{x}$ & $x$ & $\mathrm{x}$ & $\mathrm{x}$ & $\mathrm{x}$ & $x$ \\
\hline Paper additive ${ }^{g}$ & 一 & $x$ & $x$ & - & - & - & - & $x$ & 一 & $x$ & 一 & - & 一 & - & $x$ \\
\hline $\begin{array}{l}\text { Air dilution (paper } \\
\text { porosity) }\end{array}$ & $x$ & $x$ & $x$ & $x$ & $x$ & $x$ & $x$ & $x$ & $x$ & $x$ & $x$ & $x$ & $x$ & $x$ & $x$ \\
\hline Expanded tobacco ${ }^{i}$ & - & - & - & - & - & - & $\mathrm{x}$ & $\mathrm{x}$ & $\mathrm{x}$ & $\mathrm{x}$ & $\mathrm{x}$ & $\mathrm{x}$ & $\mathrm{x}$ & $\mathrm{x}$ & $\mathrm{x}$ \\
\hline $\begin{array}{l}\text { Air dilution (filter-tip } \\
\text { perforation) }\end{array}$ & - & - & - & 一 & 一 & 一 & $x$ & $x$ & $x$ & $x$ & $x$ & $x$ & $x$ & $x$ & $\mathrm{x}$ \\
\hline
\end{tabular}

a Technologies cited in US Surgeon General's 1979, 1981, and 1982 smoking-and-health reports [see pp. 104-114 in (43), see pp. $217-218$ in (172), (253)].

${ }^{\mathrm{b}}$ W\&H 1960 = Wynder and Hoffmann (247); W\&H 1964 = Wynder and Hoffmann (123); W\&H 1965 = Wynder and Hoffmann (248); W\&H 1966 = Wynder and Hoffmann (249); W\&H $1967=$ Wynder and Hoffmann [see p. 503 in (57)]; W\&H $1969=$ Wynder and Hoffmann (250); W\&H 1979 = Wynder and Hoffmann (251); W\&He 1976 = Wynder and Hecht (233); NCl 1976-80 = Gori (35); NCl (35); HSHW 1978 = Hoffmann et al. (252); US SG $1979=$ USPHS [see pp. 104-114 (43)]; US SG 1981 = USPHS (253); LHHW $1980=$ Lavoie et al. (79); H\&W 1986 = Hoffmann and Wynder (254); H\&H 1997 = Hoffmann and Hoffmann (255)

${ }^{C}$ First cigarette containing a blend of flue-cured, burley, and Oriental tobaccos introduced by RJRT (the 70-mm Came/). Maryland tobacco added to blend in 1917. Most cigarettes prior to 1913 were fabricated from a 100\% flue-cured blend or a $100 \%$ Oriental tobacco blend. Post-WWI, the Camel-type blend, the so-called American blend, was copied in most countries; exceptions included UK, Canada.

${ }^{d}$ RJRT introduced the first highly successful filter-tip cigarettes, the Winston, in 1953.

${ }^{e}$ Cellulose acetate filter tip included triacetin as plasticizer. MSS yield and composition subsequently controlled by increase in triacetin level.

${ }^{\mathrm{f}}$ Winston was first marketed cigarette with RTS (no added fiber or adhesive) in the blend. By 1958, all US companies were using RTS. RTS had been used previously as cigar wrapper but not in a cigarette blend.

${ }^{9}$ In 1958, citrates were added to cigarette paper for more uniform combustion of the tobacco rod.

${ }^{\mathrm{h}}$ In 1959, increased cigarette paper porosity was introduced as a means to lower MSS "tar" and nicotine yield.

Expanded tobacco laminae were incorporated into commercial products in the late 1960s. US patents were issued in 1970 (256).

${ }^{j}$ A product with a perforated filter tip was introduced commercially in the US in the late 1960s.

[cf. Figure 3 in RoDGMAN (34)] reveals that the $40 \%$ to $50 \%$ reduction in MSS "tar" yield considered vital by WYNDER in 1957 was achieved in the late 1960s, i.e., a reduction from $38-39 \mathrm{mg} / \mathrm{cig}$ to $19-20 \mathrm{mg} / \mathrm{cig}$. Further examination reveals that by the early 1980s, the sales-weighted average "tar" was further reduced to about $12 \mathrm{mg} / \mathrm{cig}$, i.e., an additional $40 \%$ reduction had been achieved. Corresponding reductions in the MSS deliveries of total PAHs in general, $\mathrm{B} a \mathrm{P}$ in particular [see pp. 111-112 in (43), (257)], and nicotine were also achieved.

Reminiscent of the numerous lengthy review articles issued in the 1980s and early 1990s on the biological properties of NNAs, particularly TSNAs, in MSS $(258,259)$ is the recent flood of highly repetitious articles devoted to discussions of the "changing cigarette" $(12,255,260)$. Actually, the recent articles were preceded by earlier ones, e.g., the 1981 SURGEON GENERAL's report (253), a 1986 HOFFMANN and WYNDER article (254), and a 1990 article by HOFFMANN and HECHT (7).

Several technologies incorporated into commercial products were eventually abandoned because of poor consumer acceptance. One of these was the drastic reduction of tobacco nicotine that resulted in a low, almost zero, MSS nicotine yield. A second was the incorporation of a tobacco substitute that effectively is a diluent for the tobacco. Examples of these include the New Smoking Material ${ }^{\circledR}$ (NSM) from Imperial Tobacco, Cytrel® from Celanese, and the Sutton Smoking Material@ (SSM). Each had its own peculiar problem.

Consumers did not accept commercial products containing NSM ${ }^{\circledR}$ or Cytrel ${ }^{\circledR}$ so they were eventually removed from the marketplace. In the NCI Smoking and Health Program on the "less hazardous" cigarette both NSM ${ }^{\circledR}$ and Cytrel ${ }^{\circledR}$ were examined. The biology of NSM $®$ matched the claims made by the manufacturers whereas they did not for Cytrel®. The MSS from Cytrel ${ }^{\circledR}$ cigarettes was found to contain several dozen components not present in tobacco smoke (GREEN et al., 261). The data from bioassays conducted on Cytrel ${ }^{\circledR}$ MSS in the NCI program (35) fell far short of those presented by Celanese personnel. While the bioassay results in the NCI program on NSM® MSS were satisfactory, the $\mathrm{B} a \mathrm{P}$ :"tar" ratio was three times that of several popular commercial cigarettes.

\section{THE US TOBACCO INDUSTRY CRITICIZED: NO NEW CIGARETTE DESIGN TECHNOLOGY SINCE 1975}

Recently HOFFMANN and HOFFMANN (12) wrote:

Major modifications in the makeup of the commercial cigarette were introduced between 1950 and 1975. Since then, there have been no substantive changes toward a further reduction of the toxic and carcinogenic potential of cigarette smoke beyond reducing MS yields of tar, nicotine, and carbon monoxide. Some of these modifications have also resulted in diminished yields of several toxic and carcinogenic smoke constituents.

As mentioned earlier, examination of the graphical representation of the sales-weighed "tar" and nicotine values for US commercial cigarettes reveals that from 1975 to date the FTC "tar" value has decreased from 18 to $11 \mathrm{mg} / \mathrm{cig}$. The HOFFMANNS obviously overlooked the fact that the eight 
Table 16. Effect of the eight significant technologies plus ammoniation on cigarette mainstream smoke properties ${ }^{a}$

\begin{tabular}{|c|c|c|c|c|c|c|c|c|c|c|c|}
\hline \multirow[b]{2}{*}{$\begin{array}{l}\text { Smoke component } \\
\text { and/or property }\end{array}$} & \multirow{2}{*}{\begin{tabular}{|} 
Particulate \\
(PP) or vapor \\
(VP) phase
\end{tabular}} & \multirow[b]{2}{*}{$\begin{array}{c}\text { Tobacco } \\
\text { blend }\end{array}$} & \multicolumn{2}{|c|}{ Filter tip } & \multirow[b]{2}{*}{ RTS } & \multirow[b]{2}{*}{$\begin{array}{l}\text { Paper } \\
\text { additive }\end{array}$} & \multirow[b]{2}{*}{$\begin{array}{l}\text { Filter-tip } \\
\text { additive }^{c} \\
\end{array}$} & \multicolumn{2}{|c|}{ Air dilution via } & \multirow[b]{2}{*}{$\begin{array}{c}\text { Expanded } \\
\text { tobacco } \\
\end{array}$} & \multirow[b]{2}{*}{$\begin{array}{c}\text { Ammon- } \\
\text { iation }\end{array}$} \\
\hline & & & $\mathrm{CA}^{\mathrm{b}}$ & Carbon & & & & $\begin{array}{l}\text { Paper } \\
\text { porosity }\end{array}$ & $\begin{array}{c}\text { Perforated } \\
\text { filter-tip }\end{array}$ & & \\
\hline FTC “tar" & PP & $\Downarrow$ & $\Downarrow$ & - & $\Downarrow$ & $\Downarrow$ & - & $\Downarrow$ & $\Downarrow$ & $\Downarrow$ & - \\
\hline Specific tumorigenicity & PP & $\Downarrow$ & - & - & $\Downarrow$ & $\Downarrow$ & - & $\Downarrow$ & $\Downarrow$ & $\Downarrow$ & - \\
\hline Specific mutagenicity & PP & $\Downarrow$ & - & - & - & - & - & - & - & $\Downarrow$ & $\Uparrow$ \\
\hline Ciliastasis & VP & $\Downarrow$ & - & $\Downarrow$ & $\Uparrow$ & - & - & $\Downarrow$ & $\Downarrow$ & $\Downarrow$ & - \\
\hline FTC nicotine & PP & $\Downarrow$ & $\Downarrow$ & - & $\Downarrow$ & $\Downarrow$ & - & $\Downarrow$ & $\Downarrow$ & $\Downarrow$ & $\Downarrow$ \\
\hline $\mathrm{PAHs}$ & PP & $\Downarrow$ & $\Downarrow$ & - & $\Downarrow$ & $\Downarrow$ & - & $\Downarrow$ & $\Downarrow$ & $\Downarrow$ & - \\
\hline Aza-arenes & PP & $\Downarrow$ & $\Downarrow$ & $\Downarrow$ & - & $\Downarrow$ & - & $\Downarrow$ & $\Downarrow$ & $\Downarrow$ & - \\
\hline Aromatic amines & $\mathrm{PP}$ & $\Downarrow$ & $\Downarrow$ & $\Downarrow$ & - & - & - & $\Downarrow$ & $\Downarrow$ & $\Downarrow$ & - \\
\hline $\mathrm{N}$-Heterocyclic amines & PP & $\Downarrow$ & $\Downarrow$ & $\Downarrow$ & - & - & - & $\Downarrow$ & $\Downarrow$ & $\Downarrow$ & - \\
\hline NNAs, volatile & VP & - & - & - & - & - & $\Downarrow$ & $\Downarrow$ & $\Downarrow$ & - & - \\
\hline NNAs, nonvolatile & PP & $\Downarrow$ & - & - & - & - & - & $\Downarrow$ & $\Downarrow$ & - & - \\
\hline TSNAS & PP & $\Downarrow$ & $\Downarrow$ & $\Downarrow$ & - & - & - & $\Downarrow$ & $\Downarrow$ & $\Downarrow$ & - \\
\hline Aldehydes & VP & - & - & $\Downarrow$ & $\Uparrow$ & - & - & $\Downarrow$ & $\Downarrow$ & - & $\Downarrow$ \\
\hline Phenols & VP & - & - & $\Downarrow$ & - & - & $\Downarrow$ & $\Downarrow$ & $\Downarrow$ & - & - \\
\hline Phenols & $\mathrm{PP}$ & $\Downarrow$ & $\Downarrow$ & - & - & - & - & $\Downarrow$ & $\Downarrow$ & - & - \\
\hline Miscellaneous organic & VP \& PP & - & - & - & - & - & - & $\Downarrow$ & $\Downarrow$ & - & - \\
\hline $\mathrm{CO}$ & VP & - & - & - & $\Uparrow$ & - & - & $\Downarrow$ & $\Downarrow$ & $\Downarrow$ & - \\
\hline
\end{tabular}

\footnotetext{
a $\Downarrow$ Indicates property or component may be lowered by appropriate choice of blend, $\Uparrow$ indicates property or component may be increased by use of a particular technology.

${ }^{\text {a }} \mathrm{CA}=$ cellulose acetate.

${ }^{\mathrm{C}}$ The filter-tip additive in this case is a plasticizer such as triacetin or Carbowax®.
}

technologies used in concert and to different degrees have resulted in this change ( 40\%) in the FTC "tar" yield from 1975 to date. The decrease in FTC "tar" of more than $50 \%$ from 1955 though 1975 attained and surpassed the goal originally proposed by WYNDER in 1957 to resolve the lung cancer situation (36).

Recent claims (see Table 1, Footnote e) that a new cigarette product is the first with lowered levels of carcinogens in its smoke are obviously erroneous. Examination of the salesweighted average FTC "tar" and nicotine yield from 1955 to date indicates that the "tar" and nicotine have decreased substantially. Correspondingly, the MSS deliveries of $\mathrm{B} a \mathrm{P}$ and other PAHs have decreased not only on a per cigarette basis but also on a per milligram of "tar" delivered basis. This and the decreased specific tumorigenicity of CSC to mouse skin from that observed in 1955 were acknowledged in 1979 by the US SURGEON GENERAL [see pp. 111-112 in (43)].

\section{TSNAs IN FLUE-CURED TOBACCO: BACK TO THE FUTURE}

In a previous section, we discussed the development and utilization of cigarette design technologies that more or less uniformly and simultaneously reduced the levels of toxicants in MSS particulate and vapor phases. After several decades of such activities, examination of the effect of lowering specific tobacco components on the level of specific toxicants in and the biological properties of MSS has once again been implemented. The targets of choice are the TSNAs and the $N$-heterocyclic amines.

As described by WiLLIAMS (262) there is general agreement among tobacco scientists that TSNAs are not present in either freshly harvested, i.e., green flue-cured and burley tobaccos. As the tobaccos are cured either by air-curing in the case of burley or in heated barns for flue-cured varieties, the amounts of TSNAs rise to their final levels. In the case of air-curing, the process has changed little for the past fifty years. However, for flue-curing, the process changed drastically in the US during the 1960s and 1970s due to the introduction of energy efficient bulk-curing barns heated directly by the exhaust gases of liquid propane gas or similar burners. It is at this point that a breakdown must have occurred between tobacco agriculturists and chemists. The emission of $\mathrm{NO}_{2}$ during the combustion of liquid propane or natural gas is well known. In fact, the North Carolina Department of Environment and Natural Resources (NCDENR) has electronic spreadsheets available for download from its website that North Carolina industries may use in estimating their $\mathrm{NO}_{2}$ emissions during natural gas or liquid propane combustion. In retrospect, any competent chemist would predict the potential nitrosation of tobacco alkaloids during flue-curing in the presence of combustion exhaust gases. However, without the knowledge of TSNA formation during direct-heating of green tobaccos, the agricultural community adopted the new energy-efficient technique. It appears that prior to this "technological advance", the formation of TSNAs during flue-curing by traditional methods was not a problem.

Rather than using the existing knowledge, at least two research groups during recent years have used the Edisonian approach to discover the problem with direct heating flue-curing of tobacco. PEELE et al. (263) demonstrated that modification of the curing process for flue-cured tobacco permitted significant control of its TSNA levels. The curing process was altered from one involving direct-fired burners 


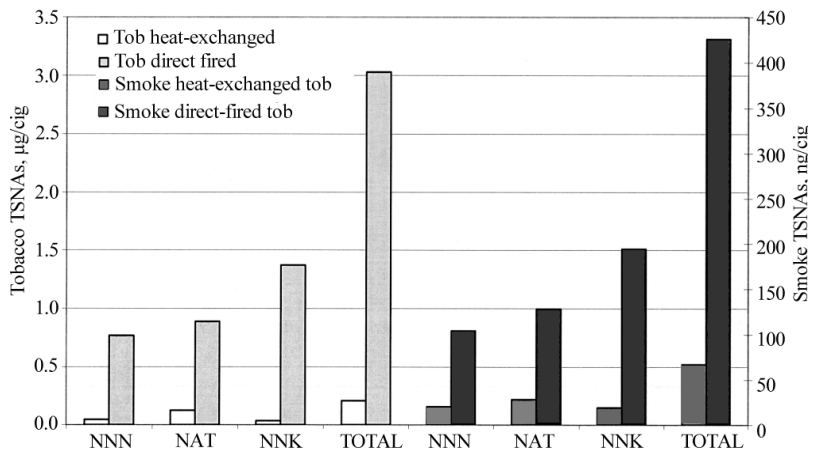

Figure 3. Comparison of TSNAs in tobacco and smoke of heat-exchanged and direct-fired flue-cured tobaccos.

to one involving a heat exchange system. During approximately the same time period, WILLIAMS (262) applied for and was granted a US patent on essentially the same modification of the flue-curing barns to achieve the same significant reduction in TSNAs. An example of the TSNA reductions in flue-cured tobacco and its smoke is shown in Figure 3. The tobacco data are taken from WILLIAMS and the smoke data are taken from DooliTTLE et al. (264). As a result of these two disclosures, one through scientific communication and the other through the patent literature, several legal actions have taken place. In May 2001, Star Scientific, Inc., co-founded by Jonnie R. Williams and Francis E. O’Donnell Jr, initiated patent infringement litigation against R.J. Reynolds Tobacco Company, i.e., the employer of PEELE et al. Meanwhile, on June 28, 2002, PHILIP MORRIS USA (265) petitioned the US legal system to declare the patent of WILLIAMS et al. to be invalid and unenforceable. A recent ruling dismissed the lawsuit of PHILIP MORRIS USA (266).

Regardless of the legal proceedings outcome, two issues arise. On the first issue there appears to be no disagreement from anyone. Discontinuing direct heating for flue curing is desirable from a product stewardship perspective. Every practical effort should be made to reduce the amounts of alleged human carcinogens from tobacco products. However, whether the reduction or elimination of TSNAs from MSS will result in a "less hazardous" cigarette is unknown. Earlier, we have presented pure compound data in Tables 1 and 3 that indicate TSNAs play a minor role in MSS carcinogenesis. Additionally, from a comparison of the biological effect (Neutral Red cytotoxicity, mutagenicity in the Ames test with several Salmonella typhimurium strains) of the MS CSCs from flue-cured tobacco cigarettes with normal and reduced levels of TSNAs, DOOLITTLE et al. (264) reported no significant difference between the biological activity of the two CSCs. Although the DoOLITTLE et al. data appear to support the hypothesis on a whole-smoke basis that MSS TSNAs are of relatively minor toxicological importance, the sensitivity of the Ames assay is not sufficient to differentiate between the cigarettes tested. For example, consider the following points published by DOOLITTLE et al.:

- The minimum amount of NNK needed for a mutagenic response in the Ames assay is $200 \mu \mathrm{g}$.

- The maximum amount of CSC that can be tested is $250 \mu \mathrm{g}$.

- In $250 \mu \mathrm{g}$ of CSC there is 1.33 and $0.13 \mathrm{ng}$ of NNK from direct fired and heat exchanged flue-cured tobacco, respectively.

- The amount of NNK in the CSC from either flue-cured tobacco smoke is too low for a response.

Just as analytical chemists must keep in mind limits of detection, biologists must also be aware of their assay limits.

A major class of MSS components to attain notoriety recently, the $N$-heterocyclic amines - the so-called Sugimura compounds - were initially identified as components of protein pyrolysates and cooked protein-containing foods. Despite their inordinately high mutagenicity in the Ames test (Table 17), their tumorigenicity to laboratory animals (267), and their inclusion in recent lists of MSS toxicants (255), no $N$-heterocyclic amine in MSS has received the attention of such components as $\mathrm{B} a \mathrm{P}$ or NNK. However, ClAPP et al. (268) reported that removal of protein from flue-cured and burley tobacco produces significant reductions in the mutagenicity (Ames test, Salmonella typhimurium strains TA98 and TA100) of the CSCs from both reduced protein flue-cured and burley tobacco products.

Pyrolysis of glutamic acid and tryptophan yield several $\mathrm{N}$ heterocyclic amines, e.g., Glu-P-1, Glu-P-2, Trp-P-1, and Trp-P-2. These four $N$-heterocyclic amines were subsequently identified not only in cooked foods but also as tobacco smoke components. Their precursors in foods and tobacco smoke are considered to be glutamic acid and tryptophan, either bound in a protein or as the free amino acid.

Table 17. Mutagenic activities (revertants $/ \mu \mathrm{g}$ ) of $\mathbf{N}$-heterocyclic amines towards Salmonella typhimurium ${ }^{\text {a }}$

\begin{tabular}{|c|c|c|c|c|c|}
\hline & & \multicolumn{2}{|c|}{ TA98 } & \multicolumn{2}{|c|}{ TA100 } \\
\hline \multicolumn{2}{|c|}{ Compound (designation) } & Lee et al. (165) & Sugimura (166) & Lee et al. (165) & Sugimura (166) \\
\hline $\mathrm{IQ}$ & $\{2$-amino-3-methylimidazo[4,5-f]quinoline $\}$ & 222000 & 433000 & 11000 & 7000 \\
\hline MelQ & $\{2$-amino-3,4-dimethylimidazo[4,5-f]quinoline $\}$ & 1327000 & 661000 & 70000 & 30000 \\
\hline Glu-P-1 & \{2-amino-6-methyldipyrido[1,2-a:3',2'-d]imidazole $\}$ & 73000 & 49000 & 4000 & 3200 \\
\hline Glu-P-2 & $\{2$-aminodipyrido[1,2-a:3',2'- $d$ ]imidazole $\}$ & 600 & 1900 & 400 & 1200 \\
\hline Trp-P-1 & $\{3$-amino-1,4-dimethyl-5H-pyrido[4,3- $b]$ indole $\}$ & 20000 & 39000 & 500 & 1700 \\
\hline Trp-P-2 & $\{3$-amino-1-methyl-5H-pyrido[4,3-b]indole $\}$ & 一 & - & 2000 & 1800 \\
\hline $\mathrm{BaP}$ & $\{$ benzo[a]pyrene $\}$ & 200 & 一 & - & - \\
\hline
\end{tabular}

\footnotetext{
a Tests with Salmonella typhimurium involved use of S-9 mix.
} 


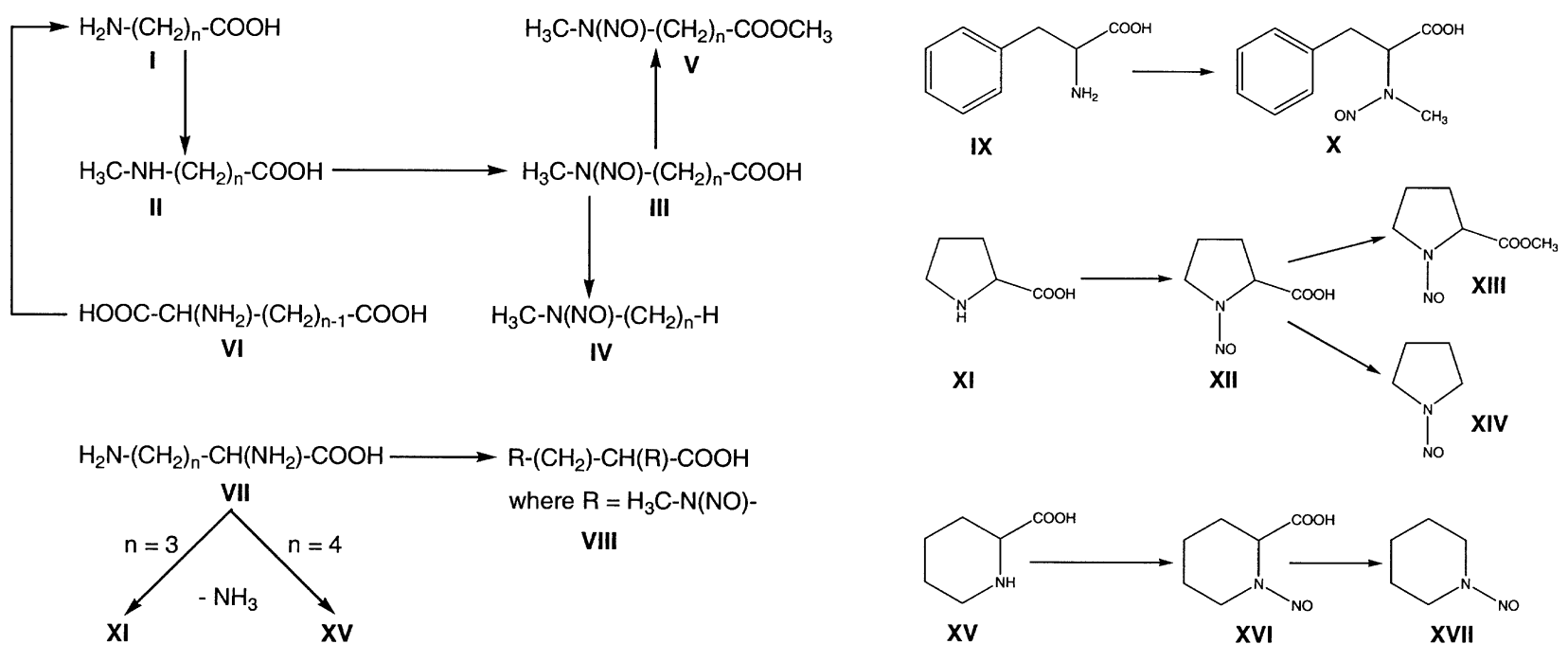

Figure 4. Relationship among amino acids, $\mathrm{N}$-nitrosamino acids, their esters, and $\mathrm{N}$-nitrosamines

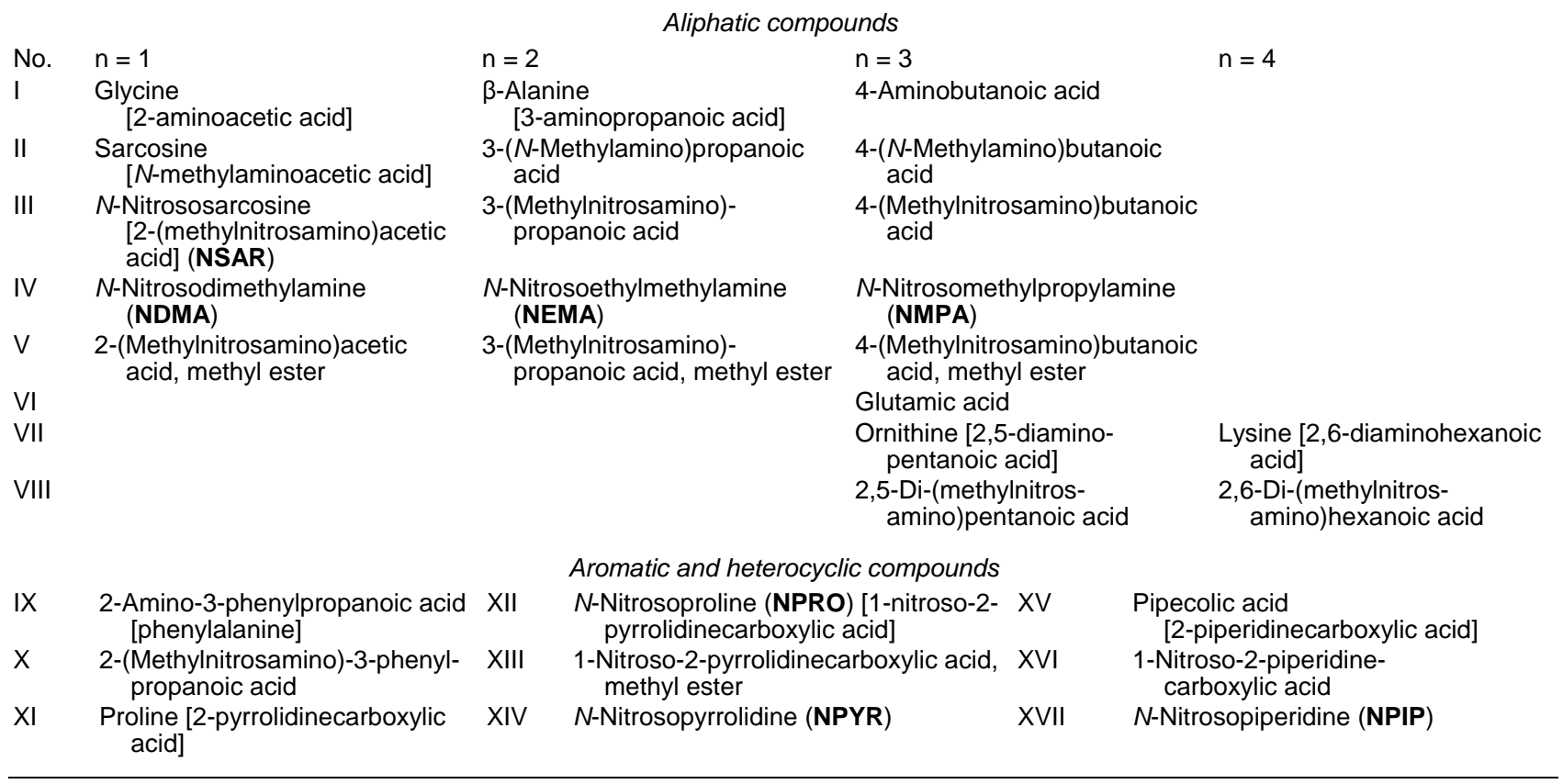

During smoking, the pyrogenesis of amino acids would be reduced from reduced protein tobacco.

The importance of the role played by amino acids in tobacco (and indirectly the tobacco proteins) as precursors of NNAs in tobacco smoke may be seen by examination of the information in Figure 4. Several amino acids identified in tobacco and/or tobacco smoke are listed in Table 18. At least eight are involved directly or indirectly as precursors of NNAs that account for almost half of the NNAs identified in tobacco and tobacco smoke. Reduction of the levels of these amino acids or the tobacco protein source should reduce the levels of NNAs in tobacco and smoke. Thus, removal or reduction of the levels of the proteins and amino acids in tobacco serves two purposes: a) Reduction of the levels in tobacco smoke of the $N$-heterocyclic amines and $b$ ) reduction of the amino acid-derived NNAs in tobacco and smoke.

\section{DISCUSSION}

While we do $\mathrm{n}$ ot dispute the inherent risks of cigarette smoking, throughout our review we have tried to put several issues in perspective. The number of MSS toxicants listed by various individuals, institutions, and government agencies has increased steadily over the past few decades. However, with a few exceptions, it is obvious that the exposure of a pack-a-day smoker to the listed MSS toxicants (Tables 1 and 2) is much less than the exposure in the workplace permitted by or acceptable to OSHA (Table 3).

While we have noted the problem inherent in extrapolation of biological effects observed in laboratory animals treated by various administration methods with exaggerated doses of a specific substance to the effect on the smoker inhaling a cigarette MSS aerosol containing a much smaller dose of 
Table 18. Amino acids in tobacco and/or tobacco smoke

\begin{tabular}{|c|c|}
\hline$\alpha$-Alanine & Leucine \\
\hline$\beta$-Alanine ${ }^{a}$ & Lysine $\{2,6 \text {-diaminohexanoic acid }\}^{a}$ \\
\hline Aspartic acid & Ornithine $\{2,5 \text {-diaminopentanoic acid }\}^{a}$ \\
\hline 2-Aminobutanoic acid & Phenylalanine $^{a}$ \\
\hline 4-Aminobutanoic acid ${ }^{a}$ & Proline $^{a}$ \\
\hline Cysteine & Serine \\
\hline Glutamic acid $^{\text {a,b }}$ & Tryptophan $^{\mathrm{b}}$ \\
\hline Glycine $^{a}$ & Valine \\
\hline
\end{tabular}

a The amino acid is involved in $N$-nitrosamine formation.

b The amino acid is involved in $\mathrm{N}$-heterocyclic amine formation.

that substance admixed with nearly 4800 other identified substances (and, as suggested by WAKEHAM (269), possibly as many as 100000 substances), a detailed discussion of the problem is beyond the scope of this paper. Other highly capable authorities have spoken at length to the problem.

The repeated assertion since the advent of the low-"tar" cigarette that increased levels of added ingredients to cigarette tobacco have increased the levels of MSS toxicants and the adverse MSS biological effect is without merit. No evidence to prove such an assertion has ever been presented, but much data contradicting the assertion have been published $(16-18,81,82,87)$. This assertion is remindful of many others made over the years that are not supported by credible evidence (176).

Although we have dealt at length with the many lists of MSS toxicants, we have also questioned why similar lists (Table 11) are seldom generated for MSS components known to counteract or diminish the adverse biological activity of many of the listed MSS toxicants.

Over the years, various individuals, institutions, and agencies opposed to cigarette smoking have not only acknowledged the significance of the Tobacco Industry's development and use of cigarette design technologies to lower the levels in MSS of the toxicants but also commended the Industry for its activities in this regard. However, even investigators as zealous as Wynder and Hoffmann were aware of one of the major problems, if not the major problem, in the design of a "less hazardous" cigarette, namely, acceptance by the consumer. When the low-"tar" cigarette had obviously become the choice of many smokers, WYNDER and HOFFMANN, after commending the Tobacco Industry for its emphasis on low"tar" cigarette marketing, noted (251):

Development of a less harmful cigarette acceptable to the majority of the smokers needs to continue. We must be realists. A completely safe cigarette smoked by only $1 \%$ of the smoking public is of considerably less societal benefit than a cigarette with some adverse effects smoked by $90 \%$ of the public.

WYNDER reiterated the above statement on another occasion (270):

[I]t is important to appreciate that a virtually harmless cigarette smoked by only $1 \%$ of the population will have a lesser impact on the reduction of tobacco-related diseases than a somewhat more harmful cigarette smoked by $80 \%$ of the total smoking population. Research on the less harmful cigarette should therefore be directed toward developing a cigarette containing the lowest possible amount of harmful elements for all tobacco-related diseases, but one that has sufficient acceptability for the largest segment of smokers.

GORI expressed a similar sentiment in 1977 (271) and in his summary of the 1979 Banbury Conference on the "less hazardous" cigarette (226). However, at that time he also considered that a weaning process - the sequential changing by a smoker to acceptable lower and lower "tar" delivery cigarette brands - would ultimately attain the goal of complete cessation (272).

We have noted that the recent criticism of the Tobacco Industry for its failure to generate any new significant cigarette design technologies since 1975 (12) is totally without merit. The eight design technologies deemed significant (Table 15), when used in concert but to different degrees since 1975, have continued to reduce the salesweighted FTC "tar" substantially below the goal originally recommended, i.e., a 50\% reduction from the mid-1950 "tar" yield (36). None of the critics has ever developed a cigarette design technology to match the significance of the eight in US and worldwide commercial cigarette production since the late 1960s. The attitude of the critics and the contrasting performance by the Industry remind us of a statement by ARISTOTLE (273):

In practical matters, the end is not mere speculative knowledge of what is to be done, but rather the doing of it.

Four centuries ago, long before the development of our present skills in chemical separations and analyses and toxicological techniques, ROWLAND categorized inhaled and exhaled tobacco smokes as "toxicants" in an epigram (274):

But this same poyson, steeped India weede,

In head, hart, lunges, do soote and copwebs breede.

With that he gasp'd, and breath'd out such a smoke

That all the standers by were like to choke.

ROWLAND, with absolutely no knowledge of the composition of tobacco smoke, could not define any specific component in it as a "poyson". Despite the tremendous advances made in our chemical and toxicological capabilities (4) plus the lists of MSS toxicants, numerous noted critics of cigarette smoke have expressed reservation about the effect on the smoker of many of the MSS components listed as toxicants. Table 19 provides qualifying statements made not only by HOFFMANN and HECHT on several listed toxicants in the text accompanying their famous "List of 43" (7) but also by others on the biological activity of MSS toxicants.

\section{CONCLUSIONS}

- In terms of developing a "less hazardous" cigarette (LHC), one needs to define the reference point. If we compare commercial brands of today's cigarettes with those in the marketplace during the $1950 \mathrm{~s}$, then there is no question that LHCs have already been produced. However, the more important question is whether or not we can in the future develop LHCs than those in the marketplace today. This is the challenge facing the Tobacco Industry. We are optimistic that this goal can be achieved.

- Paramount among the criteria for new products is consumer acceptability. Regardless of the means to produce 
Table 19. Comments by various authorities on listed MSS toxicants

\begin{tabular}{|c|c|c|c|}
\hline MSS component & CAS no. & Comment & References \\
\hline \multirow[t]{4}{*}{ Benzo[a]pyrene } & $50-32-8$ & $\begin{array}{l}{[T] \text { he tarry condensates of the smoke obtained by smoking cigarettes }} \\
\text { in machines ... have } 3,4 \text {-benzpyrene but the amount is exceedingly } \\
\text { small and there is considerable doubt about whether the concentration } \\
\text { is high enough to produce carcinogenic action. }\end{array}$ & Cook $^{\text {a }}(275)$ \\
\hline & & $\begin{array}{l}\text { Whether it's benzo[a]pyrene or not, nobody really knows. More work } \\
\text { has been done on benzo[a]pyrene to prove it to be the causative agent } \\
\text { in cigarette smoking than I think on any other chemical for any disease } \\
\text { that I know. And yet the point is, you can't prove it. }\end{array}$ & Coultson (276) \\
\hline & & $\begin{array}{l}\text { But } 30 \text { years of laboratory research has yet to identify reliably the } \\
\text { important carcinogenic factors in cigarette smoke. }\end{array}$ & Peto and Doll ${ }^{\mathrm{b}}(277)$ \\
\hline & & $\begin{array}{l}\text { This complexity [of tobacco smoke] has made it difficult to identify any } \\
\text { individual agent within tobacco smoke as the chief cause of any of the } \\
\text { diseases that are caused by smoking... }\end{array}$ & IARC (44) \\
\hline \multirow[t]{2}{*}{ NNK } & $64091-91-4$ & It $[N N K]$ has not been tested by inhalation. & Hoffmann and Hecht (7) \\
\hline & & Relevant information not available [on this compound]. & OSHA (278) \\
\hline Aniline, 2-methyl- & $95-53-4$ & $\begin{array}{l}\text { Recent studies have ... shown that single ring aromatic amines, } \\
\text { including the weak bladder carcinogen } 0 \text {-toluidine [2-toluidine, } 2 \text { - } \\
\text { methylaniline] are present in human urine ... The available data do not } \\
\text { indicate that there are significant differences between smokers and } \\
\text { nonsmokers. }\end{array}$ & Hoffmann and Hecht (7) \\
\hline
\end{tabular}

Naphthalene, 2-amino- 91-59-8 2-[N]aphthylamine [has] been reported in tobacco or tobacco smoke. [That] compound is a bladder carcinogen in man ...., but is present in cigarette smoke in amounts ( $22 \mathrm{ng} /$ cigarette) too low to be considered a health hazard.

The presence of $\beta$-naphthylamine [2-aminonaphthalene] in cigarette smoke has been demonstrated . . ., along with other carcinogenic aromatic amines ... The yield is so low that the [the researchers] did not believe these agents contributed to the risk of bladder cancer in smokers.

On the basis of quantitative data for aromatic amines in cigarette smoke, an etiological significance of these traces of carcinogenic amines in bladder cancer is questionable...

Schmeltz and Hoffmann (279)

USPHS [see p. 41 in

Benzene

71-43-2 Concern has been expressed in recent years about the possible risk of USPHS [see p. 51 leukemia for workers who have been exposed to benzene . . . Although in (43)] some prospective and retrospective studies have reported a somewhat higher risk of leukemia for cigarette smokers, these data remain unconfirmed and no dose-response relationship has been established between death rate for leukemia and number of cigarettes smoked.

Acrylonitrile

75-05-8 Although it is present in cigarette [MSS], its role in tobacco

Hoffmann and Hecht (7) carcinogenesis is difficult to evaluate due to lack of data.

Vinyl chloride

75-01-4 Its low levels in cigarette [MSS] do not support a major role in tobacco Hoffmann and Hecht (7) carcinogenesis

Cadmium

Chromium

Lead

Nickel

440-43-9 The possible roles of chromium, cadmium, and lead in tobacco

Hoffmann and Hecht (7)

7440-47-4 carcinogenesis are difficult to evaluate given the present data base ...

7440-47-4 Taken together, the evidence for a major role of these materials as

7439-92-1 etiologic factors in tobacco carcinogenesis is not compelling.

7440-02-0 It is not likely that nickel plays a significant role in the etiology of lung cancer in cigarette smokers.

Polonium-210

7440-08-6 The quantities of polonium-210 found in the lungs of smokers are generally about three times higher than those in nonsmokers. However, the significance of polonium-210 in tobacco-induced lung cancer has been questioned upon comparison of these data with those obtained in miners.

In the case of polonium-210, a recent indepth [sic] study raises doubts USPHS [see p. 94 on the significance of ${ }^{210} \mathrm{Po}$ as a factor contributing to lung cancer in in (43)] smokers.

[P]olonium-210 is present in tobacco and tobacco smoke ( 0.03 to 1.0 $\mathrm{pCi} /$ cigarette); however, it is unlikely that these traces represent a major risk for the smoker.

USPHS [see p. 200 in

Hoffmann and Hecht (7); Harley et al. (77)

USPHS [see p. 211 in (172)]

\footnotetext{
${ }^{a}$ Cook and his colleagues isolated benzo[a]pyrene from coal tar, identified it, and demonstrated its carcinogenicity to mouse skin (280).

${ }^{\mathrm{b}}$ Doll was the author of one of the 1950 retrospective studies on smoking and lung cancer (2).
} 
LHCs, if people will not smoke them, then the effort is useless.

- For most of the last century, the chemistry of tobacco and its smoke has been at the forefront of developing improved smoking products; however, this appears to be changing. The analogy of the forest and trees seems to fit this situation. With chemistry, we can only look at individual MSS or groups of MSS constituents, i.e., the trees, at a time while biological assays within their limitations survey a broad range of effects, i.e., the forest. Ultimately we are not concerned with the health effects related to exposure to individual chemicals in smoke but rather to their effects as a mixture. It is beyond the scope of today's knowledge to predict the toxicology of a complex mixture from data on its individual components.

- Results of MSS chemical studies may be used to identify toxicants of concern and they can also quantify the amounts these constituents. However, neither the toxicology of the individual chemicals nor whether they have been reduced can be the basis for a "less hazardous" claim. Many scientists will claim that if we removed all the PAHs and TSNAs from MSS then a LHC would result. This is a true statement on an absolute and product stewardship basis, but whether it will make a meaningful improvement is unknown.

- Although approximately 4800 components of MSS are known, there are toxicological data on only a few hundred of these chemicals. Because of this great unknown, the Tobacco Industry's course of reducing MSS "tar" has been a prudent action.

- Scientists have hypothesized and there has been much public hoopla over the potential detrimental effects of tobacco additives and their pyrolysis products. However, we are not aware of a single scientific study that confirms the alleged adverse effects. To the contrary, there are several excellent, comprehensive studies that fail to demonstrate toxicological problems with tobacco additives.

- Reduction of vapor-phase constituents, e.g., carbon monoxide, has also been successful, but it appears that the great challenge especially to western countries is to develop consumer acceptable charcoal-filtered cigarettes or their equivalent. Many of the MSS toxicants ranking high in our quantitative risk assessments, e.g., acrolein, VNNAs, can be effectively removed by selective filtration. Some prominent scientists have hypothesized that a major factor influencing the differential lung cancer rate between Japanese and western smokers is the great popularity of charcoalfiltered cigarettes in Japan. This appears to be a situation where the MSS chemistry, biological assays, and epidemiological studies are in agreement, i.e., reducing MSS vapor-phase toxicants is beneficial.

- The modification of flue-curing barns from direct heating to heat exchanging appears to be a simple method to reduce TSNAs in flue-cured tobacco. Two questions arise from this change: 1) Whose idea was it to use direct-fired flue-curing barns and do they have any liability for creating a "more hazardous" cigarette, and 2) Does reducing the TSNAs make any biologically significant difference? The existing data do not support the contention that reducing TSNAs makes a meaningful difference. Of course, if this last statement be correct, then the answer to the first question is moot.

- Among the most intriguing research that we have encountered during this review is the seemingly beneficial effects of removing protein from tobacco. Perhaps the commercialization of deproteinized smoking tobacco is beyond the realm of feasibility, but with all the tools of modern agricultural science to produce custom-made crops, it would appear to be a fertile field of research.

\section{ACKNOWLEDGMENTS}

The authors are indebted to Dr. William S. Rickert of Labstat International, Inc. for providing analytical data on 1R4F cigarettes and Dr. Raymond C. Long of North Carolina State University for providing historical analytical data on DDT residues in US commercial cigarette blends. We also express our deep appreciation to Ms. Helen S. Chung, Ms. Patricia C. Comer and others at RJRT Science Information for their capable assistance with acquisition of numerous references. Additionally, we are grateful for the assistance of Dr. Kristen Green Jordan for proofreading parts of the manuscript.

\section{GLOSSARY}

\section{Abbreviations}

\begin{tabular}{|c|c|}
\hline 1R4F & $=$ Kentucky Reference Cigarette \\
\hline $\mathrm{A} \alpha \mathrm{C}$ & $=2$-amino- $9 H$-pyrido $[2,3-b]$ indole \\
\hline $\mathrm{ADC}$ & $=$ average daily concentration \\
\hline ACGIH & $\begin{aligned}= & \text { American Conference of Governmental } \\
& \text { Industrial Hygienists, Inc. }\end{aligned}$ \\
\hline ADI & $=$ acceptable daily intake \\
\hline $\mathrm{AEF}$ & $=$ aqueous ethanol-soluble fraction \\
\hline AM & $=$ antimutagenicity test \\
\hline $\mathrm{As}_{2} \mathrm{O}_{3}$ & $=$ arsenious oxide; arsenic trioxide \\
\hline AT & $=$ antitumorigenicity test \\
\hline $\mathrm{B} a \mathrm{~A}$ & $=$ benz $[a]$ anthracene \\
\hline $\mathrm{B} a \mathrm{P}$ & $=$ benzo $[a]$ pyrene \\
\hline $\mathrm{B} e \mathrm{P}$ & $=$ benzo $[e]$ pyrene \\
\hline CA & $=$ cellulose acetate \\
\hline CAS & $=$ Chemical Abstract Service \\
\hline $\mathrm{CCHE}$ & $\begin{aligned} &= \text { Center for Children's Health and Environ- } \\
& \text { ment }\end{aligned}$ \\
\hline CDD & $=$ chlorodibenzo- $p$-dioxin \\
\hline $\mathrm{CDF}$ & $=$ chlorodibenzofuran \\
\hline $\mathrm{CO}$ & $=$ carbon monoxide \\
\hline CPDB & $=$ Carcinogenic Potency Database \\
\hline CPSC & $=$ Consumer Product Safety Commission \\
\hline $\mathrm{CSC}$ & $=$ cigarette smoke condensate \\
\hline CORESTA & $\begin{aligned}= & \text { Centre de Coopération pour les Recherches } \\
& \text { Scientifiques relatives au Tabac }\end{aligned}$ \\
\hline DBA & $=\operatorname{dibenz}[a, h]$ anthracene \\
\hline $\mathrm{DB} a, i \mathrm{P}$ & $=$ dibenzo $[a, i]$ pyrene $=$ benzo $[r s t]$ pentaphene \\
\hline DDE, $p, p{ }^{\prime}-$ & $=1,1$-dichloro-2,2-bis( $p$-chlorophenyl)ethene \\
\hline DDT, $p, p{ }^{\prime}-$ & $=1,1,1$-trichloro-2,2-bis( $p$-chlorophenyl)ethane \\
\hline DHHS & $=$ Department of Health and Human Services \\
\hline DMBA & $=7,12$-dimethylbenz $[a]$ anthracene \\
\hline DMH & $=$ dimethylhydrazine \\
\hline
\end{tabular}




\begin{tabular}{|c|c|c|c|}
\hline $\begin{array}{l}\text { DNA } \\
\text { EC }\end{array}$ & $\begin{array}{l}=\text { deoxyribonucleic acid } \\
=\text { ethyl carbamate }\end{array}$ & NNAL & $\begin{aligned}= & 4-(N \text {-methylnitrosamino })-1-(3-\text { pyridinyl })-1- \\
& \text { butanol }\end{aligned}$ \\
\hline EPA & $=$ Environmental Protection Agency & NNK & $=4-(N-$ methylnitrosamino $)-1-(3-$ pyridinyl $)-1-$ \\
\hline \multirow[t]{2}{*}{ EPCRA } & $=$ Emergency Planning and Community & & butanone \\
\hline & Right-to-Know Act & NNN & $=N^{\prime}$-nitrosonornicotine \\
\hline \multirow[t]{2}{*}{ ERGO } & $=$ ERGO Forschungsgesellschaft $\mathrm{mbH}$, & NO & $=$ nitric oxide \\
\hline & Hamburg & NPRO & $=N$-nitrosoproline \\
\hline ETS & $=$ environmental tobacco smoke & NPYR & $=N$-nitrosopyrrolidine \\
\hline FTC & $=$ Federal Trade Commission & NSM & $=$ New Smoking Material \\
\hline GRAS & $=$ generally recognized as safe & ORNL & $=$ Oak Ridge National Laboratory \\
\hline Glu-P-1 & $\begin{aligned}= & 2 \text {-amino-6-methyldipyrido }\left[1,2-a: 3^{\prime}, 2^{\prime}-\right. \\
& d] \text { imidazole }\end{aligned}$ & OSHA & $\begin{array}{l}=\text { Occupational Safety and Health Admini- } \\
\text { stration }\end{array}$ \\
\hline Glu-P-2 & $=2$-aminodipyrido $\left[1,2-a: 3^{\prime}, 2^{\prime}-d\right]$ imidazole & $\mathrm{PAH}$ & $=$ polycyclic aromatic hydrocarbon \\
\hline $\mathrm{HCl}$ & $=$ hydrochloride & PCB & $=$ polychlorinated biphenyl \\
\hline $\mathrm{HCN}$ & $=$ hydrogen cyanide & PCDD & $=$ polychlorodibenzo- $p$-dioxin \\
\hline HEAST & $=$ Health Effects Summary Table & PCDF & $=$ polychlorodibenzofuran \\
\hline HERP & $=$ Human Exposure to Rodent Potential & $\mathrm{pCi}$ & $=$ picocurie \\
\hline $\mathrm{HI}$ & $=$ hazard index & PEL & $=$ permissible exposure level \\
\hline HTML & $=$ hypertext markup language & $\mathrm{PhIP}$ & $=2$-amino- $1-$ methyl- 6 -phenyl- $1 H$-imida- \\
\hline \multirow[t]{2}{*}{ IARC } & $=$ International Agency for Research on & & zo $[4,5-b]$ pyridine \\
\hline & Cancer & PHS & $=$ Public Health Service \\
\hline ILCR & $=$ incremental lifetime cancer risk & PM & $=$ Philip Morris or particulate matter \\
\hline \multirow[t]{2}{*}{ INBIFO } & $=$ INBIFO Institut für Biologische Forschung, & p.o. & $=$ per os (by mouth) \\
\hline & Köln, Germany & ${ }^{210} \mathrm{Po}$ & $=$ polonium -210 \\
\hline i.p. & $=$ intraperitoneal injection & $\mathrm{PP}$ & $=$ particulate phase \\
\hline \multirow[t]{2}{*}{ IQ } & $=2$-amino-3-methyl-3H-imidazo[4,5-f]quino- & $\mathrm{R}$ & $=$ rat \\
\hline & line & RAIS & $=$ Risk Assessment Information System \\
\hline IRIS & $=$ Integrated Risk Information System & $\mathrm{RfC}$ & $=$ reference concentration \\
\hline ISO & $=$ International Standards Organization & RJR, RJRT & = R.J. Reynolds Tobacco Co. \\
\hline IUPAC & $\begin{array}{l}=\text { International Union of Pure and Applied } \\
\text { Chemistry }\end{array}$ & RTECS & $\begin{array}{l}=\text { Registry of Toxic Effects of Chemical Sub- } \\
\text { stances }\end{array}$ \\
\hline IURF & $=$ Inhalation Unit Risk Factor & RTS & $=$ reconstituted tobacco sheet \\
\hline LHC & $=$ "less hazardous" cigarette & S-9 & $=\mathrm{a}$ rat liver homogenate fraction used to en- \\
\hline M & $=$ mouse & & hance mutagenesis detection \\
\hline 7-MBA & $=7-$ methylbenz $[a]$ anthracene & s.c. & $=$ subcutaneous injection \\
\hline $12-\mathrm{MBA}$ & $=12$-methylbenz $[a]$ anthracene & SSM & $=$ Sutton Smoking Material \\
\hline \multirow[t]{2}{*}{$\mathrm{MC}$} & $=3$-methylcholanthrene & SSS & $=$ sidestream smoke \\
\hline & $=1,2$-dihydro-3-methylbenz $[j]$ aceanthrylene & STEL & $=$ short-term exposure limit \\
\hline $\mathrm{MeA} \alpha \mathrm{C}$ & $=2$-amino-3-methyl- $9 H$-pyrido $[2,3-b]$ indole & TA98 & $=$ Salmonella typhimurium strain \\
\hline 5-MeC & $=5$-methylchrysene & TA1538 & $=$ Salmonella typhimurium strain \\
\hline \multirow[t]{2}{*}{ MeIQ } & $=2$-amino-3,4-dimethyl-3H-imidazo[4,5- & TBA & $=$ tumor-bearing animal \\
\hline & $f$ lquinoline & TCCD & $=2,3,7,8$-tetrachlorodibenzo- $p$-dioxin \\
\hline MeIQx & $\begin{aligned}= & 2 \text {-amino-3,8-dimethylimidazo }[4,5-f] \text { qui- } \\
& \text { noxaline }\end{aligned}$ & $\begin{array}{l}\mathrm{TD}_{50} \\
\mathrm{TDE}, p, p^{\prime}-\end{array}$ & $\begin{array}{l}=\text { tumor development in } 50 \% \text { of animals tested } \\
=1,1 \text {-dichloro-2,2-bis }(p \text {-chlorophenyl)ethane }\end{array}$ \\
\hline MS & $=$ mainstream & TEQ & $=$ toxicity equivalent \\
\hline MSS & $=$ mainstream smoke & TLV & $=$ Threshold Limit Value \\
\hline MTD & $=$ maximum tolerated dose & TPM & $=$ total particulate matter \\
\hline NAAC & $=N$-nitrosamino acid & Trp-P-1 & $=3$-amino-1,4-dimethyl-5H-pyrido $[4,3-$ \\
\hline NAB & $=N^{\prime}$-nitrosoanabasine & & $b$ ]indole \\
\hline NAT & $=N^{\prime}$-nitrosoanatabine & Trp-P-2 & $=3$-amino-1-methyl-5H-pyrido[4,3-b]indole \\
\hline NATA & $=$ National-scale air toxics assessment & TSNA & $=$ tobacco-specific $N$-nitrosamine \\
\hline NCDENR & $\begin{array}{l}=\text { North Carolina Department of Environment } \\
\text { and Natural Resources }\end{array}$ & $\begin{array}{l}\text { TWA }_{8} \\
\text { TWG }\end{array}$ & $\begin{array}{l}=8 \text {-hour time weighted average } \\
=\text { Tobacco Working Group }\end{array}$ \\
\hline NCI & $=$ National Cancer Institute & UK & $=$ United Kingdom \\
\hline NDEA & $=N$-nitrosodiethylamine & URF & $=$ Unit Risk Factor \\
\hline NDELA & $=N$-nitrosodiethanolamine & US & $=$ United States \\
\hline NDMA & $=N$-nitrosodimethylamine & USA & $=$ United States of America \\
\hline NEMA & $=N$-nitrosoethylmethylamine & USDA & $=$ United States Department of Agriculture \\
\hline NIOSH & $\begin{array}{l}=\text { National Institute of Occupational Safety } \\
\text { and Health }\end{array}$ & $\begin{array}{l}\text { USPHS } \\
\text { VNNA }\end{array}$ & $\begin{array}{l}=\text { United States Public Health Service } \\
=\text { volatile } N \text {-nitrosamine }\end{array}$ \\
\hline NNA & $=N$-nitrosamine & VP & $=$ vapor phase \\
\hline iso-NNAC & $=4-(N$-methylnitrosamino $)-4-(3-$ pyridinyl $)$ bu- & WHO & $=$ World Health Organization \\
\hline & tyric acid & WTPM & $=$ wet total particulate matter \\
\hline
\end{tabular}




\section{REFERENCES}

1. NRC: Complex mixtures: Methods for in vivo toxicity testing; National Research Council Press, Washington, DC, 1988.

2. Wynder, E.L and E.A. Graham: Tobacco smoking as a possible etiologic factor in bronchiogenic carcinoma: A study of six hundred and eighty-four proved cases; J. Am. Med. Assoc. 143 (1950) 329-336; Doll, R. and A.B. Hill: Smoking and carcinoma of the lung. Preliminary report; Brit. Med. J. (1950) ii, 739-748.

3. Wynder, E.L., E.A. Graham, and A.B. Croninger: Experimental production of carcinoma with cigarette tar; Cancer Res. 13 (1953) 855-864.

4. Green, C.R. and A. Rodgman: The Tobacco Chemists' Research Conference: A half century forum for advances in analytical methodology of tobacco and its products; Rec. Adv. Tob. Sci. 22 (1996) 131-304.

5. Kosak, A.I.: The composition of tobacco smoke; Experientia 10 (1954) 69-71.

6. United States Public Health Service: Smoking and Health. Report of the Advisory Committee to the Surgeon General of the Public Health Service; DHEW Publ. No. (PHS) 1103, 1964.

7. Hoffmann, D. and S.S. Hecht: Advances in tobacco carcinogenesis; in: Chemical carcinogenesis and mutagenesis. I, edited by C.S. Cooper and P.L. Grover, Springer-Verlag, London, UK, 1990, Chapter 3, pp. 63-102.

8. Environmental Protection Agency: Health effects of passive smoking: Assessment of lung cancer in adults and respiratory disorders in children; Draft Document EPA/600/6-90/006A (May 1990).

9. Rodgman, A.: Environmental tobacco smoke; Regul. Toxicol. Pharmacol. 16 (1992) 223-244.

10. Baker, R.R. and C.J. Proctor: A smoke odyssey; CORESTA Smoke-Techno Meeting, Xian, China, Paper IG 1, 2001.

11. Hoffmann, D., I. Hoffmann, and K. El-Bayoumy: The less harmful cigarette: A controversial issue. A tribute to Ernst L. Wynder; Chem. Res. Toxicol. 14 (2001) 767-790.

12. Hoffmann, D. and I. Hoffmann: The changing cigarette: Chemical studies and bioassays; in: Risks associated with smoking cigarettes with low machine-measured yields of tar and nicotine, NCI Smoking and tobacco control, Monograph 13, edited by D.M. Burns and N.L. Benowitz, Bethesda, MD, Chapter 5, 2001, pp.159-191.

13. Smith, C.J., S.D. Livingston, and D.J. Doolittle: An international literature survey of "IARC Group 1 carcinogens" reported in mainstream smoke; Food Chem. Toxicol. 35 (1997) 1107-1130.

14. Smith, C.J., T.A. Perfetti, M.A. Rumple, A. Rodgman, and D.J. Doolittle: "IARC Group 2A carcinogens" reported in cigarette mainstream smoke; Food Chem. Toxicol. 39 (2000) 371-383.

15. Smith, C.J., T.A. Perfetti, M.A. Rumple, A. Rodgman, and D.J. Doolittle: "IARC Group 2B carcinogens" reported in cigarette mainstream smoke; Food Chem. Toxicol. 39 (2001) 183-205.

16. Rodgman, A.: Some studies of the effects of additives on cigarette mainstream smoke properties. I. Flavorants; Beitr. Tabakforsch. Int. 20 (2002) 83-103.
17. Gaworski, C.L., M.M. Dozier, J.M. Gerhart, L.H. Brennecke, C. Aranyi, and J.D. Heck: 13-Week inhalation toxicity of menthol cigarette smoke; Food Chem. Toxicol. 35 (1997) 683-692.

18. Gaworski, C.L., M.M. Dozier, J.D. Heck, J.M. Gerhart, N. Rajendran, R.M. David, L.H. Brennecke, and R. Morrisey: Toxicologic evaluation of flavor ingredients added to cigarette tobacco: 13 -Week inhalation exposures in rats; Inhal. Toxicol. 10 (1998) 357-381.

19. Gaworski, C.L., J.D. Heck, M.B. Bennett, and M.L. Wenk: Toxicologic evaluation of flavor ingredients added to cigarette tobacco: Skin painting bioassay of cigarette smoke condensate in SENCAR mice; Toxicology 139 (1999) 1-17.

20. Carmines, E.L.: Evaluation of the potential effects of ingredients added to cigarettes. Part 1: Cigarette design, testing approach, and review of results; Food Chem. Toxicol. 40 (2002) 77-91.

21. Rustemeier, K., R. Stabbert, H.J. Haussmann, E. Roemer, and E.L. Carmines: Evaluation of the potential effects of ingredients added to cigarettes. Part 2: Chemical composition of mainstream smoke; Food Chem. Toxicol. 40 (2002) 93-104.

22. Roemer, E., F.J. Tewes, T.J. Meisgen, D.J. Veltel, and E.L. Carmines: Evaluation of the potential effects of ingredients added to cigarettes. Part 3: In vitro genotoxicity and cytotoxicity; Food Chem. Toxicol. 40 (2002) 105-111.

23. Vanscheeuwijck, P.M., A. Teredesai, P.M. Terpstra, J. Verbeeck, P. Kuhl, B. Gerstenberg, S. Gebel, and E.L. Carmines: Evaluation of the potential effects of ingredients added to cigarettes. Part 4: Subchronic inhalation studies; Food Chem. Toxicol. 40 (2002) 113-131.

24. Webster's Tenth New Collegiate Dictionary; edited by F.C. Mish, Merriam-Webster, Inc. Publishers, Springfield, MA, 1993, p. 558.

25. Rickert, W.S. and M.J. Kaiserman: Development of a numerical index for expressing the relative toxicity of mainstream tobacco smoke based on chemical composition; 52 ${ }^{\text {nd }}$ Tobacco Science Research Conference, Program Booklet and Abstracts, Vol. 52, Paper No. 63, 1998, p. 44.

26. Rickert, W.S., J.C. Robinson, and E. Lawless: Limitations to potential uses for data based on the machine smoking of cigarettes; in: Independent Scientific Committee on smoking and health symposium. "Nicotine smoking and the low tar programme"; Oxford Press, London, England, 1988.

27. Kaiserman, M.J.: Light cigarettes: Use and beliefs of Canadian smokers; Tobacco free Canada, Second National Conference on Tobacco or Health, Ottawa, Ontario, Oct. 30 - Nov. 2, 1996.

28. The National Center for Tobacco-Free Kids: Do you know that cigarettes expose smokers to ammonia, arsenic and formaldehyde? see www.tobaccofreekids.org, 1998.

29. Saint-Jalm, Y.: The regulatory situation in Europe Scientific issues and CORESTA action; CORESTA Newsletter 3 (2002) 5-8.

30. R.J. Reynolds Tobacco Company: Chemical and biological studies: New cigarette prototypes that heat instead of burn tobacco; R.J. Reynolds Tobacco Company, Winston-Salem, NC, 1988. 
31. Vorhees, D.J., W. Heiger-Bernays, and M.D. McClean: Human health risk associated with cigarette smoke: The link between smoke constituents and additives; Menzie-Cura \& Associates, Chelmsford, MA, 1997.

32. Tricker, A.R.: Toxicology of tobacco-specific nitrosamines; Rec. Adv. Tob. Sci. 27 (2001) 75-102.

33. Fowles, J. and M. Bates: The chemical constituents in cigarettes and cigarette smoke: Priorities for harm reduction, A Report to the New Zealand Ministry of Health; March 2000.

34. Rodgman, A.: Studies of polycyclic aromatic hydrocarbons in cigarette mainstream smoke: Identification, tobacco precursors, control of levels: A review; Beitr. Tabakforsch. Int. 19 (2001) 361-379.

35. Gori, G.B. (Editor): Report No. 1. Toward less hazardous cigarettes. The first set of experimental cigarettes; DHEW Publ. No. (NIH) 76-905 (1976); Report No. 2. Toward less hazardous cigarettes. The second set of experimental cigarettes; DHEW Publ. No. (NIH) 761111; Report No. 3. Toward less hazardous cigarettes. The third set of experimental cigarettes; DHEW Publ. No. (NIH) 77-1280; Report No. 4. Toward less hazardous cigarettes. The fourth set of experimental cigarettes; DHEW Publ. (NIH) March (1980); National Cancer Institute: Report No. 5. Toward less hazardous cigarettes. Summary: Four skin painting bioassays using condensate from experimental cigarettes; DHEW Publ. (NIH) (September 1980).

36. Wynder, E.L.: Statement on lung cancer-cigarette smoking controversy: Chemical fractionation of cigarette smoke condensate; in: False and misleading advertising (Filter-tip cigarettes), Wynder reported on the studies of G.F Wright at the Hearing before Subcommittee on Government Operations (the Blatnik Hearings on Filtered Cigarettes) (July, 1957), pp. 63-114.

37. Baker, R.R.: The development and significance of standards for smoking-machine methodology; Beitr. Tabakforsch. Int. 20 (2002) 23-41.

38. IARC: Some $N$-nitroso compounds; in: IARC monographs on the evaluation of the carcinogenic risks of chemicals in humans; IARC, Lyon, France, IARC. Sci. Publ. No. 17, 1978.

39. Environmental Protection Agency: Maleic hydrazide: Notification of issuances of notice of intent to suspend pesticide registration; Fed. Reg. 46 (No. 179) (1981) 45999-46000.

40. Brunnemann, K.D. and D. Hoffmann: Assessment of the carcinogenic $N$-nitrosodiethanolamine in tobacco products and tobacco smoke; Carcinogenesis 2 (1981) 1123-1127.

41. Hoffmann, D., K.D. Brunnemann, J.D. Adams, and S.S. Hecht: Formation and analysis of $N$-nitrosamines in tobacco products and their endogenous formation in consumers; in: $N$-Nitroso compounds: Occurrence, biological effects and relationship to human cancer, edited by I.K. O'Neill, R.C. von Borstel, C.T. Miller, J. Long, and H. Bartsch, IARC, Lyon, France, IARC Sci. Publ. No. 57 (1984) 743-762.

42. Rodgman, A.: The chemical composition of environmental tobacco smoke: Some comments on the Occupational Safety and Health Administration's notice on 'Indoor Air Quality'; Document submitted to the
Occupational Safety and Health Administration, 5 August, 1994, pp. i-xiii, 1-172, see www.rjrtdocs.com $515923456-645 ; 515926645-6833$.

43. United States Public Health Service: Smoking and health. A report of the Surgeon General; DHEW Publ. No. (PHS) 79-50066 (1979), see Chapt. 14.

44. IARC: Chemistry and analysis of tobacco smoke; in: Evaluation of the carcinogenic risk of chemicals to humans: Tobacco smoking; IARC, Lyon, France, IARC Monograph 38 (1986) 83-126, 387-394, see p. 118.

45. Nesemann, E., R. Schröder, and F. Seehofer: Methoden zur quantitativen Bestimmung von Insektiziden in Tabak und Tabakrauch. I. Mitteilung: Zur Bestimmung von Organo-Chlor-Insektiziden [The quantitative determination of insecticides in tobacco and tobacco smoke. 1st Report: The determination of organochlorine insecticides]; Beitr. Tabakforsch. 4 (1968) 182-188.

46. Hoffmann, D., G. Rathkamp, and E.L. Wynder: Chemical studies on tobacco smoke. IX. Quantitative analysis of chlorinated hydrocarbon insecticides; Beitr. Tabakforsch. 5 (1969) 140-148.

47. Sheets, T.J. and R.B. Leidy: Influence of insecticides and nematicides on the chemistry of tobacco; Rec. Adv. Tob. Sci. 5 (1979) 83-131.

48. Tso, T.C.: Production, physiology, and biochemistry of tobacco plant; Ideals, Inc., Beltsville, MD, 1990, p. 151.

49. Sheets, T.J.: Pesticide residues on tobacco: Perceptions and realities; Rec. Adv. Tob. Sci. 17 (1991) 33-65.

50. Sheets, T.J., R.B. Leidy, P.L. Messick, J.W. Laws, J.S. Hayes, W.L. Jones, and S.A. Meyers: Pesticide residues in tobacco, tobacco products, and mainstream smoke; 13-16 Annual Reports of Fate of Pesticide Residues on Tobacco, Project NC 03699, NC Agricultural Research Service, NC State University, Raleigh, NC, 1985, 1986, 1987.

51. Guthrie, F.E., C.B. McCants, and H.G. Small: Arsenic content of commercial tobacco, 1917-1958; Tob. Sci. 3 (1959) 62-64.

52. Griffin, H.R., M.B. Hocking, and D.G. Lowery: Arsenic determination in tobacco by atomic absorption spectrometry; Anal. Chem. 47 (1975) 229-233.

53. Cogbill, E.C. and M.E. Hobbs: The transfer of metallic constituents to the mainstream smoke; Tob. Sci. 1 (1957) 68-73.

54. Guthrie, F.E.: The nature and significance of pesticide residues on tobacco and in tobacco smoke; Beitr. Tabakforsch. 4 (1968) 229-245.

55. Van Duuren, B.L., J.A. Bilbao, and C.A. Joseph: The carcinogenic nitrogen heterocycles in cigarette smoke condensate; J. Natl. Cancer Inst. 25 (1960) 53-61.

56. Candeli, A., D. Hoffmann, and E.L. Wynder: Unpublished 1963 data, cited in: E.L. Wynder and D. Hoffmann: Experimental tobacco carcinogenesis; Adv. Cancer Res. 8 (1964) 249-453, see pp. 323-333.

57. Wynder, E.L. and D. Hoffmann: Tobacco and tobacco smoke: Studies in experimental carcinogenesis; Academic Press, New York, NY, 1967.

58. Rodgman, A.: Tobacco smoke components; Beitr. Tabakforsch. Int. 18 (1998) 127-129.

59. Sasaki, T.A. and S.C. Moldoveanu: Determination of dibenzacridines in the particulate phase of cigarette smoke; Beitr. Tabakforsch. Int. 19 (2000) 25-31. 
60. Crummett, W.B.: Environmental chlorinated dioxins from combustion - the trace chemistries of fire hypothesis; in: Chlorinated dioxins and related compounds, edited by O. Hutzinger, R.W. Frei, E. Merian, and F. Pocchiari, Pergamon Press, New York, NY, 1982, pp. 253-264.

61. Muzo, H. and Y. Takizawa: Dioxins in cigarette smoke; Arch. Environ. Health 44 (1989) 171-174.

62. Ball, M., O. Päpke, and A. Lis: Polychlordibenzodioxine und Polychlordibenzofurane in Cigarettenrauch [Polychlorodibenzodioxins and polychlorodibenzofurans in cigarette smoke]; Beitr. Tabakforsch. Int. 14 (1990) 393-402.

63. Matsueda, T., H. Hirakawa, T. Lida, Y. Kurokawa, and Y. Ohsaki: Concentration of polychlorinated dibenzo$p$-dioxins and polychlorinated dibenzofurans in cigarette smoke; Proceedings of the Annual Meeting of the Japanese Society of Air Pollution (1991) 475.

64. Löfroth, G. and Y. Zebühr: Polychlorinated dibenzo- $p$ dioxins (PCDDs) and dibenzofurans (PCDFs) in mainstream and sidestream cigarette smoke; Bull. Environ. Contam. Toxicol. 48 (1992) 789-794.

65. Matsueda, T., Y. Kurokawa, M. Nakamura, S. Takada, and K. Fukamachi: Concentrations of PCDDs, PCDFs and coplanar PCBs in cigarettes from various countries; Organohalogen Compounds 20 (1994) 331-334.

66. Berra, Y.: The Yogi book; Workman Publishing, Inc., New York, NY, 1998, p. 48.

67. Annals of the New York Academy of Sciences: Living in a chemical world: Occupational and environmental significance of industrial carcinogens, edited by $\mathrm{C}$. Maltoni and I.J. Selikoff; Vol. 534, 1988.

68. Annals of the New York Academy of Sciences: Tobacco smoking and nutrition: Influence of nutrition on tobacco-associated health risks, edited by J.N. Dana and W.A. Pryor; Vol. 686, 1993.

69. Annals of the New York Academy of Sciences: Toxicology for the next millennium, edited by R.J. Isfort and J. Lederberg; Vol. 919, 2000.

70. See the American Conference of Governmental Industrial Hygienists, Inc. website at www.acgih.org/TLVStmnt.htm, accessed on May 24, 2002.

71. Public Broadcasting System: Kids and chemicals; Bill Moyer, May 10, 2002, accessed at www.pbs.org/now/resources/kind.html.

72. Jacobs, M.: The reputation battle; C\&EN, June 17, 2002, p. 3.

73. The Rockefeller Family Fund ads may be found at the following website: www.childenvironment.org.

74. Gold, L.S., T.H. Slone, and B.N. Ames: Overview of the carcinogenic potency database; in: Handbook of carcinogenic potency and genotoxicity databases, edited by L.S. Gold and E. Seiger, CRC Press, Boca Raton, FL, 1997, pp. 661-685.

75. Gold, L.S., T.H. Slone, and B.N. Ames: What do animal cancer tests tell us about human cancer risk: Overview of analyses of the carcinogenic potency database; Drug Metabolism Reviews 30 (1998) 359-404.

76. US Environmental Protection Agency: Report of the EPA review workshop on alpha ${ }_{2 u}$-globulin: Association with renal toxicity and neoplasia in the male rat; US EPA, Washington, DC (1991).

77. Harley, N.H., B.S. Cohen, and T.C. Tso: Polonium210. A questionable risk factor in smoking-related carcinogenesis; in: A safe cigarette? Banbury Report 3, edited by G.B. Gori and F.G. Bock, Cold Spring
Harbor Laboratory, Cold Spring Harbor, NY, 1980, pp. 93-104.

78. New York State Department of Health: Appendix D: Procedure for evaluating potential health risk for contaminants of concern; in: Public health assessment, General Motors (Central Foundry Division) Massena. St. Lawrence County, NY (1999), accessed on June 9, 2002 at the following Centers for Disease Control website: www.atsdr.cdc.gov/HAC/PHA/gmcentral/gen_p2.html.

79. LaVoie, E.J., S.S. Hecht, D. Hoffmann, and E.L. Wynder: The less harmful cigarette and tobacco smoke flavors; in: A safe cigarette? Banbury Report 3, edited by G.B. Gori and F.G. Bock, Cold Spring Harbor Laboratory, Cold Spring Harbor, NY, 1980, pp. 251-260.

80. Doull, J., J.P. Frawley, W.J. George, T.A. Loomis, R.A. Squire, and S.L. Taylor: Cigarette ingredients: A complete list and background; Covington and Burling, Washington, DC, April 12, 1994, see www.rjirtdocs.com $517941576-1600$.

81. Paschke, T., H.-J. Eberhardt, W.-D. Heller, and G. Scherer: Effects of ingredients added to cigarette tobacco on smoke composition and biological activity: A literature overview; $54^{\text {th }}$ Tobacco Science Research Conference, Program Booklet and Abstracts, Vol. 54, Paper No. 9, 2000, pp. 21-22; Paschke, T., W-D. Heller, and G. Scherer: Effects of ingredients on cigarette smoke composition and biological activity: A literature overview; Beitr. Tabakforsch. Int. 20 (2003)107-247.

82. Rodgman, A.: Some studies of the effects of additives on cigarette mainstream smoke properties. II. Casing materials and humectants; Beitr. Tabakforsch. Int. 20 (2002) 279-299.

83. Schmeltz, I., A. Wenger, D. Hoffmann, and T.C. Tso: Chemical studies on tobacco smoke. 53. Use of radioactive tobacco isolates for studying the formation of smoke components; J. Agr. Food Chem. 26 (1978) 234-239.

84. Bio-Research Laboratories Ltd.: A comparative study of the mutagenicity of tobacco smoke condensate. Research Report (Project No. 7071): Mutagenicity of cigarette smoke condensates (Cigarette smoke condensates from Camel, Winston, Salem, Now, and Vantage as manufactured, flavorants excluded, casing materials excluded, both flavorants and casing materials excluded), August 30, 1977, see www.rjrtdocs.com $501542614-2638$.

85. Schumacher, J.N., C.R. Green, F.W. Best, and M.P. Newell: Smoke composition. An extensive investigation of the water-soluble portion of cigarette smoke; J. Agr. Food Chem. 25 (1977) 310-320.

86. Hege, R.B. Jr: Gas chromatographic determination of humectants in smoke; RDM, 1979, No. 41, November 7, see www.rjurtdocs.com 510547333 -7339.

87. Gaworski, C.L., J.D. Heck, and N. Rajendran: Toxicologic evaluation of glycerine and propylene glycol added to cigarette tobacco: 13-Week inhalation studies in Fischer-344 rats; $53^{\text {rd }}$ Tobacco Science Research Conference, Program Booklet and Abstracts, Vol. 53, Paper No. 05, 1999, pp. 21-22; Heck, J.D., C.L. Gaworski, N. Rajendran, and R.L. Morrisey: Toxicological evaluation of humectants added to cigarette tobacco: 13-Week inhalation study of glycerin and propylene glycol in Fischer 344 rats; Inhal. Toxicol. 14 (2002) 1135-1152. 
88. Newell, M.P., P.H. Latimer Jr, and L.R. Haefele: The fate of menthol in cigarette smoke; $22^{\text {nd }}$ Tobacco Chemists' Research Conference, Program Booklet and Abstracts, Vol. 22, Paper No. 24, 1968, p. 18.

89. Jenkins, R.W. Jr, R.H. Newman, and M.K. Chavis: Cigarette smoke formation studies. II. Smoke distribution and mainstream pyrolytic composition of added ${ }^{14} \mathrm{C}$-menthol (U); Beitr. Tabakforsch. 5 (1970) 299-301.

90. Bock, F.G., G.E. Moore, and P.C. Clark: Carcinogenic activity of cigarette smoke condensate. II. Biological activity of refined tar from several brands of cigarettes; J. Natl. Cancer Inst. 34 (1965) 481-493.

91. Lloyd, R.A., C.W. Miller, D.L. Roberts, J.A. Giles, J.P. Dickerson, N.H. Nelson, C.E. Rix, and P.H. Ayers: Flue-cured tobacco flavor. I. Essence and essential oil components; CORESTA 1974 Symposium, Montreux, Switzerland; Tob. Sci. 20 (1976) 40-50.

92. Roberts, D.L. and W.A. Rohde: Isolation and identification of flavor components of burley tobacco; Tob. Sci. 16 (1972) 107-112.

93. Schumacher, J.N. and L.L. Vestal: Isolation and identification of some components of Turkish tobacco; Tob. Sci. 18 (1974) 43-47.

94. Schumacher, J.N.: Flavor composition of Maryland tobacco; Beitr. Tabakforsch. Int. 12 (1982) 271-278.

95. Cooper, P.J. and R.B. Hege: The oxidation of NO to $\mathrm{NO}_{2}$ in cigarette smoke; 32 nd Tobacco Chemists' Research Conference, Program Booklet and Abstracts, Vol. 32, Paper No. 34, 1978, p. 18.

96. Borland, C.D.R., A.T. Chamberlain, T.W. Higenbottom, R.W. Barber, and B.A. Thrush: A comparison between the rate of reaction of nitric oxide in the gas phase and in whole smoke; Beitr. Tabakforsch. Int. 13 (1985) 67-73.

97. Cueto, R. and W.A. Pryor: Cigarette smoke chemistry: Conversion of nitric oxide to nitrogen dioxide and reactions of nitrogen oxides with other smoke components as studied by Fourier transform infrared spectroscopy; Vibrational Spectroscopy 7 (1994) 97-111.

98. Vilcins, G. and J.O. Lephardt: Ageing process of cigarette smoke. Formation of methyl nitrite; Chem. and Ind. (London) (1974) 974-975.

99. Shear, M.J. and J. Leiter: Studies in carcinogenesis. XVI. Production of subcutaneous tumors in mice by miscellaneous polycyclic compounds; J. Natl. Cancer Inst. 2 (1941) 241-258.

100. Crabtree, H.G.: Influence of bromobenzene on the induction of skin tumors by 3,4-benzopyrene; Cancer Res. 4 (1944) 688-693; Influence of unsaturated dibasic acids on the induction of skin tumors by chemical carcinogens; Cancer Res. 5 (1945) 346-351; Some effects of aromatic hydrocarbons on sulfur metabolism and tumor induction in mice; Cancer Res. 6 (1946) 553-559; Anticarcinogenesis; Brit. Med. Bull. 4 (1947) 345-348.

101. Hartwell, J.L.: Survey of compounds which have been tested for carcinogenic activity; USPHS Publ. No. 149, Washington, DC (1947); USPHS Publ. No. $149,2^{\text {nd }}$ Edition, Washington, DC (1951).

102. Shubik, P. and J.L. Hartwell: Survey of compounds which have been tested for carcinogenic activity, Suppl. 1; USPHS Publ. No. 149 Washington, DC (1957); Suppl. 2; USPHS Publ. No. 149, Washington, DC (1969).

103. Steiner, P.E. and H.L. Falk: Summation and inhibition effects of weak and strong carcinogenic hydrocarbons, 1:2-benzanthracene, chrysene, 1:2:5:6-dibenzanthracene, and 20-methylcholanthrene; Cancer Res. 11 (1951) 56-63.

104. Dipple, A., R.C. Moschel, and C.A.H. Bigger: Polynuclear hydrocarbons; Chapter 2, in: Chemical carcinogens. Second edition, edited by C.E. Searle, American Chemical Society Monograph 182, American Chemical Society, Washington, DC, 1984, pp. 41-163.

105. Hill, W.T., D.W. Stanger, A. Pizzo, B Riegel, P. Shubik, and W.B. Wartman: Inhibition of 9,10dimethyl-1,2-benzanthracene skin carcinogenesis in mice by polycyclic hydrocarbons; Cancer Res. 11 (1951) 892-897.

106. Slaga, T.J., L. Jecker, W.M. Bracken, and C.E. Weeks: The effects of weak or non-carcinogenic polycyclic hydrocarbons on 7,12-dimethylbenz [a]anthracene and benzo $[a]$ pyrene; Cancer Lett. 7 (1979) 51-59.

107. DiGiovanni, J., T.J. Slaga, D.L. Berry, and M.R. Juchau: Inhibitory effects of environmental chemicals on polycyclic aromatic hydrocarbon carcinogenesis; in: Carcinogenesis. A comprehensive survey. Vol. 5, edited by T.J. Slaga, Raven Press, New York, NY, 1980, pp. 145-168.

108. Snook, M.E., R.F. Severson, R.F. Arrendale, H.C. Higman, and O.T. Chortyk: The identification of high molecular weight polynuclear aromatic hydrocarbons in a biologically active fraction of cigarette smoke condensate; Beitr. Tabakforsch. Int. 9 (1977) 79-101.

109. Williams, D., F.J. Wiebel, J.C. Leutz, and H.V. Gelboin: Effect of polycyclic hydrocarbons in vitro on aryl hydrocarbon (benzo[a]pyrene) hydroxylase; Biochem. Pharmacol. 20 (1971) 2130.

110. Hoffmann, D. and E.L. Wynder: Selective reduction of the tumorigenicity of tobacco smoke. Experimental approaches; in: Toward a less harmful cigarette, Proceedings of Workshop on Smoking and Health, September, 1967, edited by E.L. Wynder and D. Hoffmann, Natl. Cancer Inst. Monograph 28, Washington, DC, 1968, pp. 151-172.

111. Rodgman, A. and L.C. Cook: The analysis of cigarette smoke condensate. XIV. Polycyclic aromatic hydrocarbons; RJRT RDR, 1960, No. 20, May 26, see www.rjrtdocs.com $501008592-8660$.

112. Slaga, T.J. and R.K. Boutwell: Inhibition of the tumor-initiating ability of the potent carcinogen 7,12dimethylbenz $[a]$ anthracene by the weak tumor initiator 1,2,3,4-dibenzanthracene; Cancer Res. 37 (1977) 129-133.

113. Slaga, T.J., A. Viaje, S.G. Buty, and W.M. Bracken: Dibenz[ $a, c]$ anthracene: A potent inhibitor of skintumor initiation by 7,12-dimethylbenz[ $a$ ] anthracene; Res. Comm. Chem. Pathol. Pharmacol. 19 (1978) 477-483.

114. Rodgman, A.: A comparison of the chemical and physical properties of cigarette mainstream smoke (MS), 
cigarette sidestream smoke (SS), and environmental tobacco smoke (ETS); Document submitted to the Environmental Protection Agency, December, 1991. Revised version submitted, June 1992, pp. i-vii + 1117, see www.rirtdocs.com 508185686 -5809.

115. Eatough, D.J., L.D. Hansen, and E.A. Lewis: The chemical characterization of environmental tobacco smoke; in: Environmental tobacco smoke, edited by D.J. Ecobichon and J.M. Wu, Proc. International Symposium at McGill University, PQ, Canada, 1989, Lexington Books, D.C. Heath and Company, Lexington, MA, 1990, pp. 3-39.

116. Rodgman, A. and L.C. Cook: The composition of cigarette smoke. IV. $\alpha$-Tocopherol; Tob. Sci. 4 (1960) 7-8.

117. Rodgman, A. and L.C. Cook: The composition of cigarette smoke. XI. Heterocyclic nitrogen compounds from Turkish tobacco smoke; Tob. Sci. 6 (1962) 176-179.

118. Izard, C., J. LaCharpagne, and P. Testa: Sur l'activité biologique de divers condensats de fumée de cigarettes, revelée par les tests auxiniques [On the biological activity disclosed in cellular tests of different cigarette smoke condensates]; Compt. Rend. 262D (1966) 1859-1861.

119. Rowland, R.L., A. Rodgman, J.N. Schumacher, D.L. Roberts, L.C. Cook, and W.E. Walker Jr: Macrocyclic diterpene hydroxyethers from tobacco and cigarette smoke; J. Org. Chem. 29 (1964) 16-21.

120. Falk, H.L., P. Kotin, and S. Thompson: Inhibition of carcinogenesis. The effect of hydrocarbons and related compounds; Arch. Environ. Hlth. 9 (1964) 169-179.

121. Homburger, F., A. Treger, and E. Boger: Experimental studies on the inhibition of carcinogenesis by cigarette-smoke condensates and carcinogen-related substances; in: Toward a less harmful cigarette, edited by E.L. Wynder and D. Hoffmann, Natl. Cancer Inst. Monograph 28,Washington, DC, 1968, pp. 259-270.

122. Hoffman, H.E. and A.C. Griffin: Action of cigarette tar and smoke on chemically induced carcinogenesis; Texas Rep. Biol. Med. 16 (1958) 333-345.

123. Wynder, E.L. and D. Hoffmann: Experimental tobacco carcinogenesis; Adv. Cancer Res. 8 (1964) 249-453.

124. Kotin, P. and H.L. Falk: Atmospheric factors in pathogenesis of lung cancer; Adv. Cancer Res. 7 (1953) 475-514, see 489-490.

125. Wynder, E.L., and D. Hoffmann: Bioassays on the carcinogenicity of tobacco smoke condensate and air pollutants; Proc. Am. Assoc. Cancer Res. 4 (1) (1963) 73.

126. Wynder. E.L. and D. Hoffmann: Studies with the gaseous and particulate phase of tobacco smoke; Proc. Am. Assoc. Cancer Res. 3 (4) (1962) 373.

127. Rothwell, K. and J.K. Whitehead: A method for the concentration of basic polycyclic heterocyclic compounds and the separation of polycyclic aromatic hydrocarbons from cigarette smoke condensate; Chem. and Ind. (London) (1969) 1628-1630.

128. Snook, M.E., R.F. Arrendale, H.C. Higman, and O.T. Chortyk: Isolation of indoles and carbazoles from cigarette smoke condensate; Anal. Chem. 50: (1978) 88-90; Snook, M.E., P.J. Fortson, and O.T. Chortyk: Isolation and identification of aza-arenes of tobacco smoke; Beitr. Tabakforsch. Int. 11 (1981) 67-78.

129. Wynder, E.L. and D. Hoffmann: Biological and chemical studies of tobacco smoke condensate; Proc. Am. Assoc. Cancer Res. 3 (1961) 280.

130. Rodgman, A.: The analysis of cigarette smoke condensate. II. The pretreatment of Camel blend tobacco; RDR, 1956, No. 12, November 1, see www.rjitdocs.com 501008294-8336; The analysis of cigarette smoke condensate. III. Flue-cured tobacco; RDR, 1957, No. 4, March 14, see www.rjirtdocs.com $501008337-8377$.

131. Wynder, E.L. and D. Hoffmann: A study of tobacco carcinogenesis. VII. The role of higher polycyclic hydrocarbons; Cancer 12 (1959) 1079-1086.

132. Hoffmann, D. and E.L. Wynder: A study of air pollution carcinogens. II. The isolation and identification of polynuclear aromatic hydrocarbons from gasoline engine exhaust condensate; Cancer 15 (1962) 93-102.

133. Homburger, F. and A. Treger (1965): Effects of intravenous carcinogen and tobacco condensate injections upon the incidence of lung tumors in $\mathrm{A} / \mathrm{He}$ mice; in: Lung tumors in animals, edited by L. Severi, Division of Cancer Research, University of Perugia, Italy, 1965, pp. 527-536.

134. Wynder, E.L. and D. Hoffmann: Experimental tobacco carcinogenesis; Science 182 (1968) 862-871.

135. Slaga, T.J. and J. DiGiovanni: Inhibition of chemical carcinogenesis; Chapter 21, in: Chemical carcinogens. Second edition, edited by C.E. Searle, American Chemical Society Monograph 182, American Chemical Society, Washington, DC, 1984, pp. 1279-1321.

136. Grasso, P.: Carcinogens in food; Chapter 19, in: Chemical carcinogens. Second edition, edited by C.E. Searle, American Chemical Society Monograph 182, American Chemical Society, Washington, DC, 1984, pp. 1205-1239.

137. Hoffmann, D., A. Rivenson, F.L. Chung, and E.L. Wynder: Potential inhibitors of tobacco carcinogenesis; in: Tobacco smoking and nutrition: Influence of nutrition on tobacco-associated health risks, edited by J.N. Diana and W.A. Pryor, Ann. NY Acad. Sci. 686 (1993) 140-160.

138. Wynder, E.L., G.F Wright, and J. Lam: A study of tobacco carcinogenesis. VI. The role of precursors; Cancer 12 (1959) 1073-1078.

139. Rowland, R.L.: Flue-cured tobacco. III. Solanachromene and $\alpha$-tocopherol; J. Am. Chem. Soc. 80 (1958) 6130-6133.

140. Saito, Y., H. Takizawa, S. Konishi, D. Yoshida, and S. Mizusaki: Identification of cembratriene-4,6-diol as a antitumor-promoting agent from cigarette smoke condensate; Carcinogenesis 6 (1985) 1189-1194.

141. Clemo, G.R.: Some aspects of the chemistry of tobacco smoke. I; Tetrahedron 3 (1958) 168-174.

142. Kosak, A.I., P.D. Rosen, and J.S. Swinehart: Components of cigarette smoke; Acta Unio Internat. Contra Cancrum 15 (1959) 612.

143. Fay, J.R., L.R. Perry, L.A. Kanerva, C.C. Sigman, and C.T. Helmes: Inhibitors of chemical carcinogenesis; Document prepared in 1984, revised in 1985 
for Sci. Coordinator Environ. Cancer, National Cancer Institute, Bethesda, MD, 1985, pp. 1-96.

144. Wattenberg, L.W. and J.B. Coccia: Inhibition of 4methylnitrosamino-1-(3-pyridyl)-1-butanone carcinogenesis in mice by $D$-limonene and citrus fruit oils; Carcinogenesis 12 (1991) 115-117.

145. Homburger, F., A. Treger, and E. Boger: Inhibition of murine subcutaneous and intravenous benzopentaphene carcinogenesis by sweet orange oils and $D$ limonene; Oncology 25 (1971) 1-10.

146. Waddell, W. and C. Marlowe: Inhibition by alcohols of the localization of radioactive nitrosonornicotine in sites of tumor formation; Science 221 (1983) 51-52.

147. Farinati, F., Z. Zhou, J. Bellah, C.S. Liebers, and A.J. Garro: Effect of chronic ethanol consumption on activation of nitrosopyrrolidine to a mutagen by rat upper alimentary tract, lung and hepatic tissue; Drug Metab. Dispos. 13 (1985) 210-214.

148. Wattenberg, L.W.: Inhibitors of chemical carcinogens; in: Cancer: Achievements, challenges and prospects for the 1980's, edited by J.H. Burchenal, Grune and Stratton, New York, NY, 1981, pp. 517-539.

149. Takeda, K., S. Ukawa, and M. Mochizuki: Inhibition by fatty acids of direct mutagenicity of $N$-nitroso compounds; in: Relevance to human cancer of $\mathrm{N}$ nitroso compounds, tobacco and mycotoxins, edited by I.K. O'Neill, J. Chen, and H. Bartsch, IARC, Lyon, France, IARC Sci. Publ. No. 105 (1991) 558-563.

150. Chung, F.L., A. Juchatz, J. Vitarius, and S.S. Hecht: Effects of dietary compounds on $\alpha$-hydroxylation of $N$-nitrosopyrrolidine and $N$ '-nitrosonornicotine in rat target tissues; Cancer Res. 44 (1984) 2924.

151. Chung, F.L., A. Juchatz, J. Vitarius, B. Reiss, and S.S. Hecht: Inhibition of target tissue activation of $N$ '-nitrosonornicotine and $N$-nitrosopyrrolidine by dietary components; in: $\mathrm{N}$-Nitroso compounds: Occurrence, biological effects and relevance to human cancer, edited by I.K. O'Neill, R.C. von Borstel, C.T. Miller, J. Long, and H. Bartsch, IARC, Lyon, France, IARC Sci. Publ. No. 57 (1984) 797-804.

152. Van Duuren, B.L., A. Sivak, C. Katz, and S. Melchionne: Cigarette smoke carcinogenesis: Importance of tumor promoters; J. Natl. Cancer Inst. 47 (1971) 235-240.

153. Thompson, H.J.: Effect of deficiencies of selenium and vitamin $\mathrm{E}$ alone or in combination on the induction of mammary carcinogenesis by 1-methyl-1nitrosourea; Carcinogenesis 12 (1991) 2175-2179.

154. Teel, R. and A. Castonguay: Antimutagenic effects of polyphenolic compounds; Cancer Lett. 66 (1992) 107-113.

155. Chung, F.L., M.A. Morse, K.I. Eklind, and Y. Xu: Inhibition of tobacco-specific nitrosamine-induced lung tumorigenesis by compounds derived from cruciferous vegetables and green tea; in: Tobacco smoking and nutrition: Influence of nutrition on tobacco-associated health risks, edited by J.N. Diana and W.A. Pryor, Ann. NY Acad. Sci. 686 (1993) 186-202.
156. Schüller, H.M., A. Castonguay, M. Orloff, and G. Rossignol: Modulation of the uptake and metabolism of 4-( $N$-methylnitrosamino)-1-(3-pyridyl)-1-butanone by nicotine; Cancer Res. 51 (1991) 2009-2114.

157. Lee, C.K., C.W. Fulp, D.W. Bombick, and D.J. Doolittle: Inhibition of mutagenicity of $\mathrm{N}$-nitrosamines by tobacco smoke and its constituents; Mutat. Res. 367 (1996) 83-92.

158. Brown, B., J.T. Avalos, C.K. Lee, and D.J. Doolittle: The effect of tobacco smoke, nicotine, and cotinine on the mutagenicity of 4-(methylnitrosamino)-1-(3pyridyl)-1-butanol (NNAL); $55^{\text {th }}$ Tobacco Science Research Conference, Program Booklet and Abstracts, Vol. 55, Paper No. 10, 2001, p. 26.

159. Berry, D.L., T.J. Slaga, J. DiGiovanni, and M.R. Juchau: Chlorinated dibenzo-p-dioxins, polybrominated biphenyls, and polychlorinated biphenyls in a two-stage system of mouse skin tumorigenesis: Potent anticarcinogenic effects; Ann. NY Acad. Sci. 320 (1979) 405-414.

160. Cohen, G.M., W.P. Bracken, R.P. Iyer, D.L. Berry, and T.J. Slaga: Anticarcinogenic effects of 2,3,7,8tetrachlorodibenzo- $p$-dioxin on benzo $[a]$ pyrene and 7,12-dimethylbenz $[a]$ anthracene tumor initiation and its relationship to DNA binding; Cancer Res. 39 (1979) 4027-4033.

161. DiGiovanni, J., T.J. Slaga, D.L. Berry, and M.R. Juchau: Inhibitory effects of environmental chemicals on polycyclic aromatic hydrocarbon carcinogenesis; in: Carcinogenesis. A comprehensive survey. Vol. 5, edited by T.J. Slaga, Raven Press, New York, NY, 1980, pp. 145-168; DiGiovanni, J.; D.L. Berry, G.L. Gleason, G.S. Kishore, and T.J. Slaga: Time-dependent inhibition by 2,3,7,8-tetrachlorodibenzo- $p$-dioxin of skin tumorigenesis with polycyclic hydrocarbons; Cancer Res. 40 (1980) 1580-1587.

162. Ramel, C., U.K. Alekperov, B.N. Ames, T. Kada, and L.W. Wattenberg: Inhibitors of mutagenesis and their relevance to carcinogenesis; Report by ICPEMC Expert Group on Antimutagens and Desmutagens; Mutat. Res. 168 (1986) 47-65.

163. Lee, C.K. and E.A. Reed: Ames test on smoke condensates: A summary; RJRT R\&DM, 1983, No. 20, May 9, see www.rjrtdocs.com $508352301-2303$.

164. Murphy, S.E. and R. Heilbrun: Effect of nicotine and tobacco-specific nitrosamines on the metabolism of $N$ '-nitrosonornicotine and 4-(methylnitrosamino)-1(3-pyridyl)-1-butanone by rat oral tissue; Carcinogenesis 11 (1990) 1663-1666.

165. Lee, C.K., J.A. Munoz, C.W. Fulp, K.M. Chang, J. Rogers, M.F. Borgerding, and D.J. Doolittle: Inhibitory activity of cigarette-smoke condensate on the mutagenicity of heterocyclic amines; Mutat. Res. 322 (1993) 21-32.

166. Sugimura, T.: Past, present, and future of mutagens in cooked foods; Environmental Hlth. Perspect. 67 (1986) 5-10.

167. Sugimura, T., T. Kawachi, M. Nagao, T. Yohagi, Y. Seino, T. Okamoto, K. Shudo, T. Kosuge, K. Tsuji, K. Watabayashi, Y. Iitaka, and A. Ita: Mutagenic principle(s) in tryptophan and phenylalanine pyrolysis products; Proc. Japan Acad. 53B (1977) 58-61; 
Sugimura, T., M. Nagao, T. Kawachi, M. Honda, T. Yahagi, Y. Seino, S. Sato, N. Matsukura, T. Matsushima, A. Shitai, M. Sawamura, and H. Matsumoto: Mutagens-carcinogens in food, with special reference to highly mutagenic pyrolytic products in broiled foods; in: Origins of human cancer, edited by H.H. Hiatt, J.D. Watson, and J.A. Winsten, Cold Spring Harbor Laboratory, Cold Spring Harbor, NY, 1977, pp. 1561-1577.

168. Yamamoto, T., K. Tsuji, T. Kosuge, T. Okamoto, K. Shudo, K. Takeda, Y. Iitaka, K. Yamaguchi, Y. Seino, T. Yahagi, M. Nagao, and T. Sugimura: Isolation and structure determination of mutagenic substances in $L$-glutamic acid pyrolysate; Proc. Japan Acad. 54B (1978) 248-250.

169. Yamashita, M., K. Wakabayashi, M. Nagao, S. Sato, and N. Kinea: Amounts of heterocyclic amines in the basic fraction of cigarette smoke condensates; Environ. Mutagen Soc. Japan Mtg., Akita, Japan: see Abstract in Mutat. Res. 164 (1986) 286; Yamashita, M., K. Wakabayashi, M. Nagao, S. Sato, Z. Yamaizumi, M. Takahashi, N. Kinea, I. Tomita, and T. Sugimura: Detection of 2-amino-3-methylimidazo[4,5-f]quinoline in cigarette smoke condensate; Gann 77 (1986) 419-422.

170. Felton, K.S. and M.G. Knize: Heterocyclic amine mutagens/carcinogens in foods; in: Chemical mutagenesis and carcinogenesis, edited by C.S. Cooper and P.L. Grover, Springer-Verlag, Berlin/Heidelberg: pp. 471-502.

171. Registry of Toxic Effects of Chemical Substances; 1985-1986 Edition User's Guide; DHHS Publ. No. (PHS) 87-114 (1987).

172. United States Public Health Service: The health consequences of smoking. Cancer. A report of the Surgeon General; DHHS Publ. No. (PHS) 82-50179 (1982).

173. Lavit-Lamy, D. and N.P. Buu-Hoï: The true nature of "dibenzo[a,l]pyrene" and its known derivatives; Chem. Comm. 4 (1966) 92-94.

174. Scherer, G.: Smoking behaviour and compensation: A review of the literature; Psychopharmacology 145 (1999) 1-20).

175. Baker, R.R. and L.S. Lewis: Filter ventilation - Has there been a "cover-up"?: Rec. Adv. Tob. Sci. 23 (1997) 152-196; The truth uncovered about filter vent blocking; CORESTA Congress, CORESTA Inf. Bull., Spec. Edition, 2000, Paper APST 4, p. 48; A review of the incidence and consequence of cigarette filter blocking among smokers; Beitr. Tabakforsch. Int. 19 (2001) 209-228.

176. Rodgman, A., C.J. Smith, and T.A. Perfetti: The composition of cigarette smoke: A retrospective, with emphasis on polycyclic components; Human Exptl. Toxicol. 19 (2000) 573-595.

177. Wynder, E.L. and G. F Wright: A study of tobacco carcinogenesis. I. The primary fractions; Cancer 10 (1957) 255-271.

178. Druckrey, H.: Experimental investigations on the possible carcinogenic effects of tobacco smoking; Acta Med. Scand. Suppl. 369 (1961) 24-42; Wynder, E.L. and D. Hoffmann: Present status of laboratory studies on tobacco carcinogenesis; Acta Path. Microbiol. Scand. 52 (1961) 119-132.

179. Roe, F.J.C.: The role of 3,4-benzopyrene in carcinogenesis by tobacco smoke condensate; Nature 194 (1962) 1089-1090; Acta Unio Internat. Contra Cancrum 19 (1963) 730.

180. Lazar, P.H., I. Chouroulinkov, C. Libermann, and M. Guerin: Amounts of 3,4-benzpyrene $(3,4-\mathrm{BP})$ in cigarette smoke condensates and carcinogenicity; $9^{\text {th }}$ Internat. Cancer Cong., Tokyo, Japan (1966); Benzo[a]pyrene content and carcinogenicity of cigarette smoke condensate: Results of short-term and longterm tests; J. Natl. Cancer Inst. 37 (1966) 573-579.

181. Wynder, E.L. and D. Hoffmann: Experimental tobacco carcinogenesis; Science 162 (1968) 862-871; A study of tobacco carcinogenesis. X. Tumor promoting activity; Cancer 24 (1969) 289-301.

182. Kensler, C.J. and S.P. Battista: Components of cigarette smoke with ciliary-depressant activity: Their selective removal by filters containing activated charcoal granules; New Eng. J. Med. 269 (1963) 1161-1166.

183. Dalhamn, T., M.L. Edfors, and R. Rylander: Mouth absorption of various compounds in cigarette smoke; Arch. Environ. Hlth. 16 (1968) 831-835; Retention of cigarette smoke components in human lungs; Arch. Environ. Hlth. 17 (1968) 746-748.

184. Boyland, E., F.J.C. Roe, and J.W. Gorrod: Induction of pulmonary tumours in mice by nitrosonornicotine, a possible constituent of tobacco smoke; Nature 202 (1964) 1126.

185. Boyland, E., F.J.C. Roe, J.W. Gorrod, and B.V.C. Mitchley: The carcinogenicity of nitrosoanabasine, a possible constituent of tobacco smoke; Brit. J. Cancer 18 (1964) 265-272; Roe, F.J.C., E. Boyland, and J.W. Gorrod: The importance of looking for further carcinogens in tobacco smoke, and the possible role of nitrosoanabasine; in: Compounds having alkylating action, Verband der Cigarettenindustrie, Hamburg, West Germany, 1964, pp. 85-92.

186. Searle, C.E. (editor): Chemical carcinogens. Second edition, American Chemical Society Monograph 182, American Chemical Society, Washington, DC, 1984.

187. Preussmann, R. and B.W. Stewart: $N$-Nitroso carcinogens; Chapter 12, in: Chemical carcinogens. Second edition, edited by C.E. Searle, American Chemical Society Monograph 182, American Chemical Society, Washington, DC, 1984, pp. 643-828.

188. Brune, H. and S. Henning: Erzeugung von Augenlidcarcinomen bei Mäusen nach epicutaner Applikation von Methyl-butyl-nitrosamin [Production of eyelid carcinoma in mice after skin application of methylbutylnitrosamine]; Z. Krebsforsch. 69 (1967) 307-308.

189. Hoffmann, F. and A. Graffi: Carcinome der Nasenhöhle bei Mäusen nach Tropfung der Rückenhaut mit Diäthylnitrosamin [Nasal carcinomas in mice after treating the skin of the back with diethylnitrosamine]; Acta Biol. Med. German. 12 (1964) 623-625; Nasenhöhlentumoren bei Mäusen nach percutaner Diäthylnitrosaminapplikation [Nasal carcinomas in mice after skin application of diethylnitrosamine]; 
Arch. Geschwulstforsch. 23 (1964) 274-288.

190. Herrold, K.M.: Effect of route of administration on the carcinogenic action of diethylnitrosamine $(\mathrm{N}$ nitrosodiethylamine); Brit. J. Cancer 78 (1964) 189-195.

191. Hoffmann, D., A. Rivenson, J.D. Adams, A. Juchatz, N. Vinchkoski, and S.S. Hecht: Effects of route of administration and dose on the carcinogenicity of $\mathrm{N}$ nitrosodiethanolamine in the Syrian golden hamster; Cancer Res. 43 (1983) 2521-2524.

192. LaVoie, E.J., G. Prokopczyk, J. Rigotty, A. Czech, and A. Rivenson: Tumorigenic activity of the tobacco-specific nitrosamines 4-(methylnitrosamino)1-(3-pyridyl)-1-butanone (NNK), 4-(methylnitrosamino)-1-(3-pyridyl)-1-butanol (iso-NNAL), and $N^{\prime}$ nitrosonornicotine on topical application to Sencar mice; Cancer Lett. 37 (1987) 277-283.

193. Deutsch-Wenzel, R.P., H. Brune, G. Grimmer, and J. Misfeld: Local application to mouse skin as a carcinogen specific test system for nonvolatile $N$-nitroso compounds; Cancer Lett. 29 (1985) 85-92.

194. Neurath, G., B. Pirmann, and H. Wichern: Zur Frage der $N$-Nitroso-Verbindungen im Tabakrauch [ $N$-Nitroso compounds in tobacco smoke]; Beitr. Tabakforsch. 2 (1964) 311-319.

195. Neurath, G., B. Pirmann, W. Lüttich, and H. Wichern: Zur Frage der $N$-Nitroso-Verbindungen im Tabakrauch. II [ $N$-Nitroso compounds in tobacco smoke. II]; Beitr. Tabakforsch. 3 (1965) 251-262.

196. Krull, J.S., T.Y. Fan, and D.H. Fine: Problem of artifacts in the analysis of $N$-nitroso compounds; Anal. Chem. 50 (1978) 698-701.

197. Eisenbrand, G., M. Archer, K.D. Brunnemann, D.H. Fine, S.S. Hecht, D. Hoffmann, J. Krull, and K.S. Webb: Problems of contamination and artefact formation in nitrosamine sampling and analysis; in: Environmental carcinogens. Selected methods of analysis. Vol. 6: $N$-Nitroso compounds, edited by H. Egan, R. Preussmann, G. Eisenbrand, T. Spiegelhalder, I.K. O'Neill, and H. Bartsch, IARC, Lyon, France, IARC Sci. Publ. No. 45 (1983) 25-34.

198. Caldwell, W.S. and J.M. Conner: Artifact formation during smoke trapping. An improved method for the determination of $\mathrm{N}$-nitrosamines in cigarette smoke; J. Assoc. Off. Anal. Chem. 73 (1990) 783-789.

199. Fredrickson, J.D.: Personal communication (1965/1967).

200. Morie, G.P. and C.H. Sloan: Determination of $N$ nitrosodimethylamine in the smoke of high-nitrate tobacco cigarettes; Beitr. Tabakforsch. 7 (1973) 61-66.

201. Brunnemann, K.D., L. Yu, and D. Hoffmann: Assessment of carcinogenic volatile $N$-nitrosamines in tobacco and mainstream and sidestream smoke from cigarettes; Cancer Res. 37 (1977) 3218-3222.

202. Hoffmann, D., G. Rathkamp, and Y.Y. Liu: Chemical studies on tobacco smoke. XXVI. On the isolation and identification of volatile and nonvolatile $N$-nitrosamines and hydrazines in cigarette smoke; in: $\mathrm{N}$ Nitroso compounds in the environment, edited by $\mathrm{P}$. Bogovski and E.A. Walker, IARC, Lyon, France, IARC Sci. Publ. No. 9 (1974) 159-165.

203. Wynder, E.L. and D. Hoffmann: Present status of laboratory studies on tobacco carcinogenesis; Acta Path. Microbiol. Scand. 52 (1961) 119-132.

204. Laurene, A.H., G.W. Young, and L.A. Lyerly: Factors which affect the phenol content of cigarette smoke; RDR, 1963, No. 58, November 13, see www.rjirtdocs.com 500962130-2163.

205. Hecht, S.S., C.B. Chen, N. Hirota, R.M. Ornaf, T.C. Tso, and D. Hoffmann: Tobacco specific nitrosamines: Formation from nicotine in vitro and during curing of tobacco and carcinogenicity in Strain-A mice; J. Natl. Cancer Inst. 60 (1978) 819-824.

206. Adams, J.D., S.J. Lee, N. Vinchkoski, A. Castonguay, and D. Hoffmann: [Chemical studies on tobacco smoke. LXXIII]. On the formation of the tobaccospecific carcinogen 4-(methylnitrosamino)-1-(3-pyridyl)-1-butanone during smoking; Cancer Lett. 17 (1983) 339-346.

207. Hoffmann, D., S.S. Hecht, R.M. Ornaf, E.L. Wynder, and T.C. Tso: Chemical studies on tobacco smoke. XLII. Nitrosonornicotine: Presence in tobacco, formation and carcinogenicity; in: Environmental $N$-nitrosamines: Analysis and formation, edited by E.A. Walter, P. Bogovski, and L. Griciute, IARC, Lyon, France, IARC Sci. Publ. No. 14 (1976) 307-320.

208. Hecht, S.S., J.D. Adams, and D. Hoffmann: Tobaccospecific nitrosamines in tobacco and tobacco smoke; in: Environmental carcinogens. Selected methods of analysis. Vol. 6: $\mathrm{N}$-Nitroso compounds; edited by $\mathrm{H}$. Egan, R. Preussmann, G. Eisenbrand, T. Spiegelhalder, I.K. O'Neill, and H. Bartsch, IARC, Lyon, France, IARC Sci. Publ. No. 45 (1983) 93-101.

209. Tso, T.C., J.L. Sims, and D.E. Johnson: Some agronomic factors affecting $N$-dimethylnitrosamine content in cigarette smoke; Beitr. Tabakforsch. 8 (1975) 34-38.

210. Fischer, S., B. Spiegelhalder, J. Eisenbarth, and R. Preussmann: Investigations on the origin of tobaccospecific nitrosamines in mainstream smoke of cigarettes; Carcinogenesis 11 (1990) 723-730.

211. Fischer, S., B. Spiegelhalder, and R. Preussmann: No pyrosynthesis of $N$ '-nitrosonornicotine (NNN) and 4( $N$-methylnitrosamino)-1-(3-pyridyl)-1-butanone (NNK) from nicotine; in: Effects of nicotine on biological systems, edited by F. Adlkofer and K. Thurau, Birkhauser Verlag, Boston, MA, 1991, pp. 103-107.

212. Castonguay, A.: Pulmonary carcinogenesis and its prevention by dietary polyphenolic compounds; in: Tobacco smoking and nutrition, edited by J.N. Diana and W.A. Pryor, Ann. NY Acad. Sci. 868 (1993) 177-185.

213. Renaud, J.M., S. d'Andres, R. Boudoux, and J. Zuber: TSNA levels in the mainstream smoke of simplified blend prototypes; CORESTA Smoke Technology Meeting, Xian, China (2001); d'Andres, S., R. Boudoux, J.-M. Renaud, and J. Zuber: TSNA levels in the mainstream smoke of simplified blend prototypes; Beitr. Tabakforsch. Int. 20 (2003) 331-340.

214. Moldoveanu, S.C., N.P. Kulshreshtha, and J.M. Wilkins: Study of the pyrosynthesis of NNN and NNK in mainstream cigarette smoke; $55^{\text {th }}$ Tobacco Science Research Conference, Program Booklet and Abstracts, 
Vol. 55, Paper No. 60, 2001, p. 55.

215. Haut, S.A.: The effect of ionic nitrate addition on mainstream TSNA delivery; Memorandum, November 19, 1990, see www.pmdocs.com 2024048764 -8771; Mainstream NO, TSNA, and filler nitrate; Memorandum, March 18, 1991, see www.pmdocs.com $2029088923-8927$.

216. Hecht, S.S. and D. Hoffmann: 4-(N-Methylnitrosamino)-1-(3-pyridyl)-1-butanone, a nicotine-derived tobacco-specific nitrosamine, and cancer of the lung and pancreas in humans; in: The origins of human cancer: A comprehensive review, edited by J. Brugge, T. Curran, E. Harlow, and F. McCormick, Cold Spring Harbor Laboratory, Cold Spring Harbor, NY, 1991, pp. 745-755.

217. Rickert, W.S. and W. Wright: Stability of yields of Canadian mandated analytes from the 1R4F Kentucky Reference Cigarette: A time series analysis; 2002 CORESTA Congress, New Orleans, LA.

218. Prokopczyk, B., A. Rivenson, and D. Hoffmann: Comparative carcinogenicity of 3-(methylnitrosamino)propionitrile and 4-(methylnitrosamino)-1-(3-pyridyl)-1-butanone upon local application to mouse skin and rat oral mucosa; Cancer Lett. 60 (1991) 153-157.

219. Rivenson, A., D. Hoffmann, B. Prokopczyk, S. Amin, and S.S. Hecht: Induction of lung and exocrine pancreas tumors in F344 rats by tobacco-specific and Areca-derived $N$-nitrosamines; Cancer Res. 48 (1988) 6912-6917.

220. Belinsky, S.A., J.F. Foley, C.M. White, M.W. Anderson, and R.R. Maronpot: Dose-response relationship between $\mathrm{O}^{6}$-methylguanine formation in Clara cells and induction of pulmonary neoplasia in the rat by 4-(methylnitrosamino)-1-(3-pyridyl)-1butanone; Cancer Res. 50 (1990) 3772-3780.

221. Castonguay, A. and N. Rioux: Inhibition of lung tumorigenesis by sulindac: Comparison of two protocols; Carcinogenesis 18 (1997) 491-496.

222. Peterson L.A. and S.S. Hecht: $\mathrm{O}^{6}$-Methylguanine is a critical determinant of 4-(methylnitrosamino)-1-(3pyridyl)-1-butanone tumorigenesis in $\mathrm{A} / \mathrm{J}$ mouse lung; Cancer Res. 51 (1991) 5557-5564.

223. Hecht, S.S., J.D. Adams, S. Numoto, and D. Hoffmann: Induction of respiratory tract tumors in Syrian golden hamsters by a single dose of 4-(methylnitrosamino)-1-(3-pyridyl)-1-butanone (NNK) and the effect of smoke inhalation; Carcinogenesis 4 (1983) 1287-1290.

224. Hoffmann, D., A. Rivenson, S. Amin, and S.S. Hecht: Dose response study of the carcinogenicity of tobacco-specific $N$-nitrosamines in F344 rats; J. Cancer Res. Clin. Oncol. 1008 (1984) 81-86.

225. Hoffmann, D., M.V. Djordjevic, A. Rivenson, D. Desai, and S. Amin: Relative potencies of tobaccospecific $N$-nitrosamines as inducers of lung tumors in A/J mice; Cancer Lett. 71 (1993) 25-30.

226. Gori, G.B.: A summary appraisal; in: A safe cigarette? Banbury Report 3, edited by G.B. Gori and F.G. Bock, Cold Spring Harbor Laboratory, Cold Spring Harbor, NY, 1980, pp. 353-359.

227. Shimkin, M.B.: Pulmonary tumors in experimental animals; Adv. Cancer Res. 3 (1955) 223-267, see p.

\section{7, Table I.}

228. P. Lorillard Company Research Laboratory: Selective filtration of phenol by Kent's improved Micronite filter; P. Lorillard Co., Greensboro, NC, 1-4 (1962).

229. Hoffmann, D. and E.L. Wynder: Die Filtration von Phenolen aus Cigarettenrauch [Adsorption capacity of cigarette filters for phenols from the smoke]; Beitr. Tabakforsch. 2 (1963) 51-66; Filtration of phenols from cigarette smoke; J. Natl. Cancer Inst. 30 (1963) 67-84.

230. Hoffmann, D. and E.L. Wynder: The reduction of the tumorigenicity of cigarette smoke condensate by addition of sodium nitrate to tobacco; Cancer Res. 27 (1967) 172-174.

231. Druckrey, H. and R. Preussmann: Zur Entstehung carcinogener Nitrosamine am Beispiel des Tabakrauchs [The possible formation of carcinogenic nitrosamines in tobacco smoke]; Naturwissenschaften 49 (1962) 498-499.

232. Wynder. E.L. and D. Hoffmann: Studies in tobacco carcinogenesis; Proc. Am. Assoc. Cancer Res. 3 (2) (1960) 164.

233. Wynder, E.L. and S.S. Hecht (Editors): Lung cancer; UICC Tech. Rept. Series 25 (1976) 138.

234. Wynder, E.L. and D. Hoffmann: Ein experimenteller Beitrag zur Tabakrauchkanzerogenese [An experimental contribution to tobacco smoke cancerogenesis]; Deut. Med. Wchnschr. 88 (1963) 623-628.

235. Bryant, H.G. Jr and V. Norman: Tobacco composition; US Patent No. 4,248,251 (February 3, 1981).

236. Kallianos, A.G., R.E. Means, and J.D. Mold: Effect of nitrates in tobacco on the catechol yield in cigarette smoke; Tob. Sci. 12 (1968) 125-129.

237. Brunnemann, K.D. and D. Hoffmann: [Chemical studies on tobacco smoke. LXXIV]. Pyrolytic origins of major gas phase constituents of cigarette smoke; Rec. Adv. Tob. Sci. 8 (1982) 103-140.

238. Brunnemann, K.D., J. Masaryk, and D. Hoffmann: Role of tobacco stems on the formation of $N$-nitrosamines in tobacco and cigarette mainstream and sidestream smoke; J. Agr. Food Chem. 31 (1983) 1221-1224.

239. Adams, J.D., S.J. Lee, and D. Hoffmann: Carcinogenic agents in cigarette smoke and the influence of nitrate on their formation; Carcinogenesis 5 (1984) 221-223.

240. Rathkamp, G., D. Hoffmann, and E.L. Wynder: Experiments on the reduction of polynuclear aromatic hydrocarbons in cigarette smoke; $20^{\text {th }}$ Tobacco Chemists' Research Conference, Program Booklet and Abstracts, Vol. 20, Paper No. 19 (1966), p. 23.

241. Rathkamp, G. and D. Hoffmann: Chemical studies on tobacco. XIII. The inhibition of the pyrosynthesis of several selective smoke components; Beitr. Tabakforsch. 5 (1970) 302-306.

242. Hoffmann, D., T.C. Tso and G.B. Gori: The less harmful cigarette; Preventive Med.9(1980) 287-296.

243. Hecht, S.S., R.M. Ornaf, and D. Hoffmann: $N$-Nitrosoalkaloids in tobacco; $28^{\text {th }}$ Tobacco Chemists' Research Conference, Program Booklet and Abstracts, Vol. 28, Paper No. 36, 1974, p. 25. 
244. Szent-Gyorgyi, A.: Removal of polycyclic aromatic hydrocarbons from cigarette mainstream smoke by chloranil; Personal communication to R.J. Reynolds Tobacco Company, 1960.

245. Dalhamn, T.: Some factors influencing the respiratory toxicity of cigarette smoke; J. Natl. Cancer Inst. 48 (1972) 1821-1824.

246. Wynder, E.L., P. Kopf, and H. Ziegler: A study of tobacco carcinogenesis. II. Dose-response studies; Cancer 10 (1957) 1193-1200.

247. Wynder, E.L. and D. Hoffmann: Some practical aspects of the smoking-cancer problem; New Eng. J. Med. 262 (1960) 540-545.

248. Wynder, E.L. and D. Hoffmann: Reduction of tumorigenicity of cigarette smoke. An experimental approach; J. Am. Med. Assoc. 192 (1965) 88-94.

249. Wynder, E.L. and D. Hoffmann: Current concepts of environmental cancer research; Med. Clin. N. America 50 (1966) 631-650.

250. Wynder, E.L. and D. Hoffmann: Bioassays in tobacco carcinogenesis; Prog. Exp. Tumor Res. 11 (1969) 163-193.

251. Wynder, E.L. and D. Hoffmann: Tobacco and health: A societal challenge; New Eng. J. Med. 300 (1979) 894-903.

252. Hoffmann, D., I. Schmeltz, S.S. Hecht, and E.L. Wynder: Polynuclear aromatic hydrocarbons in tobacco carcinogenesis; Chapter 3, in: Polycyclic hydrocarbons and cancer. Vol. 1, Chemistry, molecular biology and environment, edited by G. Gelboin and P.O. Ts'o, Academic Press, New York, NY, 1978, pp. 85-117.

253. United States Public Health Service: The health consequences of smoking. The changing cigarette. A report of the Surgeon General; DHHS Publ. No. (PHS) 81-50156 (1981) 51-52.

254. Hoffmann, D. and E.L. Wynder: Chemical constituents and bioactivity of tobacco smoke; in: Tobacco: A major health hazard, edited by D.G. Zardidze and R. Peto, IARC, Lyon, France, IARC Sci. Publ. No. 74 (1986) 145-165.

255. Hoffmann, D. and I. Hoffmann: [Chemical studies on tobacco smoke. C]. The changing cigarette: 1950-1995; J. Toxicol. Environ. Hlth. 50 (1997) 307-364.

256. Fredrickson, J.D.: Process for increasing the filling capacity of tobacco; US Patent No. 3,542,451 (August 18, 1970); Moser, G.P. and G.M. Stewart: Process for increasing the filling value of tobacco; US Patent No. 3,542,452 (August 18, 1970).

257. Weber, K.H.: Recent changes in tobacco products and their acceptance by the consumer; Proceedings $6^{\text {th }}$ International Tobacco Scientific Congress, Tokyo, Japan, 1976, pp. 47-63.

258. Hecht, S.S., A. Castonguay, A. Rivenson, B. Mu, and D. Hoffmann: Tobacco-specific nitrosamines: Carcinogenicity, metabolism, and possible role in human cancer; J. Environ. Hlth. Sci. 1 (1983) 1-54; Hecht, S.S., N.J. Haley, and D. Hoffmann: Monitoring exposure to tobacco products by measurement of nicotine metabolites and derived carcinogens; in: Molecular dosimetry and human cancer: Analytical, epidemiological and social considerations, edited by J.D. Groopman and P.L. Skipper, CRC Press, Boston, MA, 1991, pp. 325-361.

259. Hoffmann, D., K.D. Brunnemann, B. Prokopczyk, and M.V. Djordjevic: Tobacco-specific $N$-nitrosamines and Areca-derived $N$-nitrosamines: chemistry, biochemistry, carcinogenicity, and relevance to humans; J. Toxicol. Environ. Hlth. 41 (1994) 1-52.

260. Hoffmann, D., I. Hoffmann, and E.L. Wynder: Lung cancer and the changing cigarette; in: Relevance to human cancer of $N$-nitroso compounds, tobacco and mycotoxins, edited by I.K. O'Neill, J. Chen, and H. Bartsch, IARC, Lyon, France, IARC Sci. Publ. No. 105 (1991) 449-459; Hoffmann, D., M.V. Djordjevic, and I. Hoffmann: The changing cigarette; Prev. Med. 26 (1997) 427-434; Reference 11: 767-790.

261. Green, C.R., L. Vestal, and J.N. Schumacher: The investigation of the cigarette smoke from Celanese smoking material; RDR, 1969, No. 32, September 19, see www.rjrtdocs.com 500969764 -9795.

262. Williams J.R.: Method of treating tobacco to reduce nitrosamine content, and produce products thereby; US Patent 6,202,649 (March 20, 2001)

263. Peele, D.M., M.G. Riddick, M.E. Edwards, J.S. Gentry, and T.B. Nestor: Formation of tobaccospecific nitrosamines in flue-cured tobacco; Rec. Adv. Tob. Sci. 27 (2001) 3-12.

264. Doolittle, D.J., J.T. Avalos, B.R. Bombick, K.P. Putnam, D.W. Bombick, T.B. Nestor, and J.S. Gentry: Biological studies on smoke condensates from cigarettes made with low nitrosamine flue-cured tobacco; $54^{\text {th }}$ Tobacco Science Research Conference, Program Booklet and Abstracts, Vol. 54, Paper No. 45, 2000, pp. 44-45; CORESTA Congress, Lisbon, Portugal, CORESTA Inf. Bull., 2000 Spec. Edition, 2000, Paper ST23, p. 168.

265. Philip Morris USA: Philip Morris USA asks court to declare patent asserted by Star Scientific invalid; www.philipmorrisusa.com/pressroom/content/press_r elease/articles/pr_june_28_2002_pmuactdpabssi.asp.

266. Star Scientific, Inc.: Federal court dismisses Philip Morris lawsuit that challenged Star Scientific patent for reducing cancer-causing toxins; www.starscientific.com/frame_pages/release_frame .htm.

267. Felton K.S. and M.G. Knize: Heterocyclic amine mutagens/carcinogens in foods; in: Chemical mutagenesis and carcinogenesis, edited by C.S. Cooper and P.L. Grover, Springer-Verlag, Berlin/Heidelberg, 1990, pp. 471-502.

268. Clapp, W.L., B.S. Fagg, and C.J. Smith: Reduction in Ames Salmonella mutagenicity of mainstream cigarette smoke condensate by tobacco protein removal; Mutat. Res. 446 (1999) 167-174.

269. Wakeham, H.: Recent trends in tobacco and tobacco smoke research; Am. Chem. Soc. Symposium, 1971; Recent trends in tobacco and tobacco smoke research; in: The chemistry of tobacco and tobacco smoke, edited by I. Schmeltz,. Plenum Press, New York, NY, 1972, pp. 1-20.

270. Wynder, E.L.: Some concepts of the less harmful cigarette; in: A safe cigarette? Banbury Report 3, 
edited by G.B. Gori and F.G. Bock, Cold Spring Harbor Laboratory, Cold Spring Harbor, NY, 1980, pp. 3-12.

271. Gori, G.B.: Less hazardous cigarettes; in: Prevention and detection of cancer. Part 1. Prevention. Vol. 1. Etiology, edited by H.E. Nieburgs, Marcel Dekker, Inc., New York, NY, 1977, pp. 791-804.

272. Gori, G.B.: Low-risk cigarettes: A prescription; Science 194 (1976) 1243-1256; Gori, G.B. and C.J. Lynch: Toward less hazardous cigarettes: Current advances; J. Am. Med. Assoc. 240 (1978) 1255-1259.

273. Aristotle: Nicomachean ethics, Book X; 350 BC, Jowett translation.

274. Rowland, S.: On tobacco; epigram written about 1600.

275. Cook, J.W.: Chemical carcinogens and their significance; Lancet (1957) i, 333-335.

276. Coultson, F.: Overall view of the conference; in: Human epidemiology and animal correlations in chemical carcinogenesis, edited by F. Coultson and P. Shubik, Ablex Publishing Corporation, Norwood, NJ, 1980, pp. 395-402.

277. Peto, R. and R. Doll: The control of lung cancer; New Scientist 105 (1985) 26-30.
278. Occupational Safety and Health Administration: Indoor air quality; Fed. Reg. 59 (No. 65) (1994) 15968-16039, see 15987, Table III-6.

279. Schmeltz, I. and D. Hoffmann: Nitrogen-containing compounds in tobacco and tobacco smoke; Chem. Rev. 77 (1977) 295-311.

280. Cook, J.W., C.L. Hewett, and I. Hieger: Coal-tar constituents and cancer; Nature 130 (1932) 926; Isolation of a cancer-producing hydrocarbons from coal tar. II. Isolation of 1,2- and 4,5-benzopyrenes, perylene, and 1,2-benzanthracene; J. Chem. Soc. (1933) 395-398; Barry, G., J.W. Cook, G.A.D. Haslewood, C.L. Hewett, I. Hieger, and E.L. Kennaway: The production of cancer by pure hydrocarbons. Part III; Proc. Royal Soc. (Biol.) 117 (1935) 318-351.

\section{Address for correspondence}

Alan Rodgman

2828 Birchwood Drive

Winston-Salem, North Carolina, 27103-3410, USA 
In the body of our report we have discussed in considerable detail the many lists of MSS toxicants, most of which were issued over the past few decades. Obviously, various investigators, institutions and government agencies have been extremely zealous in their generation of such lists. We also pointed out the fact that most of the compilers of the lists persist in including MSS components that are no longer relevant since their precursors have not been present in the cigarette filler for decades, components whose presence in MSS is highly suspect, and components for which no or extremely few quantitative analytical data exist. In addition, the compilers persist in listing ranges for cigarette yields of many toxicants and the ranges include analytical data, whether acceptable or poor, generated on cigarettes manufactured in the 1950 s and 1960s. Such data are totally irrelevant to cigarettes manufactured during the past two decades.

Between the mid-1950s and late 1970s extensive research was conducted both within the Tobacco Industry and outside it to define the components in tobacco and its smoke and the relationship between them. Much of the early work revolved around the presence of PAHs, particularly $\mathrm{B} a \mathrm{P}$, in cigarette MSS. Eventually, interest in the PAHs (and $\mathrm{B} a \mathrm{P}$ ) declined and was replaced with interest in NNAs. This interest became even more intense with the discovery in tobacco and smoke of TSNAs, especially NNK. Except for efforts to control the levels of TSNAs in tobacco and smoke, much of the research to define the composition of tobacco smoke declined within the Tobacco Industry and outside it. In our main report, we pointed out some of the problems with the current emphasis on TSNAs and NNK.

While the number of MSS components listed as toxicants has grown steadily, particularly since the IARC 1986 report on smoking (1), numerous other MSS components classified in the literature as toxicants have been omitted from the MSS toxicant lists with no specific reason offered for their omission. In the main part of this report we have written at some length on the omission of the dioxins from all but one list of cigarette MSS toxicants. Even though the precise MSS levels of other omitted toxicants have not been quantified probably because they are extremely low, most of them have been reported as tumorigenic to laboratory animals. Lack of knowledge of per cigarette MSS levels is no excuse for omission. In the lists of tobacco smoke components first categorized as "IARC Group 2A carcinogens" or "IARC Group 2B carcinogens", the levels of several were originally recorded in the "IARC Group 2B" category only as "present" or "present in trace amounts" (2). These included benzo[b]furan, caffeic acid, dibenzo[a,e]pyrene $\{$ naphtho[ $[1,2,3,4-$ $d e f]$ chrysene $\}$, and the much discussed PAH dibenzo $[a, l]$ pyrene \{dibenzo[def,p]pyrene $\}$.

The constituent first reported in the late $1950 \mathrm{~s}$ as the $\mathrm{C}_{24} \mathrm{H}_{14} \mathrm{PAH}$ 1,2,3,4-dibenzopyrene, later named dibenzo[ $a, l]$ pyrene and then dibenzo[def,p]pyrene (I), (3-7) was subsequently shown in 1966 to be the isomeric dibenz $[a, e]$ aceanthrylene (II) (also known as<smiles></smiles>

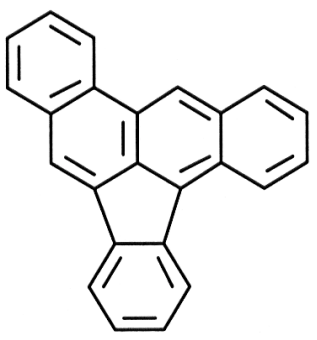

I II

Appendix Figure 1. Dibenzo[a,Ipyrene \{dibenzo[def,p]chrysene (I), dibenz[a,e]aceanthrylene \{dibenzo[a,e]fluoranthene\} (II) dibenzo[a,e]fluoranthene) (8) (see Appendix Figure 1). The authentic dibenzo $[a, l]$ pyrene was subsequently identified in MSS (9).

We find the dibenzo $[a, l]$ pyrene-dibenzo $[a, e]$ aceanthrylene situation to be an interesting one. Several investigators reported dibenzo[ $a, l]$ pyrene to be present in MSS, others reported MSS yields from various late 1950 s cigarettes, e.g., $16 \mathrm{ng} / \mathrm{cig}$ by LYONS (4), $0.02 \mathrm{ng} / \mathrm{cig}$ by VAN DUUREN (5), $0.6 \mathrm{ng} / \mathrm{cig}$ by RODGMAN and COOK (6). When the identity error was resolved by LAVIT-LAMY and BUU-HOÏ (8) in 1966 and acknowledged by HOFFMAN and WYNDER (10) and others involved in tobacco smoke composition studies, some agencies, particularly IARC (1), persisted in listing dibenzo[a,l]pyrene as present in MSS, using the 1958 VAN DUUREN report (5) as authority. The yield data, such as they are from the late $1950 \mathrm{~s}$, were for dibenz[a,e]aceanthrylene not for dibenzo[ $[a, l]$ pyrene. The per cigarette yield ranged from 0.02 to $16 \mathrm{ng} / \mathrm{cig}$. Despite the range and the knowledge that the MSS component dibenz[a,e]aceanthrylene (II) is tumorigenic to mouse skin and a known initiator (11), it is never listed as an MSS toxicant. Dibenzo[ $a, l]$ pyrene with similar biological properties, no quantitative yield data, and only described as present is repeatedly listed as a significant tumorigen in MSS. As we noted in our main report, the paucity of data on dibenzo[ $[a, l]$ pyrene vs. the wealth of data on $\mathrm{B} a \mathrm{P}$ raises serious questions as to why they both are considered equally significant tumorigens in cigarette MSS.

While benzo[ $a, l]$ pyrene has been included in toxicant list after list, HECHT (12) commented that its presence in cigarette smoke has not been confirmed. We feel that one has to weigh HECHT's comment against the current status of defined MSS composition. Since the appreciable decline in detailed MSS composition studies after the late 1970s, no one or no group has attempted to confirm the identities of the great number of PAHs $(9,13,14)$, azaarenes $(15,16)$, nitrogen-containing components $(19)$, or ether(18) and water-soluble components (19) newly reported in cigarette MSS in the 1970s. Examination of the post-1980 literature indicates that the identities of nearly half the components described in those studies have never been confirmed. Because of that, would HECHT also discount their presence in MSS in the same way he discounts the presence of benzo[ $[a, l]$ pyrene?

In some ways the inclusion or exclusion of a specific toxicant from a list appears to be somewhat of a "copy cat" syndrome. For example, the 1986 HOFFMANN-HECHT list (20) includes chrysene but not $N$-nitrosodi- $n$-propylamine or $N$-nitrosodi- $n$-butylamine. The 1994 OSHA (21) list omits chrysene but includes the two NNAs. In their 1997 list (22), HoFFMANN and HOFFMANN include the two NNAs but not chrysene.

In Appendix Table 1 we have listed over two dozen other MSS components demonstrated to exhibit adverse biological properties in one or more species of laboratory animals that are seldom, if ever, included in lists of MSS toxicants.

From the mid-1950s to the late 1970s, the number of completely or partially identified PAHs in cigarette MSS increased from very few in 1954 (54) to over 90 by 1964 (55) to more than 500 reported from the seminal study by SNOOK et al., USDA personnel at Athens, GA $(9,13,14)$. A somewhat similar chronological situation existed with the number of identified aza-arenes in MSS from the mid-1950s to 1981 when the list of identified aza-arenes was expanded by HECKMAN and BEST (17) and again by the USDA, Athens, GA personnel (15). However, the situation is completely different with the NNAs.

During extensive investigations of the composition of tobacco smoke in general and cigarette MSS in particular, much effort was expended in the early 1960s to define the nature of $N$-nitrosation during curing and the smoking process. As more and more NNAs were identified in tobacco and/or tobacco smoke, they were categorized as follows: Volatile NNAs, nonvolatile NNAs, 
Appendix Table 1. Cigarette mainstream smoke components with reported biological activity, including some with tumorigenic properties

\begin{tabular}{|c|c|c|c|}
\hline Tobacco smoke component & CAS no. & References & Biological activity \\
\hline \multicolumn{4}{|c|}{ Polycyclic aromatic hydrocarbons } \\
\hline Anthracene, 9,10-dimethyl- & $781-43-1$ & Rothwell and Whitehead (23) & Dipple et al. (24) ${ }^{\text {a }}$ \\
\hline Benz[a]anthracene, 7,12-dimethyl- & $57-97-8$ & Pietzsch (25) & Hartwell (26) ${ }^{\mathrm{b}}$; Shubik and Hartwell (27) ${ }^{\mathrm{b}}$ \\
\hline Benz[a]anthracene, ethyl- & $31632-62-9$ & Lee et al. (28) & $\begin{array}{l}\text { Dipple et al. (24) } \\
\quad \text { at least two of the ethylbenz[a]anthracenes } \\
\text { are tumorigenic to mouse skin\} }\end{array}$ \\
\hline Benz[a]anthracene, 5-methyl- & $2319-96-2$ & Bonnet and Neukomm (29) & Dipple et al. (24) \\
\hline Benz[a]anthracene, 6-methyl- & $316-14-3$ & Lee et al. (28) & Dipple et al. (24) \\
\hline Benz[a]anthracene, 8-methyl- & 2381-31-9 & Lee et al. (28) & Dipple et al. (24) \\
\hline Benz[a]anthracene, trimethyl- & $60826-78-0$ & Lee et al. (28); Snook et al. $(13,14)$ & $\begin{array}{l}\text { Dipple et al. }(24) \\
\quad \text { sseveral trimethylbenz[a]anthracenes are } \\
\text { tumorigenic to mouse skin\} }\end{array}$ \\
\hline Benzo[c]phenanthrene & $195-19-7$ & Van Duuren (5); Snook et al. (9) & Dipple et al. (24) \\
\hline Benzo[c]phenanthrene, methyl- & & $\begin{array}{l}\text { Brunnemann and Hoffmann (30); Van } \\
\text { Duuren (5) }\end{array}$ & $\begin{array}{l}\text { Dipple et al. }(24) \\
\text { \{several methylbenzo[c]phenanthrenes are } \\
\text { tumorigenic to mouse skin\} }\end{array}$ \\
\hline Benzo[b]triphenylene & $215-58-7$ & Snook et al. (9) & Dipple et al. (24) \\
\hline Dibenz $[a, e]$ aceanthrylene & $5385-75-1$ & $\begin{array}{l}\text { Wynder and Wright (3); Lyons (4); Van } \\
\text { Duuren (5); Rodgman and Cook (6); } \\
\text { Pyriki (7) }\end{array}$ & IARC (11) \\
\hline Dibenz[a,j]anthracene & $224-41-9$ & Snook et al. (9) & Dipple et al. (24) \\
\hline $13 H$-Dibenzo[a, $\mathrm{l}$ fluorene & $239-60-1$ & Lyons and Johnston (31); Pyriki (7) & Hartwell (26) \\
\hline Phenanthrene, 1,2,3,4-tetramethyl- & $71607-70-0$ & Snook et al. (13) & Hartwell (26) \\
\hline \multicolumn{4}{|c|}{ Aza-arenes } \\
\hline Benz[a]acridine & $225-11-6$ & $\begin{array}{l}\text { Rothwell and Whitehead }(23,32) \text {; } \\
\text { Grimmer et al. (33) }\end{array}$ & IARC (1) \\
\hline Benz $[c]$ acridine & $225-51-4$ & $\begin{array}{l}\text { Rothwell and Whitehead }(23,32) \text {; } \\
\text { Snook et al. (15) }\end{array}$ & IARC (1) \\
\hline Benz[c]acridine, 7,9-dimethyl- & 963-89-3 & Klimisch and Beiss (34) & Dipple et al. (24) \\
\hline Benz $[c]$ acridine, 7,10-dimethyl- & $2381-40-0$ & Klimisch and Beiss (34) & Dipple et al. (24) \\
\hline Benz[c]acridine, 7-methyl- & $3340-94-1$ & Grimmer et al. (33) & Dipple et al. (24) \\
\hline \multicolumn{4}{|c|}{ Amines } \\
\hline Aniline, 2-methoxy- & $90-04-0$ & Pailer et al. (35) & IARC (1) \\
\hline \multicolumn{4}{|c|}{ Phenols } \\
\hline Catechol, 3-methyl- & $488-17-5$ & Brunnemann et al. (36) & $\operatorname{IARC}(37)^{c}$ \\
\hline Catechol, 4-methyl- & $452-86-5$ & Brunnemann et al. (36) & IARC (1) \\
\hline Eugenol & $97-53-0$ & Rodgman and Cook (38) & NTIS (39) \\
\hline Isoeugenol & $97-54-1$ & Rodgman and Cook (38) & NTIS (40) \\
\hline \multicolumn{4}{|c|}{ Quinones } \\
\hline 1,4-Benzoquinone & $106-51-4$ & $\begin{array}{l}\text { Bonnet and Neukomm (29); } \\
\text { Schmeltz et al. (41) }\end{array}$ & $\begin{array}{l}\text { Takizawa (42) } \\
\text { Tiedemann (43) }\end{array}$ \\
\hline 1,2-Naphthoquinone & $542-42-5$ & Benner et al. (44) & Takizawa (42) \\
\hline 1,4-Naphthoquinone & $130-15-4$ & Schmeltz et al. (41); Snook et al. (45) & Takizawa (42) \\
\hline \multicolumn{4}{|c|}{ Carbohydrates } \\
\hline Fructose & $57-48-7$ & Kobashi and Sakaguchi (46) & Takizawa (47) \\
\hline Glucose & $26655-34-5$ & Kobashi and Sakaguchi (46) & Takizawa $(47,48)$ \\
\hline \multicolumn{4}{|c|}{ Miscellaneous compounds } \\
\hline Chloroform & $67-66-3$ & Holzer et al. (49) & IARC (50) \\
\hline Coumarin & $91-64-5$ & Grob and Völlmin (51) & IARC $(37,52)$ \\
\hline Maleic anhydride $\{2,5$-furandione $\}$ & $108-31-6$ & Schumacher et al. (19) & IARC (37) \\
\hline Maleic anhydride, 2,3-dimethyl- & $766-39-2$ & Schumacher et al. (19) & IARC 37) \\
\hline Succinic anhydride & $108-30-5$ & Schumacher et al. (19) & IARC $(37,53)$ \\
\hline
\end{tabular}

a Dipple et al. (24) have a tabulation of the tumorigenicity to mouse skin of a wide variety of PAHs and aza-arenes.

${ }^{b}$ Hartwell (26) and Shubik and Hartwell (27) have numerous references to the tumorigenicity of this PAH.

${ }^{c}$ IARC (37) lists 3-methylcatechol, 4-methylcatechol, maleic anhydride, 2,3-dimethylmaleic anhydride, succinic anhydride, and coumarin as biologically activity components of cigarette MSS.

TSNAs, and $N$-nitrosamino acids. Within these four categories, only about 40 NNAs have been identified to date as tobacco and/or tobacco smoke components. Except for an excursion into the identification of $N$-nitrosamino acids, identification of NNAs in MSS almost ceased when NNK and to some extent NNN became the toxicants of choice. This situation raises the question: If a detailed study similar to those conducted on the PAHs and aza-arenes were conducted, how many additional NNAs could be identified in tobacco and/or tobacco smoke?

Appendix Table 2 lists several NNAs reported as tobacco components that are seldom discussed. To date, none of them has been identified in tobacco smoke.
Appendix Table 3 lists the NNAs from which those usually classified as toxicants are selected. Of the NNAs in MSS defined as volatile NNAs, 11 are $N$-nitrosodialkylamines.

In Appendix Table 4 are listed 22 dialkylamines, identified in tobacco and/or smoke as the amine or the NNA. While Appendix Table 4 is not necessarily complete, it suffices for the following discussion: For four NNAs ( $N$-nitrosoisobutylmethylamine, $N$ nitrosoethylpropylamine, $N$-nitrosoethylisobutylamine, $N$-nitroso$n$-butylethylamine), the corresponding amines have not been identified in tobacco smoke. It is highly probable that the four amines are present as MSS components. Alternatively, NNAs corresponding to the other ten dialkylamines identified in tobacco 


\begin{tabular}{|c|c|c|c|c|}
\hline \multirow[b]{2}{*}{$N$-Nitrosamine } & \multirow[b]{2}{*}{ CAS no. } & \multicolumn{2}{|c|}{ Identified in tobacco $(\mathrm{T})$ and/or smoke $(\mathrm{S})$} & \multirow[b]{2}{*}{ Activity } \\
\hline & & $\mathrm{T}$ & S & \\
\hline 1-Nitroso-2-azetidinecarboxylic acid & $55556-98-4$ & $\mathrm{x}$ & - & \\
\hline 4-(N-Methylnitrosamino)-1-(3-pyridinyl)butanone oxide & $76014-82-9$ & $x$ & - & $(+)^{\mathrm{a}}$ \\
\hline 1-Nitroso-4-hydroxyproline & 2443-30-3 & $x$ & - & \\
\hline 1-Nitroso-3-piperidinecarboxylic acid & $65445-62-7$ & $\mathrm{x}$ & - & \\
\hline 1-Nitroso-4-piperidinecarboxylic acid & $6238-69-3$ & $x$ & - & $(-)[173]^{b}$ \\
\hline 3-Nitroso-4-thiazolidinecarboxylic acid & $88381-44-6$ & $x$ & - & \\
\hline
\end{tabular}

${ }^{a}$ Bioassay results reported by Castonguay et al. (56).

${ }^{b}$ Bioassay results in laboratory animals are summarized in Preussmann and Stewart (57). (+) indicates tumor induction, (-) indicates negative response. Number in [ ] represents catalog number in Preussmann and Stewart (57).

Appendix Table 3. $\mathrm{N}$-Nitrosamines in tobacco and/or tobacco smoke

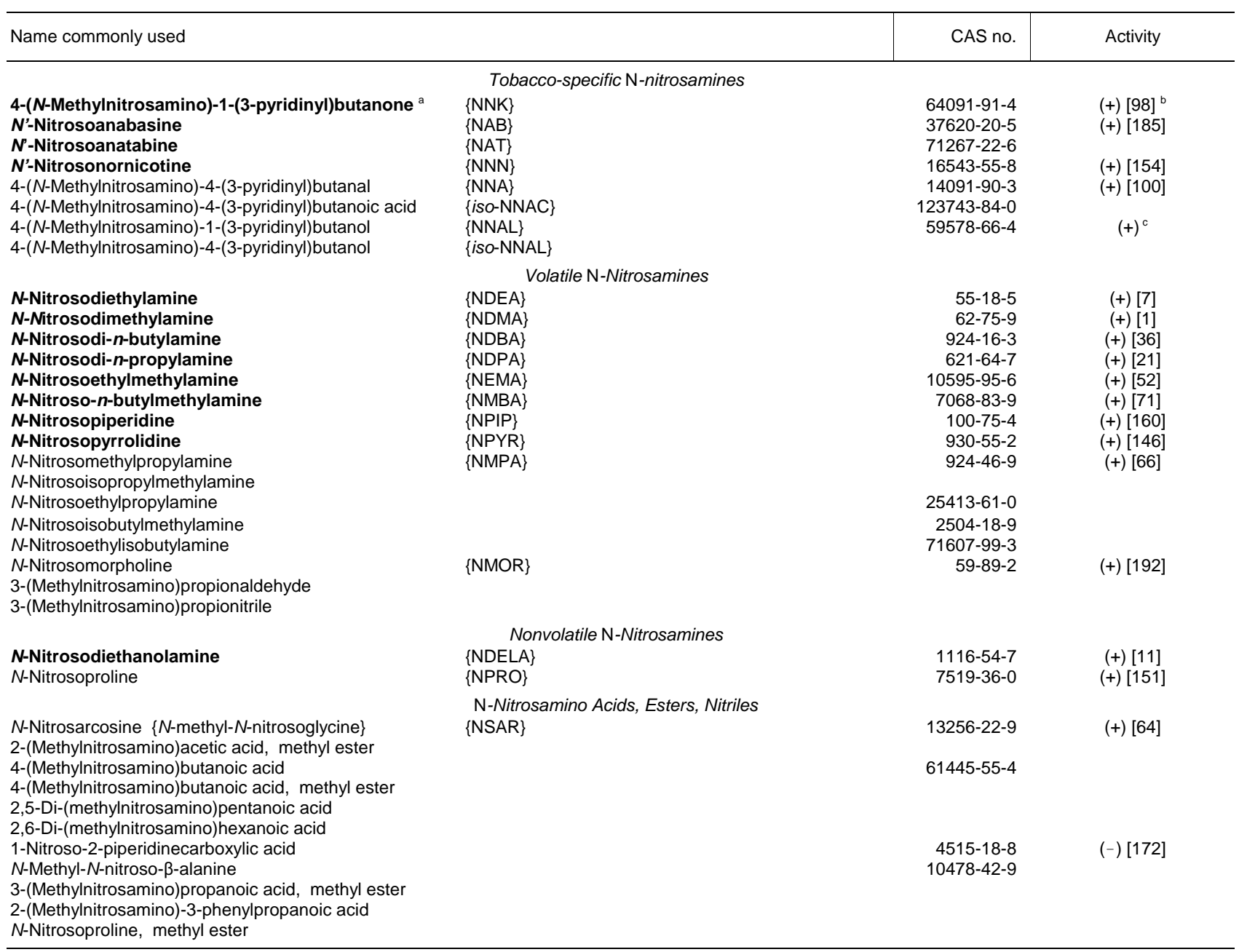

${ }^{a}$ Compounds listed in bold are included in Table 1 of our main report

${ }^{\mathrm{b}}(+)$ indicates tumor induction; (-) indicates no response. Number in [ ] represents catalog number in Preussmann and Stewart (57).

' See Castonguay et al. (56).

and/or tobacco smoke have not yet been identified in smoke, e.g., no NNA corresponding to sec-butylmethylamine, isopentylmethylamine, or isopropylidenemethylamine identified as MSS components has been identified in MSS. Synthetically, the corresponding NNAs are as easily prepared as $N$-nitrosodimethylamine or $N$-nitrosodiethylamine so their pyrogenesis during the smoking process should not be hindered. Thus, it is highly probable that the ten NNAs are present in tobacco smoke. For each of the six NNAs listed as toxicants in Table 1 of the main body of our report, the corresponding amine has been identified in tobacco and/or tobacco smoke.
Many other secondary amines have been identified in tobacco smoke but for most of them no corresponding NNA has been identified in smoke. These include a series of $N$-substituted anilines, all amenable to $N$-nitrosation. Others include the alkyl derivatives of pyrrolidine $(\mathbf{I V})$, piperazine $(\mathbf{V})$, and piperidine $(\mathbf{V I})$ (Appendix Figure 2). The amines pyrrolidine (IV), piperidine (VI), and 1,2,3,6-tetrahydropyridine (VII) have been identified in cigarette MSS but not piperazine (V).

For each of the piperazines, mono- and di- $N$-nitroso derivatives are possible. In many instances, the NNAs are readily synthesized and have been tested for tumorigenicity [see PREUSSMANN and STEWARD (57)]. 


\begin{tabular}{|c|c|c|c|c|c|c|c|c|c|}
\hline \multicolumn{2}{|c|}{$\mathrm{R}_{1}-\mathrm{NH}-\mathrm{R}_{2}$} & \multirow[b]{2}{*}{ CAS no. } & \multicolumn{2}{|c|}{$\begin{array}{c}\text { Identified in tobacco } \\
(\mathrm{T}) \text { or smoke (S) }\end{array}$} & \multirow{2}{*}{ N-Nitrosamine } & \multirow[b]{2}{*}{ CAS no. } & \multicolumn{2}{|c|}{\begin{tabular}{|c|} 
Identified in tobacco \\
$(\mathrm{T})$ or smoke (S)
\end{tabular}} & \multirow[b]{2}{*}{ Activity } \\
\hline$R_{1}=$ & $R_{2}=$ & & $\mathrm{T}$ & $\mathrm{S}$ & & & $\mathrm{T}$ & $\mathrm{S}$ & \\
\hline $\mathrm{CH}_{3}^{-}$ & $\mathrm{CH}_{3}^{-}$ & $124-40-3$ & $\mathrm{x}$ & $\mathrm{x}$ & N-Nitrosodimethylamine ${ }^{a}$ & $62-75-9$ & $\mathrm{x}$ & $\mathrm{x}$ & $(+)[1]^{b}$ \\
\hline $\mathrm{CH}_{3}-$ & $\mathrm{CH}_{3} \mathrm{CH}_{2}^{-}$ & $624-78-2$ & $x$ & $x$ & N-Nitrosoethylmethylamine & $10595-95-6$ & $x$ & $x$ & $(+)[52]^{b}$ \\
\hline $\mathrm{CH}_{3}-$ & $\mathrm{CH}_{3}\left(\mathrm{CH}_{2}\right)_{2-}^{-}$ & $627-35-0$ & $x$ & - & N-Nitrosomethylpropylamine & $924-46-9$ & - & $x$ & $(+)[66]^{\mathrm{b}}$ \\
\hline $\mathrm{CH}_{3}^{-}$ & $\left(\mathrm{CH}_{3}\right)_{2} \mathrm{CH}-$ & $4747-21-1$ & $x$ & $x$ & N-Nitrosoisopropylmethylamine & $34419-76-6$ & $\mathrm{x}$ & $x$ & \\
\hline $\mathrm{CH}_{3}-$ & $\mathrm{CH}_{3}\left(\mathrm{CH}_{2}\right)_{3}^{-}$ & $110-68-9$ & $x$ & $x$ & N-Nitrosobutylmethylamine & 7068-83-9 & - & $x$ & $(+)[71]^{b}$ \\
\hline $\mathrm{CH}_{3}-$ & $\left(\mathrm{CH}_{3}\right)_{2} \mathrm{CHCH}_{2^{-}}$ & $2504-18-9$ & - & - & N-Nitrosoisobutylmethylamine & & - & $x$ & \\
\hline $\mathrm{CH}_{3}^{-}$ & $\left(\mathrm{CH}_{3}\right)\left(\mathrm{C}_{2} \mathrm{H}_{5}\right) \mathrm{CH}-$ & & - & $\mathrm{x}$ & $\mathrm{N}$-Nitroso-sec-butylmethylamine & & - & - & \\
\hline $\mathrm{CH}_{3}-$ & $\left(\mathrm{CH}_{3}\right)_{2} \mathrm{CH}\left(\mathrm{CH}_{2}\right)_{2}-$ & & - & $x$ & $\mathrm{~N}$-Nitrosoisopentylmethylamine & & - & - & \\
\hline $\mathrm{CH}_{3-}^{-}$ & $\mathrm{CH}_{2}=\mathrm{C}\left(\mathrm{CH}_{3}\right)-$ & 22023-64-9 & - & $x$ & $\mathrm{~N}$-Nitrosoisopropylidenemethylamine & & - & - & \\
\hline $\mathrm{CH}_{2} \mathrm{CH}_{3}-$ & $\mathrm{CH}_{2} \mathrm{CH}_{3}-$ & $109-89-7$ & $x$ & $x$ & $\mathrm{~N}$-Nitrosodiethylamine & $55-18-5$ & - & $x$ & $(+)[7]^{\mathrm{b}}$ \\
\hline $\mathrm{CH}_{2} \mathrm{CH}_{3}-$ & $\mathrm{CH}_{3}\left(\mathrm{CH}_{2}\right)_{2}^{-}$ & 20193-20-8 & - & - & $\mathrm{N}$-Nitrosoethylpropylamine & $25413-61-0$ & - & $\hat{x}$ & \\
\hline $\mathrm{CH}_{2} \mathrm{CH}_{3}-$ & $\left(\mathrm{CH}_{3}\right)_{2} \mathrm{CHCH}_{2}-$ & & - & - & N-Nitrosoethylisobutylamine & $71607-99-3$ & - & $x$ & \\
\hline $\mathrm{CH}_{2} \mathrm{CH}_{3}^{-}$ & $\mathrm{CH}_{3}\left(\mathrm{CH}_{2}\right)_{3}^{-}$ & $13360-63-9$ & - & - & $N$-Nitroso- $n$-butylethylamine & & - & $\hat{x}$ & $(+)[122]^{b}$ \\
\hline $\mathrm{CH}_{3}^{-}\left(\mathrm{CH}_{2}\right)_{2}^{-}$ & $\mathrm{CH}_{3}\left(\mathrm{CH}_{2}\right)_{2^{-}}^{-}$ & $142-84-7$ & $x$ & $x$ & $N$-Nitrosodi- $n$-propylamine & $621-64-7$ & - & $\mathrm{x}$ & $(+)[21]^{b}$ \\
\hline $\mathrm{CH}_{3}\left(\mathrm{CH}_{2}\right)_{2}^{-}$ & $\left(\mathrm{CH}_{3}\right)_{2} \mathrm{CH}-$ & $21968-17-2$ & $x$ & $x$ & N-Nitrosoisopropylpropylamine & & - & - & \\
\hline $\mathrm{CH}_{3}\left(\mathrm{CH}_{2}\right)_{2^{-}}$ & $\left(\mathrm{CH}_{3}\right)\left(\mathrm{C}_{2} \mathrm{H}_{5}\right) \mathrm{CH}-$ & & - & $x$ & $\mathrm{~N}$-Nitroso-sec-butylpropylamine & & - & - & \\
\hline$\left(\mathrm{CH}_{3}\right)_{2} \mathrm{CH}-$ & $\left(\mathrm{CH}_{3}\right)_{2} \mathrm{CH}-$ & 108-18-9 & - & $x$ & $\mathrm{~N}$-Nitrosodiisopropylamine & $601-77-4$ & - & - & $(+)[34]^{b}$ \\
\hline$\left(\mathrm{CH}_{3}\right)_{2} \mathrm{CH}-$ & $\mathrm{CH}_{3}\left(\mathrm{CH}_{2}\right)_{3}^{-}$ & $39099-23-5$ & - & $x$ & $\mathrm{~N}$-Nitrosobutylisopropylamine & & - & - & \\
\hline $\mathrm{CH}_{3}\left(\mathrm{CH}_{2}\right)_{3}^{-}$ & $\mathrm{CH}_{3}\left(\mathrm{CH}_{2}\right)_{3}^{-}$ & $111-92-2$ & $x$ & - & $N$-Nitrosodi- $n$-butylamine & $924-16-3$ & $x$ & $x$ & $(+)[36]^{b}$ \\
\hline $\mathrm{CH}_{3}\left(\mathrm{CH}_{2}\right)_{3}^{-}$ & $\left(\mathrm{CH}_{3}\right)_{2} \mathrm{CHCH}_{2}^{-}$ & $20810-06-4$ & - & $x$ & $\mathrm{~N}$-Nitrosobutylisobutylamine & & - & - & \\
\hline$\left(\mathrm{CH}_{3}\right)\left(\mathrm{C}_{2} \mathrm{H}_{5}\right) \mathrm{CH}$ & - $\left(\mathrm{CH}_{3}\right)\left(\mathrm{C}_{2} \mathrm{H}_{5}\right) \mathrm{CH}-$ & 626-23-3 & $x$ & - & $N$-Nitrosodi-sec-butylamine & & - & - & $(+)[45]^{b}$ \\
\hline$\left(\mathrm{CH}_{3}\right)_{3} \mathrm{C}-$ & $\left(\mathrm{CH}_{3}\right)_{2} \mathrm{CH}-$ & & - & $x$ & $\mathrm{~N}$-Nitroso-tert-butylisopropylamine & & - & - & \\
\hline
\end{tabular}

${ }^{a}$ Compounds displayed in bold are listed as toxicants in Table 1 of the main part of our report.

${ }^{b}$ Bioassay results in laboratory animals are summarized in Preussmann and Stewart (57). (+) indicates tumor induction, (-) indicates negative response. Number in [ ] is catalog number in Preussmann and Stewart (57).<smiles>[R]NC1CCCN1</smiles><smiles>C1CNCCN1</smiles>

V

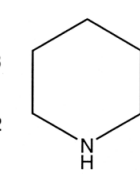

VI

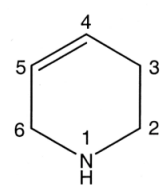

VII
Appendix Figure 2. An aromatic amine (III), pyrrolidine (IV), piperazine (V), piperidine (VI), 1,2,3,6-tetrahydropyridine (VII)<smiles>[X]c1ccccc1C1C=CC=CC12Nc1ccccc12</smiles>

Appendix Figure 3. Indole (VIII), carbazole (IX), and $\mathbf{H}$-benzimidazole (X)

In Appendix Table 5 are listed 32 secondary amines, most of which have been identified in tobacco and/or smoke. In only a few cases have the corresponding NNAs been identified as tobacco and/or smoke components. It is highly probable that the NNAs corresponding to the remaining secondary amines are also tobacco smoke components.

Among the numerous classes of smoke components are several other types of secondary amines, e.g., the pyrroles, indoles, carbazoles, imidazoles. However, their highly aromatic nature and the acidity of the imino hydrogen probably preclude any significant $N$-nitrosation either in the tobacco or during the smoking process. A dozen or so substituted pyrroles; nearly 50 alkyl derivatives of indole (VIII); carbazole (IX) and several of its alkyl derivatives, benzocarbazoles, and dibenzocarbazoles; and several alkyl derivatives of imidazole and benzimidazole $(\mathbf{X})$ have been reported as tobacco smoke components. Even though each of them could theoretically yield an NNA, no NNA corresponding to any of them has been identified to date in tobacco smoke (Appendix Figure 3).

It is obvious that the number of NNAs in tobacco/tobacco smoke might be substantially greater than the 40 or so NNAs now known to be present. Since the per cigarette yields of the yet unidentified NNAs may be at the picogram or femtogram levels, their contribution to MSS toxicological properties may not be particularly meaningful or important. However, they may be just as important from a biological point of view as those MSS components repeatedly listed as toxicants for which no or questionable quantitative data are available.

To put the NNAs in perspective and to determine how many more are actually present in MSS, what may be needed is an extensive study corresponding to the PAH $(9,13,14)$ and aza-arene (15) studies conducted in the 1970s.

It is also interesting to note that 4-(methylnitrosamino)-1-(3-pyridyl)-1-butanol (NNAL), the major metabolite of NNK (12), is usually not listed as a cigarette MSS toxicant even though NNAL has been reported to be both tumorigenic to several rodent species (56) and mutagenic in the Ames Salmonella typhimurium test. While the MSS toxicants listed in Table 1 in the main body of our report number about 150 , their number could be increased in future published lists (58) by inclusion of the individual dioxins plus components in Appendix Tables 1, 4, and 5. The increase in number would far outweigh the decrease resulting from deletion of the problematic components we discussed earlier.

\section{REFERENCES}

1. IARC: Chemistry and analysis of tobacco smoke; in: Evaluation of the carcinogenic risk of chemicals to humans: Tobacco smoking; IARC, Lyon, France, IARC Monograph 38 (1986) 83-126, 387-394.

2. Smith, C.J., T.A. Perfetti, M.A. Rumple, A. Rodgman, and D.J. Doolittle: "IARC Group 2B carcinogens" reported in cigarette mainstream smoke; Food Chem. Toxicol. 39 (2001) 183-205. 


\begin{tabular}{|c|c|c|c|c|c|c|c|c|c|}
\hline \multirow{2}{*}{\multicolumn{2}{|c|}{ Amine }} & \multirow[b]{2}{*}{ CAS no. } & \multicolumn{2}{|c|}{$\begin{array}{c}\text { Identified in tobacco } \\
(\mathrm{T}) \text { or smoke }(\mathrm{S}) \\
\end{array}$} & \multirow{2}{*}{$N$-Nitrosamine } & \multirow[b]{2}{*}{ CAS no. } & \multicolumn{2}{|c|}{$\begin{array}{l}\text { Identified in tobacco } \\
\text { (T) or smoke (S) }\end{array}$} & \multirow[b]{2}{*}{ Activity } \\
\hline & & & $\mathrm{T}$ & $\mathrm{S}$ & & & $\mathrm{T}$ & S & \\
\hline \multicolumn{10}{|c|}{ Aromatic amine (III) $R_{1}-\mathrm{NH}-R_{2}$} \\
\hline $\mathrm{R}_{1}=$ & $\mathrm{R}_{2}=$ & & & & & & & & \\
\hline $\mathrm{CH}_{3}^{-}$ & $\mathrm{C}_{6} \mathrm{H}_{5}-$ & $100-61-8$ & - & $x$ & $N$-Nitroso- $N$-methylphenylamine & $614-00-6$ & - & - & $(+)[108]^{a}$ \\
\hline $\mathrm{CH}_{3}-$ & $2-\mathrm{CH}_{3}-\mathrm{C}_{6} \mathrm{H}_{4}-$ & $611-21-2$ & - & $x$ & $\mathrm{~N}$-Nitroso- $\mathrm{N}$-methyl-2-toluidine & & - & - & \\
\hline $\mathrm{CH}_{3}-$ & $4-\mathrm{CH}_{3}-\mathrm{C}_{6} \mathrm{H}_{4}^{-}$ & $623-08-5$ & - & $x$ & $N$-Nitroso- $N$-methyl-4-toluidine & & - & - & \\
\hline $\mathrm{CH}_{3}^{-}$ & $2-\mathrm{C}_{2} \mathrm{H}_{5}-\mathrm{C}_{6} \mathrm{H}_{4}^{-}$ & $1821-38-1$ & - & $x$ & $\mathrm{~N}$-Nitroso-2-ethyl- $\mathrm{N}$-methylaniline & & - & - & \\
\hline $\mathrm{CH}_{3}^{-}$ & $3-\mathrm{C}_{2} \mathrm{H}_{5}-\mathrm{C}_{6} \mathrm{H}_{4}^{-}$ & $71265-20-8$ & - & $x$ & $\mathrm{~N}$-Nitroso-3-ethyl- $\mathrm{N}$-methylaniline & & - & - & \\
\hline $\mathrm{CH}_{3}-$ & $4-\mathrm{C}_{2} \mathrm{H}_{5}-\mathrm{C}_{6} \mathrm{H}_{4}-$ & $37846-06-3$ & - & $x$ & $N$-Nitroso-4-ethyl- $N$-methylaniline & & - & - & \\
\hline $\mathrm{CH}_{3}-$ & $\mathrm{C}_{6} \mathrm{H}_{5}-\left(\mathrm{CH}_{2}\right)_{2}-$ & $589-08-2$ & - & $x$ & $N$-Nitroso- $N$-methylphenylethylamine & & - & - & \\
\hline $\mathrm{CH}_{3}-$ & $4-\mathrm{NH}_{2}-\mathrm{C}_{6} \mathrm{H}_{4}^{-}$ & & - & $x$ & $\mathrm{~N}$-Nitroso- $\mathrm{N}$-methyl-4-aminoaniline & & - & - & \\
\hline $\mathrm{C}_{2} \mathrm{H}_{5}$ & $\mathrm{C}_{6} \mathrm{H}_{5}-$ & $103-69-5$ & - & $x$ & $N$-Nitroso- $N$-ethylaniline & $612-64-6$ & - & - & \\
\hline $\mathrm{C}_{2} \mathrm{H}_{5}$ & $2-\mathrm{CH}_{3}-\mathrm{C}_{6} \mathrm{H}_{4}^{-}$ & $94-68-8$ & - & $x$ & $N$-Nitroso- $N$-ethyl-2-toluidine & & - & - & \\
\hline $\mathrm{C}_{6} \mathrm{H}_{5}$ & $\mathrm{C}_{6} \mathrm{H}_{5}-$ & $122-39-5$ & - & $\hat{x}$ & $\mathrm{~N}$-Nitrosodiphenylamine & $86-30-6$ & - & - & $(-)[55]^{a}$ \\
\hline $\mathrm{C}_{6} \mathrm{H}_{5}$ & 4- $\left(\mathrm{CH}_{3}\right)_{2} \mathrm{CH}-\mathrm{C}_{6} \mathrm{H}_{4}^{-}$ & $5650-10-2$ & - & $x$ & 4-Isopropyl- $N$-nitrosodiphenylamine & & - & - & \\
\hline \multicolumn{2}{|c|}{ Pyrrolidine (IV) } & $123-75-1$ & - & $x$ & $N$-nitrosopyrrolidine & $930-55-2$ & - & $x$ & $(+)[146]^{a}$ \\
\hline \multicolumn{2}{|c|}{$2-\mathrm{CH}_{3}-$} & 765-38-8 & $x$ & $x$ & N-Nitroso-2-methylpyrrolidine & & $x$ & - & \\
\hline \multicolumn{2}{|c|}{$3-\mathrm{CH}_{3}^{-}$} & $34375-89-8$ & 一 & $x$ & N-Nitroso-3-methylpyrrolidine & & - & - & \\
\hline \multicolumn{2}{|c|}{ 2,4-diCH $3^{-}$} & $13603-04-8$ & - & $x$ & N-Nitroso-2,4-dimethylpyrrolidine & & - & - & \\
\hline \multicolumn{2}{|c|}{$2,5-\mathrm{diCH}_{3}-$} & $3378-71-0$ & - & $x$ & $\mathrm{~N}$-Nitroso-2,5-dimethylpyrrolidine & $55556-86-0$ & - & - & $(+)[148]^{a}$ \\
\hline \multicolumn{2}{|c|}{$2-\mathrm{CH}_{3} \mathrm{CH}_{2}-$} & $1003-28-7$ & - & $x$ & N-Nitroso-2-ethylpyrrolidine & & - & - & \\
\hline \multicolumn{2}{|c|}{ 2-keto- } & $616-45-5$ & $x$ & - & $\mathrm{N}$-Nitroso-2-pyrrolidone & & - & - & $(-)[301]^{a}$ \\
\hline \multirow{2}{*}{\multicolumn{2}{|c|}{ Piperazine (V) }} & $110-85-0$ & - & - & $N$-Nitrosopiperazine & $5632-47-3$ & - & - & $(+)[200]^{a}$ \\
\hline & & & & & $N, N$ '-Dinitrosopiperazine & 140-79-4 & - & - & $(+)[207]^{a}$ \\
\hline \multicolumn{2}{|c|}{$1-\mathrm{CH}_{3}^{-}$} & $109-01-3$ & - & $x$ & $N$-Nitroso- $N$-methylpiperazine & $16339-07-4$ & - & - & $(+)[201]^{a}$ \\
\hline \multirow{2}{*}{\multicolumn{2}{|c|}{$\begin{array}{l}2-\mathrm{CH}_{3}^{-} \\
2,5-\mathrm{diCH}_{3}-\end{array}$}} & $109-07-9$ & - & $x$ & $N, N$ '-Dinitroso-2-methylpiperazine & $55556-94-0$ & - & - & $(+)[208]^{a}$ \\
\hline & & $106-55-8$ & - & $x$ & $\begin{array}{l}N, N \text {-Dinitroso-2,5- } \\
\text { dimethylpiperazine }\end{array}$ & $55556-88-2$ & - & - & $(+)[209]^{a}$ \\
\hline \multicolumn{2}{|c|}{ Piperidine (VI) } & $110-89-4$ & - & $x$ & N-Nitrosopiperidine ${ }^{b}$ & $100-75-4$ & & $x$ & $(+)[160]^{a}$ \\
\hline \multicolumn{2}{|c|}{$2-\mathrm{CH}_{3}-$} & $109-05-7$ & - & $x$ & $\mathrm{~N}$-Nitroso-2-methylpiperidine & $7247-89-4$ & - & - & $(+)[175,176]^{a}$ \\
\hline \multicolumn{2}{|c|}{$3-\mathrm{CH}_{3}^{-}$} & $626-56-2$ & - & $x$ & N-Nitroso-3-methylpiperidine & $13603-07-1$ & - & - & $(+)[177]^{a}$ \\
\hline \multicolumn{2}{|c|}{$2-\mathrm{COOH}$} & $535-75-1$ & $x$ & - & N-Nitroso-2-piperidinecarboxylic acid & $4515-18-8$ & $x$ & - & $(-)[172]^{a}$ \\
\hline \multirow{2}{*}{\multicolumn{2}{|c|}{$\begin{array}{l}3-\mathrm{COOH} \\
4-\mathrm{COOH}\end{array}$}} & & - & - & N-Nitroso-3-piperidinecarboxylic acid & $65445-62-7$ & $x$ & - & \\
\hline & & & 一 & - & N-Nitroso-4-piperidinecarboxylic acid & 6238-69-3 & $x$ & - & $(-) 173]^{a}$ \\
\hline 2,3-diC & & 23513-39-5 & - & $x$ & $\mathrm{~N}$-Nitroso-2,3-dimethylpiperidine & & - & - & \\
\hline 2,4-diC & & $6287-19-0$ & - & $x$ & N-Nitroso-2,4-dimethylpiperidine & & - & - & \\
\hline 2,6-diC & & $504-03-0$ & - & $x$ & $\mathrm{~N}$-Nitroso-2,6-dimethylpiperidine & $17721-95-8$ & - & - & $(-)[180]^{a}$ \\
\hline $2-\mathrm{C}_{2} \mathrm{H}_{5}-$ & & $1484-80-6$ & - & $x$ & N-Nitroso-2-ethylpiperidine & & - & - & \\
\hline $2-\left(\mathrm{CH}_{3}\right)$ & & & - & $x$ & N-Nitroso-2-isopropylpiperidine & & - & - & \\
\hline 4-keto- & & & - & $x$ & $\mathrm{~N}$-Nitroso-4-piperidone & $55556-91-7$ & - & - & $(+)[163]^{a}$ \\
\hline Pyridine & 1,2,3,6-tetrahydro- (VII) & $694-05-3$ & - & $x$ & N-Nitroso-1,2,3,6-tetrahydropyridine & $55556-92-8$ & - & - & $(+)[168]^{a}$ \\
\hline
\end{tabular}

${ }^{a}$ Bioassay results in laboratory animals are summarized in Preussmann and Stewart (57). (+) indicates tumor induction, (-) indicates negative response. Number in [] is catalog number in (57).

${ }^{b}$ Compounds displayed in bold are listed as toxicants in Table 1 of the main part of our report.

3. Wynder, E.L. and G.F Wright: A study of tobacco carcinogenesis. I. The primary fractions; Cancer 10 (1957) 255-271; Wynder, E.L., G.F Wright, and J. Lam: A study of tobacco carcinogenesis. V. The role of pyrolysis; Cancer 11 (1958) 1140-1148.

4. Lyons, M.J.: Presence of 1,2,3,4-dibenzopyrene in cigarette smoke; Nature 182 (1958) 178.

5. Van Duuren, B.L.: The polynuclear aromatic hydrocarbons in cigarette-smoke condensate. II; J. Natl. Cancer Inst. 21 (1958) 623-630.

6. Rodgman, A. and L.C. Cook: The analysis of cigarette smoke condensate. XIV. Polycyclic aromatic hydrocarbons; RDR, 1960, No. 20, May 26, see www.rirtdocs.com. 501008592 . 8660.

7. Pyriki, C.: Polycyclische und aliphatische Kohlenwasserstoffe des Tabakrauches [Polycyclic and aliphatic hydrocarbons of tobacco smoke]; Die Nahrung 7 (1963) 439-448.

8. Lavit-Lamy, D. and N.P. Buu-Hoï: The true nature of "dibenzo[a,l]pyrene" and its known derivatives; Chem. Comm. 4 (1966) 92-94.

9. Snook, M.E., R.F. Severson, R.F. Arrendale, H.C. Higman, and O.T. Chortyk: The identification of high molecular weight polynuclear aromatic hydrocarbons in a biologically active fraction of cigarette smoke condensate; Beitr. Tabakforsch. Int. 9 (1977) 79-101.

10. Hoffmann, D. and E.L. Wynder: Selective reduction of the tumorigenicity of tobacco smoke. Experimental approaches; in: Toward a less harmful cigarette, edited by E.L. Wynder and D. Hoffmann, Natl. Cancer Inst. Monograph 28 (1968) 151-172, see p. 155, Table 2, Footnote $\S$.

11. IARC: Dibenzo[a,e]fluoranthene; in: Evaluation of the carcinogenic risk of chemicals to humans: Polynuclear aromatic hydrocarbons. Part 1, Chemical, environmental and experimental data; IARC, Lyon, France, IARC Monograph 32 (1986) 321-325.

12. Hecht, S.S.: Tobacco smoke carcinogens and lung cancer; J. Natl. Cancer Inst. 91 (1999) 1194-1210.

13. Snook, M.E., R.F. Severson, R.F. Arrendale, H.C. Higman, and O.T. Chortyk: Multi-alkylated polynuclear aromatic hydrocarbons of tobacco smoke: Separation and identification; Beitr. Tabakforsch. Int. 9 (1978) 222-247.

14. Snook, M.E., R.F. Severson, H.C. Higman, R.F. Arrendale, and O.T. Chortyk: Polynuclear aromatic hydrocarbons of tobacco smoke: Isolation and identification; Beitr. Tabakforsch. 8 (1976) 250-272.

15. Snook, M.E., P.J. Fortson, and O.T. Chortyk: Isolation and 
identification of aza-arenes of tobacco smoke; Beitr. Tabakforsch. Internat. 11 (1981) 67-78.

16. Sasaki, T.A. and S.C. Moldoveanu: Determination of dibenzacridines in the particulate phase of cigarette smoke; Beitr. Tabakforsch. Int. 19 (2000) 25-31.

17. Heckman, R.A. and F.W. Best: An investigation of the lipophilic bases of cigarette smoke condensate; Tob. Sci. 25 (1981) 33-39.

18. Newell, M.P., R.A. Heckman, R.F. Moates, C.R. Green, F.W Best, and J.N. Schumacher: Isolation and identification of new components of the ether-soluble portion of cigarette smoke condensate; Tob. Sci. 22 (1978) 6-11.

19. Schumacher, J.N., C.R. Green, F.W. Best, and M.P. Newell: Smoke composition. An extensive investigation of the watersoluble portion of cigarette smoke; J. Agr. Food Chem. 25 (1977) 310-320.

20. Hoffmann, D. and S.S. Hecht: Advances in tobacco carcinogenesis; in: Chemical carcinogenesis and mutagenesis. I, edited by C.S. Cooper and P.L. Grover, Springer-Verlag, London, UK, 1990, Chapter 3, pp. 63-102.

21. Occupational Safety and Health Administration: Indoor air quality; Fed. Reg. 59 (No. 65) (1994) 15968-16039.

22. Hoffmann, D. and I. Hoffmann: [Chemical studies on tobacco smoke. C]. The changing cigarette: 1950-1995; J. Toxicol. Environ. Hlth. 50 (1997) 307-364.

23. Rothwell, K. and J.K. Whitehead: A method for the concentration of basic polycyclic heterocyclic compounds and the separation of polycyclic aromatic hydrocarbons from cigarette smoke condensate; Chem. and Ind. (London) (1969) 1628-1630.

24. Dipple, A., R.C. Moschel, and C.A.H. Bigger: Polynuclear hydrocarbons; Chapter 2, in: Chemical carcinogens, Second edition, edited by C.E. Searle, Am. Chem. Soc. Monograph 182, American Chemical Society, Washington, DC, 1984, 41-163.

25. Pietzsch, A.: Zum Nachweis von cancerogenen Kohlenwasserstoffen im Tabakrauch [On the detection of carcinogenic hydrocarbons in tobacco smoke]; Naturwissenschaften 45 (1958) 445; Pharmazie (Berlin) 14 (1959) 466-473.

26. Hartwell, J.L.: Survey of compounds which have been tested for carcinogenic activity; USPHS Publ. No. 149 (1947) Washington, DC; Survey of compounds which have been tested for carcinogenic activity; USPHS Publ. No. 149, $2^{\text {nd }}$ Edition (1951) Washington, DC.

27. Shubik, P. and J.L. Hartwell: Survey of compounds which have been tested for carcinogenic activity, Suppl. 1; USPHS Publ. No. 149 (1957) Washington, DC; Suppl. 2; USPHS Publ. No. 149 (1969) Washington, DC.

28. Lee, M.L., M. Novotny, and K.D. Bartle: Gas chromatography/mass spectrometric and nuclear magnetic resonance spectrometric studies of carcinogenic polynuclear aromatic hydrocarbons in tobacco and marijuana smoke condensate; Anal. Chem. 48 (1976) 405-416.

29. Bonnet, J. and S. Neukomm: Sur la composition chimique de la fumée du tabac. I. Analyse de la fraction neutre [The chemical composition of tobacco smoke. I. Analysis of the neutral fraction]; Helv. Chim. Acta 39 (1956) 1724-1733.

30. Brunnemann, K.D. and D. Hoffmann: Analysis of polynuclear aromatic hydrocarbons in the respiratory environment; Carc. Comp. Serv. 1 (1976) 283-297.

31. Lyons, M.J. and H. Johnston: Chemical analysis of the neutral fraction of cigarette smoke tar; Brit. J. Cancer 11 (1957) 554-562.

32. Rothwell, K. and J.K. Whitehead: A method for the isolation of polycyclic aromatic hydrocarbons from complex hydrocarbon mixtures; Chem. and Ind. (London) (1967) 784-786.

33. Grimmer, G., K.W. Naujack, and G. Dettbarn: Gas chromatographic determination of polycyclic aromatic hydrocarbons, aza-arenes, aromatic amines in the particle and vapour phase of mainstream and sidestream smoke of cigarettes; Toxicol Lett. 35 (1987) 117-124.
34. Klimisch, H.J. and S. Beiss: Separation of $N$-heterocyclic aromatic hydrocarbons from polycyclic aromatic hydrocarbons. Separation by ion-exchange chromatography and fractionation of cigarette smoke condensate; J. Chromatog. 128 (1976) 117-124.

35. Pailer, M., K. Völlmin, C. Karninen, and H. Kuhn: Über das Vorkommen von primären und sekundären Aminen im Zigarettenrauch [On the occurrence of primary and secondary amines in cigarette smoke]; Fachl. Mitt. Österr. Tabakregie 10 (1969) 165-168.

36. Brunnemann, K.D., H.C. Lee, and D. Hoffmann: Chemical studies on tobacco smoke. XLVII. On the quantitative analysis of catechols and their reduction; Anal. Lett. 9 (1976) 939-955.

37. IARC: Chemistry and analysis of tobacco smoke; in: Evaluation of the carcinogenic risk of chemicals to humans: Tobacco smoking; IARC, Lyon, France, IARC Monograph 38 (1986) 86-87, Table 19

38. Rodgman, A. and L.C. Cook: The composition of cigarette smoke. XIII. Eugenol and isoeugenol from Turkish tobacco smoke; Tob. Sci. 8 (1964) 161-162.

39. National Technical Information Service: Carcinogenesis studies of eugenol in F344/N rats and B6C3F1 mice (feed studies); TR-223 (NTIS \# PB84-186402) (December 1983).

40. National Technical Information Service: Developmental toxicity evaluation for isoeugenol administered by gavage to Sprague-Dawley $(\mathrm{CD} \AA)$ rats on gestational days 6 through 19; TER97006 (NTIS\# PB2000-105138) (August 1999).

41. Schmeltz, I., J. Tosk, G. Jacobs, and D. Hoffmann: Redox potential and quinone content of cigarette smoke; Anal. Chem. 49 (1977) 1924-1929.

42. Takizawa, N.: Carcinogenic action of certain quinones; Gann 34 (1940) 1; Gann 34 (1940) 158; Gann 34 (1940) 327; Proc. Imp. Acad. Japan 16 (1941) 309.

43. Tiedemann, H.: Does $p$-benzoquinone have a carcinogenic effect on local application? Z. Naturforsch. 8B (1953) 49-50.

44. Benner, J.F., C.K. Keene, and T.W. Holt: Smoke analysis, condensate preparation and condensate fractionation; Proc. Univ. Kentucky Tob. Hlth. Workshop, 1973 Conf. Rpt., Lexington KY. 4 (1973) 408-420.

45. Snook, M.E., P.J. Fortson, and O.T. Chortyk: Application of gel chromatography to characterize more completely the phenols of cigarette smoke; Tob. Sci. 24 (1980) 30-36.

46. Kobashi, Y. and S. Sakaguchi: Free sugars in cigarette smoke; Tob. Sci. 3 (1959) 161-163; Sci. Papers, Cent. Res. Inst., Japan Monopoly Corp. 102 (1960) 16-19.

47. Takizawa, N.: The carcinogenicity of simple sugars; Gann 32 (1938) 236-237; Gann 33 (1939) 193-195.

48. Takizawa, N.: The carcinogenicity of simple sugars; Gann 34 (1940) $1-5$.

49. Holzer, G., J. Oró, and W. Bertsch: Gas chromatographicmass spectrometric evaluation of exhaled tobacco smoke; J. Chromatog. 126 (1976) 771-785.

50. IARC: Some inorganic substances, chlorinated hydrocarbons, $N$-nitrosamines, and natural products; in: Evaluation of the carcinogenic risk of chemicals to humans; IARC, Lyon, France, IARC Monograph 1 (1972) 61.

51. Grob, K, and A. Völlmin: GC-MS analysis of the "semivolatiles" of cigarette smoke; J. Chromat. Sci. 8 (1970) 218-220.

52. IARC: Some naturally occurring substances; in: Evaluation of the carcinogenic risk of chemicals to humans; IARC, Lyon, France, IARC Monograph 10 (1976).

53. IARC: Some fumigants, the herbicides 2,4-D and 2,4,5-T, chlorinated dibenzodioxins and miscellaneous industrial chemicals; in: Evaluation of the carcinogenic risk of chemicals to humans, IARC, Lyon, France, IARC Monograph 15 (1977) 265.

54. Kosak, A.I.: The composition of tobacco smoke; Experientia 10 (1954) 69-71. 
55. Rodgman, A.: Studies of polycyclic aromatic hydrocarbons in cigarette mainstream smoke: Identification, tobacco precursors, control of levels: A review; Beitr. Tabakforsch. Int. 19 (2001) 361-379.

56. Castonguay, A., D. Lin, G.D. Stoner, K. Furuya, S.S. Hecht, H.A.J. Schut, and J.E. Klaunig: Comparative carcinogenicity in $\mathrm{A} / \mathrm{J}$ mice and metabolism by cultured mouse peripheral lung of $N$-nitrosonornicotine, 4-(methylnitrosamino)-1-(3pyridyl)-1-butanone and their analogues; Cancer Res. 43 (1983) 1223-1229.

57. Preussmann, R. and B.W. Stewart: $N$-Nitroso carcinogens; Chapter 12, in: Chemical carcinogens. Second edition, edited by C.E. Searle, American Chemical Society Monograph 182, American Chemical Society, Washington, DC, 1984, 643828.
58. Hoffmann, D. and S.S. Hecht: Advances in tobacco carcinogenesis; in: Chemical carcinogenesis and mutagenesis. I, edited by C.S. Cooper and P.L. Grover, Springer-Verlag, London, UK, 1990, Chapter 3, pp. 63-102; Hoffmann, D., I. Hoffmann, and K. El-Bayoumy: The less harmful cigarette: A controversial issue. A tribute to Ernst L. Wynder; Chem. Res. Toxicol. 14 (2001) 767-790; Hoffmann, D. and I. Hoffmann: [Chemical studies on tobacco smoke. C.] The changing cigarette: 1950-1995; J. Toxicol. Environ. Hlth. 50 (1997) 307-364; The changing cigarette: Chemical studies and bioassays; in: Risks associated with smoking cigarettes with low machine-measured yields of tar and nicotine, NCI Smoking and tobacco control, Monograph 13, edited by D.M. Burns and N.L. Benowitz, Bethesda, MD, Chapter 5, 2001, 159-191. 